\title{
Aggregation and Gelation in Random Networks
}

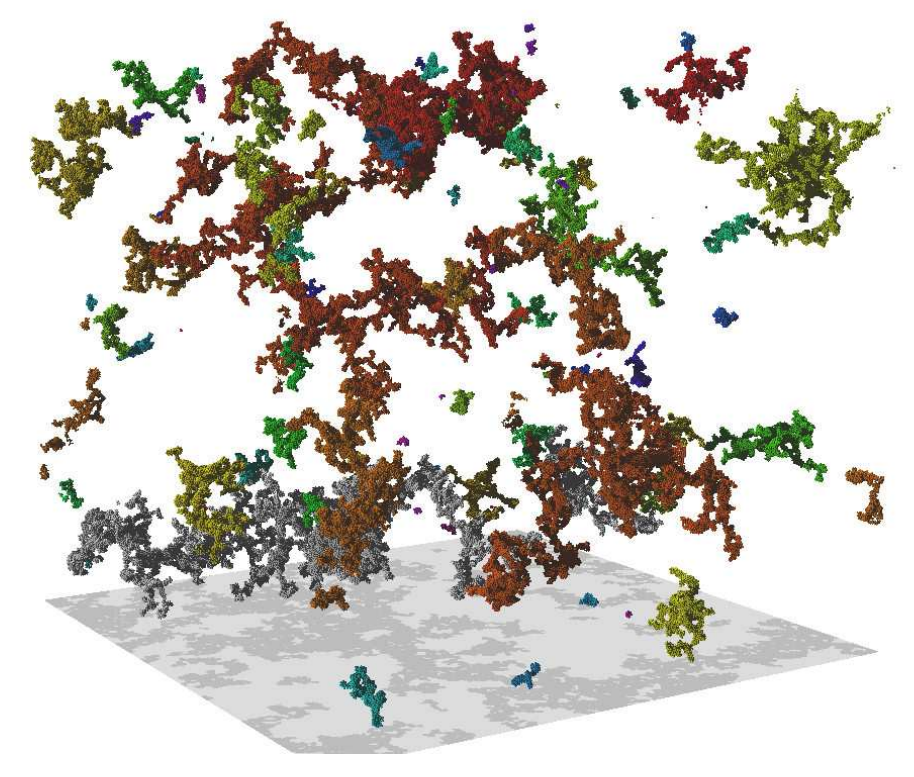

\author{
Dissertation \\ zur Erlangung des Doktorgrades \\ der Mathematisch-Naturwissenschaftlichen Fakultäten \\ der Georg-August-Universität Göttingen
}

vorgelegt von

Stephan Ulrich

aus Karlsruhe

Göttingen 2010 
D7

Referentin: Prof. Dr. Annette Zippelius Koreferent: Prof. Dr. Tim Salditt

Tag der mündlichen Prüfung: 3. 3. 2010 
"Believe those who are seeking the truth; doubt those who find it."

— André Gide (1869-1951) 



\section{Acknowledgments}

First of all, I'm glad for this opportunity to thank Prof. Annette Zippelius for her committed mentoring and encouragement, the excellent scientific support in virtually any problem or difficulty, and a great balance between supervision and freedom. Many times, I was astonished by her incredible physical intuition and her ability to immediately "see" the rough solution of problems, which took me weeks to calculate.

I also would like to thank Prof. Tim Salditt and Anja Glišović for a delightful collaboration and excellent ideas and discussions on the structure determination of spider silk, and also for providing the experimental X-ray images. I thank Martin Meling for a cooperation on the construction of the parallel and antiparallel raw structures of the $\beta$-sheets.

I'm very grateful to Timo Aspelmeier, who let me use the molecular dynamics code, developed by him to simulate granular materials. I also acknowledge a very fruitful collaboration with Prof. Stephan Herminghaus, Klaus Röller and Axel Fingerle, thank you for various enlightening discussions and providing raw data of the thin film model.

Furthermore, I like to thank my friends at the Institut für Theoreische Physik for valuable discussions, enjoyable tea and coffee times and get-togethers (in the order of appearance): Alice von der Heydt*, Till Kranz*, Andrea Fiege, Martin Kiemes*, Richard Vink, Timo Fisher and Johnathan Fish*. The star ${ }^{*}$ denotes especial acknowledgment for proof-reading this thesis.

A big thank you also to the secretaries, Katrin Glormann, Gabriele Schubert, Bärbel Lütge-Hampe and Gisela Mesecke for handling all administrative issues quickly and professionally. Part of this work was supported by the DFG through SFB 602 and Grant No. Zi 209/7 (S.U,A.Z), which is gratefully acknowledged. Last but certainly not least, I thank my family for their emotional support. 



\section{Contents}

1 Introduction 1

2 Highly Cross-Linked Brownian Particles 3

2.1 Introduction . . . . . . . . . . . . . . . . 3

2.2 Model: Randomly Cross-Linked Particles . . . . . . . . . . . . . 4

2.3 Disorder Average . . . . . . . . . . . . . . . . . . . 6

2.4 Replica Calculation of the Free Energy . . . . . . . . . . . . . 7

2.4.1 Disorder-Averaged Free Energy and Introduction of Replicas 7

2.4.2 Introduction of the Replicated Density Fields . . . . . . . . . 9

2.4.3 Free Energy of the Replicated Density Field . . . . . . . . . . 11

2.4.4 Introduction of a Field Theory and Decoupling . . . . . . . . 12

2.4.5 Universality and Connection to Other Models . . . . . . . . . 13

2.5 Goldstone Fluctuations in General . . . . . . . . . . . . . . . . . . 14

2.6 Ansatz for the Order Parameter . . . . . . . . . . . . . . . 15

2.6.1 Spontaneously Broken Translational Invariance . . . . . . . . 17

2.6.2 Goldstone Fluctuations and Ansatz for $\Omega$. . . . . . . . 18

2.6.3 Physical Shear Deformations . . . . . . . . . . . . 20

2.7 Replica Free Energy with Shear Deformations . . . . . . . . . . . . . 21

2.8 Returning to the Disorder-Averaged Free Energy . . . . . . . . . . 23

2.9 Results . . . . . . . . . . . . . . . . . . . . . 24

2.9.1 Average Cross-Link Density and Higher Moments . . . . . . 24

2.9 .2 Gel Fraction . . . . . . . . . . . . . . . . 25

2.9 .3 Shear Modulus . . . . . . . . . . . . . . . . 27

2.10 Conclusions . . . . . . . . . . . . . . . . . . . . 29

3 Cross-Linked Directed Polymers 31

3.1 Introduction . . . . . . . . . . . . . . . . . . . 31

3.2 Properties of a Single Chain . . . . . . . . . . . . . . . . 33

3.2.1 Radius of Gyration of an Uncrosslinked Chain . . . . . . . 33

3.2 .2 Properties of a Polymer Clamped in Space . . . . . . . . . . 34

3.3 Full Model: Cross-Linked Directed Polymers . . . . . . . . . . . . 36

3.3 .1 Interactions . . . . . . . . . . . . . . . 36

3.3.2 Disorder Average . . . . . . . . . . . . . . . . . . . . 39

3.4 Replica Calculation of the Free Energy . . . . . . . . . . . . . . . . 40

3.4.1 Disorder-Averaged Free Energy: First Steps . . . . . . . . . . 40

3.4.2 Introduction of the Replicated Density Field . . . . . . . . . 41

3.4.3 Introduction of a Field Theory and Decoupling . . . . . . . . 43

3.4 .4 Saddle Point Equation . . . . . . . . . . . . . . . . . . . 44

3.5 Ansatz for the Order Parameter . . . . . . . . . . . . . . . . 45

3.6 The Saddle-Point Equation with Ansatz for $\Omega \ldots \ldots$. . . . . . . 47 
3.6.1 Expansion to second order in $Q \ldots \ldots \ldots \ldots$

3.6 .2 Sol-Gel Transition . . . . . . . . . . . . . . . . . . . 49

3.7 The Equation for the Localization Length . . . . . . . . . . . . . 49

3.7.1 Normalization of Length Scales . . . . . . . . . . . . . . . 49

3.7.2 Result for the Distribution of Localization Lengths . . . . . . 50

3.8 Conclusions . . . . . . . . . . . . . . . . . . 55

4 Structure of Spider Silk Modeled as a Random Network $\quad 57$

4.1 Introduction . . . . . . . . . . . . . . . . . . 57

4.2 Model . . . . . . . . . . . . . . . . . . . . 60

4.2 .1 Unit Cell . . . . . . . . . . . . . . . . . 60

4.2 .2 Crystallite . . . . . . . . . . . . . . . . . . . 60

4.2 .3 Ensemble of Crystallites . . . . . . . . . . . . . . 63

4.2 .4 Continuous Background . . . . . . . . . . . . . . 64

4.3 Scattering Function . . . . . . . . . . . . . . . . . 65

4.3.1 Incoherent Part $G_{1}(\mathbf{q}) \ldots \ldots \ldots \ldots 6$

4.3 .2 Coherent Part $G_{2}(\mathbf{q}) \ldots \ldots \ldots \ldots \ldots 67$

4.3.3 Scattering Amplitude of a Single Crystallite . . . . . . . . . 68

4.4 Atomic Configuration of the Unit Cell . . . . . . . . . . . . . . . 69

4.4.1 Unshifted Unit Cells . . . . . . . . . . . . . . . . . . . 69

4.4.2 Possible Shifts inside the Unit Cell . . . . . . . . . . . . 72

4.4.3 Variations between Crystallites . . . . . . . . . . . . 72

4.5 Results . . . . . . . . . . . . . . . . . 73

4.5.1 Experimental Scattering Function . . . . . . . . . . 73

4.5.2 Scattering Function from the Model . . . . . . . . . . . . 74

4.6 Conclusions . . . . . . . . . . . . . . . . . . . . 79

5 Aggregation and Gelation in Wet Granular Materials $\quad 81$

5.1 Introduction . . . . . . . . . . . . . . . . . . . . . 81

5.2 Models . . . . . . . . . . . . . . . . . . . . . . . . 83

5.3 Cooling Dynamics . . . . . . . . . . . . . . . . 86

5.3 .1 Early Stage of Cooling . . . . . . . . . . . . . . 87

5.3 .2 Late Stage of Cooling . . . . . . . . . . . . . . . . 90

5.3 .3 Velocity Distribution . . . . . . . . . . . . . . . . 95

5.3.4 Partitioning of the Energy into Translational, Rotational and

Internal Degrees of Freedom . . . . . . . . . . . . . 98

5.4 Aggregation . . . . . . . . . . . . . . . . . . . 101

5.4 .1 Self-Similar Growth . . . . . . . . . . . . . . . . 103

5.4.1.1 Fractal Dimension of the Aggregates . . . . . . . . 103

5.4.1.2 Cluster Mass Distribution . . . . . . . . . . . 105

5.4.1.3 Number of Clusters and Mean Cluster Mass . . . . 106

5.4 .2 Properties of the Asymptotic Cluster . . . . . . . . . . . 107

5.4.2.1 Fractal Dimension from Radius of Gyration . . . . . 108

5.4.2.2 Fractal Dimension from Box Counting Algorithm . 110 
5.4.2.3 Pair Correlation Function and Correlation Dimension 114

5.4.2.4 Coordination Number . . . . . . . . . . . . . 118

5.4.2.5 Inertia Tensor and Spatial Extension . . . . . . . 122

5.5 Conclusions . . . . . . . . . . . . . . . . . . . 123

A Properties of $\perp$ - and $\|$-vectors $\quad 125$

B Calculations for the Randomly Cross-Linked Particle Model $\quad 127$

B.1 Calculation of the Replica Free Energy . . . . . . . . . . . . . 128

B.1.1 Emergence of the Replica Free Energy . . . . . . . . . . . . 128

B.1.2 Introduction of the Replicated Density Field . . . . . . . . . 129

B.2 Hubbard-Stratonovich Transformation . . . . . . . . . . . . . . 130

B.2.1 Applying the Hubbard-Stratonovich Transformation . . . . . 130

B.2.2 Meaning of the Order Parameter $\Omega(\hat{q}) \ldots \ldots \ldots \ldots$

B.3 Expansion of the replica free energy to 3rd Order in $\Omega$. . . . . . 134

B.4 Fluctuations around the Saddle-Point Solution . . . . . . . . . . . 135

B.5 Replica Free Energy with Shear Deformations . . . . . . . . . . . . . 136

B.5.1 Preparing the Replica Free Energy for the Ansatz . . . . . . 136

B.5.2 Expansion of the one-particle partition function $\mathfrak{z} \ldots \ldots . .137$

B.5.3 Insertion of the Order Parameter in $\mathfrak{z} \ldots \ldots \ldots . . . . .137$

B.5.4 Insertion of the Order Parameter in $f_{n+1}\{\Omega\} \ldots \ldots . \ldots 145$

B.5.5 Recomposing the Replica Free Energy . . . . . . . . . . . . 146

B.5.6 Analysis of the quantity $\Xi_{r, a^{2}} \ldots \ldots \ldots \ldots$. . . . . . . . . . . . . . 149

B.6 Auxiliary Calculations for the Results Section . . . . . . . . . . 150

B.6.1 Distribution of the Number of Cross-Links . . . . . . . . . 150

B.6.2 Shear modulus . . . . . . . . . . . . . . . . . . . 152

B.6.3 Square of the expansion for $(1-Q) \ldots \ldots \ldots \ldots$

B.7 The Order Parameter in the One Replica Sector . . . . . . . . . . 154

C Calculations for the Directed Polymer Model 157

C.1 Calculations for a Single Chain . . . . . . . . . . . . . . . 157

C.1.1 The Uncrosslinked Chain . . . . . . . . . . . . . . 157

C.1.2 The Polymer Clamped in Space . . . . . . . . . . . . . . . . . 159

C.2 Examples of Single Chain Interactions . . . . . . . . . . . . 160

C.3 Calculations for the Disorder-Averaged Free Energy . . . . . . . . 162

C.3.1 Introduction of Replicas . . . . . . . . . . . . . . . 162

C.3.2 Introduction of the Replicated Density Field . . . . . . . . 163

C.3.3 The Hubbard-Stratonovich Transformation . . . . . . . . . . 164

C.4 The Saddle-Point Equation with Ansatz . . . . . . . . . . . . . . 167

C.4.1 Expansion in $Q$ to Infinite Order . . . . . . . . . . 167

C.4.2 Expansion to Second Order in $Q$. . . . . . . . . . . 169

C.5 Obtaining the Equation for the Localization Length . . . . . . . 173

C.5.1 Normalization of Length Scales . . . . . . . . . . . . . . . 173

C.5.2 Laplace-Transformation of the Saddle Point Equation . . . . 175 
C.6 Calculation of a Correlator . . . . . . . . . . . . . . . . . 177

C.7 The Average Cross-Link Density . . . . . . . . . . . . . . . . 180

D Expressions for the Wet Granulates 183

D.1 Infinite Sums similar to the Exponential Function . . . . . . . . . . 183

D.2 Inverting the Equation $x^{-2} \mathrm{e}^{x}=t \ldots \ldots \ldots \ldots \ldots$

D.3 Computation of the radial distribution function $g(r) \ldots \ldots$

E Further Results from the Spider Silk Model 187

E.1 Effect of the Continuous Background . . . . . . . . . . . . . 187

E.2 Relevance of the Coherent Part of the Scattering Function . . . . . . 187

E.3 Simplification of the Scattering Amplitude $A(\mathbf{q}) \ldots \ldots . . . .188$

$\begin{array}{lr}\text { F Used Symbols and Notation } & 191\end{array}$

F.1 Notation . . . . . . . . . . . . . . . . . . . . . . . . . . . 191

F.2 Symbols for the Randomly Cross-Linked Particle Model . . . . . . . 192

F.3 Symbols for the Cross-Linked Directed Polymers . . . . . . . . . . . 194

F.4 Symbols for the Spider Silk Model . . . . . . . . . . . . . . . . . 197

F.5 Symbols for the Wet Granular System . . . . . . . . . . . . . 199

$\begin{array}{ll}\text { Bibliography } & 201\end{array}$

$\begin{array}{lr}\text { Index } & 211\end{array}$

$\begin{array}{lr}\text { Curriculum Vitae } & 215\end{array}$ 
CHAPTER 1

\section{Introduction}

Let us consider a large system, made up of many identical interconnected building blocks, so that close-by building blocks are preferably connected. Then, one may raise a number of questions, for example, under which conditions it is possible to go from one side of the system all the way to the other side, only using the connections (or equivalently that a "percolating" or "macroscopic" cluster is present). Or one may ask, what the structure of the clusters is, which the connections give rise to, for example how large the cluster are and whether or not they have a fractal structure.

These types of problems are known under the term percolation theory and despite their seemingly abstract and simple concepts, they are highly relevant for many problems in physics and for a wide range of applications in every day's life. An example would be the spreading of forest fires: will a fire - starting at one tree eventually span the whole forest, or will it be extinguished quickly only burning down a few trees? For this case, we imagine that our "building blocks" are the trees in the forest and we define them as connected if they are close enough so that a burning tree would ignite the other one. Then, the cluster sizes of this system will provide indications what the typical extent of the fire will be, and the presence of a macroscopic cluster gives information whether or not the fire may span the whole system. Further examples include filtering of fluids or gases through porous materials, e.g. movement of petroleum through fractured rock, the flow of electrical current through a random resistor network, or the spreading of diseases in a social network.

Another physically important example, treated in this thesis, is polymerization, where chemical bonds connect atoms or molecules and can thereby create large networks of macromolecules. To achieve this, one may start with a system of polymer chains (typically chains of connected carbon atoms) in a solvent and add a certain amount of cross-linkers (typically sulfur). Thereupon, one observes that the cross-links interconnect the chains, whereby larger and larger aggregates are formed. When the concentration of the cross-links exceeds a critical value, one can find an aggregate that spans the whole system. In this process the system undergoes a transition from a liquid (sol) to an amorphous solid (gel) phase, the so called "gelation transition". Materials created in this way (like for example rubber) have interesting properties in terms of flexibility and toughness. It is important to note they have no lattice structure, in comparison to metals; instead they are amorphous because the aggregation mechanism always involves some randomness, and consequently they are translationally and rotationally invariant on a macroscopic scale. 
The formation, structure and properties of aggregates created by random connections, and in particular the above-mentioned gelation transition are the topic of this thesis. In chapter 2 we will discuss cross-linked Brownian particles and particularly their elastic properties. When the cross-link density is large enough, such that a macroscopic cluster is present, the system exhibits a finite resistance to shear deformations. This mechanical resistance is quantified by the shear modulus, the focus of this chapter. We extend previous results, which were restricted to systems close to the gelation transition, to highly cross-linked gels.

In chapter 3 we investigate cross-linked directed polymers, where the polymer chains are aligned along a preferential direction. This anisotropic system is a simple model for describing polymers, highly stretched along one direction, or alternatively polymers in a nematic field. As cross-links are added to this system, we observe a gelation transition and investigate properties of the system close to that transition. Focussing on the distribution of localization lengths, we describe the extent of fluctuations of the chains about their mean positions. As we will see, the anisotropy of the system gives rise to a dependence of these structural properties on the distance from the boundaries in the preferred direction.

In chapter 4 we determine the microscopic structure of spider-silk, where small crystallites are interconnected by a network of chains. This composition of the material gives rise to outstanding mechanical properties, in particular the toughness and extensibility. We develop a statistical model for the crystallites; from that, the scattering function can be computed and be compared to experimental results, enabling us to determine structural, as well as statistical parameters of the system. We also investigate the role of coherent scattering from different crystallites and the importance of a background scattering density between crystallites.

And in chapter 5 we study the aggregation of wet granular particles. Here, the particles conjoin and develop clusters, because they are covered by a thin liquid film and may form "liquid bridges" between each other if the films touch each other and merge. These bonds induce an attractive and hysteretic interaction such that each bond rupture dissipates a fixed amount of energy. The system shows an interesting cooling behavior different from dry granulates, and a transition from fast cooling to a clustering state as soon as the particles' kinetic energy is not sufficient to break the bonds anymore. As time proceeds, the clusters form larger and larger aggregates, which grow in a self-similar fashion. For all finite densities a percolation transition is observed, with a macroscopic cluster that is fractal on small length scales and compact on large length scales.

A number of technical points have been deferred to the appendices. In particular, the symbols and variables used in each chapter and a short remark on the notation can be found in Appendix F. 
Chapter 2

\title{
Highly Cross-Linked Brownian Particles
}

\author{
Contents

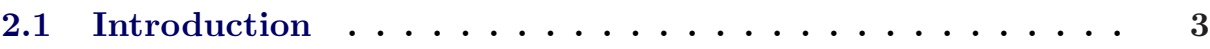 \\ 2.2 Model: Randomly Cross-Linked Particles . . . . . . . . . 4 \\ 2.3 Disorder Average $\ldots \ldots \ldots \ldots \ldots \ldots \ldots$ \\ 2.4 Replica Calculation of the Free Energy . . . . . . . 7 \\ 2.4.1 Disorder-Averaged Free Energy and Introduction of Replicas . 7 \\ 2.4.2 Introduction of the Replicated Density Fields . . . . . . . . 9 \\ 2.4.3 Free Energy of the Replicated Density Field . . . . . . . . . . 11 \\ 2.4.4 Introduction of a Field Theory and Decoupling . . . . . . . . 12 \\ 2.4.5 Universality and Connection to Other Models . . . . . . . . . 13
}

2.5 Goldstone Fluctuations in General . . . . . . . . . . . . 14

2.6 Ansatz for the Order Parameter . . . . . . . . . 15

2.6.1 Spontaneously Broken Translational Invariance . . . . . . . 17

2.6.2 Goldstone Fluctuations and Ansatz for $\Omega$. . . . . . . . 18

2.6.3 Physical Shear Deformations . . . . . . . . . . . . . . 20

2.7 Replica Free Energy with Shear Deformations . . . . . . . 21

2.8 Returning to the Disorder-Averaged Free Energy . . . . . . 23

2.9 Results . . . . . . . . . . . . . . . . 24

2.9.1 Average Cross-Link Density and Higher Moments . . . . . . 24

2.9 .2 Gel Fraction . . . . . . . . . . . . . . . . . . . 25

2.9 .3 Shear Modulus . . . . . . . . . . . . . . . . . 27

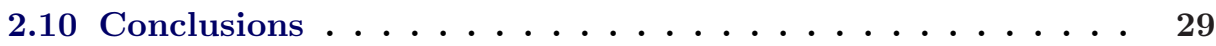

\section{$2.1 \quad$ Introduction}

Randomly cross-linked networks of molecules undergo a transition from a fluid to an amorphous solid state, as the number of cross-links is increased. By adding more and more permanent cross-links to the system, molecular clusters are gradually joined and in this process grow to larger and larger objects. Thereby the 
system undergoes a transition from the sol phase, a complex fluid characterized by molecular clusters of various but finite sizes, to the gel phase, an amorphous solid where the constituents of the system have joined to at least one macroscopic (system spanning) cluster yielding a non-zero resistance to shear deformations. This sol-gel transition or gelation transition has been studied in recent years [Deam \& Edwards, 1976; Ball \& Edwards, 1980; Goldbart et al., 1996; Panyukov \& Rabin, 1996], and the structure as well as the elasticity [Mukhopadhyay et al., 2004; Goldbart et al., 2004] has been investigated. However, almost all theoretical studies have focussed on the near-critical region, where the analysis simplifies due to the existence of a small parameter - the distance to the transition point or, equivalently, the order parameter. In contrast, the highly cross-linked regime has hardly been investigated [Broderix et al., 2002], yet it is particularly interesting in the application of random network models to glasses [Zallen, 1983].

Here we consider a particularly simple network which is built from spherical particles, connected by harmonic springs. This allows us to access the highly crosslinked regime and compute the shear modulus for arbitrary cross-link concentrations. It is found that the shear modulus is independent of all microscopic length scales of the model, such as the spring constant and the length scale that characterizes the localization of particles in the amorphous solid state. Instead, the shear modulus is completely determined by the particle density and the density of cross-links.

We approach the problem using equilibrium thermodynamics. The average over all cross-link configurations - the so called disorder average - is done with the help of the replica theory. Furthermore, to simplify the notation, energies are measured in units of $k_{\mathrm{B}} T$.

The chapter is organized as follows: In Sec. 2.2 the model of randomly crosslinked particles and its interactions are presented and in Sec. 2.3 the disorder average is defined. In Sec. 2.4 we establish a field theory of the presented model and find a suitable order parameter. In Sec. 2.5 we have a short general look at Goldstone fluctuations, which are necessary to establish an Ansatz for the order parameter, done in Sec. 2.6. In Sec. 2.7 we apply this Ansatz to the field theory, and establish the connection of the result to the actual disorder-averaged free energy of the system in Sec. 2.8. The final results are presented in Sec. 2.9, and we will conclude in Sec. 2.10 .

\subsection{Model: Randomly Cross-Linked Particles}

We consider a system of $N$ identical particles at positions $\mathbf{r}_{1}, \ldots, \mathbf{r}_{N}$ in a $D$ dimensional volume $V$. Permanent cross-links connect $M$ randomly chosen pair of particles, such that a particular cross-link realization $\mathcal{C}$ is specified by a list of $M$ pairs of monomers $\mathcal{C}=\left\{\left(i_{1}, j_{1}\right), \ldots,\left(i_{M}, j_{M}\right)\right\}$ (see Fig. 2.1). The cross-links are modeled by harmonic springs, so that the Hamiltonian for the cross-link interaction becomes: 


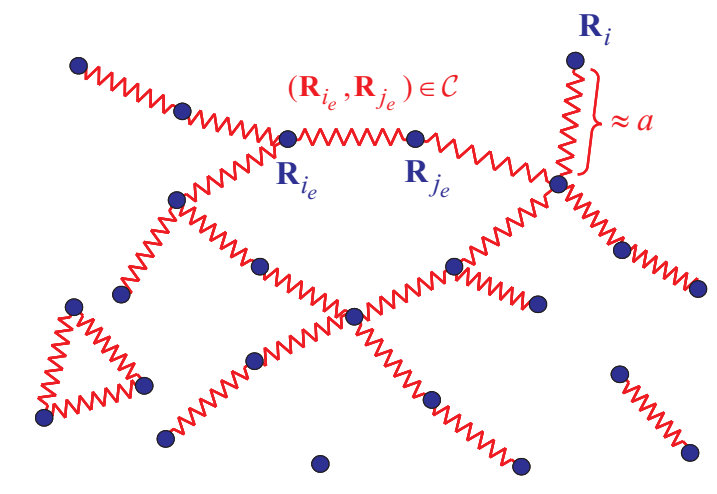

Figure 2.1: Illustration of the randomly cross-linked particle model. Particles $(\bullet)$ are permanently connected via Hookian springs ( $\mathrm{mm}$ ), which have a typical cross-link distance of $a$. Double bonds are also possible, as a pair of particles can in principle be connected twice. The cross-link configuration defines clusters of different sizes, but unconnected particles are possible as well.

$$
H_{\mathrm{Xlink}}=\frac{1}{2 a^{2}} \sum_{e=1}^{M}\left(\mathbf{r}_{i_{e}}-\mathbf{r}_{j_{e}}\right)^{2}
$$

Here, the parameter $a$ can be interpreted as the typical length of a cross-link. One can see this by calculating the typical distance between two cross-linked particles $\mathbf{r}_{1}, \mathbf{r}_{2}$ without further interactions:

$$
\left\langle\left(\mathbf{r}_{1}-\mathbf{r}_{2}\right)^{2}\right\rangle=\frac{\int \mathrm{d} \mathbf{r}_{1} \mathrm{~d} \mathbf{r}_{2}\left(\mathbf{r}_{1}-\mathbf{r}_{2}\right)^{2} \exp \left(-\frac{\left(\mathbf{r}_{1}-\mathbf{r}_{2}\right)^{2}}{2 a^{2}}\right)}{\int \mathrm{d} \mathbf{r}_{1} \mathrm{~d} \mathbf{r}_{2} \exp \left(-\frac{\left(\mathbf{r}_{1}-\mathbf{r}_{2}\right)^{2}}{2 a^{2}}\right)} .
$$

We substitute $\mathbf{r}:=\mathbf{r}_{1}-\mathbf{r}_{2}$ and perform one of the integrals, which cancels in numerator and denominator. Then we are left with:

$$
\left\langle\left(\mathbf{r}_{1}-\mathbf{r}_{2}\right)^{2}\right\rangle=\frac{\int \mathrm{d} \mathbf{r} \mathbf{r}^{2} \exp \left(-\frac{\mathbf{r}^{2}}{2 a^{2}}\right)}{\int \mathrm{d} \mathbf{r} \exp \left(-\frac{\mathbf{r}^{2}}{2 a^{2}}\right)} .
$$

These are two Gaussian integrals which can easily be calculated:

$$
\left\langle\left(\mathbf{r}_{1}-\mathbf{r}_{2}\right)^{2}\right\rangle=\frac{\left(2 \pi a^{2}\right)^{D / 2} \cdot D a^{2}}{\left(2 \pi a^{2}\right)^{D / 2}}=D a^{2}
$$

Furthermore, a repulsive excluded-volume interaction is introduced, which acts between all pairs of particles:

$$
H_{\mathrm{ev}}=\frac{\lambda}{2} \sum_{i, j=1}^{N} U\left(\mathbf{r}_{i}-\mathbf{r}_{j}\right)
$$


This interaction is necessary to prevent the system from collapsing. Its strength $\lambda$ has to be large enough to overcome the attractive interaction produced by the cross-links, and the function $U(\mathbf{x})$ has to fall off fast enough (at the end of Sec. 2.4.3 we will see, what that means specifically). Since $\lambda$ already scales the strength of the interaction we take the freedom to require $\int_{V} U(\mathbf{x}) \mathrm{d} \mathbf{x}=1$.

The overall Hamiltonian is $H_{\mathcal{C}}=H_{\mathcal{C}}\left(\left\{\mathbf{r}_{j}\right\}\right)=H_{\text {Xlink }}+H_{\text {ev }}$. The index $\mathcal{C}$ will remain as a reminder on variables that depend on the quenched disorder $\mathcal{C}$ specifying the configuration of cross-links. All thermodynamic properties, including the elastic ones, can be obtained from the partition function

$$
Z_{\mathcal{C}}=\int \operatorname{Dr}_{1} \cdots \operatorname{Dr}_{N} \mathrm{e}^{-H_{\mathcal{C}}\left(\left\{\mathbf{r}_{j}\right\}\right)}
$$

Here we use the measure $D \mathbf{r}_{j}:=\mathrm{d} \mathbf{r}_{j} / V$ to make sure that $Z_{\mathcal{C}}$ is dimensionless.

This model became known as the randomly (cross-)linked particle (RLP) model, and is a very simple model for amorphous solids. As we see in the illustration 2.1 it can produce highly branched "molecule chains". It is capable of predicting structural and mechanical properties [Broderix et al., 2002; Mao et al., 2007] and has been investigated with and without the use of replica technique. As we will see in Sec. 2.4.5, in the vicinity of the gelation transition, the model falls into the universality class of randomly cross-linked media.

\subsection{Disorder Average}

The average over all cross-link configurations $\mathcal{C}$ is called the disorder average and denoted by $[\cdot]$. The disorder average of a quantity $\mathcal{A}_{\mathcal{C}}$ is calculated by:

$$
\left[\mathcal{A}_{\mathcal{C}}\right]=\sum_{M=0}^{\infty} \sum_{\substack{i_{1}, \ldots, i_{M}, j_{1}, \ldots, j_{M}=1}}^{N} \mathcal{A}_{\mathcal{C}} \cdot P(\mathcal{C})
$$

For the cross-link distribution $P(\mathcal{C})$, we use the Deam-Edwards distribution [Deam \& Edwards, 1976]:

$$
\begin{aligned}
P_{\mathrm{DE}}(\mathcal{C}) & =\frac{1}{\mathcal{Z}_{1}} \frac{1}{M !}\left(\frac{\mu^{2}}{2 N \phi}\right)^{M} Z_{\mathcal{C}} \\
\text { with } \quad \phi & =\frac{a^{D}(2 \pi)^{D / 2}}{V}
\end{aligned}
$$

and with a normalization constant $\mathcal{Z}_{1}$. On first glance it seems to be counterintuitive that the probability for a cross-link configuration $\mathcal{C}$ is proportional to the corresponding partition function $Z_{\mathcal{C}}$, but this characteristic is a very clever trick to make sure only particles close to each other get connected. In this regard, let us have a look at a cross-link configuration $\mathcal{C}$; by writing the partition function (2.6) slightly differently, one can express the probability for this configuration as

$$
P_{\mathrm{DE}}(\mathcal{C}) \propto\left\langle\mathrm{e}^{-\frac{1}{2 a^{2}} \sum_{e=1}^{M}\left(\mathbf{r}_{i_{e}}-\mathbf{r}_{j_{e}}\right)^{2}}\right\rangle_{H_{\mathrm{ev}}},
$$


where $\langle\cdot\rangle_{H_{\mathrm{ev}}}$ is averaging with the statistical weight of the excluded volume interaction. In this form, one can see that configurations for which the distances $\left|\mathbf{r}_{i_{e}}-\mathbf{r}_{j_{e}}\right|$ are small have a high probability, while configurations that would involve some long cross-links have a low probability. For a detailed discussion of the Deam-Edwards distribution, see the work by Goldbart et al. [1996]; Broderix et al. [2002].

The parameter $\mu^{2}$ determines the probability for a cross-link to be formed, and therefore controls the sol-gel transition. As we will see in Sec. 2.9.1, the average cross-link density is given by $[M] / N=\mu^{2} / 2$ and the standard deviation, relative to the mean, vanishes in the thermodynamic limit. Thus, $\mu^{2}=2[M] / N$ is the average coordination number, i.e. the average number of particles to which a certain particle is connected.

\subsection{Replica Calculation of the Free Energy}

\subsubsection{Disorder-Averaged Free Energy and Introduction of Replicas}

From this model, we now would like to calculate the free energy, in order to access mechanical and structural properties. However, we are not interested in a specific cross-link configuration (and we cannot choose the cross-link configuration for $10^{23}$ particles by hand anyway), but only in typical properties of the system. Therefore we have to calculate the disorder-averaged free energy

$$
\left[F_{\mathcal{C}}\right]=-\left[\ln Z_{\mathcal{C}}\right]
$$

In general it is very difficult to perform the disorder average, Eq. (2.7), of a logarithm. A successful way to perform the sum nevertheless is the replica trick: Instead of averaging the logarithm itself, we take advantage of a representation of the logarithm:

$$
-\left[F_{\mathcal{C}}\right]=\left[\ln Z_{\mathcal{C}}\right]=\left[\lim _{n \rightarrow 0} \frac{Z_{\mathcal{C}}^{n}-1}{n}\right]=\lim _{n \rightarrow 0} \frac{\left[Z_{\mathcal{C}}^{n}\right]-1}{n}
$$

and thus we try to calculate

$$
\left[Z_{\mathcal{C}}^{n}\right]=\frac{1}{\mathcal{Z}_{1}} \sum_{M=0}^{\infty} \sum_{\substack{i_{1}, \ldots, i_{M}, j_{1}, \ldots, j_{M}=1}}^{N} \frac{1}{M !}\left(\frac{\mu^{2}}{2 N \phi}\right)^{M} Z_{\mathcal{C}} \cdot Z_{\mathcal{C}}^{n}
$$

At first, let's have a look at the rightmost part of Eq. (2.11). At least for integer $n, Z_{\mathcal{C}} \cdot Z_{\mathcal{C}}^{n}$ can be written as a product $\prod_{\alpha=0}^{n} Z_{\mathcal{C}}$. By doing that, we introduce $n+1$ copies of the system with identical cross-link configuration (one of 
the copies, which we call the zeroth, stems from the Deam-Edwards distribution):

$$
\begin{aligned}
Z_{\mathcal{C}} & Z_{\mathcal{C}}^{n}=\prod_{\alpha=0}^{n} \int\left(\prod_{j=1}^{N} \mathrm{Dr}_{j}^{(\alpha)}\right) \mathrm{e}^{-H_{\mathcal{C}}\left(\left\{\mathbf{r}_{j}^{(\alpha)}\right\}\right)} \\
& =\int\left(\prod_{j=1}^{N} \prod_{\alpha=0}^{n} \mathrm{Dr}_{j}^{(\alpha)}\right) \exp \left(-\frac{1}{2 a^{2}} \sum_{e=1}^{M} \sum_{\alpha=0}^{n}\left(\mathbf{r}_{i_{e}}^{(\alpha)}-\mathbf{r}_{j_{e}}^{(\alpha)}\right)^{2}-\sum_{\alpha=0}^{n} H_{\mathrm{ev}}\left(\left\{\mathbf{r}_{j}^{(\alpha)}\right\}\right)\right) \\
& =\int\left(\prod_{j=1}^{N} \mathrm{D} \hat{r}_{j}\right) \exp \left(-\frac{1}{2 a^{2}} \sum_{e=1}^{M}\left(\hat{r}_{i_{e}}-\hat{r}_{j_{e}}\right)^{2}-H_{\mathrm{ev}}^{(n+1)}\right),
\end{aligned}
$$

with $H_{\mathrm{ev}}^{(n+1)}:=\sum_{\alpha=0}^{n} H_{\mathrm{ev}}\left(\left\{\mathbf{r}_{j}^{(\alpha)}\right\}\right)$. Here we introduced the $n+1$ times replicated (and hence $D(n+1)$-dimensional) vectors

$$
\hat{r}_{j}:=\left(\mathbf{r}_{j}^{(0)}, \ldots, \mathbf{r}_{j}^{(n)}\right) .
$$

This introduction of $n+1$ replicas of the system ${ }^{1}$ is the main characteristic of replica theory and the reason for its name. One should keep in mind that the disorder is the same in all replicas; in particular $M$ and $i_{e}, j_{e}$ do not have replica indices $(\alpha)$. Assuming ergodicity, a possible way to imagine this replicated system is to see the particle conformations $\left\{\mathbf{r}_{j}^{(0)}\right\},\left\{\mathbf{r}_{j}^{(1)}\right\}, \ldots,\left\{\mathbf{r}_{j}^{(n)}\right\}$ as instances of the system taken at different times, with very long time differences in between. In this picture, the particle conformation in the zeroth replica, $\left\{\mathbf{r}_{j}^{(0)}\right\}$, corresponds to the particle conformation during cross-linking.

As we see in Appendix B.1.1, the sum over $M$ in Eq. (2.11) can be performed and $\left[Z_{\mathcal{C}}^{n}\right]$ can be written as:

$$
\begin{aligned}
{\left[Z_{\mathcal{C}}^{n}\right] } & =\frac{\mathcal{Z}_{n+1}}{\mathcal{Z}_{1}} \\
\text { where } \quad \mathcal{Z}_{n+1} & =\int\left(\prod_{j=1}^{N} \mathrm{D} \hat{r}_{j}\right) \exp \left(-N \tilde{f}_{n+1}\left\{\hat{r}_{j}\right\}\right)
\end{aligned}
$$

with the "replica free energy"

$$
\tilde{f}_{n+1}\left\{\hat{r}_{j}\right\}=-\frac{\mu^{2}}{2 \phi N^{2}} \sum_{i, j=1}^{N} \exp \left(-\frac{\left(\hat{r}_{i}-\hat{r}_{j}\right)^{2}}{2 a^{2}}\right)+\frac{\lambda}{2 N} \sum_{\alpha=0}^{n} \sum_{i, j=1}^{N} U\left(\mathbf{r}_{i}^{(\alpha)}-\mathbf{r}_{j}^{(\alpha)}\right),
$$

which incorporates the degrees of freedom of the system, the replicated particle positions $\left\{\hat{r}_{1}, \ldots, \hat{r}_{N}\right\}$. That means, the disorder-averaged $\left[Z_{\mathcal{C}}^{n}\right]$ for a $D$-dimensional system can be expressed as a partition function $\mathcal{Z}_{n+1}$ that involves $D(n+1)$ dimensional vectors but does not involve a disorder average anymore. We will refer to $\mathcal{Z}_{n+1}$ as the "replica partition function".

$\mathcal{Z}_{1}$ was originally introduced as a mere normalization constant in Eq. (2.8); knowing that $\lim _{n \rightarrow 0}\left[Z_{\mathcal{C}}^{n}\right]=1$, we can confirm in Eq. (2.14a) that the notation for $\mathcal{Z}_{1}$ is chosen consistently.

\footnotetext{
${ }^{1}$ or possibly only $n$ replicas, if the cross-link distribution is not the Deam-Edwards distribution
} 


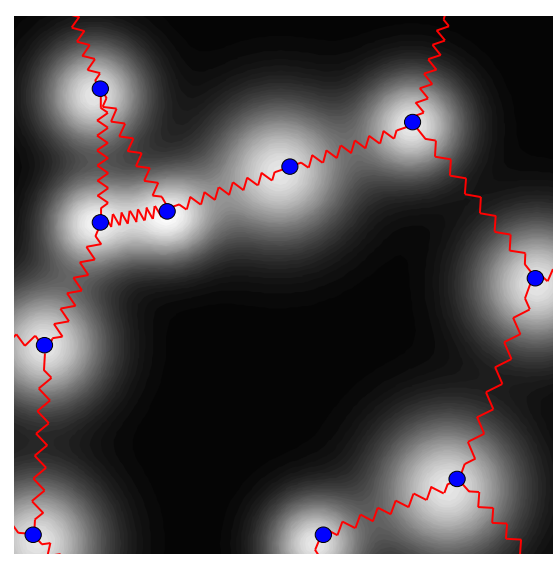

Figure 2.2: Exemplary illustration of a snapshot of the model as in Fig. 2.1, which only contains localized particles. Additionally, the density plot behind the snapshot illustrates, how the thermally averaged density as defined in Eq. (2.15) might look like.

\subsubsection{Introduction of the Replicated Density Fields}

Later we want to introduce a field theory, so sooner or later we have to think about a suitable order parameter. A crucial requirement is that it can distinguish between the liquid and the amorphous solid state. A first idea would be the thermally averaged density

$$
\varrho_{\mathcal{C}}^{(\alpha)}(\mathbf{x}):=\frac{1}{N} \sum_{j=1}^{N}\left\langle\delta\left(\mathbf{x}-\mathbf{r}_{j}^{(\alpha)}\right)\right\rangle_{H_{\mathcal{C}}} .
$$

In the liquid phase, when no macroscopic cluster is present, any particle - even if it is part of a small cluster of connected particles - has equal probability to be found anywhere in the sample; hence this order parameter would simply be a constant. In the gel phase, on the other hand, a macroscopic cluster is present, which is a cluster that contains an infinite number of particles as $N \rightarrow \infty$. A particle of that cluster can not pass through the entire system, because cross-linking to the cluster will restrict its movement to fluctuations around a mean position (as illustrated in Fig. 2.2). Hence we will refer to such a particle as a "localized particle".

The emerging density fluctuations may be utilized to distinguish the gel from the sol phase for a given cross-link configuration $\mathcal{C}$. However, quantities we are interested in, should not be restricted to a certain cross-link configuration, but should be disorder averaged. And when averaging over all cross-link configurations, the macroscopic cluster can be embedded anywhere in the sample and hence the probability to find a particle at any place in the sample should be the same - as in the liquid phase.

To overcome this problem, we take advantage of the fact that the cross-link configuration is the same for all replicas and hence the position of a localized particle should be correlated among the replicas: In the context of gelation, the crucial 
(a)

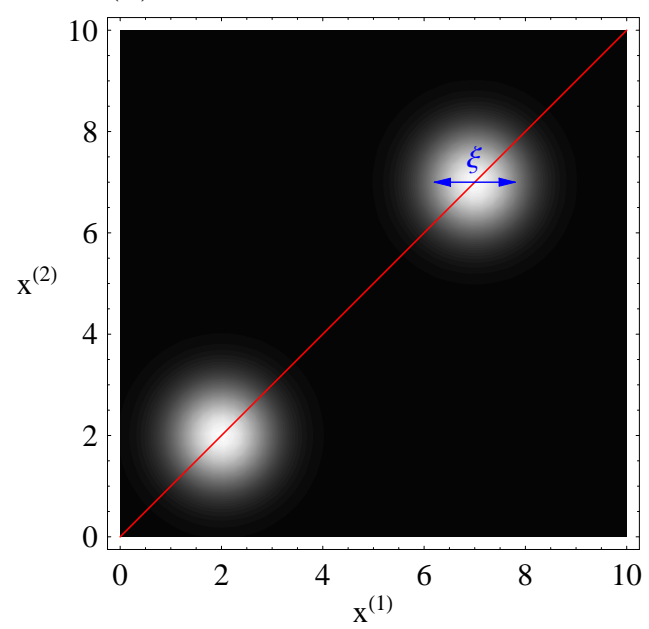

(b)

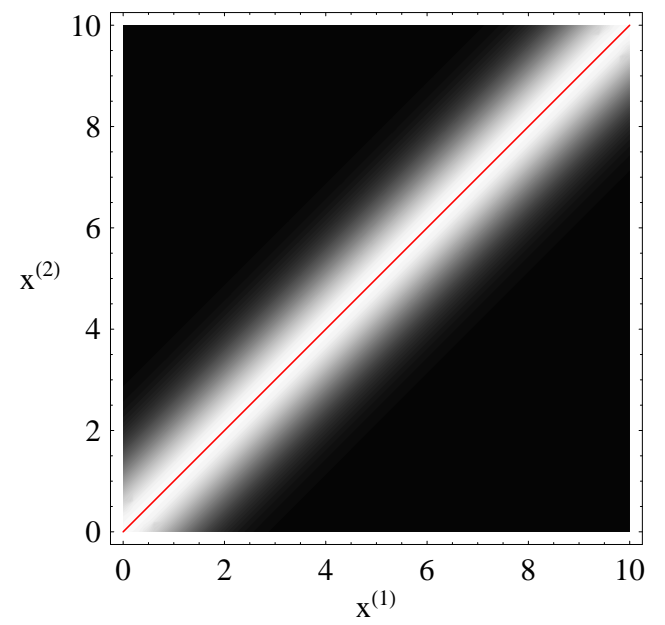

Figure 2.3: For one spatial dimension, the replicated density $\mathrm{O}\left(x^{(1)}, x^{(2)}\right)$, the probability to find a particle at $x^{(1)}$ in replica 1 and at $x^{(2)}$ in replica 2, is illustrated; white means high values. The red line is the angle bisector $x^{(1)}=x^{(2)}$. (a) Example with two localized particles fluctuating around the mean positions $R_{1}=2$ and $R_{2}=7$. The blue arrow $(\leftrightarrow)$ represents the localization length. (b) For the disorder average, we have to integrate the mean positions over the system size. Thus, $\mathrm{O}\left(x^{(1)}, x^{(2)}\right)$ becomes a rectilinear ridge along the axis $x^{(1)}=x^{(2)}$.

question is, whether or not $\left|\mathbf{r}_{j}^{(\alpha)}-\mathbf{r}_{j}^{(\beta)}\right|$, with $\alpha \neq \beta$, stays finite for a certain fraction of particles. With that in mind, we define the replica density:

$$
\mathrm{O}(\hat{x}):=\frac{1}{N} \sum_{j=1}^{N} \delta\left(\hat{x}-\hat{r}_{j}\right)=\frac{1}{N} \sum_{j=1}^{N} \delta\left(\mathbf{x}^{(0)}-\mathbf{r}_{j}^{(0)}\right) \cdots \delta\left(\mathbf{x}^{(n)}-\mathbf{r}_{j}^{(n)}\right)
$$

which is the joint probability to find a particle at $\mathbf{x}^{(0)}$ in replica 0 , and the same particle at $\mathbf{x}^{(1)}$ in replica $1, \ldots$ and at $\mathbf{x}^{(n)}$ in replica $n$. A thermally averaged example of $\mathrm{O}(\hat{x})$, is illustrated in Fig. 2.3a for the case of one spatial dimension and two replicas: A localized particle which is found at position $\mathbf{x}$ in one replica, will have a higher probability to be close to that position also in other replicas. The extent of the particle fluctuations, which we call localization length, is also constituted by the length scale of these correlations.

As before, after performing the disorder average, the mean positions have equal probability to be anywhere in the sample; however, a particle of the macroscopic cluster still has a high probability to be found at close-by positions among the different replicas. This situation is illustrated in Fig. 2.3b, again for one dimension and two replicas: Due to translational invariance, the disorder-averaged replicated density does not depend on the mean position $x_{\mathrm{cm}}=\left(x^{(1)}+x^{(2)}\right) / 2$, but only on the difference $\left(x^{(1)}-x^{(2)}\right) / 2$ between the replicas. That latter dependence still represents the extent of the particle fluctuations $\xi$. In Sec. 2.6 we are going to find 
an Ansatz for the replicated density field and mathematically formulate the aspects introduced here.

For completeness, we also define the replicated density in Fourier space:

$$
\begin{aligned}
\mathrm{O}(\hat{q}) & :=\frac{1}{N} \sum_{j=1}^{N} \exp \left(\mathrm{i} \hat{q} \hat{r}_{j}\right), \\
\mathrm{O}^{(\alpha)}(\mathbf{q}) & :=\frac{1}{N} \sum_{j=1}^{N} \exp \left(\mathbf{i q r}_{j}^{(\alpha)}\right)=\mathrm{O}(\mathbf{0}, \ldots, \underset{\alpha}{\mathbf{q}}, \mathbf{q}, \mathbf{0}, \ldots, \mathbf{0}) .
\end{aligned}
$$

The first definition is the full Fourier transform of Eq. (2.16) and contains correlations between replicas. The latter definition is the ordinary Fourier density of the particle configuration of a given replica $\alpha$.

\subsubsection{Free Energy of the Replicated Density Field}

Now we can go back to Eq. (2.14c) and express the replica free energy in terms of the density fields as introduced in the last section. The calculation is straightforward and done in Appendix B.1.2:

$$
\tilde{f}_{n+1}\left\{\hat{r}_{j}\right\}=f_{0}-\phi^{n} \frac{\mu^{2}}{2} \sum_{\hat{q} \neq \hat{0}}|\mathrm{O}(\hat{q})|^{2} \Delta(\hat{q})+\frac{n_{0} \lambda}{2} \sum_{\alpha=0}^{n} \sum_{\mathbf{q} \neq \mathbf{0}}\left|\mathrm{O}^{(\alpha)}(\mathbf{q})\right|^{2} U(\mathbf{q})
$$

with the mean particle density $n_{0}=N / V$ and the simplifying definitions

$$
\begin{aligned}
f_{0} & :=-\phi^{n} \frac{\mu^{2}}{2}+(n+1) \frac{\lambda n_{0}}{2} \\
\Delta(\hat{q}) & :=\exp \left(-\frac{\hat{q}^{2} a^{2}}{2}\right)
\end{aligned}
$$

The last term of Eq. (2.18) solely comes from the excluded volume interaction. On account of this interaction, density fluctuations cost energy in the same manner in each replica.

In the middle term, the sum also goes over $\hat{q}$-vectors whose components may involve different replicas; hence it comprises the correlations discussed in the previous section. However, at this point it is difficult to see what the immediate effect of this term is. To get a better understanding and to enable further calculations, we introduce different sets of $\hat{q}$-vectors:

We define the one replica sector (1RS) as the set of $\hat{q}$-vectors with exactly one of the $n+1$ components non-zero:

$$
1 \mathrm{RS}=\left\{\hat{q} \neq \hat{0} \mid \hat{q}=\left(\mathbf{0}, \ldots, \mathbf{0}, \mathbf{q}^{(\alpha)}, \mathbf{0}, \ldots, \mathbf{0}\right) \text { with } 0 \leq \alpha \leq n\right\} .
$$

Complementary, the higher replica sector (HRS) is the set of $\hat{q}$-vectors with at least two replica entries non-zero, and therefore all (non-zero) $\hat{q}$-vectors which are not in the 1RS:

$$
\mathrm{HRS}=\{\hat{q} \neq \hat{0} \mid \hat{q} \notin 1 \mathrm{RS}\},
$$


and for the sake of completeness the zero replica sector (0RS) only contains $\hat{q}=\hat{0}$ :

$$
0 \mathrm{RS}=\{\hat{0}\}
$$

With these definitions, Eq. (2.18) becomes:

$$
\begin{aligned}
& \qquad \tilde{f}_{n+1}\left\{\hat{r}_{j}\right\}=f_{0}-\phi^{n} \frac{\mu^{2}}{2} \sum_{\hat{q} \in \mathrm{HRS}}|\mathrm{O}(\hat{q})|^{2} \Delta(\hat{q})+\frac{1}{2} \sum_{\hat{q} \in 1 \mathrm{RS}}|\mathrm{O}(\hat{q})|^{2} \tilde{\lambda}(\hat{q}) \\
& \text { with } \tilde{\lambda}(\hat{q}):=n_{0} \lambda U(\hat{q})-\phi^{n} \mu^{2} \Delta(\hat{q}) .
\end{aligned}
$$

The interpretation of $\tilde{\lambda}(\hat{q})$ is straightforward: In the 1RS (which accounts for density fluctuations) the repulsive excluded volume interaction counteracts the attractive cross-links, see (2.24b). Density fluctuations of wavelength $\hat{q}$ cost the more energy, the higher $\tilde{\lambda}(\hat{q})$ is. As mentioned in the beginning, the strength of excluded volume interaction $\lambda$ has to be large enough to ensure $\tilde{\lambda}(\hat{q})>0$ for all $\hat{q} \in 1$ RS to overcome the attractive cross-link interaction; ${ }^{2}$ otherwise density fluctuations $\mathrm{O}(\hat{q})$ of the corresponding wavelength $\hat{q}$ will be energetically favored and the system will become unstable.

\subsubsection{Introduction of a Field Theory and Decoupling}

It is important to keep in mind that the replicated density $\mathrm{O}(\hat{q})$ is just a simplifying notation. No coarse graining or simplification has been made and in particular, the degrees of freedom of the replica free energy in Eq. (2.24) are still the particle positions $\left\{\hat{r}_{j}\right\}$. Here we will convert the formalism into a field theory, i.e. degrees of freedom shall be constituted by a fluctuating density field, rather than by the particle positions.

In the current state, Eq. (2.24a), the density only appears as a quadratic form. If we somehow could make it linear, i.e. $f\left\{\hat{r}_{j}\right\} \propto \sum_{\hat{q}} a(\hat{q}) \mathrm{O}(\hat{q})$, the partition function would decouple with respect to the particles:

$$
\int\left(\prod_{j=1}^{N} \mathrm{D} \hat{r}_{j}\right) \exp \left(-\sum_{\hat{q}} a(\hat{q}) \sum_{j} \mathrm{e}^{\mathrm{i} \hat{q} \hat{r}_{j}}\right)=\left(\int \mathrm{D} \hat{r} \exp \left(-\sum_{\hat{q}} a(\hat{q}) \mathrm{e}^{\mathrm{i} \hat{q} \hat{r}}\right)\right)^{N}
$$

A way to linearize the quadratic form is the so called Hubbard-Stratonovich transformation. The basic idea is that the linear term of a Gaussian integral becomes quadratic after performing the integral:

$$
\exp \left(k^{2}\right) \propto \int_{-\infty}^{\infty} \mathrm{d} x \exp \left(-x^{2}+k x\right) .
$$

The cost for this linearization is the introduction of the integral over a new variable, in this case $x$. We can make this transformation for each $\hat{q}$-value ${ }^{3}$ and obtain the

\footnotetext{
${ }^{2}$ that requires that $U(\hat{q})$ does not fall off faster than $\Delta(\hat{q})=\exp \left(-a^{2} \hat{q}^{2} / 2\right)$ for $|\hat{q}| \rightarrow \infty$.

${ }^{3}$ and we have to take into account the correct prefactors and that $\mathrm{O}(\hat{q})$ is complex
} 
following result (see Appendix B.2.1 for the calculation):

$$
\mathcal{Z}_{n+1}=\exp \left(-N f_{0}\right) \int \mathcal{D} \Omega \exp \left(-N f_{n+1}\{\Omega\}\right)
$$

with the replica free energy, dependent on the field $\Omega$

$$
f_{n+1}\{\Omega\}=\frac{\phi^{n} \mu^{2}}{2} \sum_{\hat{q} \in \mathrm{HRS}} \Delta(\hat{q})|\Omega(\hat{q})|^{2}+\frac{1}{2} \sum_{\hat{q} \in 1 \mathrm{RS}} \tilde{\lambda}(\hat{q})|\Omega(\hat{q})|^{2}-\ln \mathfrak{z},
$$

with an effective one-particle partition function

$$
\mathfrak{z}=\int \mathrm{D} \hat{r} \exp \left(\phi^{n} \mu^{2} \sum_{\hat{q} \in \mathrm{HRS}} \Delta(\hat{q}) \Omega(\hat{q}) \mathrm{e}^{-\mathrm{i} \hat{q} \hat{r}}+\mathrm{i} \sum_{\hat{q} \in 1 \mathrm{RS}} \tilde{\lambda}(\hat{q}) \Omega(\hat{q}) \mathrm{e}^{-\mathrm{i} \hat{q} \hat{r}}\right),
$$

and a constant contribution $f_{0}$ as in Eq. (2.19). The field $\Omega(\hat{q})$ runs through every possible configuration, via the newly introduced integral in Eq. (2.27a), with the measure $\mathcal{D} \Omega \propto \prod_{\hat{q}} \mathrm{~d} \Omega(\hat{q})$. The interpretation of the field $\Omega(\hat{q})$ is the same as the original density $\mathrm{O}(\hat{q})$, since the difference between them vanishes in the thermodynamic limit (as seen in Appendix B.2.2). Specifically $\Omega(\hat{q})=\mathrm{O}(\hat{q})$ for $\hat{q} \in$ HRS and $\Omega(\hat{q})=\mathrm{iO}(\hat{q})$ for $\hat{q} \in 1 \mathrm{RS}$. Furthermore, as anticipated in Eq. (2.25), the integral over all particle positions is reduced to a single integral over one "effective" particle in Eq. (2.27c).

This is an important stage of the calculation, because so far no approximation has been made. In this work we are not interested in density fluctuations which would be caused by a finite bulk modulus. Therefore, we now take the limit of infinitely strong excluded volume interaction, $\lambda \rightarrow \infty$ and thus $\tilde{\lambda}(\hat{q}) \rightarrow \infty$, to prevent any density fluctuations, i.e. $\Omega(\hat{q} \in 1 \mathrm{RS})=0$. The resulting replica free energy then has a simple form, which only involves the higher replica sector:

$$
\begin{array}{r}
f_{n+1}\{\Omega\}=\frac{\phi^{n} \mu^{2}}{2} \sum_{\hat{q} \in \mathrm{HRS}} \Delta(\hat{q})|\Omega(\hat{q})|^{2}-\ln \mathfrak{z}, \\
\text { with } \mathfrak{z}=\int \mathrm{D} \hat{r} \exp \left(\phi^{n} \mu^{2} \sum_{\hat{q} \in \mathrm{HRS}} \Delta(\hat{q}) \Omega(\hat{q}) \mathrm{e}^{-\mathrm{i} \hat{q} \hat{r}}\right)
\end{array}
$$

\subsubsection{Universality and Connection to Other Models}

For many systems in statistical mechanics it is possible to establish a Landau-Wilson free energy, which depends on a coarse-grained order parameter. A famous example is the Ising model with the order parameter being the coarse-grained magnetization [Goldenfeld, 1992, Chapter 5]. If this order parameter vanishes as a phase transition is approached, one often expands the Landau-Wilson free energy to lowest necessary order. 
As discussed before in Sec. 2.4.2, the replicated density $\Omega(\hat{q})$ is an order parameter that can distinguish between the solid and liquid phase. In the liquid phase $\Omega(\hat{q} \neq \hat{0})=0$, and close to the transition in the gel phase, we may suspect $\Omega(\hat{q} \neq \hat{0})$ to be small, since most of the particles are delocalized and do not give rise to correlations between replicas. Hence we can establish a Ginzburg-Landau like expansion of our model. This expansion of $f_{n+1}\{\Omega\}$, Eq. (2.28), can easily be done (see Appendix B.3). To lowest necessary order in $\Omega$ and $\hat{q}$ ( $\hat{q}$ corresponding to gradients in real-space representation), the replica free energy is:

$$
\begin{aligned}
f_{n+1}\{\Omega\}= & \sum_{\hat{q} \in \mathrm{HRS}}\left(-A \varepsilon+\frac{B}{2} \hat{q}^{2}\right)|\Omega(\hat{q})|^{2} \\
& -C \sum_{\hat{q}_{1}, \hat{q}_{2}, \hat{q}_{3} \in \mathrm{HRS}} \Omega\left(\hat{q}_{1}\right) \Omega\left(\hat{q}_{2}\right) \Omega\left(\hat{q}_{3}\right) \delta_{\hat{q}_{1}+\hat{q}_{2}+\hat{q}_{3}, \hat{0}}+\mathcal{O}\left(\Omega^{4}, \Omega^{3} \hat{q}^{2}, \Omega^{2} \hat{q}^{4}\right)
\end{aligned}
$$

with

$$
\varepsilon=\mu^{2}-1, \quad A=\frac{\mu^{2}}{2}, \quad B=\left(\mu^{4}-\frac{\mu^{2}}{2}\right) a^{2}, \quad C=\frac{\mu^{6}}{6} .
$$

In this simple form (2.29), one can already understand some basic properties of the system: the sol-gel transition happens when the parameter $\varepsilon=\mu^{2}-1$ changes sign: For $\varepsilon<0, \Omega(\hat{q})=0$ is a stable solution, since the prefactor of the quadratic term is positive and any small deviation from this solution costs more energy. For $\varepsilon \gtrsim 0$, this solution becomes unstable and small deviations from $\Omega(\hat{q})=0$ are energetically favorable, whereby the cubic term is necessary to stabilize the new solution. The gradient term $\left(\propto \hat{q}^{2}|\Omega(\hat{q})|^{2}\right)$ is the lowest order that involves spatial dependencies; close to the transition, the relevant length scales of the system (like the above-mentioned localization length) typically diverge. Hence $\Omega(\hat{q})$ should be very small for large $|\hat{q}|$, which justifies neglecting higher orders of $\hat{q}$ close to the transition.

Eq. (2.29) is a universal free energy of an incompressible random system close to the sol-gel transition: This form can also be obtained by pure symmetry considerations and can be used as a phenomenological starting point for the replica free energy [Peng et al., 1998]. With the randomly cross-linked particle model one has a very simple microscopic model that can reproduce this phenomenological free energy and can provide values for the constants $A, B$ and $C$. Other models, like cross-linked semiflexible chains, also reproduce Eq. (2.29) with different values for $A, B$ and $C$ [Goldbart et al., 2004; Mukhopadhyay et al., 2004]. Later we will perform this expansion to infinite order to see how this model behaves far from the gelation transition.

\subsection{Goldstone Fluctuations in General}

In the next section, we will see that the saddle point solution for $\Omega(\hat{q})$ has a continuous symmetry; specifically that means that there is a whole family of saddle-point 
solutions, which are parameterized by homogeneous translations of the entire system between the replicas. We want to establish an Ansatz for the order parameter, which allows small long-wavelength and low-energy fluctuations (so called Goldstone fluctuations) around the saddle point.

Before getting to this Ansatz, it is helpful to have a rough understanding of Goldstone fluctuations in general (see also e.g. [Goldenfeld, 1992, chapter 11] or [Goldstone et al., 1962]). In this regard, let us first consider these Goldstone modes for an easier model, the so called $X Y$-model: In that model, two-dimensional spins $\mathbf{s}_{i}$ of unit length can rotate in a plane and reside on a $D$-dimension lattice (where $D$ can be different from 2); they interact such that neighboring spins (" $<>$ ") preferably align:

$$
H\left(\left\{\mathbf{s}_{i}\right\}\right)=-J \sum_{<i, j>} \mathbf{s}_{i} \cdot \mathbf{s}_{j}=-J \sum_{<i, j>} \cos \left(\theta_{i}-\theta_{j}\right),
$$

where $\theta_{i}$ is the angle of spin $i$ with respect to an arbitrary axis. $J>0$ is the strength of the interaction or can be interpreted as the inverse temperature. One can easily see that in the ground state, all spins point in the same direction.

The important feature for us is that the system has a continuous symmetry, i.e. all spins can continuously be rotated at the same time without energy cost. Or in other words, the transformation $\theta_{i} \rightarrow \theta_{i}+\phi$ for all $i$ and arbitrary $\phi$ leaves the Hamiltonian (2.31) invariant.

For $D>2$, spontaneous symmetry breaking occurs when reducing the temperature below a critical value $T_{\mathrm{c}}$ [Goldenfeld, 1992]. In that case, the system chooses a direction, along which all spins preferably align, yielding a non-zero magnetization $\mathbf{m}=\frac{1}{N} \sum_{i=1}^{N} \mathbf{s}_{i} \neq \mathbf{0}$ (see Fig. 2.4, left). On account of the abovementioned continuous symmetry of the Hamiltonian, this direction can be rotated without energy cost (for example by applying an infinitesimal field).

What about the case that the axis of magnetization is slowly varying in space (Fig. 2.4, right)? Reasoning by continuity, one might expect that the energy associated with these long-wavelength fluctuations of the angle of the spins have low energy cost. Indeed one finds that in the long-wavelength limit, the cost of these fluctuations vanishes with the wave vector $\mathbf{k}$ like $\mathcal{O}\left(\mathbf{k}^{2}\right)$.

These long-wavelength and low-energy modes associated with a continuous symmetry are the Goldstone modes. They are a general phenomenon described by Goldstone's theorem, which states that in the presence of a spontaneously broken continuous symmetry there are long-wavelength excitations, whose energy vanishes continuously in the limit $\mathbf{k} \rightarrow \mathbf{0}$.

\subsection{Ansatz for the Order Parameter}

In Sec. 2.4 we calculated the disorder-averaged free energy $\left[F_{\mathcal{C}}\right]=-\left[\ln Z_{\mathcal{C}}\right]=$ $-\lim _{n \rightarrow 0}\left(\mathcal{Z}_{n+1}-\mathcal{Z}_{1}\right) /\left(n \mathcal{Z}_{1}\right)$ with help of the replica technique. The replica partition function $\mathcal{Z}_{n+1} \propto \int \mathcal{D} \Omega \mathrm{e}^{-N f_{n+1}\{\Omega\}}$ is represented as a functional integral over the order-parameter field $\Omega$. 

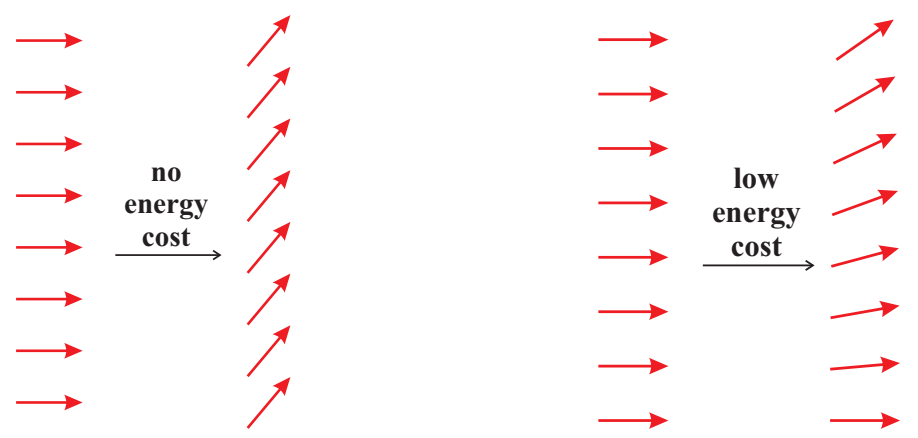

Figure 2.4: Illustration of the $X Y$-model. Only one spatial dimension of the lattice is shown and spins are depicted as red arrows $(\rightarrow)$. Left: A rotation of all spins by a certain angle is an exact symmetry of the Hamiltonian (2.31) and has no energy cost. Right: If the rotation is slowly varying in space, i.e. a long-wavelength fluctuation, the energy is arbitrarily low.

As seen in Appendix B.2.2, in the thermodynamic limit this order parameter equals the replicated density $\mathrm{O}(\hat{q})$ already addressed in Sec. 2.4.2. The real-space representation of the order parameter

$$
\Omega(\hat{x})=\frac{1}{N} \sum_{i=1}^{N} \delta\left(\mathbf{x}^{(0)}-\mathbf{r}_{i}^{(0)}\right) \cdots \delta\left(\mathbf{x}^{(n)}-\mathbf{r}_{i}^{(n)}\right)
$$

quantifies the probability of finding a particle at position $\mathbf{x}^{(0)}$ in replica 0 , at $\mathbf{x}^{(1)}$ in replica 1 , etc.

In previous work, Castillo et al. [1994] have shown that the saddle-point solution for the order parameter, obtained by setting $\delta f_{n+1}\{\Omega\} / \delta \Omega=0$, has the following simple form ${ }^{4}$ :

$$
\bar{\Omega}_{\mathrm{sp}}(\hat{x})=\frac{1-Q}{V^{n+1}}+\frac{Q}{V} \int_{V} \mathrm{~d} \mathbf{y} \int_{0}^{\infty} \frac{\mathrm{d} \xi^{2} \mathcal{P}\left(\xi^{2}\right)}{\left(2 \pi \xi^{2}\right)^{D(n+1) / 2}} \exp \left(-\sum_{\alpha=0}^{n} \frac{\left(\mathbf{x}^{(\alpha)}-\mathbf{y}\right)^{2}}{2 \xi^{2}}\right),
$$

with self-consistent equations for $Q$ and $\mathcal{P}\left(\xi^{2}\right)$ given in [Castillo et al., 1994; Broderix et al., 2002]. This form has a very intuitive interpretation, since the following premises, applied to Eq. (2.32), also reproduce (2.33): A fraction of monomers $(1-Q)$ is delocalized and can be found anywhere in the sample with equal probability. The remaining fraction $Q$ is the "infinite" cluster, in which each particle $i$ performs Gaussian fluctuations about a replica-independent mean position $\mathbf{y}_{i}$ with localization length $\xi_{i}$. (The situation is qualitatively depicted in Fig. 2.3a in Sec. 2.4.2.) The mean positions $\mathbf{y}_{i}$ are randomly distributed (Fig. 2.3b), and the localization lengths $\xi$ follow the distribution $\mathcal{P}\left(\xi^{2}\right)$.

\footnotetext{
${ }^{4}$ Castillo et al. [1994] use a slightly different model of cross-linked chains, which belongs to the same universality class as the presented model. The saddle-point value obtained for our model is the same.
} 


\subsubsection{Spontaneously Broken Translational Invariance}

The effective replicated Hamiltonian is invariant under uniform translations of all particles, separately in each replica, see e.g. Eq. (2.14c), since the particle positions only appear in terms of the form $\left(\mathbf{r}_{i}^{(\alpha)}-\mathbf{r}_{j}^{(\alpha)}\right)$; i.e., the transformation

$$
\mathbf{r}_{i}^{(\alpha)} \rightarrow \mathbf{r}_{i}^{(\alpha)}+\mathbf{u}^{(\alpha)}
$$

is an exact symmetry of the Hamiltonian. ${ }^{5}$

Now, let us have a look at the saddle point solution (2.33): In the amorphous solid phase, $Q>0$, this symmetry is spontaneously broken, and the transformation (2.34), in general, yields a different order parameter; the only symmetries remaining are common displacements of all replicas, i.e., $\mathbf{r}_{i}^{(\alpha)} \rightarrow \mathbf{r}_{i}^{(\alpha)}+\mathbf{u}$, reflecting the macroscopic translational invariance of the amorphous solid phase. (For a detailed discussion see [Goldbart et al., 2004; Mukhopadhyay et al., 2004].) Consequently, there is a whole family of order parameters which are all related by replicadependent, spatially uniform translations. All of them give rise to the same free energy and the system will "spontaneously" choose one of them. To distinguish common and relative displacements of the replicas, it is helpful to split up the $D(n+1)$-dimensional hatted vectors $\hat{x}$ into $D$-dimensional longitudinal ones

$$
\mathbf{x}_{\|}=\frac{1}{\sqrt{n+1}} \sum_{\alpha=0}^{n} \mathbf{x}^{(\alpha)}
$$

and $D n$-dimensional transverse ones

$$
x_{\perp}=\hat{x}-\frac{1}{\sqrt{n+1}}\left(\mathbf{x}_{\|}, \ldots, \mathbf{x}_{\|}\right) .
$$

Properties of this division into $\|$ - and $\perp$-vectors can be found in Appendix A. A very useful relation to keep in mind is

$$
\hat{x} \cdot \hat{y}=\mathbf{x}_{\|} \cdot \mathbf{y}_{\|}+x_{\perp} \cdot y_{\perp} .
$$

When considering a parallel-vector like $\mathbf{x}_{\|}$, one should keep in mind that it is not exactly the center of mass $\mathbf{x}_{\mathrm{cm}}=\frac{1}{n+1} \sum_{\alpha} \mathbf{x}^{(\alpha)}$ of its replica components, but $\mathbf{x}_{\|}=\sqrt{n+1} \mathbf{x}_{\mathrm{cm}}$. Only this definition enables the simple relation (2.37). As a consequence, $\|$-vectors reside in a different volume $V_{\|}:=V(n+1)^{D / 2}$, rather than $V$. For consistency, we also define a parallel-vector $\mathbf{R}_{\|}:=\sqrt{n+1} \mathbf{R}$ for any non-replicated vector $\mathbf{R}$.

With the help of these definitions we can parameterize the manifold of symmetryrelated saddle points by $u_{\perp}$ :

$$
\bar{\Omega}_{u_{\perp}}(\hat{x})=\frac{1-Q}{V^{n+1}}+\frac{Q}{V_{\|}} \int_{0}^{\infty} \frac{\mathrm{d} \xi^{2} \mathcal{P}\left(\xi^{2}\right)}{\left(2 \pi \xi^{2}\right)^{D n / 2}} \exp \left(-\frac{\left(x_{\perp}-u_{\perp}\right)^{2}}{2 \xi^{2}}\right),
$$

\footnotetext{
${ }^{5}$ The effective replicated Hamiltonian is also invariant under uniform rotations, separately in each replica. However the spontaneous breaking of this symmetry does not give rise to Goldstone modes and is not discussed further here.
} 
(a)

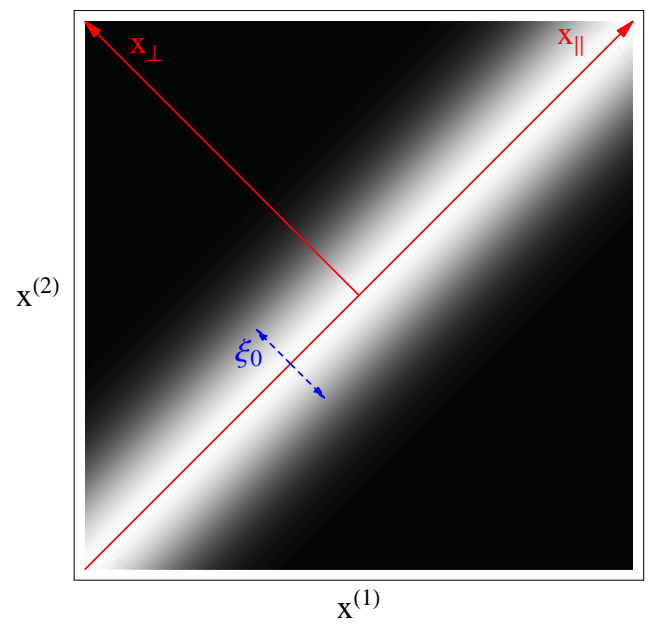

(b)

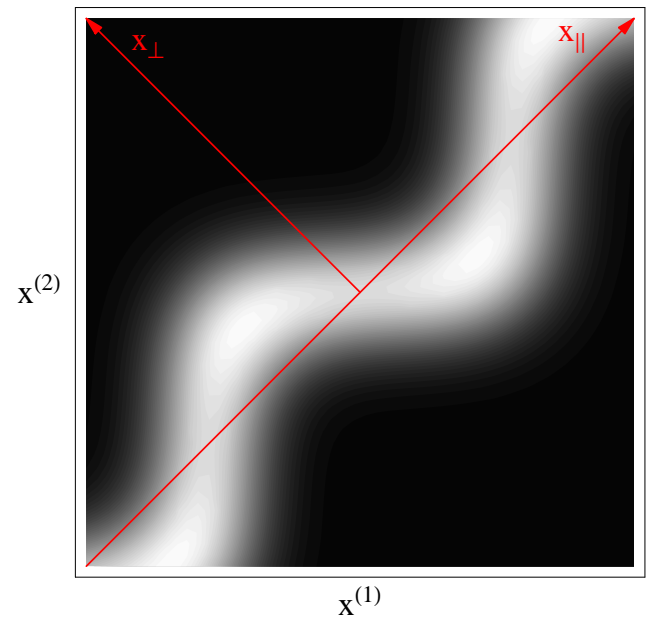

Figure 2.5: Ansatz for the order parameter $\Omega\left(x^{(1)}, x^{(2)}\right)$ in real space for two replicas in one dimension, as Fig. 2.3. To simplify, we have assumed a fixed localization length $\xi_{0}$ in this figure. $\Omega\left(x^{(1)}, x^{(2)}\right)$ is the probability of finding a particle at position $x^{(1)}$ in replica 1 and at $x^{(2)}$ in replica 2 . For the rotated coordinate system $x_{\|}=$ $\left(x^{(2)}+x^{(1)}\right) / \sqrt{2}$ and $x_{\perp}=\left(x^{(2)}-x^{(1)}\right) / 2$. In (a) the displacement vector $u_{\perp} \equiv 0$, and in (b) $u_{\perp}=\sin x_{\|}$. (Note, however, that for physical systems typically $\xi \ll$ wavelength of $u_{\perp}$.)

where $u_{\perp}=\hat{0}$ yields Eq. (2.33). The situation is illustrated in Fig. 2.5a, where the order parameter is shown for two replicas in one space-dimension. The order parameter does not depend on $\mathbf{x}_{\|}$, so that it is represented by a rectilinear ridge having its maximum height along the axis $x_{\perp}=\hat{0}$ and having width $\xi$. The family of symmetry-related solutions is generated by rigidly translating the ridge to a new position: $x_{\perp}=u_{\perp}$. (The delocalized particles contribute only an unimportant constant background $(1-Q) / V^{n+1}$, which has been ignored in the figure.)

\subsubsection{Goldstone Fluctuations and Ansatz for $\Omega$}

Now, we want to establish an Ansatz for the order parameter that allows for special fluctuations around the saddle point. These fluctuations are low-energy and long-wavelength excitations (i.e. Goldstone modes) and are constructed by particle displacements $u_{\perp}\left(\mathbf{x}_{\|}\right)$that depend on the position in the sample [Mukhopadhyay et al., 2004; Goldbart et al., 2004]. These local deformations may be generated by thermal fluctuations in the system. The Ansatz developed in this section was already briefly presented in [Ulrich et al., 2006].

For the more general Ansatz, we make the same basic premises that reproduced the saddle point solution (2.33): The fraction $(1-Q)$ of particles is delocalized, and a particle $j$ of the remaining fraction $Q$ (the gel fraction) performs Gaussian fluctuations around a mean position $\mathbf{R}_{j}^{(\alpha)}$. Now, however, these mean positions 
depend on the replica and may be shifted by a spatially dependent value $\mathbf{u}_{\perp}^{(\alpha)}\left(\mathbf{x}_{\|}\right)$ from one another between the replicas, such that $\mathbf{R}_{j}^{(\alpha)}=\mathbf{R}_{j}+\mathbf{u}_{\perp}^{(\alpha)}\left(\mathbf{R}_{j \|}\right)$. Hence the probability to find particle $j$ at position $\mathbf{x}$ in replica $\alpha$ is

$$
\begin{aligned}
& \left\langle\delta\left(\mathbf{x}-\mathbf{r}_{j}^{(\alpha)}\right)\right\rangle= \\
& = \begin{cases}\frac{1}{\left(2 \pi \xi_{j}^{2}\right)^{D / 2}} \exp \left(-\frac{\left(\mathbf{x}-\left(\mathbf{R}_{j}+\mathbf{u}_{\perp}^{(\alpha)}\left(\mathbf{R}_{j \|}\right)\right)^{2}\right.}{2 \xi_{j}^{2}}\right) & \text { if particle } j \text { localized (loc.) } \\
1 / V & \text { if particle } j \text { delocalized (deloc.) }\end{cases}
\end{aligned}
$$

and thus, for all replicas, the joint probability density to find a particle at $\mathbf{x}^{(0)}$ in replica (0), at $\mathbf{x}^{(1)}$ in replica (1), etc. is

$$
\left\langle\delta\left(\hat{x}-\hat{r}_{j}\right)\right\rangle= \begin{cases}\frac{1}{\left(2 \pi \xi_{j}^{2}\right)^{D(n+1) / 2}} \exp \left(-\frac{\left(\hat{x}-\hat{R}_{j}\right)^{2}}{2 \xi_{j}^{2}}\right) & \text { if } j \text { loc. } \\ V^{-(n+1)} & \text { if } j \text { deloc. }\end{cases}
$$

with $\hat{R}_{j}=\left(\mathbf{R}_{j}^{(0)}, \ldots, \mathbf{R}_{j}^{(n)}\right)=\left(\mathbf{R}_{j}+\mathbf{u}_{\perp}^{(0)}\left(\mathbf{R}_{j \|}\right), \ldots, \mathbf{R}_{j}+\mathbf{u}_{\perp}^{(n)}\left(\mathbf{R}_{j \|}\right)\right)$. The Fourier transform of this distribution is given by:

$$
\begin{aligned}
& \left\langle\exp \left(\mathrm{i} \hat{q} \hat{r}_{j}\right)\right\rangle= \\
& \quad=\int \mathrm{d} \hat{x} \exp (\mathrm{i} \hat{q} \hat{x})\left\langle\delta\left(\hat{x}-\hat{r}_{j}\right)\right\rangle= \begin{cases}\exp \left(-\frac{\hat{q}^{2} \xi_{j}^{2}}{2}+\mathrm{i} \hat{q} \hat{R}_{j}\right) & \text { if } j \text { loc. } \\
\delta_{\hat{q}, \hat{0}} & \text { if } j \text { deloc. }\end{cases}
\end{aligned}
$$

Hence, we get for the averaged order parameter:

$$
\begin{aligned}
\langle\Omega(\hat{q})\rangle & =\frac{1}{N} \sum_{j=1}^{N}\left\langle\exp \left(\mathrm{i} \hat{q} \hat{r}_{j}\right)\right\rangle \\
& =\frac{1}{N} \sum_{j \text { loc. }} \exp \left(-\frac{\hat{q}^{2} \xi_{j}^{2}}{2}+\mathrm{i} \sum_{\alpha=0}^{n} \mathbf{q}^{(\alpha)}\left(\mathbf{R}_{j}+\mathbf{u}_{\perp}^{(\alpha)}\left(\mathbf{R}_{j \|}\right)\right)\right)+\frac{1}{\not} \cdot \not X(1-Q) \delta_{\hat{q}, \hat{0}}
\end{aligned}
$$

Now, to incorporate the disorder average, we assume a uniform distribution of the undeformed mean positions $\mathbf{R}_{j}$. We also define the distribution for the localization lengths

$$
\mathcal{P}\left(\xi^{2}\right)=\frac{1}{Q N} \sum_{j \text { loc. }} \delta\left(\xi^{2}-\xi_{j}^{2}\right)
$$

and get:

$$
\begin{aligned}
\bar{\Omega}_{u}(\hat{q}) & -(1-Q) \delta_{\hat{q}, \hat{0}} \\
= & \frac{1}{N} \sum_{j \text { loc. }} \exp \left(-\frac{\hat{q}^{2} \xi_{j}^{2}}{2}\right) \int \frac{\mathrm{d} \mathbf{R}_{j}}{V} \exp \left(\mathrm{i} \sum_{\alpha=0}^{n} \mathbf{q}^{(\alpha)}\left(\mathbf{R}_{j}+\mathbf{u}_{\perp}^{(\alpha)}\left(\mathbf{R}_{j \|}\right)\right)\right) \\
= & \frac{Q}{V_{\|}} \int_{0}^{\infty} \mathrm{d} \xi^{2} \mathcal{P}\left(\xi^{2}\right) \exp \left(-\frac{\hat{q}^{2} \xi^{2}}{2}\right) \int \mathrm{d} \mathbf{x}_{\|} \exp \left(\mathrm{i} \mathbf{q}_{\|} \mathbf{x}_{\|}+\mathrm{i} q_{\perp} u_{\perp}\left(\mathbf{x}_{\|}\right)\right)
\end{aligned}
$$


which can be written in a simple way:

$$
\begin{gathered}
\bar{\Omega}_{u}(\hat{q})=(1-Q) \delta_{\hat{q}, \hat{0}}+Q \omega(\hat{q}) \int \operatorname{Dx} \mathbf{x}_{\|} \exp \left(\mathbf{i} \mathbf{q}_{\|} \mathbf{x}_{\|}+\mathrm{i} q_{\perp} u_{\perp}\left(\mathbf{x}_{\|}\right)\right) \\
\text {with } \quad \omega(\hat{q})=\int_{0}^{\infty} \mathrm{d} \xi^{2} \mathcal{P}\left(\xi^{2}\right) \exp \left(-\frac{\hat{q}^{2} \xi^{2}}{2}\right)
\end{gathered}
$$

and with the dimensionless measure $\mathrm{D} \mathbf{x}_{\|}=\mathrm{d} \mathbf{x}_{\|} / V_{\|}$. In real space this result transforms into

$$
\begin{aligned}
\bar{\Omega}_{u}(\hat{x})=\frac{1-Q}{V^{n+1}}+Q & \int_{0}^{\infty} \frac{\mathrm{d} \xi^{2} \mathcal{P}\left(\xi^{2}\right)}{\left(2 \pi \xi^{2}\right)^{D(n+1) / 2}} \\
& \times \int \operatorname{Dy}_{\|} \exp \left(-\frac{\left(\mathbf{y}_{\|}-\mathbf{x}_{\|}\right)^{2}+\left(x_{\perp}-u_{\perp}\left(\mathbf{y}_{\|}\right)\right)^{2}}{2 \xi^{2}}\right)
\end{aligned}
$$

This form is illustrated in Fig. 2.5b: Unlike the homogeneous translations in Eq. (2.38) and Fig. 2.5a, the ridge is not translated rigidly, but deformed by the position-dependent vector $u_{\perp}\left(\mathbf{x}_{\|}\right)$. These excitations $d o$ cost energy; only in the long-wavelength limit do we expect the energy of the excitation to vanish with an inverse power of the wavelength.

We only consider pure shear deformations, and hence require that $\left|\operatorname{det}\left(\delta_{\tau \nu}+\partial_{\tau} u_{\perp}^{(\alpha, \nu)}\right)\right|=1$. This constraint is consistent with taking the excluded volume interaction to be large, which makes the system incompressible. For small deformations, the constraint simplifies to $\partial_{\nu} u_{\perp}^{(\alpha, \nu)}=0$.

It is important to note that the deformations in the Ansatz, Eq. (2.45), represent small fluctuations around the saddle-point solution (2.33). As we see in Appendix B.4, in the limit of small deformations $u_{\perp}$ and long wavelengths (i.e. small $\hat{q}$ ), the Ansatz can be expressed as

$$
\bar{\Omega}_{u}(\hat{q})=\bar{\Omega}_{\mathrm{sp}}(\hat{q})+\delta \Omega_{u}(\hat{q})
$$

with a small $\delta \Omega_{u}(\hat{q})=\mathcal{O}\left(q_{\perp} u_{\perp}\right)$. Consequently, for $u_{\perp}\left(\mathbf{x}_{\|}\right) \equiv \hat{0}$, the Ansatz reproduces the saddle-point solution (2.33) (here in Fourier representation):

$$
\bar{\Omega}_{u \equiv 0}(\hat{q}) \equiv \bar{\Omega}_{\mathrm{sp}}(\hat{q})=(1-Q) \delta_{\hat{q}, \hat{0}}+Q \omega(\hat{q}) \delta_{\mathbf{q}_{\|}, \mathbf{0}} .
$$

\subsubsection{Physical Shear Deformations}

Here we examine what the physical meaning of the shear deformation field $u_{\perp}\left(\mathbf{x}_{\|}\right)$is. Let us first assume, there is a particle at position $\mathbf{x}$ in the unsheared state $\left(u_{\perp}=\hat{0}\right.$, see Fig. 2.6, left). If there is a non-zero $u_{\perp}\left(\mathbf{x}_{\|}\right)$, this particle will be moved to the position $\mathbf{x}+\mathbf{u}_{\perp}^{(0)}\left(\mathbf{x}_{\|}\right)$in the zeroth replica. In this replica cross-linking takes place, 


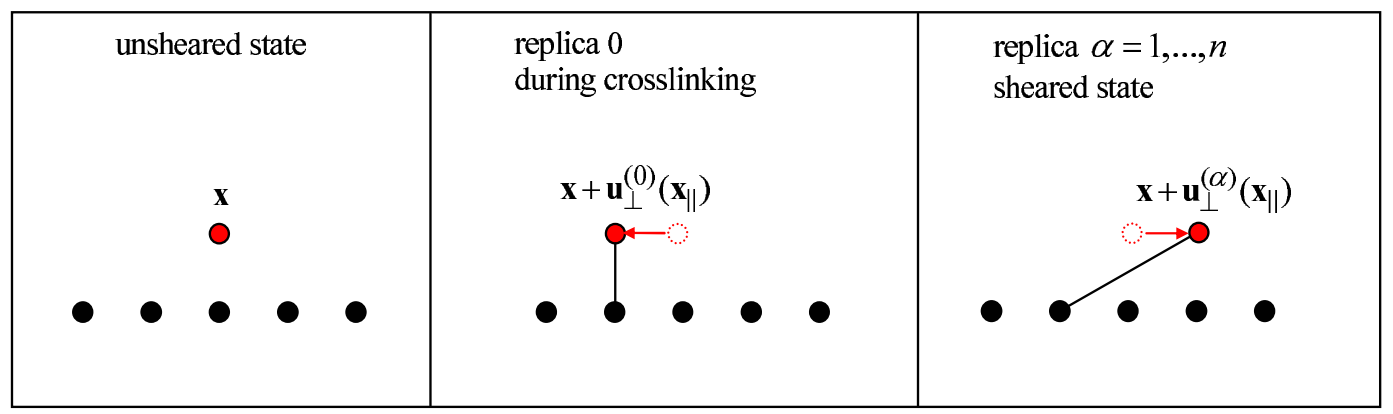

Figure 2.6: Descriptive illustration of the deformation vector $u_{\perp}\left(\mathbf{x}_{\|}\right)$. Left: In the unsheared state, there is a particle at position $\mathbf{x}$. Center: In the zeroth replica the deformation causes the particle to be at the position $\mathbf{x}+\mathbf{u}_{\perp}^{(0)}\left(\mathbf{x}_{\|}\right)$. It will preferably be cross-linked to particles close to that position. Right: In replicas $\alpha=1, \ldots, n$, that particle is moved to $\mathbf{x}+\mathbf{u}_{\perp}^{(\alpha)}\left(\mathbf{x}_{\|}\right)$, but still cross-linked to particles that were close in replica (0).

hence this particle will preferably be cross-linked to particles close to that position (Fig. 2.6, center). The measurement is performed in replicas $\alpha=1, \ldots, n$, in which the particle is moved to the position $\mathbf{x}+\mathbf{u}_{\perp}^{(\alpha)}\left(\mathbf{x}_{\|}\right)$(Fig. 2.6, right). However, the physically relevant deformation is the displacement of this particle relative to the state during cross-linking, $\mathbf{u}_{\text {phys }}^{(\alpha)}\left(\mathbf{x}_{\|}\right)=\mathbf{u}_{\perp}^{(\alpha)}\left(\mathbf{x}_{\|}\right)-\mathbf{u}_{\perp}^{(0)}\left(\mathbf{x}_{\|}\right)$. Hence it is useful to introduce the physical deformation field

$$
\mathbf{u}_{\text {phys }}^{(\alpha)}\left(\mathbf{x}_{\|}\right):=\mathbf{u}_{\perp}^{(\alpha)}\left(\mathbf{x}_{\|}\right)-\mathbf{u}_{\perp}^{(0)}\left(\mathbf{x}_{\|}\right), \quad \alpha=0, \ldots, n
$$

which has $n$ non-zero components and contains the same information as $u_{\perp}\left(\mathbf{x}_{\|}\right)$.

One can obtain a useful relation between the two fields:

$$
\sum_{\alpha=0}^{n}\left(\frac{\partial u_{\perp}^{(\alpha, \nu)}}{\partial x_{\|}^{(\tau)}}\right)^{2}=\sum_{\alpha=1}^{n}\left(\frac{\partial u_{\mathrm{phys}}^{(\alpha, \nu)}}{\partial x_{\|}^{(\tau)}}\right)^{2}-\frac{1}{n+1}\left(\sum_{\alpha=1}^{n} \frac{\partial u_{\mathrm{phys}}^{(\alpha, \nu)}}{\partial x_{\|}^{(\tau)}}\right)^{2},
$$

which can easily be proved by plugging in the definition of $\mathbf{u}_{\text {phys }}^{(\alpha)}\left(\mathbf{x}_{\|}\right)$, Eq. (2.49). In the above equation $\nu, \tau=1, \ldots, D$ are spatial indices. We note that the sum of the last term is of the form $\left(\sum_{\alpha=1}^{n} \ldots\right)^{2}=\mathcal{O}\left(n^{2}\right)$ and therefore we can write in the limit $n \rightarrow 0$ :

$$
\sum_{\alpha=0}^{n}\left(\frac{\partial u_{\perp}^{(\alpha, \nu)}}{\partial x_{\|}^{(\tau)}}\right)^{2}=\sum_{\alpha=1}^{n}\left(\frac{\partial u_{\mathrm{phys}}^{(\alpha, \nu)}}{\partial x_{\|}^{(\tau)}}\right)^{2}+\mathcal{O}\left(n^{2}\right)
$$

\subsection{Replica Free Energy with Shear Deformations}

Now we will insert the Ansatz (2.45) into the general expression (2.28) for the free energy and thereby allow for physically motivated fluctuations (namely the local 
shear displacements $\left.u_{\perp}\left(\mathbf{x}_{\|}\right)\right)$around the saddle point:

$$
\frac{\mathcal{Z}_{n+1}}{\exp \left(-N f_{0}\right)}=\int \mathcal{D} \Omega \exp \left(-N f_{n+1}\{\Omega\}\right) \approx \int \mathcal{D} u \exp \left(-N f_{n+1}\left\{\bar{\Omega}_{u}\right\}\right)
$$

The integral $\int \mathcal{D} u$ goes over all possibilities of the local shear field, separately in all replicas.

In this approach, we will only consider small, long-wavelength distortions, so that it is sufficient to keep $u_{\perp}$ only to the lowest order, $\left(\partial u_{\perp} / \partial \mathbf{x}_{\|}\right)^{2}$. Higher orders in $u_{\perp}$ and higher-order derivatives will be neglected. The calculation is somewhat intricate and can be found in Appendix B.5. The result for the replica free energy can be written in a very concise way as:

$$
N f_{n+1}\left\{\bar{\Omega}_{u}\right\}=N f_{n+1}^{\mathrm{sp}}+\frac{G}{2} \int \mathrm{d} \mathbf{x}_{\|} \sum_{\alpha=0}^{n} \sum_{\tau, \nu=1}^{D}\left(\frac{\partial u_{\perp}^{(\alpha, \nu)}\left(\mathbf{x}_{\|}\right)}{\partial x_{\|}^{(\tau)}}\right)^{2}+\mathcal{O}\left(n^{2}\right)
$$

where the first term, $f_{n+1}^{\mathrm{sp}}$, is the saddle-point value of $f_{n+1}\{\Omega\}$ :

$$
\begin{aligned}
f_{n+1}^{\mathrm{sp}}=n\left\{\frac{D}{2}\left(\exp \left(-\mu^{2} Q\right)+\mu^{2} Q-1-\frac{\mu^{2} Q^{2}}{2}\right)\right. & \\
+ & \left.\frac{\mu^{2} Q^{2}}{2} \Xi_{2, a^{2} / 2}-\exp \left(-\mu^{2} Q\right) \sum_{r=2}^{\infty} \frac{\left(\mu^{2} Q\right)^{r}}{r !} \Xi_{r, a^{2}}\right\}
\end{aligned}
$$

and the second term describes the cost of fluctuations (i.e. shear deformations) around the saddle point, with the shear modulus

$$
G=\left(\exp \left(-\mu^{2} Q\right)+\mu^{2} Q-1-\frac{\mu^{2} Q^{2}}{2}\right) n_{0}
$$

For the saddle-point value Eq. (2.53b), $\Xi_{r, a^{2}}$ is a simplifying definition that depends on the microscopic parameters of the system:

$$
\Xi_{r, a^{2}}:=\int \mathrm{d} \xi_{1}^{2} \mathcal{P}\left(\xi_{1}^{2}\right) \cdots \mathrm{d} \xi_{r}^{2} \mathcal{P}\left(\xi_{r}^{2}\right) \ln \left(\left(\frac{V}{(2 \pi)^{D / 2}}\right)^{r-1}\left(\frac{\prod_{\gamma=1}^{r}\left(a^{2}+\xi_{\gamma}^{2}\right)^{-1}}{\sum_{\gamma=1}^{r}\left(a^{2}+\xi_{\gamma}^{2}\right)^{-1}}\right)^{D / 2}\right) .
$$

In the thermodynamic limit, when $V^{1 / D} \gg \xi, a$, the dependence on $\xi$ and $a$ vanishes, and $\Xi_{r, a^{2}}$ reduces to (see Eq. (B.101) in Appendix B.5.6):

$$
\Xi_{r, a^{2}}=(r-1) \ln N+\mathcal{O}\left(N^{0}\right) .
$$

One has to keep in mind, however, that for some purposes the full form (2.54a) is needed, for example, for determining the distribution of localization lengths $\mathcal{P}\left(\xi^{2}\right)$ 
as e.g. in [Goldbart et al., 1996, Sec. VI C]. For all our purposes, however, the simple Eq. (2.54b) is sufficient. We plug it into Eq. (2.53b) and use (B.93) for the sum over $r$. With that, $f_{n+1}^{\mathrm{sp}}$ becomes very simple:

$$
f_{n+1}^{\mathrm{sp}}=n\left(\exp \left(-\mu^{2} Q\right)+\mu^{2} Q-1-\frac{\mu^{2} Q^{2}}{2}\right)\left(\frac{D^{\prime}}{2}-\ln N+\mathcal{O}\left(N^{0}\right)\right)
$$

It is noteworthy that this saddle point solution of the free energy per particle $f_{n+1}^{\mathrm{sp}}$ is not intensive, but proportional to $\ln N$. This point has already been discussed in [Goldbart et al., 1996]: it is most probably coming from the fact that for a given cross-link configuration $\mathcal{C}$, certain particles are indistinguishable (e.g. if particles 1 , 2 and 3 are connected as a triangle, all three of those particles can be interchanged yielding the same configuration). Therefore, if we insist on an intensive free energy, we have to normalize the partition function by a prefactor taking into account this indistinguishability. However, until now, this could not be done for this or similar models and does not matter if one considers $\partial f / \partial Q=0$.

\subsection{Returning to the Disorder-Averaged Free Energy}

In the last section, we found the replica free energy $f_{n+1}\left\{\bar{\Omega}_{u}\right\}$ and its dependence on the shear deformations $u_{\perp}\left(\mathbf{x}_{\|}\right)$, see Eqs. (2.53). Here we want to establish the connection to the "real" disorder-averaged free energy $\left[F_{\mathcal{C}}\right]$. With Eqs. $(2.14 \mathrm{a})$ and (2.10), we can relate the replicated partition function $\mathcal{Z}_{n+1}$ to the disorder-averaged free energy:

$$
\begin{aligned}
-\left[F_{\mathcal{C}}\right]=\lim _{n \rightarrow 0} \frac{\left[Z_{\mathcal{C}}^{n}\right]-1}{n} & =\lim _{n \rightarrow 0} \frac{\mathcal{Z}_{n+1} / \mathcal{Z}_{1}-1}{n}=\frac{1}{\mathcal{Z}_{1}} \lim _{n \rightarrow 0} \frac{\mathcal{Z}_{n+1}-\mathcal{Z}_{1}}{n} \\
& =\frac{1}{\mathcal{Z}_{1}} \lim _{n \rightarrow 0} \frac{\partial \mathcal{Z}_{n+1}}{\partial n}=\lim _{n \rightarrow 0} \frac{\partial \ln \mathcal{Z}_{n+1}}{\partial n}
\end{aligned}
$$

and hence:

$$
\left[F_{\mathcal{C}}\right]=-\lim _{n \rightarrow 0} \frac{\partial}{\partial n} \ln \mathcal{Z}_{n+1}
$$

Applying this relation to $\mathcal{Z}_{n+1}$ from Eqs. (2.52) and (2.53a), we find:

$$
\begin{aligned}
{\left[F_{\mathcal{C}}\right]=} & N \lim _{n \rightarrow 0} \frac{\partial}{\partial n}\left(f_{0}+f_{n+1}^{\mathrm{sp}}\right) \\
& +\lim _{n \rightarrow 0} \frac{\partial}{\partial n} \ln \int \mathcal{D} u \exp \left(-\frac{G}{2} \sum_{\alpha} \sum_{\nu, \tau} \int \mathrm{d} \mathbf{x}_{\|}\left(\frac{\partial u_{\perp}^{(\alpha, \nu)}\left(\mathbf{x}_{\|}\right)}{\partial x_{\|}^{(\tau)}}\right)^{2}\right) .
\end{aligned}
$$

The only degrees of freedom left are constituted by the displacement field $u_{\perp}\left(\mathbf{x}_{\|}\right)$. However, $u_{\perp}$ contains the mathematically unpleasant constraint that the sum over all replica components has to be zero. With Eq. (2.51), we can relate $u_{\perp}$ to the 
physical shear deformation $\hat{u}_{\text {phys }}$ and write the integral $\int \mathcal{D} u$ as an integral over the $n$ components of $\hat{u}_{\text {phys }}$ :

$$
\begin{aligned}
& {\left[F_{\mathcal{C}}\right]-N \lim _{n \rightarrow 0} \frac{\partial\left(f_{0}+f_{n+1}^{\mathrm{sp}}\right)}{\partial n}} \\
& =-\lim _{n \rightarrow 0} \frac{\partial}{\partial n} \ln \left\{\int\left(\prod_{\alpha=1}^{n} \mathcal{D} \mathbf{u}_{\mathrm{phys}}^{(\alpha)}\right) \exp \left(-\frac{G}{2} \sum_{\alpha=1}^{n} \sum_{\nu, \tau} \int \mathrm{d} \mathbf{x}_{\|}\left(\frac{\partial u_{\mathrm{phys}}^{(\alpha, \nu)}\left(\mathbf{x}_{\|}\right)}{\partial x_{\|}^{(\tau)}}\right)^{2}\right)\right\} \\
& =-\lim _{n \rightarrow 0} \frac{\partial}{\partial n} \ln \left(\left\{\int \mathcal{D} \mathbf{u}_{\mathrm{phys}} \exp \left(-\frac{G}{2} \sum_{\nu, \tau} \int \mathrm{d} \mathbf{x}_{\|}\left(\frac{\partial u_{\mathrm{phys}}^{(\nu)}\left(\mathbf{x}_{\|}\right)}{\partial x_{\|}^{(\tau)}}\right)^{2}\right)\right\}^{n}\right) .
\end{aligned}
$$

We can get $n$-th power of the curly bracket out of the logarithm, and obtain:

$$
\begin{aligned}
{\left[F_{\mathcal{C}}\right] } & =N \lim _{n \rightarrow 0} \frac{\partial\left(f_{0}+f_{n+1}^{\mathrm{sp}}\right)}{\partial n}-\ln Z^{\mathrm{el}} \\
\text { with } \quad Z^{\mathrm{el}} & =\int \mathcal{D} \mathbf{u}_{\text {phys }} \exp \left(-\frac{G}{2} \sum_{\nu, \tau} \int \mathrm{d} \mathbf{x}\left(\frac{\partial u_{\mathrm{phys}}^{(\nu)}(\mathbf{x})}{\partial x^{(\tau)}}\right)^{2}\right)
\end{aligned}
$$

Hereby, $Z^{\mathrm{el}}$ is the partition function of the energy contribution of an incompressible medium [Landau \& Lifshitz, 1965]. It does not involve replicas anymore, and thus we can confirm that $G$ indeed plays the role of the shear modulus. It is analyzed in more detail in Sec. 2.9.3.

We can see in Eq. (2.60a) that, in addition to the elastic contribution, the linear order in $n$ of the saddle point solution $f_{n+1}^{\mathrm{sp}}$ adds to the disorder-averaged free energy per particle.

\subsection{Results}

\subsubsection{Average Cross-Link Density and Higher Moments}

Given the Deam-Edwards distribution Eq. (2.8), it is possible to calculate expectation values of the form $[M(M-1) \cdots(M-J+1)]$ with the total number of cross-links $M$ in the system and the disorder average [ · ]. In Appendix B.6.1 we find that

$$
[M(M-1) \cdots(M-J+1)]=\left(\frac{\mu^{2} N}{2}\right)^{J} .
$$

Of particular importance is of course the case $J=1$ and $J=2$, from which the first two moments can be calculated. For those two cases, the above equation yields:

$$
\begin{aligned}
{[M] } & =\frac{\mu^{2} N}{2} \\
{[M(M-1)] } & =\left(\frac{\mu^{2} N}{2}\right)^{2} .
\end{aligned}
$$


Thus for any choice of $\mu^{2}$, the cross-link density is:

$$
\frac{[M]}{N}=\frac{\mu^{2}}{2}
$$

and with two particles involved in every cross-link, the parameter $\mu^{2}=2[M] / N$ is the average coordination number.

The fluctuations of the number of cross-links are calculated from Eqs. (2.62) with

$$
\begin{aligned}
\Delta M^{2}:=\left[M^{2}\right]-[M]^{2} & =[M(M-1)]+[M]-[M]^{2} \\
& =\left(\frac{\mu^{2} N}{2}\right)^{2}+\frac{\mu^{2} N}{2}-\left(\frac{\mu^{2} N}{2}\right)^{2}=\frac{\mu^{2} N}{2},
\end{aligned}
$$

and hence

$$
\Delta M=\sqrt{\left[M^{2}\right]-[M]^{2}}=\sqrt{\mu^{2} N / 2} .
$$

As one can easily see, for any finite $\mu^{2}$, the fluctuations $\Delta M /[M]$ relative to the mean number of cross-links vanish like $N^{-1 / 2}$.

In principle, all moments can be calculated very easily using the formula (2.61). Indeed, it turns out that all moments are equal to those of the Poisson distribution with parameter $\mu^{2} N / 2$. [Broderix et al., 2002, sec. 3.1] also found a convergence of the cross-link distribution towards a Poisson distribution for the randomly crosslinked particle model with a slightly different cross-link distribution, without the use of the replica technique. Instead of the parameter $\mu^{2}$, in that work the probability for a certain cross-link is solely determined by the typical cross-linking length.

\subsubsection{Gel Fraction}

In order to obtain a relation for the gel fraction $Q$, we need to find the minimum of the free energy with respect to $Q$. Therefore, we calculate

$$
\frac{\partial}{\partial Q} \frac{\partial f_{n+1}^{\mathrm{sp}}}{\partial n}=-\left(-\mu^{2} \exp \left(-\mu^{2} Q\right)+\mu^{2}-\mu^{2} Q\right) \ln N \stackrel{!}{=} 0
$$

which becomes an implicit equation for $Q$ :

$$
1-Q=\mathrm{e}^{-\mu^{2} Q}
$$

This equation is well known and has been derived in many contexts like random graph percolation, random nets and theory of epidemics [Solomonoff \& Rapoport, 1951; Landau, 1952; Erdős \& Rényi, 1960, 1961], as well as similar gelling systems [Broderix et al., 2002; Goldbart et al., 1996; Castillo et al., 1994]. 


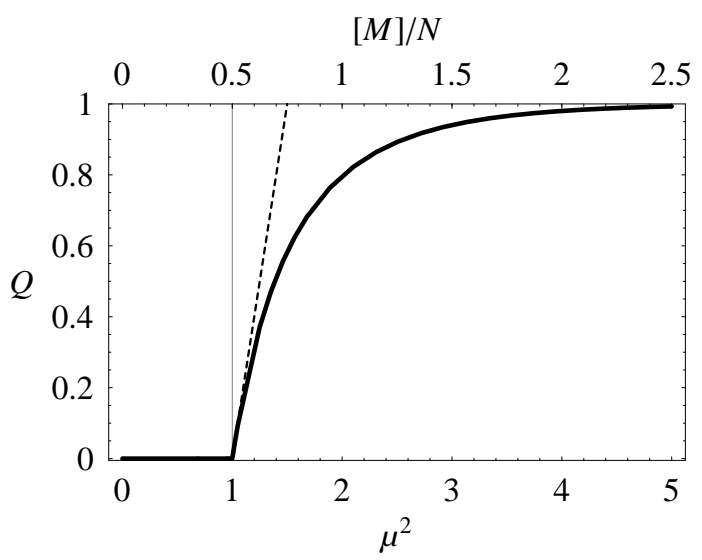

Figure 2.7: Fraction of localized particles $Q$, depending on the average coordination number. The infinite cluster forms at the critical value $\mu_{c}^{2}=1$. The full line is the exact solution of Eq. (2.67) and the dashed line the approximation (2.69).

Given that the relation for the gel fraction (2.67) is already well known, we will only briefly discuss it here: It has a trivial solution $Q=0$, which is stable for $\mu^{2} \leq 1$ and unstable for $\mu^{2}>1$. At $\mu^{2}=\mu_{c}^{2}=1$, there is a second order transition to a gel state $Q>0$, as for $\mu^{2}>1$ a further, nontrivial stable solution emerges. This is the sol-gel transition, already briefly mentioned in Sec. 2.4.5.

In Fig. 2.7, the stable branch of the gel fraction $Q$ is plotted versus the cross-link density. At a critical value $\mu_{c}^{2}=1$, one can see the transition from a sol phase, with all particles delocalized $(Q=0)$, to a gel phase, where certain particles are localized $(Q>0)$.

Close to that transition, Eq. (2.67) can be expanded for $\mu^{2} \approx 1, Q \ll 1$ [Peng et al., 1998; Goldbart et al., 2004; Mukhopadhyay et al., 2004]:

$$
\begin{aligned}
1-Q & =1-\mu^{2} Q+\frac{1}{2} \mu^{4} Q^{2}+\mathcal{O}\left(Q^{3}\right) \\
-1 & =-\mu^{2}+\frac{1}{2} \mu^{4} Q+\mathcal{O}\left(Q^{2}\right) \\
Q & =2\left(\mu^{2}-1\right)+\mathcal{O}\left(Q^{2}\right)
\end{aligned}
$$

And with $\varepsilon=\mu^{2}-1$, the distance from the critical point:

$$
Q=2 \varepsilon+\mathcal{O}\left(\varepsilon^{2}\right)
$$

In this work we are interested in the behavior for arbitrary $\mu^{2}$. In this regard, we rewrite Eq. (2.67) as:

$$
\begin{aligned}
1 & =(1-Q) \mathrm{e}^{\mu^{2} Q} \\
-\mu^{2} \mathrm{e}^{-\mu^{2}} & =\mu^{2}(Q-1) \mathrm{e}^{\mu^{2}(Q-1)} .
\end{aligned}
$$


This form can be written in terms of the Lambert function $W(x)$, which is the solution of the equation $x=W(x) \mathrm{e}^{W(x)}$, see [Corless et al., 1996], and thus we get:

$$
\begin{aligned}
\mu^{2}(Q-1) & =W\left(-\mu^{2} \mathrm{e}^{-\mu^{2}}\right) \\
Q & =1+\frac{W\left(-\mu^{2} \mathrm{e}^{-\mu^{2}}\right)}{\mu^{2}} .
\end{aligned}
$$

With the help of the expansion of the Lambert function,

$$
W(-x)=-\sum_{k=1}^{\infty} \frac{k^{k-1}}{k !} x^{k} \quad \text { for } \quad|x|<\frac{1}{\mathrm{e}}
$$

we get

$$
Q=1-\mathrm{e}^{-\mu^{2}} \sum_{k=0}^{\infty} \frac{(k+1)^{k-1}}{k !}\left(\mu^{2} \mathrm{e}^{-\mu^{2}}\right)^{k} \quad \text { for all } \mu^{2}>1
$$

\subsubsection{Shear Modulus}

In Eq. (2.60), we identified the shear modulus $G$. Restoring units of energy, it becomes:

$$
G=\left(\exp \left(-\mu^{2} Q\right)+\mu^{2} Q-1-\frac{\mu^{2} Q^{2}}{2}\right) n_{0} k_{\mathrm{B}} T
$$

As one would expect, it vanishes in the liquid phase $\left(\mu^{2}<1, Q=0\right)$. In the solid phase $\left(\mu^{2}>1\right)$, it increases linearly with particle density $n_{0}$ and depends only on the cross-link density, given the gel fraction $Q=Q\left(\mu^{2}\right)$ (derived in the previous section 2.9.2).

Close to the gel transition $\mu^{2} \gtrsim 1$, the shear modulus can be expanded for small $Q$ :

$$
\begin{aligned}
G & =\left(1-\mu^{2} Q+\frac{\left(\mu^{2} Q\right)^{2}}{2 !}-\frac{\left(\mu^{2} Q\right)^{3}}{3 !}+\mu^{2} Q-1-\frac{\mu^{2} Q^{2}}{2}\right) n_{0} k_{\mathrm{B}} T+\mathcal{O}\left(Q^{4}\right) \\
& =\left(\frac{\mu^{2}\left(\mu^{2}-1\right) Q^{2}}{2}-\frac{\mu^{6} Q^{3}}{6}\right) n_{0} k_{\mathrm{B}} T+\mathcal{O}\left(Q^{4}\right)
\end{aligned}
$$

and with Eq. (2.69), we can write the shear modulus in terms of $\varepsilon=\mu^{2}-1$, resulting in a power-law behavior (see Fig. 2.8a):

$$
G=\frac{2}{3} n_{0} k_{\mathrm{B}} T \varepsilon^{3}+\mathcal{O}\left(\varepsilon^{4}\right)
$$

In the highly cross-linked regime, we can also write the shear modulus as a power series. To this end, we start from Eq. (2.74) again and use the (exact) relation (2.67) 

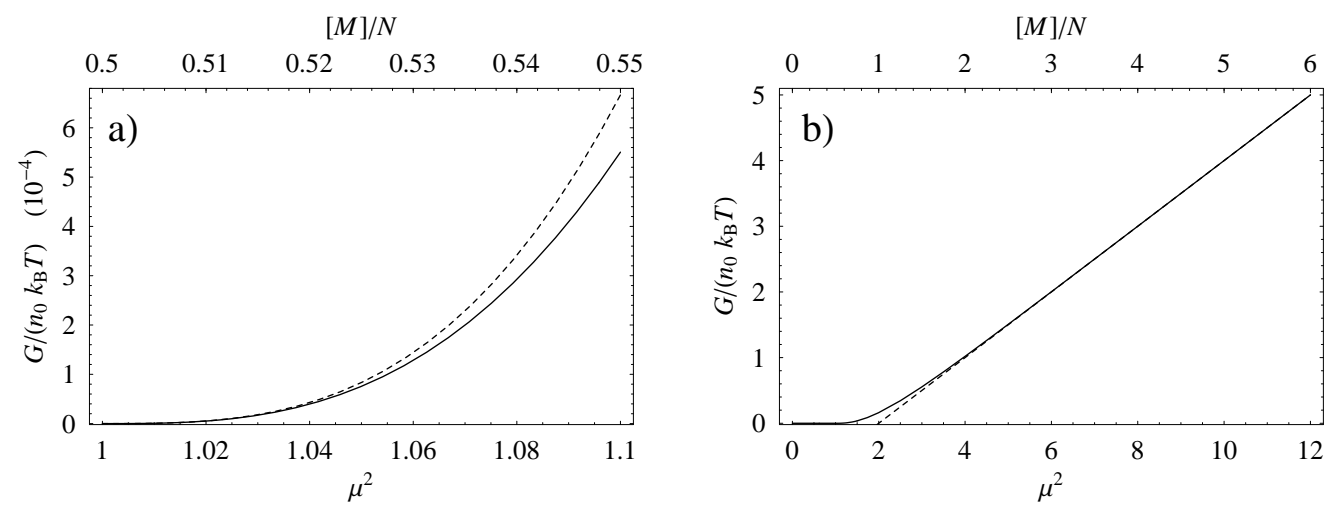

Figure 2.8: Shear modulus $G$ divided by the particle density $n_{0}$ and $k_{\mathrm{B}} T$, depending on the average coordination number $\mu^{2}$. The solid line shows the exact expression (2.74), the dashed lines are the approximations valid (a) close to the sol-gel transition, Eq. (2.76), and (b) for highly connected systems, Eq. (2.79).

to get rid of the exponential:

$$
\begin{aligned}
\frac{G}{n_{0} k_{\mathrm{B}} T} & =(1-Q)+\mu^{2} Q-1-\frac{\mu^{2} Q^{2}}{2} \\
& =\frac{\mu^{2}}{2}-1+(1-Q)-\frac{\mu^{2}}{2}(1-Q)^{2}
\end{aligned}
$$

The expansion for $(1-Q)$ is already given in Eq. (2.73). In Appendix B.6.2, we find an expression for the square of this infinite sum, $(1-Q)^{2}$, with which we easily obtain a relation for $(1-Q)-\frac{\mu^{2}}{2}(1-Q)^{2}$, see Eq. (B.118). With that, the shear modulus Eq. (2.77) can be written in a series in $\mu^{2} \mathrm{e}^{-\mu^{2}}$,

$$
\frac{G}{n_{0} k_{\mathrm{B}} T}=\frac{\mu^{2}}{2}-1+e^{-\mu^{2}} \sum_{k=0}^{\infty} \frac{(k+1)^{k-2}}{k !}\left(\mu^{2} e^{-\mu^{2}}\right)^{k},
$$

which can be used to compute $G$ for all $\mu^{2}>1$. In the highly cross-linked regime $\mu^{2} \gg 1, G$ is approximated by (see Fig. $2.8 \mathrm{~b}$ ):

$$
G=\frac{1}{2} n_{0} k_{\mathrm{B}} T\left(\mu^{2}-2\right)+\mathcal{O}\left(\mathrm{e}^{-\mu^{2}}\right)
$$

The scaling $G \sim \varepsilon^{3}$ close to the critical point has been derived previously [Castillo \& Goldbart, 1998; Mukhopadhyay et al., 2004; Goldbart et al., 2004]; the critical exponent is a result of mean field theory and expected to be modified by interacting fluctuations below $D=6$ [Peng \& Goldbart, 2000]. In the highly crosslinked limit, fluctuations are presumably weak so that our result is expected to hold even beyond the Gaussian expansion around mean field theory. The result we get is reminiscent of the classical theory of rubber elasticity, $G=n_{\mathrm{S}} k_{\mathrm{B}} T$, with the density of strands $n_{\mathrm{s}}$ being replaced by the density of cross-links: $\frac{1}{2} \mu^{2} n_{0}=[M] / V$. 
In order to compare the calculated shear modulus with experimental results, note that, if the cross-link length is small compared to the particle size, it is unlikely for one monomer in $D=3(D=2)$ dimensions to be connected to more than 12 (6) other monomers. Therefore, average coordination numbers $\mu^{2}>12(>6)$ are inaccessible in these systems. Only if long tethers can form, reaching further than the nearest neighbor, higher average coordination numbers $\mu^{2}$ are possible.

\subsection{Conclusions}

We have determined the shear modulus $G$ of a randomly cross-linked system. It only depends on the particle density $n_{0}=N / V$ and the average coordination number $\mu^{2}$. Interestingly, neither the distribution of localization lengths $\mathcal{P}\left(\xi^{2}\right)$, defined in Eq. (2.43), nor the typical cross-link length $a$ influence the macroscopic behavior of the material. This result is consistent with the observation that the shear modulus determines the response of the system to a shear deformation on the longest scales: The wavelength of the shear deformation has to be larger than all microscopic length scales. Furthermore, one observes that $G \propto k_{\mathrm{B}} T$, i.e. the shear elasticity is purely entropic. This is a consequence of our simple model with entropic springs to account for the cross-links. In more complex systems, e.g. covalently bonded random networks, one expects to find additional energetic contributions to $G$ in the highly cross-linked regime. In any case, the bulk modulus should depend on the strength of the excluded-volume interaction, and hence it is not expected to be as universal as the shear modulus.

It is also noteworthy that the shear modulus does not depend on the spatial dimension $D$, apart from the fact that the maximal coordination numbers depend on dimensionality and determine the physical range of $\mu^{2}$. Again, this could be expected, because we only consider a Gaussian expansion around the saddle point; interactions between fluctuations will most likely cause a dependence on dimensionality. 

Chapter 3

\section{Cross-Linked Directed Polymers}

\section{Contents}

3.1 Introduction $\ldots \ldots \ldots \ldots \ldots \ldots \ldots \ldots \ldots \ldots \ldots$

3.2 Properties of a Single Chain . . . . . . . . . . 33

3.2.1 Radius of Gyration of an Uncrosslinked Chain . . . . . . . 33

3.2.2 Properties of a Polymer Clamped in Space . . . . . . . . . . 34

3.3 Full Model: Cross-Linked Directed Polymers . . . . . . . . 36

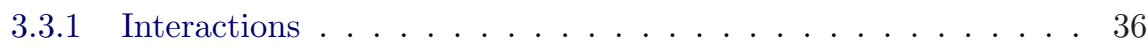

3.3 .2 Disorder Average . . . . . . . . . . . . . . . . . . . . . . 39

3.4 Replica Calculation of the Free Energy . . . . . . . . . 40

3.4.1 Disorder-Averaged Free Energy: First Steps . . . . . . . . . . 40

3.4.2 Introduction of the Replicated Density Field . . . . . . . . . 41

3.4.3 Introduction of a Field Theory and Decoupling . . . . . . . . 43

3.4.4 Saddle Point Equation . . . . . . . . . . . . . . . . . . . 44

3.5 Ansatz for the Order Parameter . . . . . . . . . . 45

3.6 The Saddle-Point Equation with Ansatz for $\Omega \ldots \ldots \ldots 47$

3.6.1 Expansion to second order in $Q \ldots \ldots \ldots$. . . . . . 48

3.6.2 Sol-Gel Transition . . . . . . . . . . . . . . . . . . . . . . 49

3.7 The Equation for the Localization Length . . . . . . . . . 49

3.7.1 Normalization of Length Scales . . . . . . . . . . . . . . 49

3.7.2 Result for the Distribution of Localization Lengths . . . . . . 50

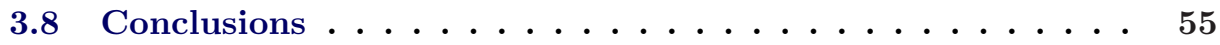

\section{$3.1 \quad$ Introduction}

In the previous chapter we investigated a system of cross-linked molecular networks. Neither the particles nor the cross-linking gave rise to a preferred direction, hence on a macroscopic scale, the system was isotropic. In many cases, however, the experimental setup induces a preferred direction of the polymer chains, like e.g. for so called polymer brushes, where strands are mounted perpendicular to a bottom plate, or DNA strands which are highly stretched along one direction. For polymer chains in a nematic solvent or nematic polymers [Kamien et al., 1992], the alignment and hence the emergence of a preferred direction can also be due 
to spontaneous symmetry breaking, whereby the chains align in an arbitrary but uniform direction, when e.g. the temperature is decreased below a critical value. Hence, the field of directed polymers has become an active area of research during the last twenty years.

This problem of directed chains is also relevant for the behavior of type-II superconductors [Kamien et al., 1992; Marchetti \& Nelson, 1993]. A magnetic field, larger than a critical value $H_{c 1}$, penetrates the superconductor in form of flux lines, which are preferably aligned along the field and each of which is carrying one quantum of magnetic flux. Due to their mutual repulsion, these flux lines can form various lattice or glassy structures in the transverse plane. In this respect, the flux lines are similar to directed polymer chains with excluded volume interaction.

Recently, there has been an increasing interest in cross-linked polymer brushes with promising applications. This is due to the fact that cross-linking can influence chemical and mechanical stability, permeability and swelling characteristics of the polymer brushes [Huang et al., 2001; Loveless et al., 2006]. For example Ionov et al. [2004] investigated binary polymer brushes, consisting of both hydrophobic and hydrophilic chains; they managed to cross-link the hydrophobic components in the extended or collapsed state, depending on the position of the sample; thereby they could locally, on a $1 \mathrm{~m}$-scale, tailor the properties of the material. On the other hand, amphiphilic block copolymers (i.e. polymer chains with one hydrophobic and one hydrophilic end) in an aqueous solution are known to form structures like micelles, lipid bilayers, or liposomes, whose interior comprises brush-like structures. For micelles Iijima et al. [1999] and Xu et al. [2004] also found that cross-linking can be used to achieve stabilization. Stable micelles are of great importance for pharmaceutical applications, since they can be utilized as carriers for targeted drug delivery.

In this chapter we investigate a simple model of flexible cross-linked directed polymers, embedded between two parallel surfaces (see Fig. 3.1). The polymer chains are modeled as functions $\mathbf{r}(z)$, which are preferably aligned along the distinguished direction $z$. This preferential alignment may be interpreted as a mechanical stretching of the chains along the $z$-direction, or as the interaction with a nematic field. We will study the effect of permanent cross-links on this simple structure and observe a gelation transition as the cross-links concentration is increased above a critical value, similar to the RLP model.

In the following section 3.2, we will discuss the behavior of one single polymer chain. The full model, comprising a large ensemble of these chains, is introduced in Sec. 3.3. In Sec. 3.4 we develop a Landau-Wilson free energy, with the replicated density being the order parameter. In Sec. 3.5 an Ansatz for the order parameter is developed, which is shown to solve the saddle-point equation in Sec. 3.6; the sol-gel transition, a consequence of the saddle-point equation, is investigated there (see Sec. 3.6.2). The distribution for the localization lengths is derived in Sec. 3.7, and we will conclude in Sec. 3.8. 


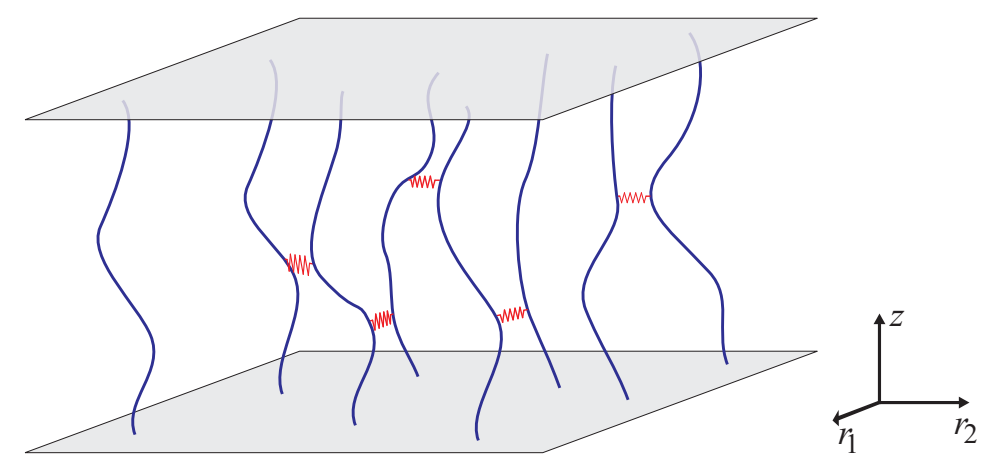

Figure 3.1: Illustration of the cross-linked directed polymer model. The polymers are depicted as blue lines ( 2 ). They are preferably aligned along the $z$-axis and are randomly cross-linked ( $\mathrm{mm})$. We will refer to the directions perpendicular to the axis of alignment as the transverse or in-plane direction (here $r_{1}$ and $r_{2}$ ).

\subsection{Properties of a Single Chain}

Before starting with the system of cross-linked directed polymers, it is illustrative to investigate the behavior of one single polymer chain. Such a chain is modeled as a $D$ dimensional vector $\mathbf{r}(z)$ that depends on an additional dimension, the system height $z$. We will have a look at fluctuations of a fully uncrosslinked chain (Sec. 3.2.1) as well as a chain clamped to a certain point in space (Sec. 3.2.2).

\subsubsection{Radius of Gyration of an Uncrosslinked Chain}

Consider a single free polymer chain $\mathbf{r}(z)$ only subject to the alignment interaction

$$
H_{\text {align }, 1}=\frac{\sigma}{2} \int_{0}^{L} \mathrm{~d} z \dot{\mathbf{r}}^{2}(z)
$$

which penalizes deviations from the alignment along the $z$-axis. Throughout this chapter about directed polymers, the index ${ }_{1}$ on any Hamiltonian specifies that it is acting only on one chain, to avoid confusion with Hamiltonians acting on the whole system of $N$ chains, investigated later. The probability for a conformation $\mathbf{r}(z)$ is given by the Boltzmann weight $\mathrm{e}^{-H_{\text {align,1, }}}$. Here we want to calculate the average extension of this chain in $\mathbf{r}$-direction, or in other words, its transverse radius of gyration:

$$
R_{\mathrm{g}}^{2}:=\left\langle\frac{1}{2 L^{2}} \int_{0}^{L} \mathrm{~d} z_{1} \mathrm{~d} z_{2}\left(\mathbf{r}\left(z_{1}\right)-\mathbf{r}\left(z_{2}\right)\right)^{2}\right\rangle_{H_{\text {align, } 1}} .
$$

The average $\langle\cdot\rangle_{H_{\text {align,1 }}}$ is represented by a functional integral over all chain conformations. For an arbitrary observable $\mathcal{A}$,

$$
\langle\mathcal{A}\rangle_{H_{\text {align }, 1}}=\int \mathcal{D} \mathbf{r}(z) \mathcal{A} \mathrm{e}^{-H_{\text {align }, 1}} .
$$


The measure $\operatorname{Dr}(z)$ of all functional integrals in this thesis is normalized such that

$$
\int \mathcal{D} \mathbf{r}(z) \mathrm{e}^{-H_{\text {align }, 1}}=1
$$

That is why Eq. (3.3) does not need a normalization. In Appendix C.1.1, we solve Eq. (3.2) by a discretization of the functional integral and get for the radius of gyration:

$$
R_{\mathrm{g}}^{2}=\frac{D}{6} \frac{L}{2 \sigma}
$$

We will see that the quantity

$$
\ell^{2}:=\frac{L}{2 \sigma} \propto R_{\mathrm{g}}^{2}
$$

will be of great importance in the full system of cross-linked polymer chains, which we investigate later.

\subsubsection{Properties of a Polymer Clamped in Space}

As a next step we calculate the properties of a single polymer chain which is clamped to a fixed point $\left(\mathbf{0}, z_{\mathrm{c}}\right)$ in space, see Fig. 3.2, left. Hence the chain $\mathbf{r}(z)$ is subject to the following two interactions $H_{1}=H_{\text {align }, 1}+H_{\text {clamp }, 1}$ with:

$$
\begin{aligned}
H_{\text {align }, 1} & =\frac{\sigma}{2} \int_{0}^{L} \mathrm{~d} z \dot{\mathbf{r}}^{2}(z) \\
H_{\text {clamp }, 1} & =\frac{1}{2 a^{2}}\left(\mathbf{r}\left(z_{\mathrm{c}}\right)-\mathbf{0}\right)^{2}
\end{aligned}
$$

To investigate properties of this simple system, we introduce the partition function with an auxiliary field $\mathbf{b}$ :

$$
Z=\int \mathcal{D} \mathbf{r}(z) \exp \left(-H_{1}+\mathbf{b} \cdot \mathbf{r}\left(z_{0}\right)\right)
$$

so that we can easily compute the mean fluctuations of the chain at height $z_{0}$ :

$$
\nabla_{\mathbf{b}}^{2} \ln Z=\nabla_{\mathbf{b}}\left(\frac{\nabla_{\mathbf{b}} Z}{Z}\right)=\frac{\nabla_{\mathbf{b}}^{2} Z}{Z}-\frac{\left(\nabla_{\mathbf{b}} Z\right)^{2}}{Z^{2}}=\left\langle\mathbf{r}^{2}\left(z_{0}\right)\right\rangle-\left\langle\mathbf{r}\left(z_{0}\right)\right\rangle^{2}=: \Delta \mathbf{r}^{2}\left(z_{0}\right) .
$$

The functional integral in Eq. (3.9) can be solved by a discretization, similar to one in the previous section. In Appendix C.1.2 we find:

$$
\ln Z=\frac{\mathbf{b}^{2}}{2}\left(\frac{\left|z_{\mathrm{c}}-z_{0}\right|}{\sigma}+a^{2}\right)+\ln \left(\frac{\left(2 \pi a^{2}\right)^{D / 2}}{A}\right) .
$$



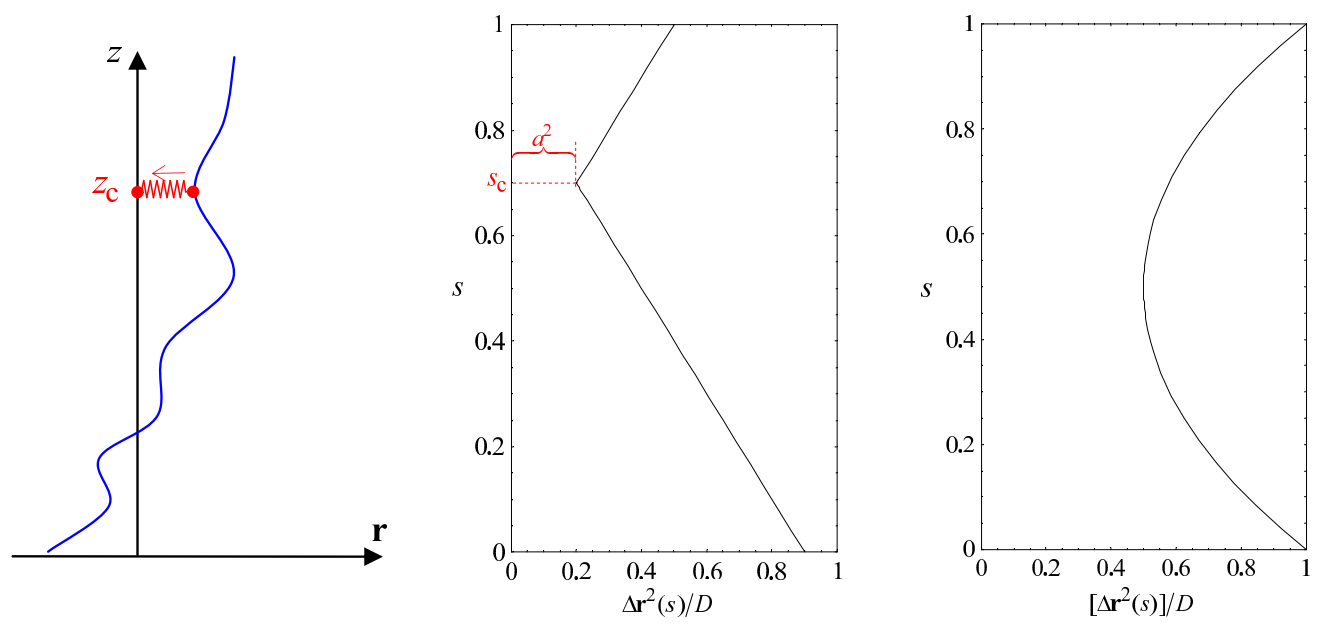

Figure 3.2: Left: Illustration of the clamped polymer chain. The polymer ( 2 ) is clamped $(\mathrm{mm})$ to the $z$-axis at a certain height $z_{\mathrm{c}}$. Center: Plot of the size of the fluctuations $\Delta \mathbf{r}^{2}(s)$, see Eq. (3.12), exemplarily for the case $a^{2}=0.2, s_{\mathrm{c}}=$ 0.7. Right: Disorder-averaged fluctuations $\left[\Delta \mathbf{r}^{2}(s)\right] \propto s^{2}+(1-s)^{2}$, according to Eq. (3.14) for the case $a^{2}=0$.

With that result we can apply Eq. (3.10) to get the fluctuations of the chain (for simplification we change $z_{0}$ to $z$ ):

$$
\Delta \mathbf{r}^{2}(z)=D \cdot\left(\frac{\left|z_{\mathrm{c}}-z\right|}{\sigma}+a^{2}\right)=D \cdot\left(\frac{L}{\sigma}\left|s_{\mathrm{c}}-s\right|+a^{2}\right) .
$$

On the right hand side of the above equation, we introduced the normalized system height $s=z / L$. This result is illustrated in Fig. 3.2, center. As one can see, the extent of the fluctuations increases linearly, as we move away from the "clamping height" $z_{\mathrm{c}}$. At the clamping height itself, $z=z_{\mathrm{c}}$, fluctuations are still present due to the finite cross-link strength $1 / a^{2}$.

In the full system, the chains will be cross-linked to each other at random heights. Similarly, we may define a disorder average [ · ] for this simple system as the uniform distribution of all clamping heights $z_{\mathrm{c}}$ (resp. $s_{\mathrm{c}}$ ), so that the disorderaveraged fluctuations may be interpreted as the average of many (non-interacting) chains clamped to random points on the $z$-axis:

$$
\begin{aligned}
{\left[\Delta \mathbf{r}^{2}(s)\right] } & :=\int_{0}^{1} \mathrm{~d} s_{\mathrm{c}} \Delta \mathbf{r}^{2}(s)=D\left(\frac{L}{\sigma} \int_{0}^{1} \mathrm{~d} s_{\mathrm{c}}\left|s_{\mathrm{c}}-s\right|+a^{2}\right) \\
& =D\left(\frac{L}{\sigma} \cdot \frac{s^{2}+(1-s)^{2}}{2}+a^{2}\right) .
\end{aligned}
$$

Using the radius of gyration $\ell^{2}=L /(2 \sigma)$ from the previous section 3.2 .1 we get:

$$
\frac{\left[\Delta \mathbf{r}^{2}(s)\right]}{D}=\ell^{2}\left(s^{2}+(1-s)^{2}\right)+a^{2}
$$




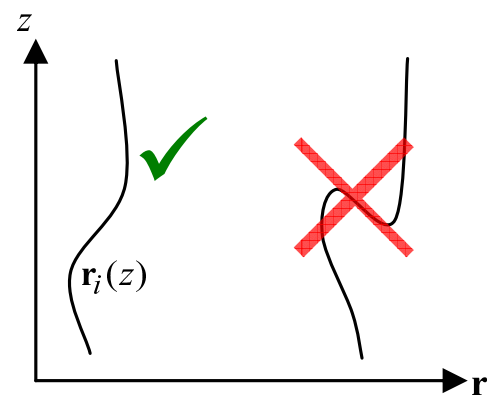

Figure 3.3: The polymer chains are modeled as functions $\mathbf{r}_{i}(z)$. Thus, at every height $z$, a chain can only appear once (as on the left) and cannot turn around in $z$ direction (as on the right).

which is illustrated in Fig. 3.2 on the right side. It might be surprising that in spite of the homogeneous distribution of clamping points, the fluctuations are not uniform along the $z$-axis. This behavior arises from the fact that the chain has open ends, which can move around freely. We will see a similar behavior also for the cross-linked polymers and discuss it more extensively in Sec. 3.7.2.

\subsection{Full Model: Cross-Linked Directed Polymers}

In the rest of this chapter, we consider a system of $N$ identical chains. As in the previous section, the positions of these chains are modeled as $D$-dimensional functions $\mathbf{r}_{1}(z), \ldots, \mathbf{r}_{N}(z)$ which depend on the "height" $z$ in the system, see Fig. 3.3, left chain. Thus, the dimensionality of the system is $D+1$. This way of modeling distinguishes the $z$-direction such that the chains cannot "turn around" and go back in that direction as the chain on the right hand side in Fig. 3.3.

The chains reside in a volume $V=L \cdot A$, where $L$ is the total height in $z$-direction and $A$ is the area (or hyper-area) of the system in the other $D$ spatial dimensions. Most important is, of course, the case $D=2$ for a three-dimensional system. The thermodynamic limit is taken such that $N, A \rightarrow \infty$ with a constant chain density $n_{0}=N / A$. However, we keep the $L$-dependence, since the free ends of the chain may cause a $z$-dependence of the structural and mechanical properties that provides interesting physics. It is interesting to note about this model that the limit $L \rightarrow 0$ (a thin $D$-dimensional "slice") is reproduces the $D$-dimensional RLP model.

\subsubsection{Interactions}

The chains are subject to the following interactions:

The cross-link interaction permanently connects a pair of chains $\left(i_{e}\right.$ and $\left.j_{e}\right)$ at a fixed height $\left(z_{e} \in[0, L]\right)$, which is the same for both constituents (see left side of Fig. 3.4). As in the randomly cross-linked particle model, the $M$ crosslinks are modeled as Hookian springs. Thus, for a given cross-link configuration 

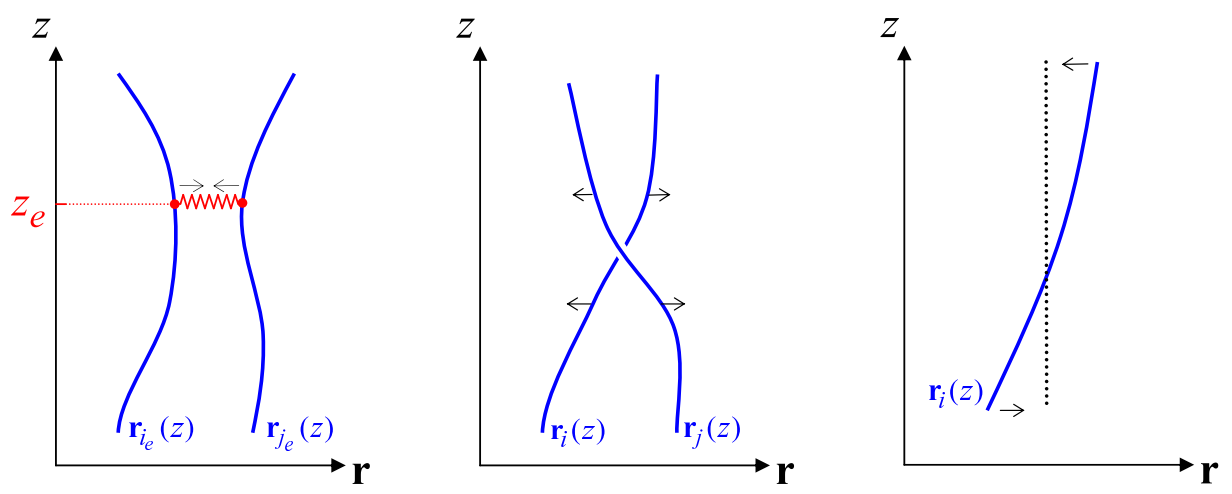

Figure 3.4: Illustration of the interactions between the directed polymers ( ( ). Left: A cross-link $(\mathrm{mm})$ permanently connects chains $i_{e}$ and $j_{e}$ at height $z_{e}$. Center: Through the excluded volume interaction, chains which come too close are repelled from each other. Right: The alignment interaction straightens the chains along the $z$ axis by penalizing high derivatives $\dot{\mathbf{r}}_{i}(z)$.

$\mathcal{C}:=\left\{\left(i_{e}, j_{e}, z_{e}\right)\right\}_{e=1}^{M}$, the cross-link Hamiltonian is:

$$
H_{\mathrm{Xlink}}=\frac{1}{2 a^{2}} \sum_{e=1}^{M}\left(\mathbf{r}_{i_{e}}\left(z_{e}\right)-\mathbf{r}_{j_{e}}\left(z_{e}\right)\right)^{2}
$$

where $a$ is the typical cross-linking length.

As in the RLP model, a repulsive excluded-volume interaction is introduced, to prevent the system from collapsing. Here, it acts between all pairs of chains and along the whole length of them (see Fig. 3.4, center):

$$
H_{\mathrm{ev}}=\frac{\lambda}{2} \sum_{i, j=1}^{N} \int_{0}^{L} \mathrm{~d} z U\left(\mathbf{r}_{i}(z)-\mathbf{r}_{j}(z)\right)
$$

with a short ranged $U(\mathbf{x})$ subject to the restriction $\int_{A} U(\mathbf{x}) \mathrm{d} \mathbf{x}=1$.

Furthermore, we add an alignment interaction, which straightens the chains along the $z$-axis by penalizing high values of the derivatives $\dot{\mathbf{r}}_{i}(z)$ (Fig. 3.4, right):

$$
H_{\text {align }}=\frac{\sigma}{2} \sum_{i=1}^{N} \int_{0}^{L} \mathrm{~d} z \dot{\mathbf{r}}_{i}^{2}(z)
$$

In Sec. 3.2, we have already seen the effect of this interaction on a single chain.

From a physical point of view, this interaction can be interpreted as a means to penalize stretching of the chains: While the unperturbed length, i.e. the length of a 


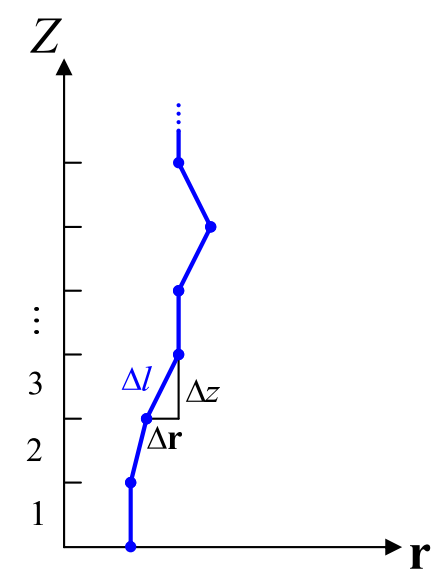

Figure 3.5: Discretization of a polymer chain into segments $Z=1,2, \ldots, Z_{L}$. The length of one chain segment $\Delta l$ depends on the length of the segment in $z$-direction $(\Delta z)$ and in transverse direction $(\Delta \mathbf{r})$.

chain with $\mathbf{r}(z) \equiv \mathbf{0}$, equals the system height $L$, any fluctuation $\mathbf{r}(z) \neq \mathbf{0}$ stretches the chain such that the $\operatorname{arc}$ length $L_{\text {arc }}$ becomes larger than $L$ : Discretizing the chain into $Z_{L}$ segments of height $\Delta z=L / Z_{L}$ (see Fig. 3.5), we get for its arc length:

$$
\begin{array}{r}
L_{\mathrm{arc}}=\sum_{Z=1}^{Z_{L}} \Delta l_{Z}=\sum_{Z=1}^{Z_{L}} \sqrt{\Delta z^{2}+\Delta \mathbf{r}_{Z}^{2}}=\sum_{Z=1}^{Z_{L}} \Delta z \sqrt{1+\frac{\Delta \mathbf{r}_{Z}^{2}}{\Delta z^{2}}} \\
\stackrel{\Delta z \rightarrow 0}{=} \int_{0}^{L} \sqrt{1+\dot{\mathbf{r}}^{2}(z)} \mathrm{d} z=L+\frac{1}{2} \int_{0}^{L} \dot{\mathbf{r}}^{2}(z) \mathrm{d} z+\mathcal{O}\left(\dot{\mathbf{r}}^{4}\right) .
\end{array}
$$

Thus, to lowest order in $\dot{\mathbf{r}}(z)$, the alignment Hamiltonian (3.17) comprises the excess length $L_{\mathrm{arc}}-L=\frac{1}{2} \int_{0}^{L} \dot{\mathbf{r}}^{2}(z) \mathrm{d} z$, of all chains. This discretization of a chain into small segments $Z=1,2, \ldots, Z_{L}$ will appear frequently throughout the investigation of this model and is a useful way to visualize and calculate functional integrals.

A more sophisticated approach [Marko \& Siggia, 1995] shows that this Hamiltonian is also obtained for a worm-like chain (a model of semiflexible and inextensible chains), if it is strongly stretched along the $z$-axis by applying an external force $f$ to the ends of the chain. With our notation the energy of this stretched worm-like chain can be written as $E=\frac{1}{2} \int_{0}^{L_{\text {arc }}} \mathrm{d} l\left(A \ddot{\mathbf{r}}^{2}(l)+f \dot{\mathbf{r}}^{2}(l)\right)$, where $A$ is the persistence length. If the force on the chain ends $f$ is strong enough, the bending contribution can be neglected. Changing the integral over the arc length $\int_{0}^{L_{\text {arc }}} \mathrm{d} l$ to an integral over the stretching direction $\int_{0}^{L} \mathrm{~d} z$ we get a transformation factor $\mathrm{d} l / \mathrm{d} z=\sqrt{1+\dot{\mathbf{r}}^{2}(z)}=1+\mathcal{O}\left(\dot{\mathbf{r}}^{2}\right)$. Thus this strongly stretched worm-like chain reproduces to lowest order the alignment Hamiltonian (3.17) with $\sigma$ playing the role of $f$.

Another physical realization of Eq. (3.17) is that of worm-like chains interacting with a strong nematic field, in the limit of vanishing persistence length of the chains [Kamien et al., 1992]. In this context, $\sigma$ controls the coupling of a 
(homogeneous) nematic matrix to the local polymer direction. Different from the interpretation of stretched chains, polymers in a nematic field would in general be allowed to make hairpin turns, since the nematic field is typically symmetric under flipping the direction of the director field $\mathbf{n} \rightarrow-\mathbf{n}$; the energy of a hairpin is estimated to be $E_{h} \approx \sqrt{\sigma \kappa}$ (with the bending stiffness $\kappa$ ) [de Gennes, 1982; Nelson, 2002]. In this context it is important to note that our model is an approximation, since such hairpins cannot be modeled by a function $\mathbf{r}(z)$. However, since we are interested in the limit in which coupling strength $\sigma$ is dominating further terms, hairpins are energetically unfavorable and can savely be neglected if $\sigma \gg \kappa^{-1}$ is ensured.

The alignment interaction (3.17) is also necessary from a mathematical point of view, because there is no other interaction that makes sure that the chains are continuous in $z$-direction. Without the alignment interaction, every slice in $z$-direction could be treated independently and system would decouple.

The alignment interaction has the convenient form of a sum over all individual chains,

$$
H_{\text {align }}=\sum_{j=1}^{N} H_{\text {align }, 1}\left\{\mathbf{r}_{j}\right\},
$$

which, as we will see, is easy to handle in the calculation. It is even possible, without impeding the calculation, to add further interactions of that form. Beyond the alignment interaction, further possible terms proposed in Appendix C.2.

The total Hamiltonian is $H_{\mathcal{C}}=H_{\mathrm{Xlink}}+H_{\mathrm{ev}}+H_{\mathrm{align}}$. For the partition function, we have to use functional integrals over all possible conformations of all chains

$$
Z_{\mathcal{C}}=\int \mathcal{D} \mathbf{r}_{1}(z) \cdots \mathcal{D} \mathbf{r}_{N}(z) \mathrm{e}^{-H_{\mathcal{C}}}
$$

The functional integral $\int \mathcal{D} \mathbf{r}(z)$ was already briefly mentioned in Sec. 3.2. For a quantity $\mathcal{A}(\{\mathbf{r}(z)\})$ that depends on the chain conformation, it is represented by a discretization as in Fig. 3.5:

$$
\int \mathcal{D} \mathbf{r}(z) \mathcal{A}(\{\mathbf{r}(z)\})=\frac{\int \mathrm{d} \mathbf{r}_{1} \cdots \mathrm{d} \mathbf{r}_{Z_{L}} \mathcal{A}\left(\left\{\mathbf{r}_{Z}\right\}_{Z=1}^{Z_{L}}\right)}{\int \mathrm{d} \mathbf{r}_{1} \cdots \mathrm{d} \mathbf{r}_{Z_{L}} \exp \left(-\frac{\sigma}{2} \sum_{Z=1}^{Z_{L}-1} \frac{\left(\mathbf{r}_{Z+1}-\mathbf{r}_{Z}\right)^{2}}{\Delta z^{2}}\right)}
$$

The normalization constant in the denominator prevents divergences for $Z_{L} \rightarrow \infty$ and is such that $\int \mathcal{D} \mathbf{r}(z) \mathrm{e}^{-H_{\text {align }, 1}\{\mathbf{r}(z)\}}=1$.

\subsubsection{Disorder Average}

As in the RLP model, we use the Deam-Edwards distribution as cross-link distribution (see Sec. 2.3). In addition to taking into account all possible cross-links by 
summing over all possible pairs of chains, we also have to integrate over all possible cross-link heights $z_{e}$ :

$$
\begin{gathered}
{\left[\mathcal{A}_{\mathcal{C}}\right]=\sum_{M=0}^{\infty} \sum_{\substack{i_{1}, \ldots, i_{M}, j_{1}, \ldots, j_{M}=1}}^{N} \frac{1}{L^{M}} \int_{0}^{L} \mathrm{~d} z_{1} \cdots \mathrm{d} z_{M} \mathcal{A}_{\mathcal{C}} \cdot P_{\mathrm{DE}}(\mathcal{C})} \\
\text { with } \quad P_{\mathrm{DE}}(\mathcal{C})=\frac{1}{\mathcal{Z}_{1}} \cdot \frac{\left(\tilde{\mu}^{2}\right)^{M}}{M !} Z_{\mathcal{C}} \\
\text { where } \quad \tilde{\mu}^{2}=\frac{\mu^{2}}{2 N \phi} \text { and } \phi=\frac{\left(2 \pi a^{2}\right)^{D / 2}}{A}
\end{gathered}
$$

And the normalization constant $\mathcal{Z}_{1}$ is such that $[1]=1$.

The average cross-link density is given by $[M] / N=\mu^{2} / 2$, as in the RLP model; the calculation of $[M]$ is deferred to Appendix C.7, as it is very similar to the RLP model (Secs. 2.9.1 and B.6.1).

\subsection{Replica Calculation of the Free Energy}

\subsubsection{Disorder-Averaged Free Energy: First Steps}

We are interested in the disorder-averaged free energy and, again, use the replica trick to handle the average over the logarithm:

$$
\left[F_{\mathcal{C}}\right]=-\left[\ln Z_{\mathcal{C}}\right]=-\left[\lim _{n \rightarrow 0} \frac{Z_{\mathcal{C}}^{n}-1}{n}\right]=-\lim _{n \rightarrow 0} \frac{\left[Z_{\mathcal{C}}^{n}\right]-1}{n} .
$$

The disorder-averaged $Z_{\mathcal{C}}^{n}$ is calculated by applying Eq. (3.22), which leads to the introduction of $n+1$ replicas:

$$
\begin{aligned}
& {\left[Z_{\mathcal{C}}^{n}\right]=\frac{1}{\mathcal{Z}_{1}} \sum_{M=0}^{\infty} \frac{\left(\tilde{\mu}^{2}\right)^{M}}{M !} \sum_{\substack{i_{1}, \ldots, i_{M}, j_{1}, \ldots, j_{M}=1}}^{N} \frac{1}{L^{M}} \int_{0}^{L} \mathrm{~d} z_{1} \cdots \mathrm{d} z_{M} Z_{\mathcal{C}} \cdot Z_{\mathcal{C}}^{n}} \\
& =\frac{1}{\mathcal{Z}_{1}} \sum_{M=0}^{\infty} \frac{\left(\tilde{\mu}^{2} / L\right)^{M}}{M !} \sum_{\substack{i_{1}, \ldots, i_{M}, j_{1}, \ldots, j_{M}=1}}^{N} \int_{0}^{L} \mathrm{~d} z_{1} \cdots \mathrm{d} z_{M} \\
& \left(\int \mathcal{D} \mathbf{r}_{1}(z) \cdots \mathcal{D} \mathbf{r}_{N}(z) \mathrm{e}^{-H_{\mathrm{ev}}\left\{\mathbf{r}_{j}\right\}-H_{\text {align }}\left\{\mathbf{r}_{j}\right\}} \exp \left(-\sum_{e=1}^{M} \frac{\left(\mathbf{r}_{i_{e}}\left(z_{e}\right)-\mathbf{r}_{j_{e}}\left(z_{e}\right)\right)^{2}}{2 a^{2}}\right)\right)^{n+1} \\
& =\frac{1}{\mathcal{Z}_{1}} \sum_{M=0}^{\infty} \frac{\left(\tilde{\mu}^{2} / L\right)^{M}}{M !} \sum_{\substack{i_{1}, \ldots, i_{M}, j_{1}, \ldots, j_{M}=1}}^{N} \int_{0}^{L} \mathrm{~d} z_{1} \cdots \mathrm{d} z_{M} \\
& \int \mathcal{D} \hat{r}_{1}(z) \cdots \mathcal{D} \hat{r}_{N}(z) \mathrm{e}^{-H_{\mathrm{ev}}^{(n+1)}\left\{\hat{r}_{j}\right\}-H_{\mathrm{align}}^{(n+1)}\left\{\hat{r}_{j}\right\}} \prod_{e=1}^{M} \exp \left(-\frac{\left(\hat{r}_{i_{e}}\left(z_{e}\right)-\hat{r}_{j_{e}}\left(z_{e}\right)\right)^{2}}{2 a^{2}}\right) .
\end{aligned}
$$


Here we implemented the $(n+1)$-times replicated hatted vectors $\hat{r}_{j}(z)=$ $\left(\mathbf{r}_{j}^{(0)}(z), \ldots, \mathbf{r}_{j}^{(n)}(z)\right)$, which is the "launch" of the replica theory. Different from the calculation of the RLP model, these vectors are functions of $z$. The replicated Hamiltonians are:

$$
\begin{aligned}
& H_{\mathrm{ev}}^{(n+1)}\left\{\hat{r}_{j}\right\}=\sum_{\alpha=0}^{n} H_{\mathrm{ev}}\left\{\mathbf{r}_{j}^{(\alpha)}\right\} \\
& H_{\text {align }}^{(n+1)}\left\{\hat{r}_{j}\right\}=\sum_{\alpha=0}^{n} H_{\text {align }}\left\{\mathbf{r}_{j}^{(\alpha)}\right\}
\end{aligned}
$$

The sum over $M$ in Eq. (3.24) can be performed, as shown in Appendix C.3.1. We obtain a result similar to Eqs. (2.14) for the RLP model:

$$
\begin{aligned}
{\left[Z_{\mathcal{C}}^{n}\right] } & =\frac{\mathcal{Z}_{n+1}}{\mathcal{Z}_{1}}, \\
\text { with } \quad \mathcal{Z}_{n+1} & =\int\left(\prod_{j=1}^{N} \mathcal{D} \hat{r}_{j}(z)\right) \exp \left(-N \tilde{f}_{n+1}\left\{\hat{r}_{j}\right\}\right) \\
\text { and } \quad \tilde{f}_{n+1}\left\{\hat{r}_{j}\right\} & =-\frac{\tilde{\mu}^{2}}{L N} \sum_{i, j=1}^{N} \int_{0}^{L} \mathrm{~d} z \exp \left(-\frac{\left(\hat{r}_{i}(z)-\hat{r}_{j}(z)\right)^{2}}{2 a^{2}}\right)+\frac{H_{\mathrm{ev}}^{(n+1)}}{N}+\frac{H_{\text {align }}^{(n+1)}}{N}
\end{aligned}
$$

where $\mathcal{Z}_{n+1}$ is the replica partition function and $\tilde{f}_{n+1}\left\{\hat{r}_{j}\right\}$ is the replica free energy. At this point, it is interesting to note that, while the chains $\hat{r}_{j}(z)$ are $n+1$ replicated vectors, $z$ is not replicated. This is because the integration over $z$ was originally part of the disorder average (like the sum $\sum_{i, j}$ ), see Eq. (3.24), while the integration over the chain positions $\int \mathcal{D} \hat{r}_{j}(z)$ comes from the (replicated) partition function.

As in Eq. (2.57) for the RLP model, $\mathcal{Z}_{n+1}$ is related to the disorder-averaged free energy by:

$$
-\left[F_{\mathcal{C}}\right]=\left[\ln Z_{\mathcal{C}}\right]=\lim _{n \rightarrow 0} \frac{\partial}{\partial n} \ln \mathcal{Z}_{n+1}
$$

\subsubsection{Introduction of the Replicated Density Field}

As for the RLP model, it is useful to define a replicated density field, which can characterize the state of the system and easily distinguish between sol and gel phase. Now, it additionally depends on the height $z$ :

$$
\begin{aligned}
\text { real space: } \mathrm{O}(\hat{x}, z):=\frac{1}{N} \sum_{j=1}^{N} \delta\left(\hat{x}-\hat{r}_{j}(z)\right), \\
\text { Fourier space: } \mathrm{O}(\hat{q}, z):=\frac{1}{N} \sum_{j=1}^{N} \exp \left(\mathrm{i} \hat{q} \hat{r}_{j}(z)\right) .
\end{aligned}
$$


For a slice at a given system height $z$, the replicated density $\mathrm{O}(\hat{x}, z)$ gives the probability to find a particle at $\mathbf{x}^{(0)}$ in replica (0), at $\mathbf{x}^{(1)}$ in replica $(1), \ldots$ and at $\mathbf{x}^{(n)}$ in replica $(n)$. Hence it can answer the critical question, whether $\left|\mathbf{r}_{j}^{(\alpha)}(z)-\mathbf{r}_{j}^{(\beta)}(z)\right|$ remains finite for different replicas $\alpha$ and $\beta$, i.e. whether or not the chain segment of polymer $j$ at height $z$ is localized; and if so, it can give information about the extent $\xi$ of the localization.

This localization of the polymer chains occurs when the cross-link structure gives rise to a macroscopic cluster: if a chain is part of that macroscopic cluster, it will be hindered from traversing the whole system ${ }^{1}$; hence the replicated density (3.29) is capable of detecting the emergence of a macroscopic cluster and therefore distinguish between sol and gel phase.

As for the RLP model, we distinguish between the one replica sector (1RS), the set of $\hat{q}$-vectors with exactly one of the $n+1$ replica components non-zero, and the higher replica sector (HRS), the set of $\hat{q}$-vectors with at least two non-zero components; see Sec. 2.4.3. For $\hat{q} \in 1 \mathrm{RS}$, Eq. (3.29b) is the simple Fourier density of the respective replica, and therefore describes density fluctuations at height $z$ :

$$
\mathrm{O}\left(\left(\mathbf{0}, \ldots, \mathbf{0}, \mathbf{q}^{(\alpha)}, \mathbf{0}, \ldots, \mathbf{0}\right), z\right)=\frac{1}{N} \sum_{j=1}^{N} \exp \left(\mathrm{iq}^{(\alpha)} \mathbf{r}_{j}^{(\alpha)}(z)\right) .
$$

In Appendix C.3.2, we see how we can rewrite the replica free energy (3.27c) in terms of the density field (3.29b):

$$
\begin{aligned}
\tilde{f}_{n+1}\left\{\hat{r}_{j}\right\}= & -\phi^{n} \frac{\mu^{2}}{2 L} \int_{0}^{L} \mathrm{~d} z \sum_{\hat{q} \in \mathrm{HRS}}|\mathrm{O}(\hat{q}, z)|^{2} \Delta(\hat{q})+\frac{1}{2} \int_{0}^{L} \mathrm{~d} z \sum_{\hat{q} \in 1 \mathrm{RS}}|\mathrm{O}(\hat{q}, z)|^{2} \tilde{\lambda}(\hat{q}) \\
& +f_{0}+\frac{H_{\text {align }}^{(n+1)}\left\{\hat{r}_{j}\right\}}{N}
\end{aligned}
$$

where we defined the mean chain density $n_{0}=N / A$ as the number of chains per (hyper-)area and:

$$
\begin{aligned}
f_{0} & =-\phi^{n} \frac{\mu^{2}}{2}+(n+1) \frac{\lambda n_{0} L}{2}, \\
\Delta(\hat{q}) & =\exp \left(-\frac{\hat{q}^{2} a^{2}}{2}\right) \\
\tilde{\lambda}(\hat{q}) & =\lambda n_{0} U(\hat{q})-\phi^{n} \frac{\mu^{2}}{L} \Delta(\hat{q}) .
\end{aligned}
$$

As we can see in Eq. (3.31a), $\tilde{\lambda}(\hat{q})$ controls the fluctuations of the unreplicated density in the 1RS, and as in the RLP model, it has to be positive to make sure the

\footnotetext{
${ }^{1}$ If chain $i_{e}$ is clamped to the macroscopic cluster at height $z_{e}$, one might ask the question, if its chain segment at a height $z_{0}$ far from the "clamping point" is still localized. However, as long as the transverse radius of gyration of the chain is much less than the transverse extent of the system, i.e. $\ell=\sqrt{L /(2 \sigma)} \ll A^{1 / D}\left({ }^{*}\right)$, a chain localized at height $z_{e}$ should be localized at any other height $z_{0}$. If the thermodynamic limit is taken such that $L \propto A^{1 / D}$ or $L<A^{1 / D},\left(^{*}\right)$ is fulfilled.
} 
system does not collapse. In Eq. (3.31d) it is interesting to see that the strength of the excluded volume interaction is counteracted by the cross-link density per height, $\mu^{2} / L$, not by the cross-link density $\mu^{2}$ alone as in the RLP model. That means that the cross-link density $\mu^{2}$ has to scale linearly with the system height $L$, if we want to keep the system properties the same, in particular to compensate a fixed excluded volume interaction. This makes sense intuitively, since the excluded volume interaction acts on the polymers along the whole height of the system, see Eq. (3.16), whereas the cross-link interaction only acts at the given points $z_{e}$, see Eq. (3.15).

\subsubsection{Introduction of a Field Theory and Decoupling}

The expression (3.31a) has the disadvantage that the density $\mathrm{O}(\hat{q}, z)$ in the upper line appears in a quadratic form. As we did for the RLP model (in Sec. 2.4.4/Appendix B.2), we want to linearize those two terms with the help of the HubbardStratonovich transformation. Since $\mathrm{O}(\hat{q}, z) \propto \sum_{j} \mathrm{e}^{\mathrm{i} \hat{q} \hat{r}_{j}(z)}$, this has the great advantage that those two terms can be written as a single sum over all particles; then in the partition function $(3.27 \mathrm{~b})$ the integral $\int \mathcal{D} \hat{r}_{1}(z) \cdots \mathcal{D} \hat{r}_{N}(z)$ decouples with respect to the particles $j=1, \ldots, N$, which drastically simplifies the calculation. The drawback of the linearization is that we have to introduce the integration over a new field $\Omega(\hat{q}, z)$, whose expectation value, however, is the same as $\mathrm{O}(\hat{q}, z)$.

As we see in Appendix C.3.3, we obtain for the replica partition function:

$$
\mathcal{Z}_{n+1}=\exp \left(-N f_{0}\right) \int \mathcal{D} \Omega \exp \left(-N f_{n+1}\{\Omega\}\right)
$$

with a replica free energy, dependent on the field $\Omega(\hat{q}, z)$ :

$$
f_{n+1}\{\Omega\}=\phi^{n} \frac{\mu^{2}}{2 L} \int_{0}^{L} \mathrm{~d} z \sum_{\hat{q} \in \mathrm{HRS}}|\Omega(\hat{q}, z)|^{2} \Delta(\hat{q})+\frac{1}{2} \int_{0}^{L} \mathrm{~d} z \sum_{\hat{q} \in 1 \mathrm{RS}}|\Omega(\hat{q}, z)|^{2} \tilde{\lambda}(\hat{q})-\ln \mathfrak{z},
$$

with an effective one-particle partition function:

$$
\begin{aligned}
\mathfrak{z}=\int \mathcal{D} \hat{r}(z) \exp \left(\phi^{n} \frac{\mu^{2}}{L} \int_{0}^{L} \mathrm{~d} z \sum_{\hat{q} \in \mathrm{HRS}} \Delta(\hat{q}) \Omega(\hat{q}, z) \mathrm{e}^{-\mathrm{i} \hat{q} \hat{r}(z)}\right. \\
\left.\quad+\mathrm{i} \int_{0}^{L} \mathrm{~d} z \sum_{\hat{q} \in 1 \mathrm{RS}} \tilde{\lambda}(\hat{q}) \Omega(\hat{q}, z) \mathrm{e}^{-\mathrm{i} \hat{q} \hat{r}(z)}-H_{\text {align, } 1}^{(n+1)}\{\hat{r}(z)\}\right)
\end{aligned}
$$

and the constant contribution $f_{0}$ as before (see Eq. (3.31b)).

This form can be used as a starting point for answering many questions arising from this system, like the density-density correlation function, the response of the system to a tilt field, the dependence of the bulk modulus on $\lambda$, the shear modulus, etc. It is important to note that no approximation has been made so far.

In this thesis, we will focus on the gelation transition of this system and the localization length of the chains. We take the limit of infinitely strong excluded 
volume interaction by taking the limit $\lambda \rightarrow \infty$ and hence $\tilde{\lambda}(\hat{q}) \rightarrow \infty$. This prevents fluctuations of the density in the one replica sector and simplifies the equation:

$$
\begin{aligned}
& f_{n+1}\{\Omega\}=\phi^{n} \frac{\mu^{2}}{2 L} \int_{0}^{L} \mathrm{~d} z \sum_{\hat{q} \in \mathrm{HRS}}|\Omega(\hat{q}, z)|^{2} \Delta(\hat{q})-\ln \mathfrak{z}, \\
& \mathfrak{z}=\int \mathcal{D} \hat{r}(z) \exp \left(\phi^{n} \frac{\mu^{2}}{L} \int_{0}^{L} \mathrm{~d} z \sum_{\hat{q} \in \mathrm{HRS}} \Delta(\hat{q}) \Omega(\hat{q}, z) \mathrm{e}^{-\mathrm{i} \hat{q} \hat{r}(z)}-H_{\text {align, } 1}^{(n+1)}\{\hat{r}(z)\}\right)
\end{aligned}
$$

\subsubsection{Saddle Point Equation}

In the RLP model, we plugged in an Anstaz for the order parameter into the replica free energy, in order to calculate quantities like the energy cost of shear deformations. Here, we are interested in the localization of the chains. Therefore, we set up the saddle point equation for the order parameter and plug in a physically motivated Ansatz similar to the one employed in Sec. 2.6. We will see that the Ansatz consistently solves the saddle point equation, yielding an equation for the distribution of localization lengths.

The saddle point equation can be retrieved from deriving $f_{n+1}\{\Omega\}$, Eq. (3.33), with respect to $\Omega:^{2}$

$$
0 \stackrel{!}{=} \frac{\delta f_{n+1}\{\Omega\}}{\delta \Omega\left(\hat{q}_{0}, z_{0}\right)}=\phi^{n} \frac{\mu^{2}}{L} \Omega\left(-\hat{q}_{0}, z_{0}\right) \Delta\left(\hat{q}_{0}\right)-\left\langle\phi^{n} \frac{\mu^{2}}{L} \Delta\left(\hat{q}_{0}\right) \mathrm{e}^{-\mathrm{i} \hat{q}_{0} \hat{r}\left(z_{0}\right)}\right\rangle_{\mathfrak{z}},
$$

which simplifies to a very plausible form:

$$
\Omega\left(-\hat{q}_{0}, z_{0}\right)=\left\langle\mathrm{e}^{-\mathrm{i} \hat{q}_{0} \hat{r}\left(z_{0}\right)}\right\rangle_{\mathfrak{z}}=: \frac{I\left(\hat{q}_{0}, z_{0}\right)}{I(\hat{0}, 0)}
$$

Here \langle\rangle$_{\mathfrak{z}}$ represents averaging with the statistical weight of the one-particle partition function $\mathfrak{z}$ (see Eq. (3.33)), and $I\left(\hat{q}_{0}, z_{0}\right)$ is defined accordingly:

$$
\begin{aligned}
& I\left(\hat{q}_{0}, z_{0}\right)= \\
& \int \mathcal{D} \hat{r}(z) \mathrm{e}^{-\mathrm{i} \hat{q}_{0} \hat{r}\left(z_{0}\right)} \exp \left(\phi^{n} \frac{\mu^{2}}{L} \int_{0}^{L} \mathrm{~d} z \sum_{\hat{q} \in \mathrm{HRS}}^{\prime} \Delta(\hat{q}) \Omega(\hat{q}, z) \mathrm{e}^{-\mathrm{i} \hat{q} \hat{r}(z)}-H_{\text {align, } 1}^{(n+1)}\{\hat{r}(z)\}\right) .
\end{aligned}
$$

\footnotetext{
${ }^{2}$ Strictly speaking, one has to take into account that $\Omega$ is complex and take both derivatives $\delta f_{n+1}\{\Omega\} / \delta \operatorname{Re} \Omega=\delta f_{n+1}\{\Omega\} / \delta \operatorname{Im} \Omega=0$. By setting $\Omega(\hat{q}, z)=\operatorname{Re} \Omega(\hat{q}, z)+\mathrm{i} \operatorname{Im} \Omega(\hat{q}, z)$ in Eq. (3.33), one can easily see that this yields the same result.
} 
The primed sum is defined by:

$$
\sum_{\hat{q} \in \mathrm{HRS}}^{\prime}(\ldots):=Q+\sum_{\hat{q} \in \mathrm{HRS}}(\ldots)
$$

The additional $Q$ seems unnecessary, since it does not depend on $\hat{r}(z)$ and hence cancels out in numerator and denominator of Eq. (3.35); however it will simplify further calculations.

As next step, we want to develop an Ansatz for the order parameter $\Omega(\hat{q}, z)$ and then show that it solves the saddle-point equation (3.35).

\subsection{Ansatz for the Order Parameter}

Here we try to develop an Ansatz for the order parameter

$$
\langle\Omega(\hat{q}, z)\rangle_{f_{n+1}}=\frac{1}{N}\left\langle\sum_{j=1}^{N} \exp \left(\mathrm{i} \hat{q} \hat{r}_{j}(z)\right)\right\rangle_{f_{n+1}}
$$

The assumptions we make are similar to the assumptions that Castillo et al. [1994] made to solve the saddle-point equations for an isotropic system, which were already mentioned in Sec. 2.6. For our system, the assumptions are:

1. A fraction $Q$ of chains is localized ("loc."). If chain $j$ is part of that fraction, its chain segment $\mathbf{r}_{j}^{(\alpha)}(z)$ at height $z$ in replica $\alpha$ performs Gaussian fluctuations around a fixed mean position $\mathbf{R}_{j}(z)$, which is independent of the replica (see Fig. 3.6). The width of this distribution is the localization length $\xi_{j}$, which follows a distribution $\mathcal{P}\left(\xi^{2}, z\right)$ that may depend on the height $z$.

2. To account for the disorder average, the mean positions $\mathbf{R}_{j}(z)$ are randomly placed with equal probability throughout the system.

3. The remaining fraction $1-Q$ is delocalized ("deloc.") and therefore has equal probability to be found anywhere in the system.

It is important to note that these assumptions will not give rise to correlations in $z$-direction. This is sufficient for our case, because we only need these assumptions for calculating the expectation value $\langle\Omega(\hat{q}, z)\rangle$, for which every slice is treated independently. If, however, we needed correlators like $\left\langle\Omega(\hat{q}, z) \Omega\left(-\hat{q}, z^{\prime}\right)\right\rangle \propto$ $\sum_{i, j=1}^{N}\left\langle\exp \left(\mathrm{i} \hat{q}\left(\hat{r}_{i}(z)-\hat{r}_{j}\left(z^{\prime}\right)\right)\right)\right\rangle$, we would need to include further assumptions, e.g. the joint probability distribution for mean positions $\mathbf{R}_{j}(z)$ and $\mathbf{R}_{j}\left(z^{\prime}\right)$ with at different heights $z$ and $z^{\prime}$.

To set up the Ansatz for the order parameter, we first incorporate the Gaussian fluctuations of a chain segment around a mean position: The joint probability density to find chain segment $j$ at position $\mathbf{x}^{(0)}$ in replica 0 , at $\mathbf{x}^{(1)}$ in replica 1 , etc. 


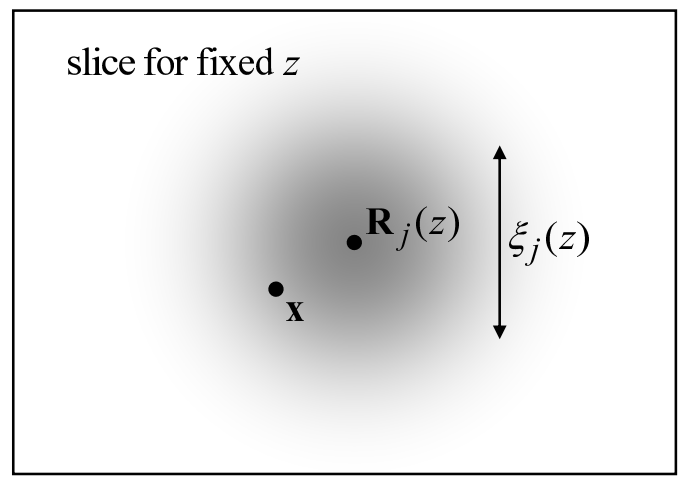

Figure 3.6: Transverse cut through the system, showing one polymer chain $j$. The probability distribution that the chain segment is at position $\mathbf{x}$ (depicted as grayscale values) is a Gaussian distribution of width $\xi_{j}(z)$ and mean position $\mathbf{R}_{j}(z)$.

is

$$
\left\langle\delta\left(\hat{x}-\hat{r}_{j}(z)\right)\right\rangle= \begin{cases}\frac{1}{\left(2 \pi \xi_{j}^{2}(z)\right)^{D(n+1) / 2}} \exp \left(-\frac{\left(\hat{x}-\hat{R}_{j}(z)\right)^{2}}{2 \xi_{j}^{2}(z)}\right) & \text { if } j \text { loc. } \\ A^{-(n+1)} & \text { if } j \text { deloc. }\end{cases}
$$

where the mean position is the same in all replicas: $\hat{R}_{j}=\left(\mathbf{R}_{j}, \ldots, \mathbf{R}_{j}\right)$. Its Fourier transform is given by:

$$
\begin{aligned}
& \left\langle\exp \left(\mathrm{i} \hat{q} \hat{r}_{j}(z)\right)\right\rangle= \\
& \quad=\int \mathrm{d} \hat{x} \exp (\mathrm{i} \hat{q} \hat{x})\left\langle\delta\left(\hat{x}-\hat{r}_{j}(z)\right)\right\rangle= \begin{cases}\exp \left(-\frac{\hat{q}^{2} \xi_{j}^{2}(z)}{2}+\mathrm{i} \hat{q} \hat{R}_{j}(z)\right) & \text { if } j \text { loc. } \\
\delta_{\hat{q}, \hat{0}} & \text { if } j \text { deloc. }\end{cases}
\end{aligned}
$$

Hence, we get for the order parameter:

$$
\begin{aligned}
\langle\Omega(\hat{q}, z)\rangle & =\frac{1}{N} \sum_{j=1}^{N}\left\langle\exp \left(\mathrm{i} \hat{q} \hat{r}_{j}(z)\right)\right\rangle \\
& =\frac{1}{N} \sum_{j \text { loc. }} \exp \left(-\frac{\hat{q}^{2} \xi_{j}^{2}(z)}{2}+\mathrm{i} \hat{q} \hat{R}_{j}(z)\right)+\frac{1}{X} \cdot X(1-Q) \delta_{\hat{q}, \hat{0}}
\end{aligned}
$$

Now, to account for the disorder average, we assume an even distribution of the mean positions $\mathbf{R}_{j}(z)$. We also embed the distribution for the localization lengths, which may depend on the height $z$ :

$$
\mathcal{P}\left(\xi^{2}, z\right)=\frac{1}{Q N} \sum_{j \text { loc. }} \delta\left(\xi^{2}-\xi_{j}^{2}(z)\right) .
$$


So our Ansatz for the order parameter, denoted as $\bar{\Omega}$, becomes:

$$
\begin{aligned}
\bar{\Omega}(\hat{q}, z) & -(1-Q) \delta_{\hat{q}, \hat{0}} \\
& =\frac{1}{N} \sum_{j \text { loc. }} \exp \left(-\frac{\hat{q}^{2} \xi_{j}^{2}(z)}{2}\right) \int \frac{\mathrm{d} \mathbf{R}_{j}(z)}{A} \exp \left(\mathrm{i} \sum_{\alpha=0}^{n} \mathbf{q}^{(\alpha)} \mathbf{R}_{j}(z)\right) \\
& =Q \delta_{\mathbf{q}_{\|}, \mathbf{0}} \int_{0}^{\infty} \mathrm{d} \xi^{2} \mathcal{P}\left(\xi^{2}, z\right) \exp \left(-\frac{\hat{q}^{2} \xi^{2}}{2}\right)
\end{aligned}
$$

and hence:

$$
\bar{\Omega}(\hat{q}, z)=(1-Q) \delta_{\hat{q}, \hat{0}}+Q \delta_{\mathbf{q}_{\|,}, \mathbf{0}} \int_{\xi^{2}, z} \exp \left(-\frac{\hat{q}^{2} \xi^{2}}{2}\right)
$$

where we defined:

$$
\int_{\xi^{2}, z}:=\int_{0}^{\infty} \mathrm{d} \xi^{2} \mathcal{P}\left(\xi^{2}, z\right)
$$

\subsection{The Saddle-Point Equation with Ansatz for $\Omega$}

Now we plug in the Ansatz (3.44) into the saddle point equation (3.35) and get:

$$
(1-Q) \delta_{\hat{q}_{0}, \hat{0}}+Q \delta_{\mathbf{q}_{0 \|}, \mathbf{0}} \int_{\xi^{2}, z_{0}} \exp \left(-\frac{\hat{q}_{0}^{2} \xi^{2}}{2}\right)=\frac{I\left(\hat{q}_{0}, z_{0}\right)}{I(\hat{0}, 0)}
$$

When plugging the Ansatz into $I\left(\hat{q}_{0}, z_{0}\right)$, Eq. (3.36), we note that the primed sum can be turned into a sum over all $\hat{q}$ :

$$
\sum_{\hat{q} \in \mathrm{HRS}}^{\prime} \Delta(\hat{q}) \bar{\Omega}(\hat{q}, z) \mathrm{e}^{-\mathrm{i} \hat{q} \hat{r}(z)}=Q \sum_{\hat{q}} \Delta(\hat{q}) \mathrm{e}^{-\mathrm{i} \hat{q} \hat{r}(z)} \delta_{\mathbf{q}_{\|}, \mathbf{0}} \int_{\xi^{2}, z} \exp \left(-\frac{\hat{q}^{2} \xi^{2}}{2}\right),
$$

because

1. for $\hat{q} \in 1 \mathrm{RS}$, the Ansatz $\bar{\Omega}(\hat{q}, z)=0$ due to the constraint $\delta_{\mathbf{q}_{\|}, \mathbf{0}}$, and

2. the additional $Q$ of the primed sum (see Def. (3.37)) on the left hand side and the term for $\hat{q}=\hat{0}$ on the right hand side cancel out.

Furthermore, looking at the definition of $\Delta(\hat{q})$, Eq. (3.31c), we can simplify the calculation by absorbing the cross-linking length $a$ into the localization length $\xi$ :

$$
\begin{gathered}
\Delta(\hat{q}) \exp \left(-\frac{\hat{q}^{2} \xi^{2}}{2}\right)=\exp \left(-\frac{\hat{q}^{2} a^{2}}{2}\right) \exp \left(-\frac{\hat{q}^{2} \xi^{2}}{2}\right)=\exp \left(-\frac{\hat{q}^{2} \tilde{\xi}^{2}}{2}\right) \\
\text { with } \quad \tilde{\xi}^{2} \equiv \xi^{2}+a^{2}
\end{gathered}
$$


Therefore:

$$
\begin{aligned}
& I\left(\hat{q}_{0}, z_{0}\right)=\int \mathcal{D} \hat{r}(z) \mathrm{e}^{-\mathrm{i} \hat{q}_{0} \hat{r}\left(z_{0}\right)} \\
& \quad \times \exp \left(\phi^{n} \frac{\mu^{2} Q}{L} \int_{0}^{L} \mathrm{~d} z \sum_{\hat{q}} \mathrm{e}^{-\mathrm{i} \hat{q} \hat{r}(z)} \delta_{\mathbf{q}_{\|}, \mathbf{0}} \int_{\xi^{2}, z} \exp \left(-\frac{\hat{q}^{2} \tilde{\xi}^{2}}{2}\right)-H_{\text {align, } 1}^{(n+1)}\{\hat{r}(z)\}\right)
\end{aligned}
$$

\subsubsection{Expansion to second order in $Q$}

The functional integral $I\left(\hat{q}_{0}, z_{0}\right)$, Eq. (3.50), can be calculated to second order in $Q$, as seen in Appendix C.4. Plugging the result for $I\left(\hat{q}_{0}, z_{0}\right)$ back into the saddle point equation (3.46) yields:

$$
\begin{aligned}
Q & \left(1+\mu^{2} Q\right) \int_{\xi^{2}, s_{0}} \exp \left(-\frac{q_{0 \perp}^{2} \xi^{2}}{2}\right) \\
= & \mu^{2} Q \int_{0}^{1} \mathrm{~d} s_{1} \int_{\xi_{1}^{2}, s_{1}} \exp \left(-\frac{q_{0 \perp}^{2}}{2}\left(2 \ell^{2}\left|s_{0}-s_{1}\right|+a^{2}+\xi_{1}^{2}\right)\right) \\
+ & \frac{\left(\mu^{2} Q\right)^{2}}{2} \int_{0}^{1} \mathrm{~d} s_{1} \mathrm{~d} s_{2} \int_{\xi_{1}^{2}, s_{1}} \int_{\xi_{2}^{2}, s_{2}} \\
& \exp \left(\frac{q_{0 \perp}^{2}}{2}\left\{\frac{\left(\ell^{2} g(\mathbf{s})-a^{2}-\xi_{2}^{2}\right)^{2}}{\xi_{1}^{2}+\xi_{2}^{2}+2 a^{2}+2 \ell^{2}\left|s_{1}-s_{2}\right|}-2 \ell^{2}\left|s_{0}-s_{2}\right|-a^{2}-\xi_{2}^{2}\right\}\right) \\
& +\mathcal{O}\left(Q^{3}\right) .
\end{aligned}
$$

Here, we introduced a few simplifying definitions: The dimensionless system height is defined as

$$
s_{\gamma}:=\frac{z_{\gamma}}{L} \in[0,1] \quad \text { for } \quad \gamma=0,1,2 .
$$

Accordingly the integral

$$
\int_{\xi^{2}, s}:=\int_{0}^{\infty} \mathrm{d} \xi^{2} \mathcal{P}\left(\xi^{2}, s\right),
$$

where $\mathcal{P}\left(\xi^{2}, s\right)$ depends on the dimensionless height $s$, but apart from that it is the same as $\mathcal{P}\left(\xi^{2}, z\right)$. Moreover:

$$
g(\mathbf{s}) \equiv g\left(s_{0}, s_{1}, s_{2}\right):=\left|s_{0}-s_{1}\right|-\left|s_{0}-s_{2}\right|-\left|s_{1}-s_{2}\right|
$$

And in addition, the dependence on $L$ and $\sigma$ can be absorbed into

$$
\ell^{2}:=\frac{L}{2 \sigma}
$$

where $\ell$ is the transverse radius of gyration of an uncrosslinked polymer chain (see Sec. 3.2.1).

Eq. (3.51) is correct for arbitrary $q_{\perp 0}$ and $s_{0}$. However the case $q_{\perp 0}=\hat{0}$ was excluded, which trivially yields $1=1$, as can already be seen in the basic saddle point equation (3.35). 


\subsubsection{Sol-Gel Transition}

Eq. (3.51) in the limit $q_{0 \perp}^{2} \rightarrow 0$ yields the well known relation for the gel-fraction Q. (See Sec. 2.9.2, and [Castillo et al., 1994; Goldbart et al., 1996; Broderix et al., 2002].) Here we restrict ourselves to the neighborhood of the sol-gel transition:

$$
Q\left(1+\mu^{2} Q\right)=\mu^{2} Q+\frac{\mu^{4} Q^{2}}{2}+\mathcal{O}\left(Q^{3}\right)
$$

This equation has a trivial solution $Q=0$, the sol phase, known to be stable for $\mu^{2}<1$. For $Q>0$, we can divide by $Q$ to get:

$$
Q=2\left(\mu^{2}-1\right)+\mathcal{O}\left(Q^{2}\right)
$$

which goes to zero when approaching the sol-gel transition, $\mu^{2} \downarrow 1$. Defining $\varepsilon=$ $\mu^{2}-1$, the distance from the transition point, and noting that $Q=\mathcal{O}(\varepsilon)$, as seen in the above equation, we can write:

$$
Q=2 \varepsilon+\mathcal{O}\left(\varepsilon^{2}\right)
$$

which is the same form as for the RLP model (see Eq. (2.69)).

\subsection{The Equation for the Localization Length}

In this section we transform the saddle point equation (3.51) into an integrodifferential equation for the distribution of localization lengths. The basic steps are presented here, while the actual calculation, being a bit intricate and draggy, is deferred to Appendix C.5.

\subsubsection{Normalization of Length Scales}

As we can see in Eq. (3.51), there are three length scales in our system: $\xi, \ell$ and $a$. We can simplify the equations by measuring all lengths in units of $\ell$, i.e. dividing all lengths by $\ell$ and multiplying all $\hat{q}$-vectors with $\ell$. Hence, we define:

$$
\begin{aligned}
\xi_{\ell}^{2} & :=\xi^{2} / \ell^{2} \\
a_{\ell}^{2} & :=a^{2} / \ell^{2} \\
q_{0 \ell \perp}^{2} & :=\ell^{2} q_{0 \perp}^{2}
\end{aligned}
$$

With this scaling there is no explicit dependence on $\ell$ in the saddle point equation anymore. See e.g. Eq. (C.62).

As studies of different randomly cross-linked systems have shown before, the localization length diverges as we approach the sol-gel transition from the gel side [Broderix et al., 2002; Goldbart et al., 1996; Peng et al., 1998; Castillo et al., 1994]. Hence, as a next step, we normalize the localization length with $\varepsilon$, the distance from 
the critical point, which was introduced in Sec. 3.6.2. Thereby, we introduce the new variable, the normalized inverse and squared localization length

$$
\begin{aligned}
& \theta:=\frac{f}{\varepsilon \xi_{\ell}^{2}}=\frac{f \ell^{2}}{\left(\mu^{2}-1\right) \xi^{2}}, \\
& \text { with } \quad f=\frac{2}{3}+\frac{a^{2}}{\ell^{2}}
\end{aligned}
$$

and define the corresponding distribution $\pi(\theta, s)$ for $\theta$ such that:

$$
\mathrm{d} \xi^{2} \mathcal{P}\left(\xi^{2}, s\right)=\mathrm{d} \theta \pi(\theta, s)
$$

As we will see, $\theta$ remains of order $\mathcal{O}(1)$, when we approach the transition point. ${ }^{3}$ Similar approaches have already been used successfully by the four above-mentioned studies to account for the diverging localization length. In our system, however, we have two relevant length scales besides the localization length, which is accounted for by the (dimensionless) factor $f$. The particular choice (3.60b) for $f$ seems arbitrary, but will yield a parameter free equation for the mean distribution of $\theta$.

\subsubsection{Result for the Distribution of Localization Lengths}

With the definitions and concepts of the previous section, we transform the saddle point equation (3.51) into an integro-differential equation for the distribution $\pi(\theta, s)$. The calculation, done in Appendix C.5, yields

$$
\begin{aligned}
& (1+2 \varepsilon) \pi(\theta, s) \\
& =(1+\varepsilon) \int_{0}^{1} \mathrm{~d} s_{1} \pi\left(\theta, s_{1}\right)+\frac{\varepsilon}{f} \int_{0}^{1} \mathrm{~d} s_{1} \partial_{\theta}\left(\theta^{2} \pi\left(\theta, s_{1}\right)\right)\left\{2\left|s-s_{1}\right|+a_{\ell}^{2}\right\} \\
& \quad+\varepsilon \int_{0}^{1} \mathrm{~d} s_{1} \mathrm{~d} s_{2} \int_{0}^{\theta} \mathrm{d} \theta_{1} \pi\left(\theta_{1}, s_{1}\right) \pi\left(\theta-\theta_{1}, s_{2}\right)+\mathcal{O}\left(\varepsilon^{2}\right) .
\end{aligned}
$$

This equation for $\pi(\theta, s)$ is still difficult to handle, since it involves two variables, the normalized inverse localization length $\theta$ and the normalized height $s$. To get a better understanding of the equation, it is useful to define

$$
\bar{\pi}(\theta)=\int_{0}^{1} \mathrm{~d} s \pi(\theta, s)
$$

\footnotetext{
${ }^{3}$ Alternatively we could define $\hat{\xi}^{2} \sim \varepsilon \xi^{2}$, which is also of the order $\mathcal{O}(1)$. However, defining the reciprocal, Eq. (3.60a), turns out to yield a simpler differential equation in the end.
} 
the mean value of the distribution with respect to $s$. Using that definition and by sorting Eq. (3.61) in orders of $\varepsilon$, we get:

$$
\begin{gathered}
\pi(\theta, s)-\bar{\pi}(\theta) \\
=\varepsilon\left(-2 \pi(\theta, s)+\bar{\pi}(\theta)+\frac{1}{f} \int_{0}^{1} \mathrm{~d} s_{1} \partial_{\theta}\left(\theta^{2} \pi\left(\theta, s_{1}\right)\right)\left\{2\left|s-s_{1}\right|+a_{\ell}^{2}\right\}\right. \\
\left.\quad+\int_{0}^{\theta} \mathrm{d} \theta_{1} \bar{\pi}\left(\theta_{1}\right) \bar{\pi}\left(\theta-\theta_{1}\right)\right)+\mathcal{O}\left(\varepsilon^{2}\right) .
\end{gathered}
$$

This equation states that the deviation of the distribution from its mean value is of the order $\varepsilon$. Hence it is suggestive to define $\delta \pi(\theta, s)$ with:

$$
\begin{aligned}
& \pi(\theta, s) \equiv \bar{\pi}(\theta)+\varepsilon \delta \pi(\theta, s), \\
& \text { with } \quad \int_{0}^{1} \mathrm{~d} s \delta \pi(\theta, s)=0,
\end{aligned}
$$

and we are able to calculate both parts, $\bar{\pi}(\theta)$ and $\delta \pi(\theta, s)$, separately to lowest order in $\varepsilon$ :

Therefore we first plug this definition into the right hand side of Eq. (3.63). Since these expressions are already of order $\varepsilon$, we can just replace $\pi(\theta, s)$ by $\bar{\pi}(\theta)$, because the corrections are of the order $\mathcal{O}\left(\varepsilon^{2}\right)$ :

$$
\begin{aligned}
\varepsilon \delta & \pi(\theta, s) \\
= & \varepsilon\left(-\bar{\pi}(\theta)+\frac{1}{f} \int_{0}^{1} \mathrm{~d} s_{1} \partial_{\theta}\left(\theta^{2} \bar{\pi}(\theta)\right)\left\{2\left|s-s_{1}\right|+a_{\ell}^{2}\right\}+\int_{0}^{\theta} \mathrm{d} \theta_{1} \bar{\pi}\left(\theta_{1}\right) \bar{\pi}\left(\theta-\theta_{1}\right)\right) \\
& +\mathcal{O}\left(\varepsilon^{2}\right) .
\end{aligned}
$$

Now it is possible to perform the integration over $s_{1}$ in the second term on the right hand side

$$
\int_{0}^{1} \mathrm{~d} s_{1}\left|s-s_{1}\right|=\frac{s^{2}+(1-s)^{2}}{2} .
$$

Furthermore we plug in $f=\frac{2}{3}+a_{\ell}^{2}$, see Eq. (3.60b), and divide by $\varepsilon$ :

$$
\begin{aligned}
& \delta \pi(\theta, s)= \\
& =-\bar{\pi}(\theta)+\frac{s^{2}+(1-s)^{2}+a_{\ell}^{2}}{2 / 3+a_{\ell}^{2}} \partial_{\theta}\left(\theta^{2} \bar{\pi}(\theta)\right)+\int_{0}^{\theta} \mathrm{d} \theta_{1} \bar{\pi}\left(\theta_{1}\right) \bar{\pi}\left(\theta-\theta_{1}\right)+\mathcal{O}(\varepsilon)
\end{aligned}
$$

This equation can be used to obtain the form of the deviation $\delta \pi(\theta, s)$ form the mean distribution, once we know the mean distribution $\bar{\pi}(\theta)$. In order to get the equation for $\bar{\pi}(\theta)$, we integrate both sides over $s$, use

$$
\int_{0}^{1} \mathrm{~d} s\left(s^{2}+(1-s)^{2}\right)=\frac{2}{3}
$$


for the second term and get:

$$
0=-\bar{\pi}(\theta)+\partial_{\theta}\left(\theta^{2} \bar{\pi}(\theta)\right)+\int_{0}^{\theta} \mathrm{d} \theta_{1} \bar{\pi}\left(\theta_{1}\right) \bar{\pi}\left(\theta-\theta_{1}\right)+\mathcal{O}(\varepsilon),
$$

and hence:

$$
(1-2 \theta) \bar{\pi}(\theta)=\theta^{2} \bar{\pi}^{\prime}(\theta)+\int_{0}^{\theta} \mathrm{d} \theta_{1} \bar{\pi}\left(\theta_{1}\right) \bar{\pi}\left(\theta-\theta_{1}\right)+\mathcal{O}(\varepsilon)
$$

Comparing Eqs. (3.67) and (3.69), we can simplify the equation for the deviation from the mean value:

$$
\delta \pi(\theta, s)=\left(\frac{s^{2}+(1-s)^{2}+a_{\ell}^{2}}{2 / 3+a_{\ell}^{2}}-1\right) \partial_{\theta}\left(\theta^{2} \bar{\pi}(\theta)\right)+\mathcal{O}(\varepsilon),
$$

and hence:

$$
\begin{aligned}
\delta \pi(\theta, s) & =w(s) \partial_{\theta}\left(\theta^{2} \bar{\pi}(\theta)\right)+\mathcal{O}(\varepsilon), \\
\text { with } w(s) & =\frac{s^{2}+(1-s)^{2}-2 / 3}{a^{2} / \ell^{2}+2 / 3}
\end{aligned}
$$

Here we have to keep in mind that the actual localization lengths are related to $\theta$ by

$$
\xi_{\ell}^{2}=\frac{f}{\varepsilon \theta}=\frac{\frac{2}{3}+a_{\ell}^{2}}{\left(\mu^{2}-1\right) \theta}
$$

as defined in Eqs. (3.60), and with Eq. (3.59a):

$$
\xi^{2}=\frac{\frac{2}{3} \ell^{2}+a^{2}}{\left(\mu^{2}-1\right) \theta}
$$

The result for $\bar{\pi}(\theta)$ from Eq. (3.70) can be computed numerically: This is done by discretizing $\bar{\pi}(\theta)$ into points $\bar{\pi}_{j}=\bar{\pi}(j \cdot \Delta \theta)$ with $j=0, \ldots, j_{\max }$ and solving the resulting set of (polynomial) equations. The constraint $\sum_{j=0}^{j_{\max }} \Delta \theta \cdot \bar{\pi}_{j}=1$ has to be taken into account. The result for $\bar{\pi}(\theta)$ obtained by this procedure is plotted in Fig. 3.7.

Indeed Eq. (3.70) for the (normalized and inverse) localization length is well known ${ }^{4}$ : It is also found and investigated for isotropic polymer systems [Broderix et al., 2002; Goldbart et al., 1996].

Here, however, the system is not isotropic, but has the preferred $z$-direction. Since the ends of the chains are loose, the behavior at the top and bottom boundaries

\footnotetext{
${ }^{4}$ Different ways of normalization of the localization lengths may yield different prefactors for the terms of Eq. (3.70), however, they can always be reconciled with Eq. (3.70)
} 


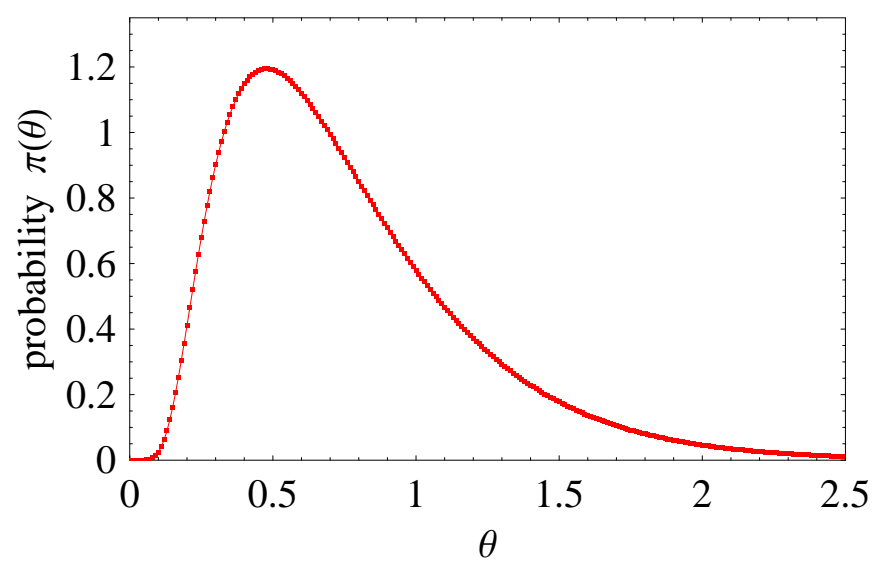

Figure 3.7: Mean probability distribution $\bar{\pi}(\theta)$ of the normalized inverse localization length $\theta$. The curve was obtained by solving the integro-differential equation (3.70) numerically.

is different from the bulk. This difference can be understood in the following way: A chain segment far from the boundaries has a certain probability to have a cross-link above or below. However, if this segment is close to, let's say, the top boundary, the probability for a cross-link to be above is lower. Consequently, since close to the boundary the chains have fewer nearby cross-links, one would expect an increased localization length. This behavior is indeed seen in Fig. 3.8, where the deviation $\delta \pi(\theta, s)$, obtained from Eq. (3.72), is plotted vs. $\theta$ and the system height $s$. One can see that, indeed, close to the boundaries $s \approx 0$ and $s \approx 1$, smaller $\theta$ and hence larger localization lengths $\xi$ are favored. On the other hand, inside the bulk $s \approx 1 / 2$, the opposite is true.

The strength of this inhomogeneity is controlled by the ratio of the two internal length scales $a_{\ell}^{2}=a^{2} / \ell^{2}$. When taking the limit $\ell^{2}=L /(2 \sigma) \rightarrow 0$, i.e. considering thin slices $(L \rightarrow 0)$ or well aligned parallel chains $(\sigma \rightarrow \infty)$, the presented $(D+1)$ dimensional system becomes effectively $D$-dimensional and isotropic. This can also be seen in Eq. (3.72): For $\ell^{2} \rightarrow 0$, the prefactor $w(s) \rightarrow 0$ and hence the deviations of the localization length from its mean value vanish. In this case, the relevant length scale for the localization length is $a$, see Eq. (3.74):

$$
\xi^{2}=\frac{a^{2}}{\left(\mu^{2}-1\right) \theta} \quad \Rightarrow \quad \xi \propto \frac{a}{\sqrt{\mu^{2}-1}} .
$$

The other and more relevant case is the limit of large system heights $L \rightarrow \infty$. In this case the relevant length scale for the localization length is the radius of gyration $\ell^{2}=L /(2 \sigma)$ of the chains (perpendicular to the $z$-axis); the cross-linking length $a$ becomes unimportant:

$$
\xi^{2}=\frac{2}{3} \frac{\ell^{2}}{\left(\mu^{2}-1\right) \theta} \quad \Rightarrow \quad \xi \propto \frac{\sqrt{L}}{\sqrt{\mu^{2}-1}} .
$$



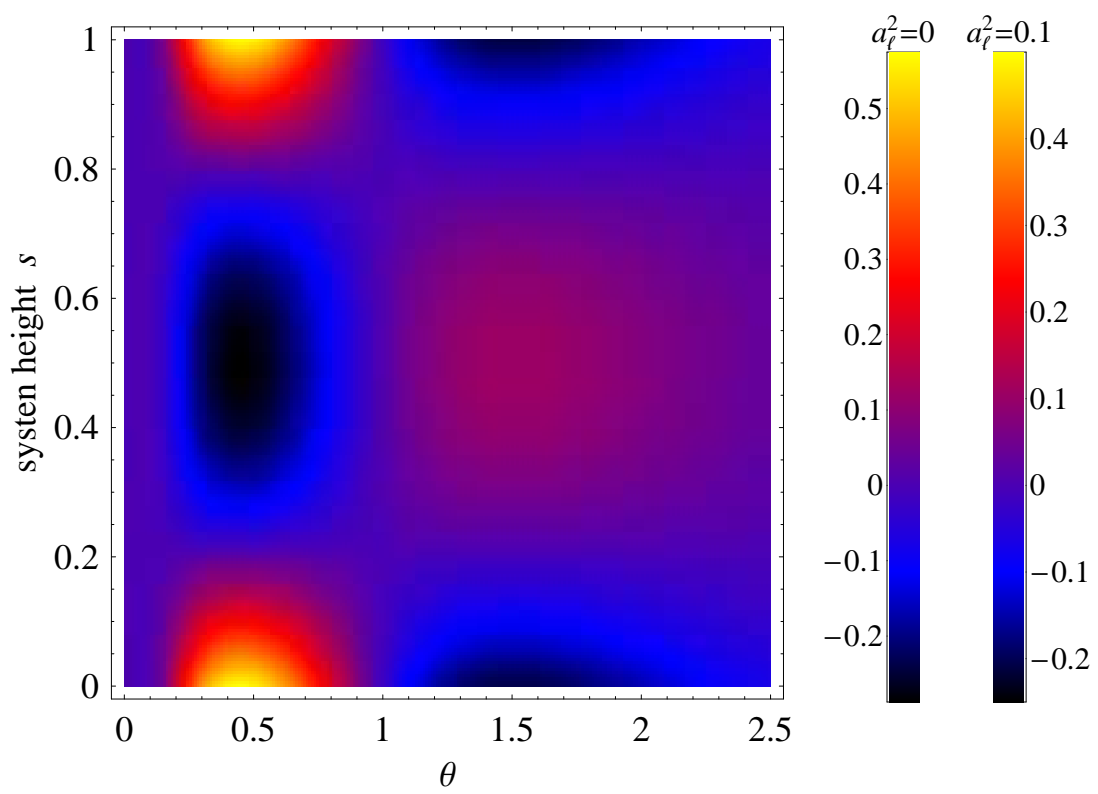

Figure 3.8: Deviation $\delta \pi(\theta, s)$ of the probability distribution of $\theta$ from its mean value, as defined in Eq. (3.64a). The plot was obtained by applying the data from Fig. 3.7 to Eq. (3.72). For different ratios $a_{\ell}^{2}=a^{2} / \ell^{2}, \delta \pi(\theta, s)$ can be rescaled simply resulting in different color bars.

One can also see that larger system heights $L$ yield larger localization lengths, since, due to a larger radius of gyration $\ell \propto \sqrt{L}$, the chains can span a larger region in the sample. Also the deviation $\delta \pi(\theta, s)$ is most dominant in this large- $L$ regime (see Eq. (3.72) and compare color bars of Fig. 3.8).

As a last thing, it is instructive to see how the typical localization length is affected by the deviation $\delta \pi(\theta, s)$. For that we will need the first two moments of the mean distribution $\bar{\pi}(\theta)$ :

$$
\bar{\theta}=\int_{0}^{\infty} \mathrm{d} \theta \theta \bar{\pi}(\theta) \quad \text { and } \quad \overline{\theta^{2}}=\int_{0}^{\infty} \mathrm{d} \theta \theta^{2} \bar{\pi}(\theta) .
$$

With that we can easily calculate the average inverse localization length:

$$
\begin{aligned}
& \theta_{\mathrm{typ}}(s):=\int_{0}^{\infty} \mathrm{d} \theta \theta \pi(\theta, s)=\int_{0}^{\infty} \mathrm{d} \theta \theta \bar{\pi}(\theta)+\varepsilon \int_{0}^{\infty} \mathrm{d} \theta \theta \delta \pi(\theta, s)= \\
& \stackrel{\text { Eq. }}{=}{ }^{(3)} \bar{\theta}+\varepsilon w(s) \int_{0}^{\infty} \mathrm{d} \theta \theta \partial_{\theta}\left(\theta^{2} \bar{\pi}(\theta)\right)+\mathcal{O}\left(\varepsilon^{2}\right)= \\
&=\bar{\theta}+\varepsilon w(s)\left(\left.\theta \cdot \theta^{2} \bar{\pi}(\theta)\right|_{0} ^{\infty}-\int_{0}^{\infty} \mathrm{d} \theta \theta^{2} \bar{\pi}(\theta)\right)+\mathcal{O}\left(\varepsilon^{2}\right) .
\end{aligned}
$$

As stated by Castillo et al. [1994], the mean distribution $\bar{\pi}(\theta)$, coming from Eq. (3.70), should fall off exponentially for $\theta \rightarrow \infty$. Hence we can omit the boundary term $\left.\theta^{3} \bar{\pi}(\theta)\right|_{0} ^{\infty}$ and get:

$$
\theta_{\text {typ }}(s)=\bar{\theta}-\varepsilon \overline{\theta^{2}} w(s)+\mathcal{O}\left(\varepsilon^{2}\right) .
$$


And hence, the typical localization length becomes:

$$
\xi_{\text {typ }}^{2}(s):=\frac{f \ell^{2}}{\varepsilon \theta_{\text {typ }}(s)}=\frac{\frac{2}{3} \ell^{2}+a^{2}}{\varepsilon \bar{\theta}}\left(1+\varepsilon w(s) \frac{\overline{\theta^{2}}}{\bar{\theta}}\right)+\mathcal{O}(\varepsilon)
$$

On the right hand side of this equation, the prefactor $\left(\frac{2}{3} \ell^{2}+a^{2}\right) / \varepsilon$ sets the length scale for the localization length, which diverges in the limit $\varepsilon \rightarrow 0$. $(\bar{\theta}$ is just a dimensionless number of order 1.) In the parentheses, the dominant term " 1 " does not incorporate spatial dependencies, yielding similar behavior as for isotropic systems. Relative to this term, the $s$-dependent second term provides corrections of order $\varepsilon$. Hence these anisotropic corrections are of the same order as $\frac{2}{3} \ell^{2}+a^{2}$ and do not diverge or vanish in the limit $\varepsilon \rightarrow 0$.

Furthermore we have to note that the definition of the typical localization length $\xi_{\text {typ }}^{2}(s)$ is, for simplification, just the inverse of $\theta_{\text {typ }}(s)$, and thus it is not exactly the the mean of the distribution $\mathcal{P}\left(\xi^{2}, s\right)$.

\subsection{Conclusions}

In this chapter we investigated a system of cross-linked directed polymer chains in a $(D+1)$-dimensional volume $L \cdot A$, where $L$ is the height in $z$-direction, along which the chains are preferably aligned, and $A$ is the base area.

The chains are subject to an alignment interaction, which penalizes tilting of the chains with respect to the $z$-axis. The strength $\sigma$ of this interaction may be interpreted as the force $f$ with which a worm-like chain is stretched along the $z$ axis, or as the coupling strength of the polymers to a spatially uniform nematic matrix. This interaction constitutes the typical extension of an uncrosslinked chain perpendicular to the $z$-axis, $\ell=\sqrt{L /(2 \sigma)}$.

The cross-linking interaction permanently connects random pairs of chains at a fixed height, with a cross-link density determined by the parameter $\mu^{2}$. This interaction introduces a second length scale, the typical cross-link length $a$.

The system exhibits a continuous phase transition - the sol-gel transition - as the cross-link concentration is increased beyond the critical value $\mu_{\mathrm{c}}^{2}=1$. This transition is characterized by the gel-fraction $Q$, which vanishes like $Q \propto\left(\mu^{2}-\mu_{\mathrm{c}}^{2}\right)$ as the transition point is approached from the gel phase $\mu^{2} \downarrow \mu_{\mathrm{c}}^{2}$. Thereby the typical localization length diverges as $\xi_{\text {typ }} \propto\left(\mu^{2}-\mu_{\mathrm{c}}^{2}\right)^{-1 / 2}$. The relevant length scale is the transverse extension $\ell=\sqrt{L /(2 \sigma)}$ of the chains, given that $\ell \gg a$.

The distribution of localization lengths varies throughout the height $z$ of the system: Close to the boundaries $z \approx 0$ and $z \approx L$, larger localization lengths are favored. This is due to the fact the ends of the chains are loose and hence, the probability that a given chain segment has nearby cross-links is lower if this segment is close to a boundary.

As an outlook, it would be interesting to calculate mechanical properties of the system like it was done for the randomly cross-linked particle model. Since 
the system is not isotropic, the shear modulus, describing the energy cost of a density conserving deformation of the type $\nabla_{\mathbf{r}} \mathbf{u}(\mathbf{r}, z)$, is expected to differ from the tilt modulus, accounting for deformations like $\partial_{z} \mathbf{u}(\mathbf{r}, z)$. The latter is expected to remain non-zero in the liquid phase (even in the totally uncrosslinked phase), because of the energy cost of tilting due to the alignment interaction. Also the bulk modulus, describing the energy cost of density fluctuations, would be interesting to calculate; this could be done by incorporating a pressure field to the Hamiltonian $H_{\text {align }}$ (as proposed in Eq. (C.14)) and investigate the response of the system to pressure fluctuations.

Another matter would be to evaluate the saddle-point equation (3.46) to higher or even infinite order, as it was possible for the RLP model in Chapter 2. It would be interesting to see how stronger cross-linking affects the inhomogeneity of the localization lengths close to the boundaries, and if higher orders of the mean distribution $\bar{\pi}(\theta)$ are in accordance with corresponding isotropic models.

An extension to the model would be to incorporate cross-link at different heights $z_{e}$ and $z_{e}^{\prime}$. This would be relevant, since recent developments suggest that the tilt modulus is not influenced by cross-links which are fixed at the same height on both chains, as in the presented model. [Ulrich et al., 2010]

Another modification is the introduction of so called "slip-links": Thereby the quenched cross-link configuration only specifies which pairs of chains are connected. The height of the cross-link $z_{e}$, however, is not quenched but may move along the chains, as they fluctuate around. These slip-links can be seen as a means to account for fixed entanglements of the chains. In this respect, an entanglement can move along a pair of chains, but these chains cannot be disentangled. 
CHAPter 4

\title{
Structure of Spider Silk Modeled as a Random Network
}

\author{
Contents \\ 4.1 Introduction $\ldots \ldots \ldots \ldots \ldots \ldots \ldots \ldots \ldots \ldots$ \\ 4.2 Model ........................ 60 \\ 4.2 .1 Unit Cell . . . . . . . . . . . . . . . . . . . . . 60

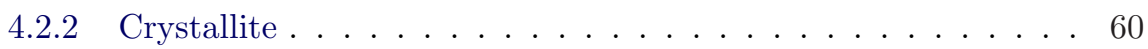 \\ 4.2 .3 Ensemble of Crystallites . . . . . . . . . . . . . . . . 63 \\ 4.2 .4 Continuous Background . . . . . . . . . . . . . 64
}

4.3 Scattering Function . . . . . . . . . . . . 65

4.3.1 Incoherent Part $G_{1}(\mathbf{q}) \ldots \ldots \ldots \ldots 6$

4.3 .2 Coherent Part $G_{2}(\mathbf{q}) \ldots \ldots \ldots \ldots \ldots$

4.3.3 Scattering Amplitude of a Single Crystallite . . . . . . . . . . 68

4.4 Atomic Configuration of the Unit Cell . . . . . . . . . . 69

4.4 .1 Unshifted Unit Cells . . . . . . . . . . . . . . . . . . 69

4.4.2 Possible Shifts inside the Unit Cell . . . . . . . . . . . . . . 72

4.4.3 Variations between Crystallites . . . . . . . . . . . . . 72

4.5 Results . . . . . . . . . . . . . . . 73

4.5.1 Experimental Scattering Function _ . . . . . . . . . 73

4.5.2 Scattering Function from the Model . . . . . . . . . . . 74

4.6 Conclusions . . . . . . . . . . . . . . 79

\subsection{Introduction}

Spider silk has long been widely known, but has only recently received great appreciation from the scientific community for its outstanding material properties [Grubb \& Jelinski, 1997; Fossey \& Kaplan, 1999]. In general, a spider is capable of producing a variety of different types of silk fibers; scientific interest here has focused on the so-called dragline fibers, fibers known for their high strength. Orb web spiders produce them essentially from only two proteins and use them to build their net's frame and radii, and also to support their own body weight after an intentional fall during escape from attack. Evolution has optimized dragline fibers for tensile 
strength (i.e. the stress at which the material breaks), extensibility and tenacy (i.e. the energy per volume dissipated before the material breaks, a.k.a. toughness). Dragline silk can support large strains and has a tensile strength comparable to steel or Kevlar. Regarding tenacy, values of $160 \mathrm{MJ} / \mathrm{m}^{3}$ have been reported [Kaplan et al., 1994; Gosline et al., 1999], e.g. for different Nephila species, on which most studies have been carried out.

An understanding of the structural origins of these mechanical properties is of fundamental interest, and may at the same time serve the development of biomimetic material design [Huemmerich et al., 2004; Scheibel, 2004] using recombinant and synthetic approaches [Huemmerich et al., 2006; Foo et al., 2006; Rammensee et al., 2006]. As for other biomaterials, the correlation between structure and the mechanical properties can only be clarified by advanced structural characterization accompanied by numerical modeling. To this end, not only the mechanical properties [Vollrath \& Porter, 2006; Zbilut et al., 2006] resulting from the structure, but also the structure itself has to be modeled to interpret the experimental data. Such efforts have in the past led to a quantitative understanding of many biomaterials like bone, tendons and wood [Roschger et al., 2001; Puxkandl et al., 2002].

As deduced from X-ray scattering [Warwicker, 1960; Kaplan et al., 1994; Grubb \& Jelinski, 1997] and NMR experiments [van Beek et al., 2002], spider silks are characterized by a seemingly simple design (see Fig. 4.1): The alanine-rich segments of the fibroin polypeptide chains fold into $\beta$-sheet nano-crystallites (similar to polyL-alanine crystals), which are embedded in an amorphous network of chains, containing predominately glycine. In this polymer network, the crystalline component makes up an estimated $20 \%-30 \%$ of the total volume, and may be considered as cross-links, interconnecting several different chains.

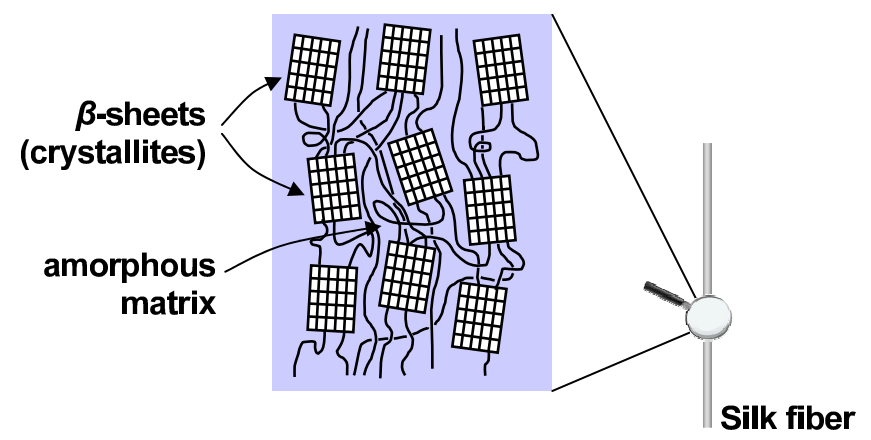

Figure 4.1: Schematic illustration of dragline silk. $\beta$-sheets, mainly composed of polyalanine, are embedded in the amorphous matrix, an amorphous network of chains.

The detailed investigation of the structure is complicated, at least on the single fiber level, by the relatively small diameters in the range of $1-10 \mu \mathrm{m}$, depending on the species. Using highly brilliant microfocused synchrotron radiation, diffraction patterns can be obtained not only on thick samples of fiber bundles, but also on a single fiber [Riekel et al., 1999a,b, 2000; Riekel \& Vollrath, 2001; Riekel et al., 2004; 
Sapede et al., 2005]. Single fiber diffraction has been used under simultaneous controlled mechanical load, in order to investigate changes of the molecular structure with increasing strain up to failure [Glišović et al., 2008]. Single fiber diffraction, where possible, is much better suited to correlate the structure to controlled mechanical load, since the strain distribution in bundles is intrinsically inhomogeneous, and the majority of load may be taken up by a small minority of fibers.

While progress of the experimental diffraction studies has been evident, the analysis of the data still relies on the classical classification and indexing scheme introduced by Warwicker. According to Warwicker, the $\beta$-sheet crystallites of the dragline of Nephila fall into the so-called system 3 of a nearly orthorhombic unit cell [Marsh et al., 1955a; Warwicker, 1960] with lattice constants $10.6 \AA \times 9.44 \AA \times 6.95 \AA$ [Warwicker, 1960]. To fix the coordinate system, they define the $x$-axis to be in the direction of the amino acid side chains connecting different $\beta$-sheets, while the $y$ axis denotes the direction along the hydrogen bonds of the $\beta$-sheets. Finally, the $z$-axis corresponds to the axis along the covalent peptide bonds (main chain). The main chain is preferably aligned along the fiber axis (for an illustration, see top panel of Fig. 4.8 on page 71). Note that while we follow this common convention, other notations and choices of axes are also used in literature.

While helpful, the indexing scheme does not give information regarding the exact structure of the unit cell, whether the $\beta$-pleated sheets are composed of parallel or antiparallel strands, or how the two-dimensional sheets are arranged to stacks. To this end, not only peak positions but the entire rather broad intensity distribution has to be analyzed. To interpret the scattering image, it is essential to know whether correlations between different crystallites are important, or whether the measured data can be accounted for by the scattering of single crystallites averaged over fluctuating orientations. It is also not clear, if correlations between translational and rotational degrees of freedom are important. Finally, the powder averaging taking into account the fiber symmetry experimental mosaicity (orientational distribution) must be quantitatively taken into account.

In this chapter a scattering model based on kinematic scattering theory is developed and the numerically calculated scattering intensity is compared with the experimental wide angle scattering distribution measured from aligned silk fibers. The numerical calculations allow for a quantitative comparison to the experimental data and yield both structural and statistical parameters.

Note that the small size of crystallites, leading to correspondingly broad reflections and a generally rather low number of external peaks, exclude a standard crystallographic approach. The presented model is based on a quite general approach, independently allowing for correlations between center-of-mass positions (translations) and varying crystallite orientations (rotations). The structural parameters concern the crystal structure, in particular the atomic positions in the unit cell, and the crystallite size. The statistical parameters relate to the orientational distribution of the crystallite symmetry axis with respect to the fiber axis and the correlations between crystallites.

This chapter is organized as follows: In Sec. 4.2 the basic model is introduced 
with parameters for the crystallite size, lattice constants and statistical parameters for the crystallites' position and orientation. Subsequently, in Sec. 4.3 we compute the scattering function for this model. Sec. 4.4 specifies the different atomic configurations which are conceivable for polyalanine. The main results and the comparison of calculated and measured intensities are presented in Sec. 4.5, before conclusion in Sec. 4.6. Most of the presented results can also be found in [Ulrich et al., 2008].

\subsection{Model}

In this section we develop a simple model of spider silk which allows us to compute the scattering function

$$
G(\mathbf{q})=\left\langle\left|\sum_{j} f_{j} \exp \left(\mathrm{iqr}_{j}\right)\right|^{2}\right\rangle
$$

as measured in X-ray scattering experiments. The atomic positions are denoted by $\mathbf{r}_{j}$ and the atomic form factors by $f_{j}$. The modeling proceeds on three different levels:

1) On the largest length scales, spider silk is modeled as an ensemble of crystallites embedded in an amorphous matrix and preferentially oriented along the fiber axis.

2) Each crystallite is composed of parallel or antiparallel $\beta$-sheets.

3) Each unit cell contains a given number of amino acids, whose arrangements have been classified by Warwicker [1960]. In Fig. 4.2 (right) we see an illustration of Bombyx mori by Geis [Zubay, 1998].

In the following we shall build up a model, starting with the smallest scales and working up to the whole system. Subsequently, we will compare the calculated scattering functions with experimental data. Thereby, we are able to determine the arrangement of atoms in the unit cell which optimizes the agreement between model and experiment.

\subsubsection{Unit Cell}

One unit cell of a crystallite is described as a set of atoms at positions $\mathbf{r}_{k}$ relative to the center of the unit cell, where $k=1,2, \ldots, K$ runs through the atoms of the unit cell (see Fig. 4.2, left panel, for a schematic drawing). Each atom is assigned a form factor $f_{k}$ specifying the scattering strength of the respective atom type.

\subsubsection{Crystallite}

A crystallite is composed of $M=M_{x} M_{y} M_{z}$ unit cells, replicated $M_{x}, M_{y}$ and $M_{z}$ times along the primitive vectors $\mathbf{a}_{x}, \mathbf{a}_{y}$ and $\mathbf{a}_{z}$ respectively. The unit cells in a crystallite are numbered by a vector index $\mathbf{m}=\left(m_{x}, m_{y}, m_{z}\right)$, where $m_{\nu}=$ 

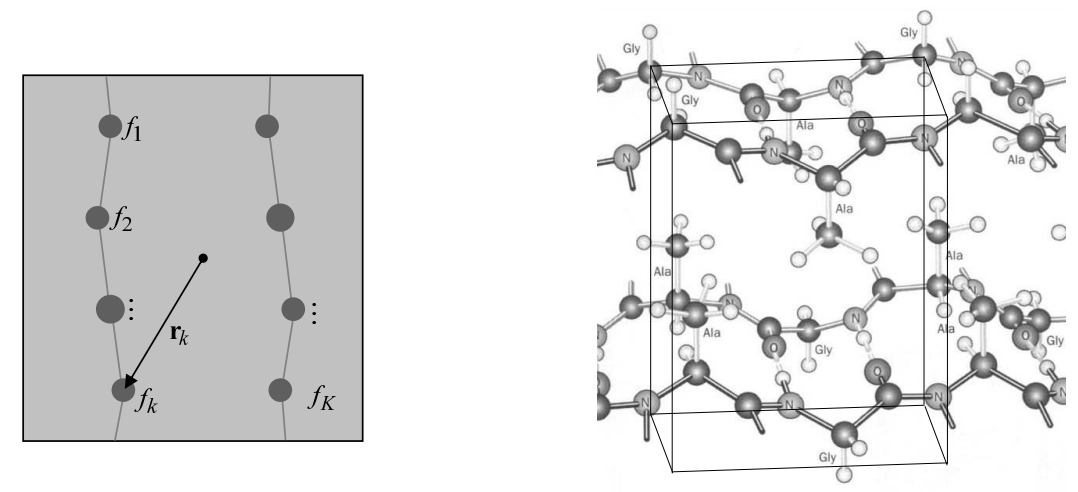

Figure 4.2: Left: Schematic view of the unit cell. Atom $k$ is located at position $\mathbf{r}_{k}$ and the atom type is specified by the form factor $f_{k}$ of the atom. For simplification schematic illustrations are in $2 \mathrm{D}$, if possible, even though the model refers to three space dimensions, $D=3$. Right: A possible configuration inside the unit cell (illustration adapted from [Zubay, 1998]).

$1,2, \ldots, M_{\nu}$ for $\nu=x, y, z$. Hence the center of mass of unit cell $\mathbf{m}$ has position vector

$$
\tilde{\mathbf{s}}_{\mathbf{m}}=m_{x} \mathbf{a}_{x}+m_{y} \mathbf{a}_{y}+m_{z} \mathbf{a}_{z}
$$

In fact, it is more convenient to measure all distances with respect to the center of the whole crystallite

$$
\mathbf{s}_{\mathrm{cm}}=\frac{\left(M_{x}+1\right) \mathbf{a}_{x}+\left(M_{y}+1\right) \mathbf{a}_{y}+\left(M_{z}+1\right) \mathbf{a}_{z}}{2}
$$

so that $\mathbf{s}_{\mathbf{m}}=\tilde{\mathbf{s}}_{\mathbf{m}}-\mathbf{s}_{\mathrm{cm}}$ denotes the position of unit cell $\mathbf{m}$ relative to the center of the respective crystallite (see Fig. 4.3). The position of atom $k$ in unit cell $\mathbf{m}$ relative to the center of the crystallite is

$$
\mathbf{r}_{\mathbf{m}, k}=\mathbf{s}_{\mathbf{m}}+\mathbf{r}_{k}
$$




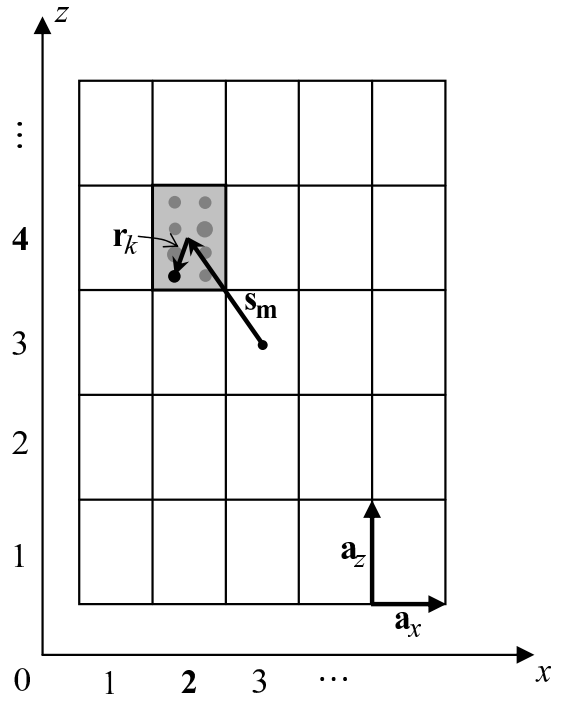

Figure 4.3: Schematic view of the crystallite. The vector $\mathbf{s}_{\mathbf{m}}$ points from the center of the crystallite to the unit cell $\mathbf{m}$. From there, $\mathbf{r}_{k}$ points to atom $k$. 


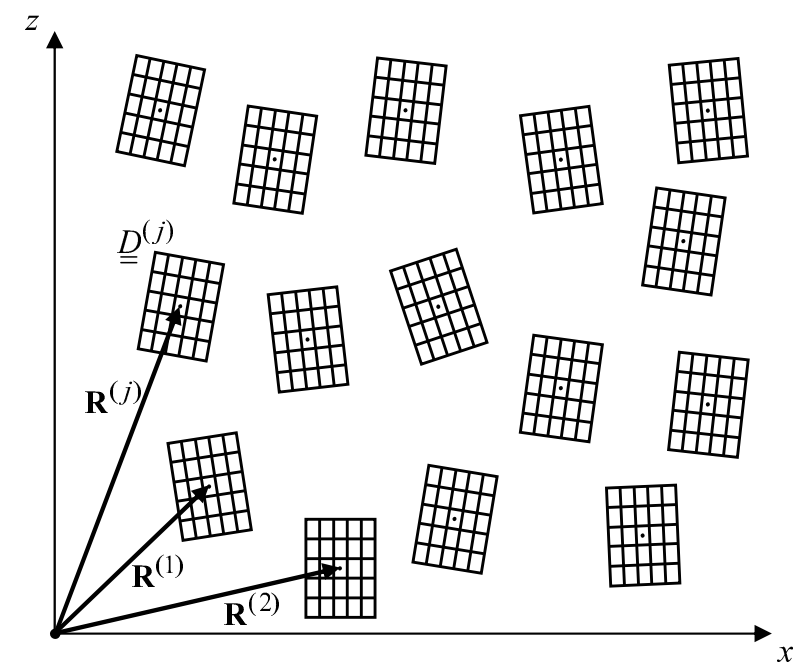

Figure 4.4: The whole system is composed of crystallites at positions $\mathbf{R}^{(j)}$. They can be rotated by rotation matrices $\underline{\underline{D}}^{(j)}$.

\subsubsection{Ensemble of Crystallites}

The whole system is composed of $N$ such crystallites at positions $\mathbf{R}^{(j)}$ with $j=$ $1,2, \ldots, N$ (see Fig. 4.4). The crystallites are not perfectly aligned with the fiber axis, but their orientations fluctuate. The orientation of a single crystallite is specified by three Euler angles $\phi^{(j)}, \theta^{(j)}, \psi^{(j)}$ (see right panel of Fig. 4.5). Here the $z$-axis is the fiber axis and $\theta$ denotes the angle between the $z$-direction of the crystallites (direction of the covalent bonds) and the fiber axis. The atomic positions of the rotated crystallite are obtained from the configuration which is perfectly aligned with the $z$-axis, by applying a rotation matrix $\underline{\underline{D}}^{(j)}$ (see left panel of Fig. 4.5):

$$
\mathbf{r}_{\mathbf{m}, k}^{(j)}=\mathbf{R}^{(j)}+\underline{\underline{D}}^{(j)} \mathbf{r}_{\mathbf{m}, k}
$$

In the experiment, the scattering intensity is obtained for a large system consisting of many crystallites. Hence, it is reasonable to assume that the scattering function is self-averaging and therefore can be averaged over the positions and orientations of the crystallites. Angular brackets \langle\rangle denote the average of an observable $\mathcal{A}$ over crystallite positions $\mathbf{R}^{(j)}$ and orientations $\underline{\underline{D}}^{(j)}$ :

$$
\langle\mathcal{A}\rangle=\int \prod_{j=1}^{N}\left(\mathrm{~d} \mathbf{R}^{(j)} \mathcal{D} \underline{\underline{D}}^{(j)}\right) \mathcal{P}_{\mathrm{pos}}\left(\mathbf{R}^{(1)}, \ldots, \mathbf{R}^{(N)}\right) \mathcal{A}
$$

Here, the crystallite positions follow the distribution function $\mathcal{P}_{\text {pos }}\left(\mathbf{R}^{(1)}, \ldots, \mathbf{R}^{(N)}\right)$, which in general includes correlations. In contrast, the 

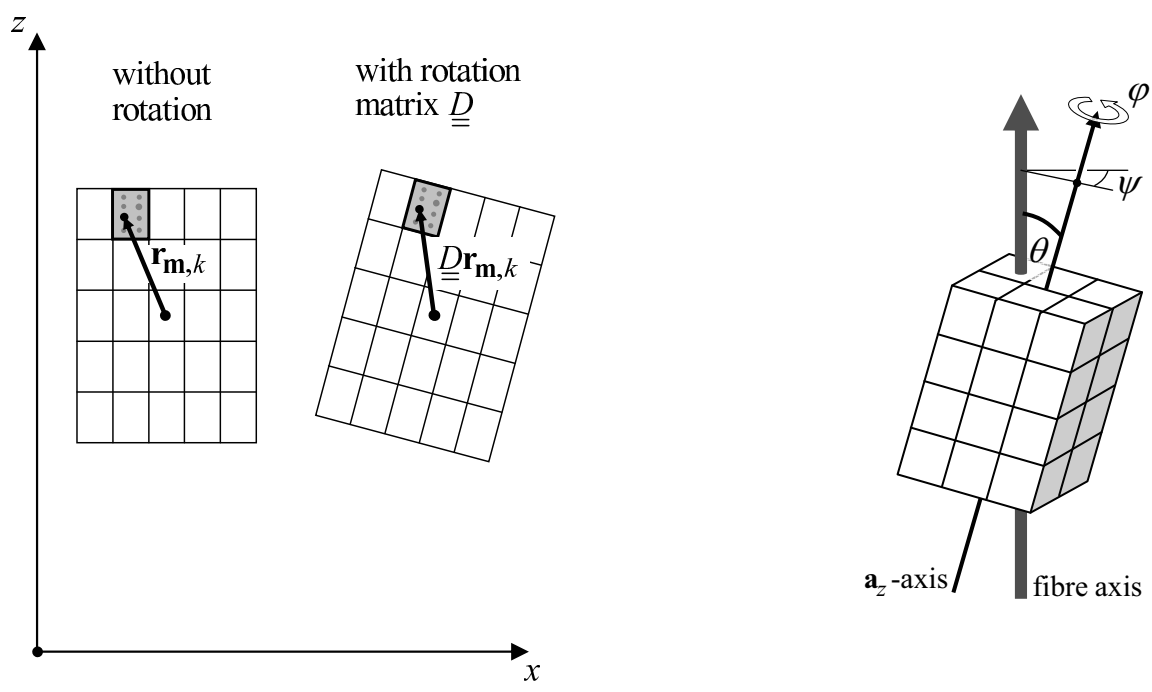

Figure 4.5: Left: The position of atom $k$ in unit cell $\mathbf{m}$ of the rotated crystallite is obtained by applying the rotation matrix $\underline{\underline{D}} \mathbf{r}_{\mathbf{m}, k}$ to the position vector in the aligned configuration. Right: Illustration of Euler angles in 3D. The angle $\theta$ specifies the deviation of the crystallite's $\mathbf{a}_{z}$-axis from the fiber axis of the strand. $\phi$ is the rotation of the crystallite about its own $\mathbf{a}_{z}$-axis, and $\psi$ is the rotation of the $\mathbf{a}_{z}$-axis about the fiber axis (after the $\theta$-rotation).

orientation of each crystallite is assumed to be independent of the others. The average over all orientations

$$
\mathcal{D} \underline{\underline{D}}^{(j)}=\mathrm{d} \phi^{(j)} \mathrm{d} \theta^{(j)} \mathrm{d} \psi^{(j)} \sin \theta^{(j)} \mathcal{P}_{\text {angle }}\left(\phi^{(j)}, \theta^{(j)}, \psi^{(j)}\right)
$$

involves the angular distribution function $\mathcal{P}_{\text {angle }}(\phi, \theta, \psi)$, which is the same for each crystallite. In the simplest model we assume a Gaussian distribution for the deviations of the crystallite axis from the fiber axis $\mathcal{P}_{\text {angle }}(\phi, \theta, \psi) \propto \exp \left(-\theta^{2} / \theta_{0}^{2}\right)$, while all values of $\phi$ and $\psi$ between 0 and $2 \pi$ are equally likely.

\subsubsection{Continuous Background}

The space between the crystallites is filled with water molecules and strands connecting the crystallites, i.e. the amorphous matrix. In the scope of this work, we are not interested in the details of its structure and thus we model it as a continuous background density $\varrho_{0}$, chosen to match the average scattering density of the crystallite (see Fig. 4.6): $\varrho_{0}=\sum_{k=1}^{K} f_{k} / V_{\mathrm{uc}}$. Here $V_{\mathrm{uc}}$ is the volume of the unit cell. There is no background intensity inside the crystallite, achieved in our model by cutting out a spherical cavity $V(\mathbf{r})$ around each atom $\mathbf{r}=\mathbf{r}_{\mathbf{m}, k}^{(j)}$. For simplicity we assume a Gaussian cavity

$$
V(\mathbf{r})=\frac{\bar{f}}{\left(2 \pi \xi^{2}\right)^{3 / 2}} \exp \left(-\frac{\mathbf{r}^{2}}{2 \xi^{2}}\right)
$$




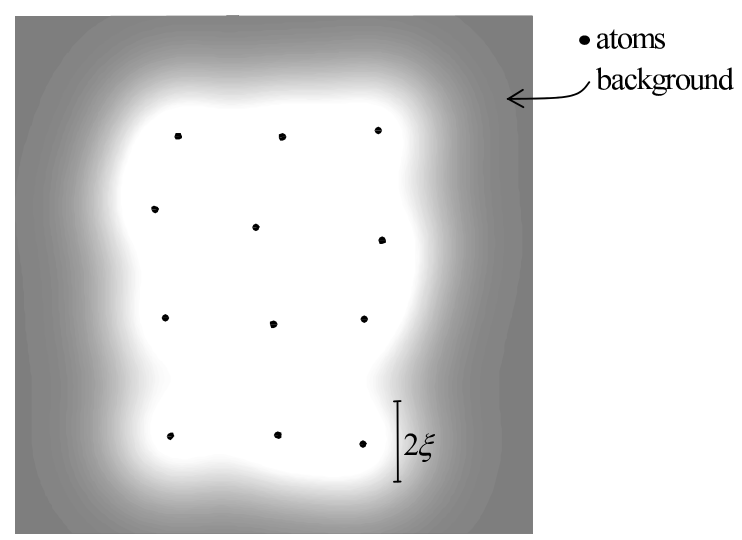

Figure 4.6: Illustration of the continuous background, modeling the amorphous matrix. Outside the crystallites it has a homogeneous scattering density $\varrho_{0}$, matching the mean scattering density of the crystallites. In this illustration $\xi$ is 0.45 of the mean nearest-neighbor distance.

and choose the amplitude such that the average density inside the crystallites is zero:

$$
0=\int_{\text {crystallite }} \mathrm{d} \mathbf{r}\left(\varrho_{0}-\sum_{\mathbf{m}=\mathbf{1}}^{\mathbf{M}} \sum_{k=1}^{K} V\left(\mathbf{r}-\mathbf{r}_{\mathbf{m}, k}\right)\right)
$$

where the sum over the vector index $\sum_{\mathbf{m}=\mathbf{1}}^{\mathbf{M}}$ means $\sum_{m_{x}=1}^{M_{x}} \sum_{m_{y}=1}^{M_{y}} \sum_{m_{z}=1}^{M_{z}}$.

Using the assumption (4.9), $\bar{f}$ becomes simply the average form factor,

$$
\bar{f}=\frac{1}{K} \sum_{k=1}^{K} f_{k}
$$

as can be easily verified. The typical size of the cavity $\xi$ has to be comparable to the nearest-neighbor distance to make sure that there is no "background" inside the crystallites. Models both with and without continuous background are compared in Appendix E.1.

This completes the specification of our model. We now proceed to compute the scattering function as predicted by the model.

\subsection{Scattering Function}

Given the atomic positions $\mathbf{r}_{\mathbf{m}, k}^{(j)}$, the background density $\varrho(\mathbf{r})$ and the statistics of the crystallites' orientations and positions, we calculate the scattering function:

$$
G(\mathbf{q})=\left\langle\left|\sum_{j=1}^{N} \sum_{\mathbf{m}=\mathbf{1}}^{\mathbf{M}} \sum_{k=1}^{K} f_{k} \exp \left(\mathrm{iqr}_{\mathbf{m}, k}^{(j)}\right)+\int \mathrm{d} \mathbf{r} \varrho(\mathbf{r}) \exp (\mathbf{i q r} \mathbf{r})\right|^{2}\right\rangle .
$$


Here, $\varrho(\mathbf{r})$ is the background intensity whose Fourier transform reads as:

$$
\begin{aligned}
\int \mathrm{d} \mathbf{r} \varrho(\mathbf{r}) \exp (\mathbf{i q r}) & =\int \mathrm{d} \mathbf{r} \exp (\mathrm{iqr})\left(\varrho_{0}-\bar{f} \sum_{j=1}^{N} \sum_{\mathbf{m}=\mathbf{1}}^{\mathbf{M}} \sum_{k=1}^{K} V\left(\mathbf{r}-\mathbf{r}_{\mathbf{m}, k}^{(j)}\right)\right) \\
& =\varrho_{0} V \delta_{\mathbf{q}, \mathbf{0}}-\tilde{V}(\mathbf{q}) \sum_{j=1}^{N} \sum_{\mathbf{m}=\mathbf{1}}^{\mathbf{M}} \sum_{k=1}^{K} \exp \left(\mathbf{i q r} \mathbf{r}_{\mathbf{m}, k}^{(j)}\right),
\end{aligned}
$$

with $\tilde{V}(\mathbf{q})$ being the Fourier transform of the cavity $V(\mathbf{r})$. The uniform density $\varrho_{0}$, giving rise to a contribution proportional to $\delta_{\mathbf{q}, \mathbf{0}}$, does not contain information about the structure of the system. Furthermore, the central beam has to be gated out in the analysis of the experimental data. Hence, we neglect the uniform contribution and obtain for the scattering intensity (4.11)

$$
G(\mathbf{q})=\left\langle|\sum_{j=1}^{N} \sum_{\mathbf{m}=\mathbf{1}}^{\mathbf{M}} \sum_{k=1}^{K} \underbrace{\left(f_{k}-\tilde{V}(\mathbf{q})\right)}_{F_{k}(\mathbf{q})} \exp \left(\mathrm{iq} \cdot \mathbf{r}_{\mathbf{m}, k}^{(j)}\right)|^{2}\right\rangle .
$$

The cavities give rise to effective form factors $F_{k}(\mathbf{q})=f_{k}-\tilde{V}(\mathbf{q})$ accounting for the scattering of the atoms themselves, $f_{k}$, and the cavities surrounding them, $\tilde{V}(\mathbf{q})$. Note that, if we want to switch off the background $\bar{f} \rightarrow 0$, we return to the original form factors $F_{k}(\mathbf{q}) \rightarrow f_{k}$.

Inserting the average \langle\rangle and multiplying out the magnitude squared in (4.13) yields:

$$
\begin{aligned}
G(\mathbf{q})=\int & \prod_{j=1}^{N}\left(\mathrm{~d} \mathbf{R}^{(j)} \mathcal{D} \underline{\underline{D}}^{(j)}\right) \mathcal{P}_{\mathrm{pos}}\left(\mathbf{R}^{(1)}, \ldots, \mathbf{R}^{(N)}\right) \\
& \times \sum_{j, j^{\prime}=1}^{N} \exp \left(\operatorname{iq}\left(\mathbf{R}^{(j)}-\mathbf{R}^{\left(j^{\prime}\right)}\right)\right) \\
& \times \sum_{\mathbf{m}, \mathbf{m}^{\prime}=1}^{\mathbf{M}} \sum_{k, k^{\prime}=1}^{K} F_{k}(\mathbf{q}) F_{k^{\prime}}^{*}(\mathbf{q}) \exp \left(\operatorname{iq}\left(\underline{\underline{D}}^{(j)} \mathbf{r}_{\mathbf{m}, k}-\underline{\underline{D}}^{\left(j^{\prime}\right)} \mathbf{r}_{\mathbf{m}^{\prime}, k^{\prime}}\right)\right) .
\end{aligned}
$$

Note that the double sum over the crystallites $j$ and $j^{\prime}$ also applies to the rotation matrices $\underline{D}^{(j)}$ and $\underline{D}^{\left(j^{\prime}\right)}$ in the last line. We now split the scattering function into two terms $G(\mathbf{q})=\overline{\overline{G_{1}}}(\mathbf{q})+G_{2}(\mathbf{q})$ : the first, $G_{1}(\mathbf{q})$, includes only the terms $j=j^{\prime}$ of that sum and thus incorporates scattering of the same crystallite, but not scattering from different crystallites. The second, $G_{2}(\mathbf{q})$, includes only the terms with $j \neq j^{\prime}$, taking into account coherent scattering of two different crystallites.

\subsubsection{Incoherent Part $G_{1}(\mathbf{q})$}

We first consider the case $j=j^{\prime}$ of the sum, i.e. the contribution to the scattering function which is incoherent with respect to different crystallites. Here, the term 
$\exp \left(i \mathbf{q}\left(\mathbf{R}^{(j)}-\mathbf{R}^{\left(j^{\prime}\right)}\right)\right)$ gives 1, and therefore the integral over the crystallite positions $\mathbf{R}^{(j)}$ can be performed and trivially gives 1 due to the normalization of the spatial distribution $\mathcal{P}_{\text {pos }}\left(\mathbf{R}^{(1)}, \ldots, \mathbf{R}^{(N)}\right)$. Furthermore, for each summand $j$ all integrations over the orientations $\underline{\underline{D}}^{(1)}, \ldots, \underline{\underline{D}}^{(N)}$, except $\underline{\underline{D}}^{(j)}$, can be performed and also yield 1 . Therefore, the $N$ terms with $\bar{j}=j^{\prime}$ simplify to:

$$
\begin{aligned}
G_{1}(\mathbf{q}) & =N \int \mathcal{D} \underline{\underline{D}} \sum_{\mathbf{m}, \mathbf{m}^{\prime}=\mathbf{1}}^{\mathbf{M}} \sum_{k, k^{\prime}=1}^{K} F_{k}(\mathbf{q}) F_{k^{\prime}}^{*}(\mathbf{q}) \exp \left(\mathrm { iq } \left(\underline{\underline{D}} \mathbf{r}_{\mathbf{m}, k}-\underline{\left.\left.\underline{D} \mathbf{r}_{\mathbf{m}^{\prime}, k^{\prime}}\right)\right)}\right.\right. \\
& =N \int \mathcal{D} \underline{\underline{D}}\left|\sum_{\mathbf{m}=\mathbf{1}}^{\mathbf{M}} \sum_{p=1}^{P} F_{k}(\mathbf{q}) \exp \left(\mathrm{i}\left(\underline{\underline{D}}^{T} \mathbf{q}\right) \cdot \mathbf{r}_{\mathbf{m}, k}\right)\right|^{2} \\
& =N \int \mathcal{D} \underline{\underline{D}}\left|A\left(\underline{\underline{D}}^{T} \mathbf{q}\right)\right|^{2},
\end{aligned}
$$

where $\underline{\underline{D}}^{T}$ is the transpose of the matrix $\underline{\underline{D}}$ and

$$
A(\mathbf{q}):=\sum_{\mathbf{m}}^{\mathbf{M}} \sum_{k=1}^{K} F_{k}(\mathbf{q}) \exp \left(\mathrm{iq} \cdot \mathbf{r}_{\mathbf{m}, k}\right)
$$

is the unaveraged scattering amplitude of a single unrotated crystallite. Hence, the interpretation of the incoherent part $G_{1}(\mathbf{q})$ is straightforward: each crystallite contributes independently and each with a given orientation. The orientation can be absorbed into the scattering vector $\mathbf{q} \rightarrow \underline{\underline{D}}^{T} \mathbf{q}$, so that the contributions of two crystallites with different orientations are related by a rotation of the scattering vector. In the macroscopic limit, we are allowed to average over all possible orientations and get a sum of $N$ identical terms.

\subsubsection{Coherent Part $G_{2}(\mathbf{q})$}

We now consider the contribution to the scattering intensity from different crystallites, i.e. the case $j \neq j^{\prime}$ in Eq. (4.14). In analogy to the above calculation, all integrations over the orientations $\underline{\underline{D}}^{(1)}, \ldots, \underline{\underline{D}}^{(N)}$ can be performed except for $\underline{\underline{D}}^{(j)}$ and $\underline{\underline{D}}^{\left(j^{\prime}\right)}$ :

$$
\begin{aligned}
G_{2}(\mathbf{q})= & \int \mathrm{d} \mathbf{R}^{(1)} \cdots \mathrm{d} \mathbf{R}^{(N)} \mathcal{P}_{\operatorname{pos}}\left(\mathbf{R}^{(1)}, \ldots, \mathbf{R}^{(N)}\right) \\
& \times \sum_{j \neq j^{\prime}} \exp \left(\operatorname{iq}\left(\mathbf{R}^{(j)}-\mathbf{R}^{\left(j^{\prime}\right)}\right)\right) \\
& \times \int \mathcal{D} \underline{\underline{D}} \mathcal{D} \underline{\underline{D}}{ }_{\mathbf{m}, \mathbf{m}^{\prime}=\mathbf{1}} \sum_{k, k^{\prime}=1}^{K} F_{k}(\mathbf{q}) F_{k^{\prime}}^{*}(\mathbf{q}) \exp \left(\mathrm{iq}\left(\underline{\underline{D} \mathbf{r}_{\mathbf{m}, k}}-\underline{\underline{D^{\prime}}} \mathbf{r}_{\mathbf{m}^{\prime}, k^{\prime}}\right)\right)
\end{aligned}
$$


where we have used that the angular distribution is the same for all crystallites. We introduce the structure factor of the crystallite positions:

$$
\begin{aligned}
S(\mathbf{q})=\frac{1}{N}\left\langle\sum_{j, j^{\prime}=1}^{N}\right. & \left.\exp \left(i \mathbf{q}\left(\mathbf{R}^{(j)}-\mathbf{R}^{\left(j^{\prime}\right)}\right)\right)\right\rangle \\
=1+\frac{1}{N} \int & \mathrm{d} \mathbf{R}^{(1)} \cdots \mathrm{d} \mathbf{R}^{(N)} \mathcal{P}_{\operatorname{pos}}\left(\mathbf{R}^{(1)}, \ldots, \mathbf{R}^{(N)}\right) \\
& \times \sum_{j \neq j^{\prime}} \exp \left(\mathbf{i q}\left(\mathbf{R}^{(j)}-\mathbf{R}^{\left(j^{\prime}\right)}\right)\right)
\end{aligned}
$$

and observe that the two upper lines in $(4.17)$ are just $N(S(\mathbf{q})-1)$. This simplifies $G_{2}(\mathbf{q})$ to:

$$
\begin{aligned}
G_{2}(\mathbf{q}) & =N(S(\mathbf{q})-1)\left|\int \mathcal{D} \underline{\underline{D}} \sum_{\mathbf{m}=\mathbf{1}}^{\mathbf{M}} \sum_{k=1}^{K} F_{k}(\mathbf{q}) \exp \left(\mathrm{i}\left(\underline{\underline{D}}^{T} \mathbf{q}\right) \cdot \mathbf{r}_{\mathbf{m}, k}\right)\right|^{2} \\
& =N(S(\mathbf{q})-1)\left|\int \mathcal{D} \underline{\underline{D}} A\left(\underline{\underline{D}}^{T} \mathbf{q}\right)\right|^{2}
\end{aligned}
$$

Again the interpretation is straightforward: for coherent scattering the amplitudes of individual crystallites with different orientations add up, as expressed by $\int \mathcal{D} \underline{\underline{D}} A\left(\underline{\underline{D}}^{T} \mathbf{q}\right)$. Spatial correlations of the centers of the crystallites are accounted for by the structure function $S(\mathbf{q})$.

The total scattering function

$$
\frac{G(\mathbf{q})}{N}=\int \mathcal{D} \underline{\underline{D}}\left|A\left(\underline{\underline{D}}^{T} \mathbf{q}\right)\right|^{2}+(S(\mathbf{q})-1)\left|\int \mathcal{D} \underline{\underline{D}} A\left(\underline{\underline{D}}^{T} \mathbf{q}\right)\right|^{2}
$$

is reduced to the scattering amplitude of a single crystallite $A(\mathbf{q})$, which we compute next. Note that if the angular spread of the crystallites can be neglected, in other words all crystallites are approximately aligned, then the above expression reduces to $G(\mathbf{q})=N S(\mathbf{q})|A(\mathbf{q})|^{2}$, as would be expected.

If there is thermal motion of the atoms around their equilibrium positions due to finite temperature, the intensity of the scattering function $G(\mathbf{q})$ is weakened for larger $q$-values [e.g. Willis \& Pryor, 1975]. The resulting scattering function has to be multiplied with the Debye-Waller factor

$$
G_{\mathrm{DW}}(\mathbf{q})=G(\mathbf{q}) \cdot \exp \left(-\mathbf{q}^{2}\left\langle u^{2}\right\rangle\right)
$$

where $\left\langle u^{2}\right\rangle$ is the mean square displacement of the atoms in any direction.

\subsubsection{Scattering Amplitude of a Single Crystallite}

The calculation of the scattering amplitude of a single crystallite $A(\mathbf{q})$ (defined in Eq. (4.16)) follows standard procedures. We substitute the atomic positions of 

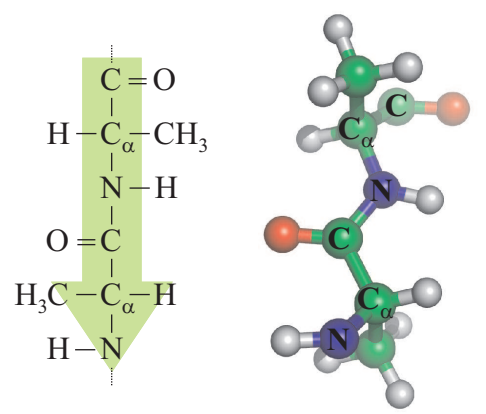

Figure 4.7: Chemical structure (left) and conformation (right) of a polyalanine strand. Two alanines are shown. The $\mathrm{CH}_{3}$ group is characteristic for the alanine amino acid and is bound to the so called $\mathrm{C}_{\alpha}$ atom. The arrow indicates the direction $\mathrm{C} \rightarrow \mathrm{C}_{\alpha} \rightarrow \mathrm{N}$ along the backbone.

Sec. 4.2.2 and note that the sums over $m_{x}, m_{y}$ and $m_{z}$ are geometric progressions which can easily be performed. As seen in Appendix E.3, the scattering amplitude becomes:

$$
A(\mathbf{q})=L_{M_{x}}\left(\mathbf{q} \mathbf{a}_{x}\right) L_{M_{y}}\left(\mathbf{q} \mathbf{a}_{y}\right) L_{M_{z}}\left(\mathbf{q} \mathbf{a}_{z}\right) \sum_{k=1}^{K} F_{k}(\mathbf{q}) \exp \left(\mathbf{i q} \mathbf{r}_{k}\right)
$$

Here $L_{M_{\nu}}\left(\mathbf{q} \mathbf{a}_{\nu}\right)=\frac{\sin \left(\mathbf{q} \mathbf{a}_{\nu} M_{\nu} / 2\right)}{\sin \left(\mathbf{q} \mathbf{a}_{\nu} / 2\right)}$ is the well-known Laue function, which has an extreme value when its argument $\mathbf{q} \mathbf{a}_{\nu}$ is a multiple of $2 \pi$. If $M_{\nu}$ is finite, the peak has a finite width and gets wider with a decreasing number of repetitions $M_{\nu}$.

It is noteworthy that the extremum of the magnitude of the scattering amplitude $A(\mathbf{q})$ may be shifted to a slightly different position, if the form factor of the unit cell $\sum_{k=1}^{K} F_{k}(\mathbf{q}) \exp \left(\mathbf{i q r}_{k}\right)$ has a non-vanishing gradient at that position. In this case, the resultant peak may be shifted by a value of the order of its peak width. Consequently, care has to be taken when determining lattice constants from experimental peak positions.

\subsection{Atomic Configuration of the Unit Cell}

The computation of the scattering function $G(\mathbf{q})$ requires the atomic configuration $\left\{\mathbf{r}_{k}\right\}_{k=1}^{K}$ of the unit cell, which we discuss next.

\subsubsection{Unshifted Unit Cells}

It is known that the crystallites are composed of polyalanine strands [see Arnott et al., 1967; Grubb \& Jelinski, 1997]. In Fig. 4.7 two alanine amino acids of this strand are shown. Its conformation, shown on the right, is well established and was modeled with Yasara [Meling, 2006]. There are two constraints for the strand: firstly, the subsequent alanines in the strand must have the same orientation so that the strand does not have a "twist" and can produce periodic structures. And 
secondly, the distance between adjacent alanines has to match the size of the unit cell. These two constraints allow for a unique choice of the two degrees of freedom, namely the Ramachandran angles [Ramakrishnan \& Ramachandran, 1965] $\Phi$ and $\Psi$, which are the dihedral angles for the bonds $\mathrm{C}_{\alpha}-\mathrm{N}$ and $\mathrm{C}_{\alpha}-\mathrm{C}$, respectively.

Many of the described polyalanine strands placed side by side form a stable crystalline configuration, the $\beta$-pleated-sheet. We assume an orthorhombic unit cell $^{1}$, consisting of four alanine strands, as illustrated in Fig. 4.8. Thus one unit cell contains 8 alanine amino acids. Furthermore, we define the spatial directions in the usual way [e.g. Warwicker, 1960]:

$x$ : Direction of the $\mathrm{CH}_{3}$-groups and Van-der-Waals interactions between sheets lying upon each other.

$y$ : Direction of the hydrogen bonds between the O-atom of one strand and the $\mathrm{H}$-atom of the neighboring strand.

$z$ : Direction of the covalent bonds along the backbone.

Accordingly, $\mathbf{a}_{x}, \mathbf{a}_{y}$ and $\mathbf{a}_{z}$ are the principal vectors pointing in these directions and $a_{x}, a_{y}, a_{z}$ their magnitudes. Note furthermore that due to symmetry the distance between the strands has to be $a_{x} / 2$ in the $x$-direction and $a_{y} / 2$ in the $y$-direction.

In general, one has to distinguish between the parallel and antiparallel structure. In the parallel structure, the direction of the atom sequence $\mathrm{C} \rightarrow \mathrm{C}_{\alpha} \rightarrow \mathrm{N}$ in the strand's backbone is the same for all strands (left side of Fig. 4.8). For the antiparallel structure this direction alternates along the $y$-axis (right side of Fig. 4.8). Both will be considered in the following analysis.

\footnotetext{
${ }^{1}$ Note that the assumption of an orthorhombic unit cell is restrictive. In a more general approach one can give up this assumption and use a smaller unit cell, allowing for different shifts. However, the best fit to the data is obtained for a shift which can also be achieved with an orthorhombic unit cell. For the sake of clarity, we stick to the established unit cell notations and indexing of the reflexes here.
} 

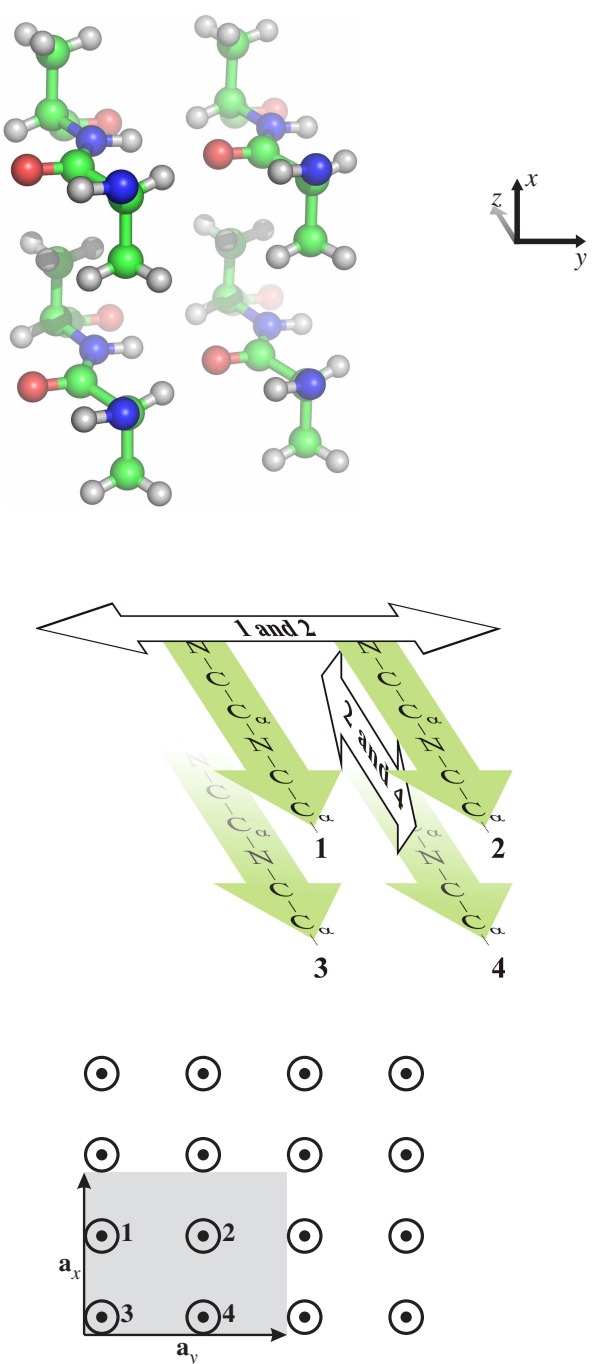
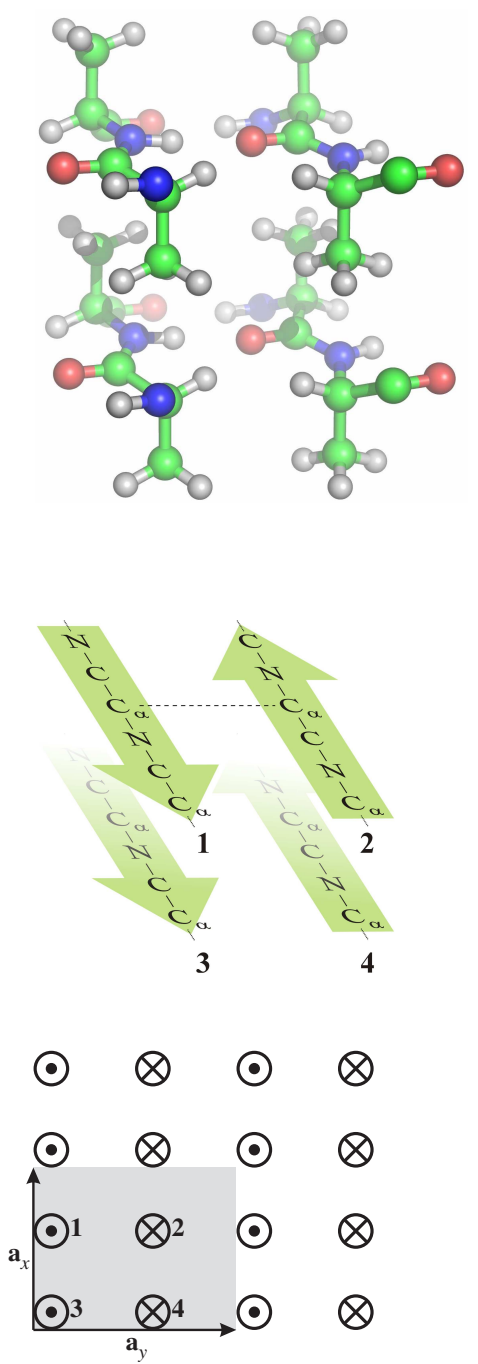

Figure 4.8: Illustration of one unit cell, containing four neighboring polyalanine strands. Top: Configuration of the atoms. Center: Schematic view displaying only the backbone atoms. The white arrows illustrate two possible adjustments of the structure, which are to be optimized to match the experimental scattering image. Bottom: Simplified schematic view along the backbone axis $\mathbf{a}_{z}$, where $\odot$ indicates an arrow pointing towards the reader and $\otimes$ pointing away from the reader. Here, the unit cell is shown as a gray rectangle. In each case, a parallel structure is shown on the left side, and an antiparallel structure on the right side. The illustration shows an unshifted configuration, which means: the $\mathrm{C}_{\alpha}$ atoms of neighboring strands are aligned and have no shift in $z$-direction (indicated by the dashed line in the middle right image). Additionally, the strands are exactly aligned in the $x$ - and $y$-direction. 


\subsubsection{Possible Shifts inside the Unit Cell}

The scattering intensity is not only sensitive to the conformation of the polyalanine strands, but also to the distance and orientation of different strands relative to each other. Besides the question of parallel or antiparallel structure, the four strands can also be shifted with respect to each other. In principle there are four ways to displace them (see Fig. 4.8):

- Shifting strands 1 and 2 in the $y$-direction by a value $\Delta y_{12}$. This displacement is performed in Fig. 4.9.

- Shifting strands 1 and 2 in the $z$-direction by a value $\Delta z_{12}$. Because of the $\mathrm{CH}_{3}$-groups extending into the layers above and below (as seen in Fig. 4.8, top panel), a displacement like this is only possible if those two strands have a shift in the $y$-direction of $\Delta y_{12} \approx \pm \mathbf{a}_{y} / 4$ as well. In this case, the $\mathrm{CH}_{3}$-groups can pass each other without overlapping.

- Shifting strands 2 and 4 in the $z$-direction by a value $\Delta z_{24}$. This shift was originally suggested by Arnott et al. [1967].

- Shifting strands 2 and 4 in the $x$-direction. However this displacement would have a high energy cost, because it would break the hydrogen bonds between the $\mathrm{H}$ - and $\mathrm{O}$-atoms of neighboring strands. Since, moreover, no reasonable result could be achieved performing such shifts, it will not be included in the discussion any further.

Note that shifting strands 1 and 2 in the $x$-direction, or strands 2 and 4 in the $y$-direction, is associated with resizing the unit cell in the $x$ - or $y$-direction, respectively.
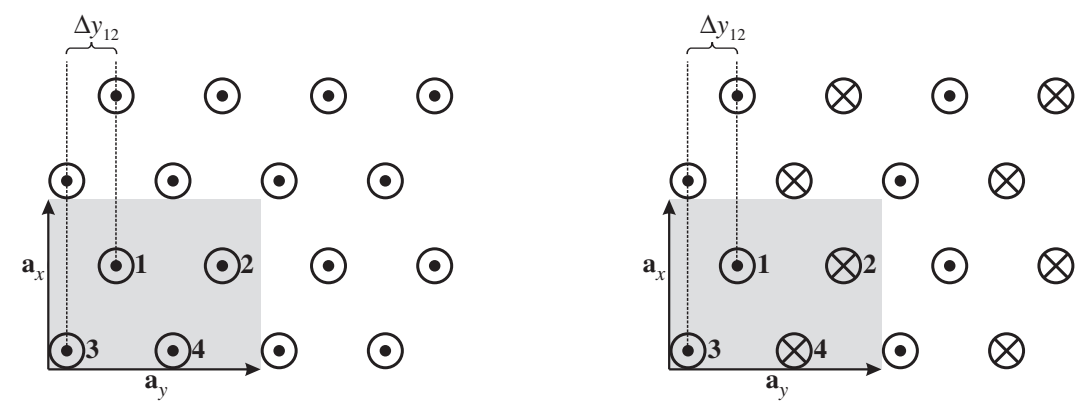

Figure 4.9: Illustration of the unit cell, as at the bottom of Fig. 4.8, but with the conducted shift $\Delta y_{12}=a_{y} / 4$ of strands 1 and 2 the in $y$-direction.

\subsubsection{Variations between Crystallites}

For real systems, the composition of the crystallite is certainly not fixed but may vary from crystallite to crystallite. These variations can easily be included into 
our model by introducing a probability distribution $P\left(\left\{r_{\mathbf{m}, k}\right\}\right)$ for the crystallite configuration $\left\{r_{\mathbf{m}, k}\right\}$, with the normalization $\sum_{\left\{r_{\mathbf{m}, k}\right\}} P\left(\left\{r_{\mathbf{m}, k}\right\}\right)=1$. Note that the probability distribution may be chosen such that the crystallite size $\left(M_{x}, M_{y}, M_{z}\right)$ is variable and also that the unit cells may have different types and different numbers of atoms. The calculation is performed completely analogously to the orientational distribution. The scattering function becomes

$$
\begin{aligned}
\frac{G(\mathbf{q})}{N}= & \sum_{\left\{r_{\mathbf{m}, k}\right\}} P\left(\left\{r_{\mathbf{m}, k}\right\}\right) \int \mathcal{D} \underline{\underline{D}}\left|A\left(\underline{\underline{D}}^{T} \mathbf{q}\right)\right|^{2} \\
& +(S(\mathbf{q})-1)\left|\sum_{\left\{r_{\mathbf{m}, k}\right\}} P\left(\left\{r_{\mathbf{m}, k}\right\}\right) \int \mathcal{D} \underline{\underline{D}} A\left(\underline{\underline{D}}^{T} \mathbf{q}\right)\right|^{2}
\end{aligned}
$$

Later (Sec. 4.5.2), we investigate the effect of variable crystallite sizes as well as the influence of small fractions of glycine inside the crystallites. The effect of the coherent scattering term on the bottom line of Eq. (4.23) is analyzed in Appendix E.2.

\subsection{Results}

\subsubsection{Experimental Scattering Function}

Two types of samples have been investigated: fiber bundles and single fiber preparations. Single fibers demand a highly collimated and brilliant beam, but are better defined in orientation and are amenable to simultaneous strain-stress measurements.

An oriented bundle of major ampullate silk (MAS) of Nephila Clavipes was measured at the D4 bending magnet of HASYLAB/DESY in Hamburg. The fibers were reeled on a steel holder and oriented horizontally in the beam. The number of threads was estimated at 400-600. Photon energy was set to $E=10.9 \mathrm{keV}$ by a $\mathrm{Ge}(111)$ crystal monochromator, located behind a mirror to suppress higher harmonics. Data was collected by a CCD X-ray camera (SMART Apax, AXS Bruker). Illumination was triggered by a fast shutter. The momentum transfer was calibrated by a standard (corrundum) and raw data was corrected by an empty image (background subtracted).

The single fiber experiments were performed at the microfocus beamline ID13 at ESRF, Grenoble [Riekel \& Davies, 2005]. A $12.7 \mathrm{keV}$ X-ray beam was focused with a pair of short focal length Kirkpatrick Baez mirrors [Kirkpatrick \& Baez, 1948] to a $7 \mu \mathrm{m}$ spot on the sample. This focusing scheme provides a sufficient flux density $\left(6.8 \cdot 10^{15} \mathrm{cps} / \mathrm{mm}^{2}\right)$ to obtain diffraction patterns from single dragline fibers. The single fiber diffraction patterns were recorded with a CCD detector positioned $131 \mathrm{~mm}$ behind the sample (Mar 165 detector, Mar USA, Evanston, IL). One of the beamline's custom-made lead beamstops (approx. $300 \mu \mathrm{m}$ diameter) was used to block the intense primary beam. The raw data was treated as follows: (i) both the image and the background (empty beam) were corrected by dark current, and (ii) 
the background was subtracted from the image. The peaks which are significantly broadened by the small crystallite size (see below) can then be indexed by the orthorhombic lattice described above.

Typical scattering distributions for both types of sample preparations are shown in Fig. 4.10, as a function of parallel and vertical momentum transfer. More details on experimental procedures and on the sample preparation by forced silking can be found in [Glišović \& Salditt, 2007; Glišović et al., 2008].

\subsubsection{Scattering Function from the Model}

It is our aim to determine those crystallites' parameters which best match the experimental result. The free parameters of our model are the three shifts $\Delta y_{12}$, $\Delta z_{12}$ and $\Delta z_{24}$, the unit cell dimensions $a_{x}, a_{y}$ and $a_{z}$, the crystallite size in the three directions $M_{x}, M_{y}$ and $M_{z}$, as well as $\theta_{0}$, the tilting angle of the crystallites away from the fiber axis.

These parameters affect the scattering intensity in different ways, allowing us to at least partially separate the effects of different parameters. The crystallite size $\left(M_{x}, M_{y}, M_{z}\right)$ determines the peak widths, whereas the length of the principal vectors $\mathbf{a}_{x}, \mathbf{a}_{y}$ and $\mathbf{a}_{z}$ determine the peak position. (We have to keep in mind, however, that the peak position can differ from the extremal values of the Laue functions, as explained in Sec. 4.3.3.) The shifts $\Delta y_{12}, \Delta z_{12}$ and $\Delta z_{24}$, as described in Sec. (4.4.2), affect the relative peak intensities via the form factors of the unit cell $\sum_{k=1}^{K} F_{k}(\mathbf{q}) \exp \left(\mathbf{i q r}_{k}\right)$. Finally, the parameter $\theta_{0}$ is responsible for the peak widths in the azimuthal direction on the scattering image.

From Eq. (4.22) it is clear that the $z$-components of the atom positions $\left\{\mathbf{r}_{k}\right\}_{k=1}^{K}$ are irrelevant for the scattering amplitude $A(\mathbf{q})$ in the $x y$-plane, i.e. for a zero $z$ component of $\mathbf{q}$. Therefore, parameters affecting only the $z$-components - especially the mentioned shifts in the $z$-direction - will not influence the intensity profile of $G(\mathbf{q})$ in the $x y$-plane. ${ }^{2}$ Analogously, the scattering profile in the $z$-direction is independent of parameters influencing the $x$ - and $y$-directions. Consequently, the sections of the scattering profile along and perpendicular to the fiber axis can separately be matched to subsets of the parameters. The intensity profile off the $z$ and $x y$-axes, taking into account all dimensions of the crystallite, can be seen as a consistency check for the found parameters.

The experimental scattering data clearly reveal a (002) peak, Fig. 4.10. This peak is allowed by symmetry; however, it is extremely weak in the antiparallel structure suggested by Marsh et al. [1955b] and shown in Fig. 4.11. The reason is that the electron density within the unit cell projected along the $z$-axis is almost uniform, varying by approximately $10 \%$. We therefore consider two alternative

\footnotetext{
${ }^{2}$ In principle there can be an influence because of the $\theta$-tilt of the crystallites with respect to the fiber axis (see Sec. 4.2.3 and Fig. 4.5). However, the scattering amplitude $A(\mathbf{q})$ shows a discrete peak structure and, for small $\theta$-rotations, the out-of-plane reflections (which are influenced by the $z$-components) are too far away from the $x y$-plane to have an impact on the in-plane intensity profile.
} 
mechanisms generalizing the classical model (Marsh et al.) of the antiparallel unit cell. By both mechanisms, the intensity of the (002) peak will increase in agreement with the experiment:
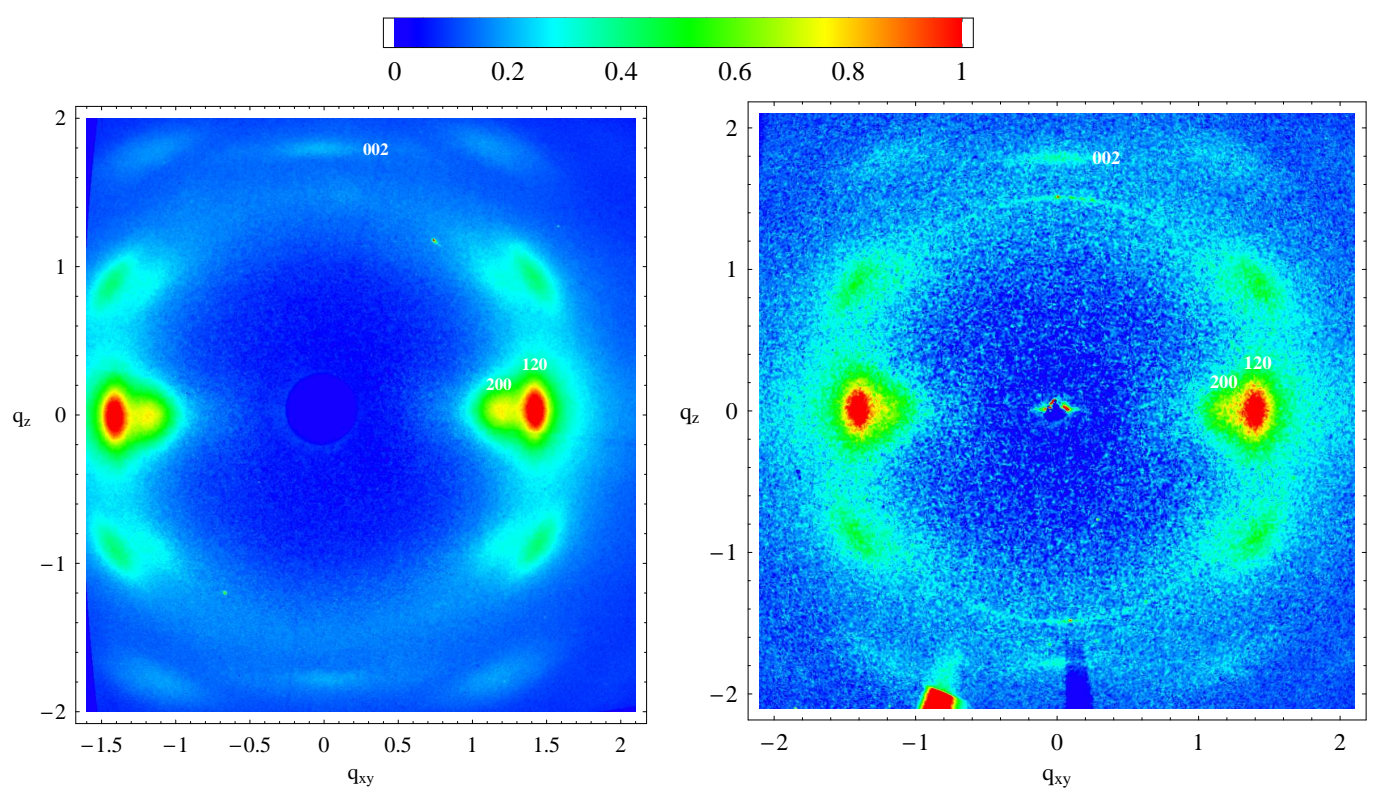

Figure 4.10: Scattering images of spider silk. The experimental scattering image of spider silk from Nephila clavipes is shown, both for bundle measurements (left) and single fiber diffraction (right). The fiber axis runs vertically. At the top of the figure the color-bar shows scattering intensities, which are normalized by the intensity of the (120) peak. By courtesy of Tim Salditt.

a) the shift of strands $\mathbf{2}$ and $\mathbf{4}$ in the $z$-direction, i.e. a non-zero $\Delta z_{24}$-shift or

b) structural disorder affecting the almost uniform electron density.

We first discuss case a). The uniform electron density is disturbed by a shift $\Delta z_{24} \neq$ 0 . The intensity of the (002) reflection grows accordingly with an increasing shift $\Delta z_{24}$. Adjusting the $\Delta z_{24}$-shift yields results consistent with experiments. Table 4.1 shows the results for the parameters of the model, obtained from optimizing the agreement between the calculated scattering function and the experimental one. For comparison the set of parameters for both the parallel and the antiparallel structure are presented. On the basis of the experimental data, one can not discriminate between the parallel and the antiparallel structure.

The scattering intensities, as calculated with these values, are shown in Fig. 4.12. The crystallites are randomly rotated about the fiber axis so that on average the system is invariant under rotations around the fiber axis. Consequently, the scattering image also has rotational symmetry about the $z$-axis and the $q_{x^{-}}$and $q_{y^{-}}$-axis are indistinguishable and denoted by $q_{x y}$. A section along the $q_{x y}$-axis is shown in Fig. 4.13, top panel. The mismatch for $q$-values slightly larger than the (120) peak 


\begin{tabular}{|c|c|c|c|c|c|}
\hline \multirow{2}{*}{$\frac{\text { structure of }}{\text { alignment }}$} & $\begin{array}{l}\text { presente } \\
\text { Nephila }\end{array}$ & $\begin{array}{l}\text { calculation } \\
\text { lavipes }\end{array}$ & \multirow{2}{*}{$\begin{array}{l}\text { Warwicker } \\
{[1954]} \\
\text { Bombyx mori } \\
\text { anti-parallel } \\
\end{array}$} & \multirow{2}{*}{$\begin{array}{l}\text { Marsh et al. } \\
{[1955 b]} \\
\text { Tussah Silk } \\
\text { anti-parallel }\end{array}$} & \multirow{2}{*}{$\begin{array}{l}\text { Arnott et al. } \\
\text { [1967] } \\
\text { poly-L-alanine } \\
\text { anti-parallel }\end{array}$} \\
\hline & parallel & anti-parallel & & & \\
\hline $\bar{a} a_{x}$ & $10.0 \AA$ & $10.0 \AA$ & $10.6 \AA$ & $10.6 \AA$ & $10.535 \AA$ \\
\hline$a_{y}$ & $9.3 \AA$ & $9.3 \AA$ & $9.44 \AA$ & $9.44 \AA$ & $9.468 \AA$ \\
\hline$a_{z}$ & $6.95 \AA$ & $6.95 \AA$ & $6.95 \AA$ & $6.95 \AA$ & $6.89 \AA$ \\
\hline$M_{x}$ & $1.5^{(*)}$ & $1.5^{(*)}$ & - & - & - \\
\hline$M_{y}$ & 5 & 5 & - & - & - \\
\hline$M_{z}$ & 9 & 9 & - & - & - \\
\hline$\Delta y_{12}$ & $a_{y} / 4$ & $a_{y} / 4$ & 0 & $a_{y} / 4$ & $\pm a_{y} / 4^{(* *)}$ \\
\hline$\Delta z_{12}$ & 0 & 0 & 0 & 0 & \\
\hline$\Delta z_{24}$ & 0 & $-a_{z} / 6$ & 0 & 0 & $-a_{z} / 10$ \\
\hline $\begin{array}{l}\theta_{0} \\
\left\langle u^{2}\right\rangle\end{array}$ & $\begin{array}{l}7.5^{\circ} \\
0.1 \AA^{2}\end{array}$ & $\begin{array}{l}7.5^{\circ} \\
0.1 \AA^{2}\end{array}$ & - & & $\begin{array}{l}- \\
- \\
-\end{array}$ \\
\hline
\end{tabular}

Table 4.1: Summary of parameters. The left two columns show the best match between experimental and calculated scattering functions. For $a_{z}=6.95 \AA$, the resulting Ramachandran angles are $\Phi=-139.0^{\circ}$ and $\Psi=136.9^{\circ} .\left\langle u^{2}\right\rangle$ was used for the Debye-Waller factor in Eq. (4.21). The three right columns compare our obtained parameters with the literature.

$(*)$ Note that each unit cell contains two layers of alanine-strands in $x$-direction. Therefore $M_{x}=1.5$ corresponds to three layers of $\beta$-sheets in a single crystallite.

(**) Statistical model: A layer is shifted by a value $+a_{y} / 4$ or $-a_{y} / 4$ with respect to the previous layer, where + and - are equally likely.

is plausible, because in this region the amorphous matrix contributes noticeably to the experimental scattering intensity, but has been neglected in the model. The oscillations of the calculated scattering image for low $q$-values are side maxima, which are suppressed by fluctuations in the crystallite sizes (see Sec. 4.4.3). The corresponding scattering intensities are shown in Fig. 4.13, and the full scattering image is in Fig. E.2 (left) in Appendix E.2 (page 189). Clearly, the side maxima have been flattened out.

We now discuss an alternative mechanism to generate a stronger (002) peak by introduction of disorder into the amino acid composition of the unit cell (case b). Polyalanine as a model for the crystallites in spider silk is an over-simplification, since the amino acid sequence hardly allows for a pure polyalanine crystallite. Instead, we expect that other residues must be incorporated into the crystallite even if energetically less favorable to compromise the given sequence. In particular, it is highly likely that glycine amino acids are also embedded in the crystallites [Marsh et al., 1955a]. This can be easily implemented by replacing randomly selected alanine amino acids of the crystallites with glycine (see Sec. 4.4.3). It is found that the intensity of the (002) peak increases with the fraction of substituted alanines. In Fig. 4.11 we compare the original Marsh-structure (without glycine) to a structure with the same parameters, but with alanine randomly replaced with glycine with a 
probability $p_{\mathrm{gl}}=0.375$. The random substitution has clearly produced an intensity of the (002) peak comparable to the experiment.
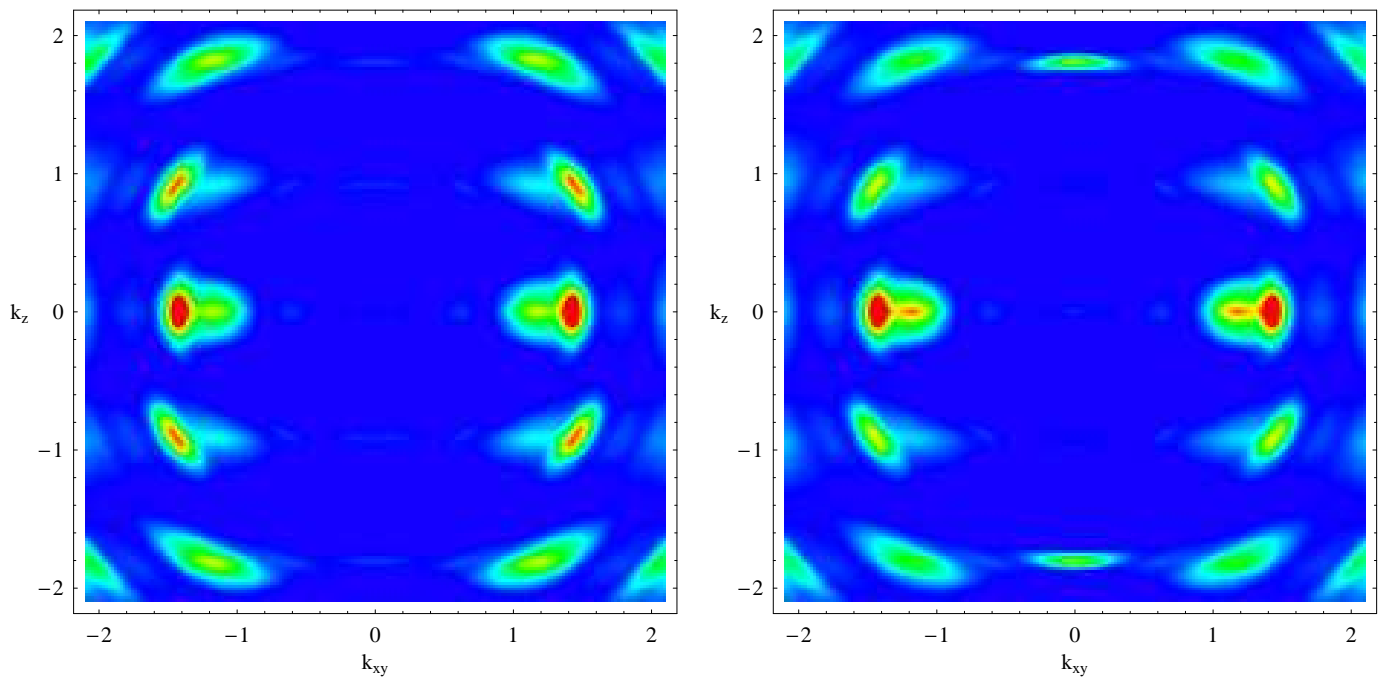

Figure 4.11: Calculated scattering images of the structure proposed by Marsh et al. [1955b]. The unit cell size is $\left(a_{x}, a_{y}, a_{z}\right)=(10.6,9.44,6.95) \AA$, and we used the crystallite size $\left(M_{x}, M_{y}, M_{z}\right)=(1.5,6,9)$. Left: The crystallites are made purely of alanine amino acids. Right: The crystallites' alanine amino acids are replaced with glycine with a probability $p_{\mathrm{gl}}=0.375$.
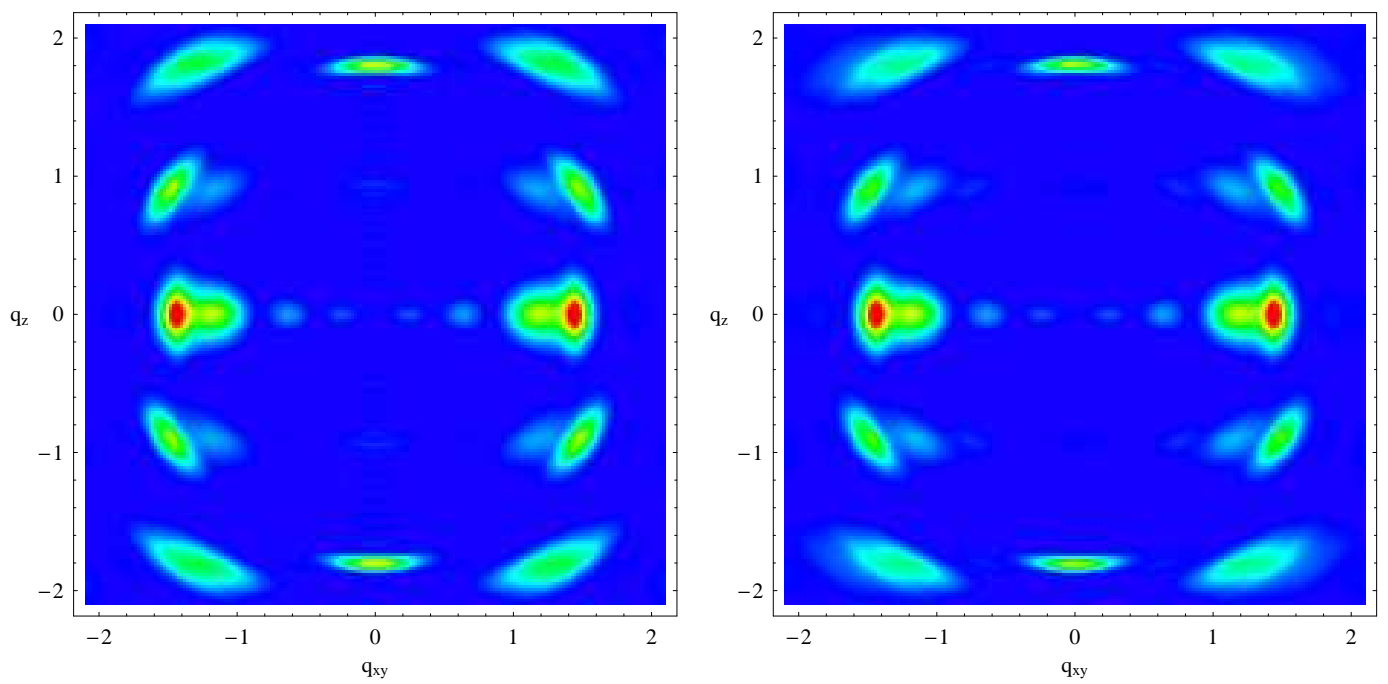

Figure 4.12: Scattering images, as calculated from Eq. (4.20), for the parallel structure on the left side and the antiparallel structure on the right side. 

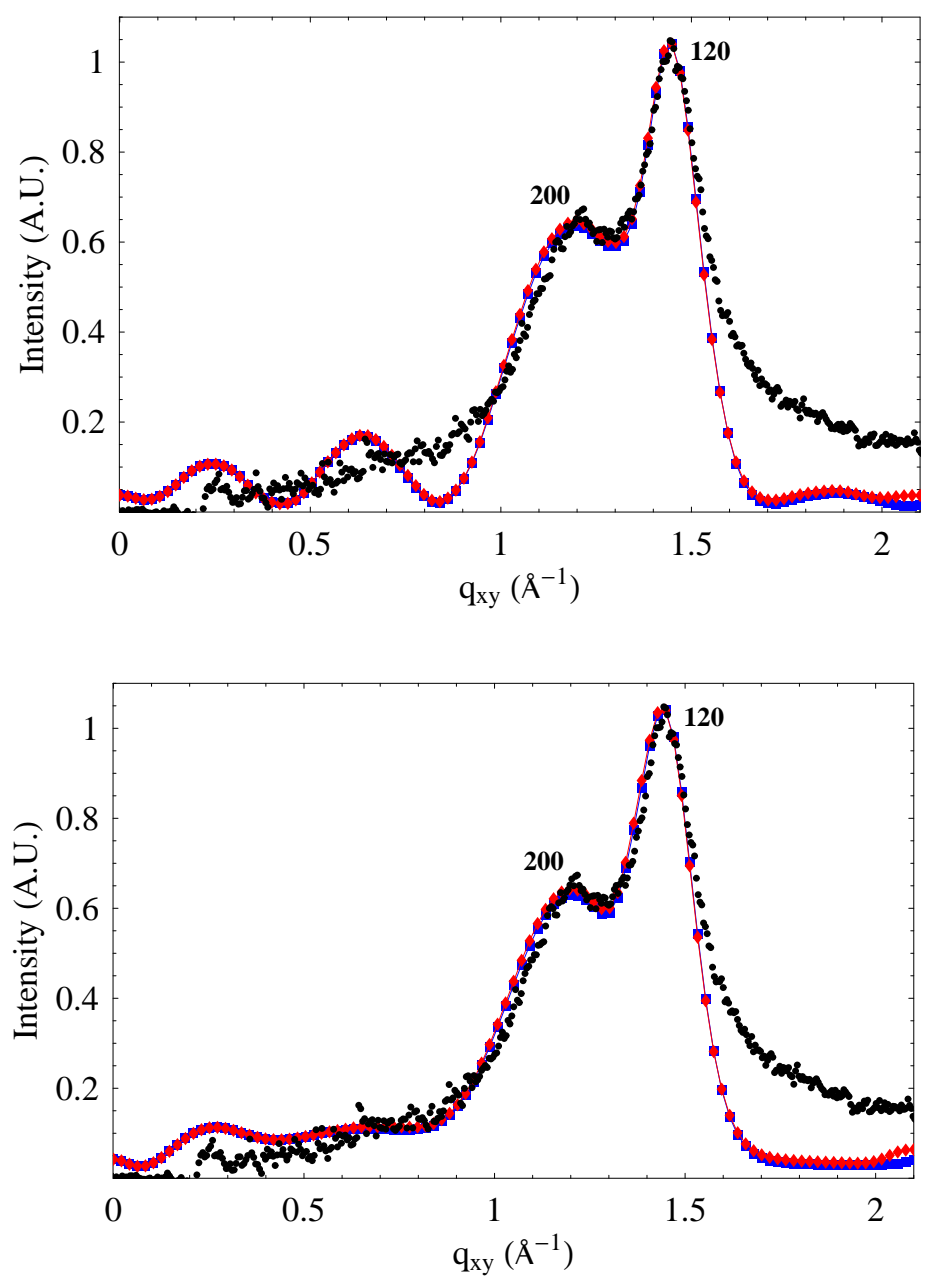

Figure 4.13: Top: Comparison of a section of the experimental $(\bullet)$ and the calculated scattering intensity ( - parallel, $\uparrow$ antiparallel). Sections of the profiles in Fig. 4.12 along the $q_{x y}$-axis, i.e. the scattering profile perpendicular to the fiber axis, are shown. Bottom: As top, but with a Gaussian distribution (rounded to integers) of the crystallite sizes $M_{x}, M_{y}$ and $M_{z}$. The widths are $\Delta M_{x}=2, \Delta M_{y}=0.75$ and $\Delta M_{z}=3$ respectively. 


\subsection{Conclusions}

We have developed a microscopic model of the structure of spider silk. The main ingredients of the model are the following:

a) many small crystallites are distributed randomly in an amorphous matrix,

b) the orientation of the crystallites fluctuate with a preferential alignment along the fiber axis,

c) each crystallite is composed typically of $5 \times 2 \times 9$ unit cells,

d) each unit cell contains four alanine strands shifted with respect to each other. Disorder can be generated by randomly replacing alanine with glycine.

We have computed the scattering intensity of our model and compared it to wide angle X-ray scattering data of spider silk from Nephila clavipes. Possible inter-crystallite correlations are unimportant given the measured orientational distribution. In other words, even if significant center-of-mass correlations between crystallites were present, the orientational distribution would suppress interference effects, with the exception of the (002) peak, which is least sensitive to orientational disorder. The contribution of coherent scattering is discussed in detail in Appendix E.2.

A homogeneous electron density background is a necessary feature of the scattering model. Calculation of the crystal structure factor in vacuum does not only lead to an incorrect overall scaling prefactor (which is important if absolute scattering intensities are measured), but also leads to a scattering intensity distribution with artifacts at small and intermediate momentum transfer.

The comparison between model and data fixes the parameters of the unit cell and the crystallite for the two possible cases, the parallel and the antiparallel structure, respectively, as shown in Table 4.1. The two models with parallel and antiparallel alignment of the alanine strands yield comparable agreement with the experimental data. Furthermore a more refined model, where alanine is randomly replaced with glycine, gives reasonable results. Hence we cannot rule out one of these structures.

Our model is similar to the model of the poly-L-alanine of Arnott et al. [1967]. Their model does incorporate a $\Delta z_{24}$-shift. However, our structure shows a better agreement with the experimentally measured scattering function using a value of $\Delta z_{24}=-a_{z} / 6$.

While we have concentrated here on the wide angle scattering reflecting the crystalline structure on the molecular scale, the same model can be used for small angle scattering to analyze the short range order between crystallites in the presence of orientational and positional fluctuations. In particular, the model can describe the entire range of momentum transfer and the transition from wide angle scattering (WAXS) to small angle scattering (SAXS). Note that WAXS is usually described 
only in the single object approximation, neglecting inter-particle correlations. Contrarily, SAXS is mostly described in continuum models without crystalline parameters. Here, both are treated by the same approach, which is a significant advantage for systems where the length scales are not decoupled. 
Chapter 5

\title{
Aggregation and Gelation in Wet Granular Materials
}

\author{
Contents \\ 5.1 Introduction $\ldots \ldots \ldots \ldots \ldots \ldots \ldots$ \\ 5.2 Models . . . . . . . . . . . . . . . . . 83 \\ 5.3 Cooling Dynamics $\ldots \ldots \ldots \ldots \ldots$ \\ 5.3.1 Early Stage of Cooling . . . . . . . . . . . . . . . . . . 87 \\ 5.3 .2 Late Stage of Cooling . . . . . . . . . . . . . . . . . 90 \\ 5.3 .3 Velocity Distribution . . . . . . . . . . . . . . . . . 95 \\ 5.3.4 Partitioning of the Energy into Translational, Rotational and \\ Internal Degrees of Freedom . . . . . . . . . . . . . 98
}

5.4 Aggregation . . . . . . . . . . . . . . . . . 101

5.4 .1 Self-Similar Growth . . . . . . . . . . . . . . . . . 103

5.4.1.1 Fractal Dimension of the Aggregates . . . . . . . . . 103

5.4.1.2 Cluster Mass Distribution . . . . . . . . . . . . . . . 105

5.4.1.3 Number of Clusters and Mean Cluster Mass . . . . . 106

5.4.2 Properties of the Asymptotic Cluster . . . . . . . . . . . . . . . 107

5.4.2.1 Fractal Dimension from Radius of Gyration . . . . . . 108

5.4.2.2 Fractal Dimension from Box Counting Algorithm . . 110

5.4.2.3 Pair Correlation Function and Correlation Dimension 114

5.4.2.4 Coordination Number . . . . . . . . . . . . . . . . . 118

5.4.2.5 Inertia Tensor and Spatial Extension . . . . . . . . 122

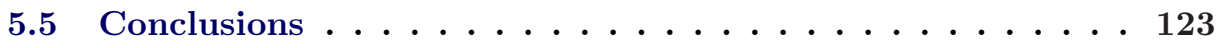

\subsection{Introduction}

Granular materials are systems of macroscopic particles, which are typically characterized by two properties: They interact only on contact and their interactions are dissipative. In spite of their simple interaction, they are of high importance for both science and for industry: On the one hand, because granular systems yield a broad range of interesting nonequilibrium phenomena [Brilliantov \& Pöschel, 2004; 
Kudrolli, 2004; Umbanhowar et al., 1996] and have been considered as model systems for structure formation on various length scales, including the formation of planetesimals from interstellar dust and the formation of planets and stars from accretion disks [Blum et al., 2000; Bridges et al., 1984]. And on the other hand, storage and handling of bulk solids is among the most significant tasks in industrial technology and still poses a large number of unsolved problems [Jaeger et al., 1996; Duran, 2000]; in addition granular materials are the second-most manipulated material by mankind (the first one being water) [de Gennes, 1999].

Most studies discuss dry granular systems, where the dissipative contact interaction consists in the loss of a certain fraction of the kinetic energy in every impact [Duran, 2000; Brilliantov \& Pöschel, 2004, and references therein]. Adding a small amount of liquid to the granular system changes its properties dramatically: while dry sand can flow through one's fingers similarly to a liquid, wet sand has properties of a plastic solid, even suitable to build structures like sand castles. This difference in the macroscopic behavior is reflected in the corresponding difference in particle interactions [Herminghaus, 2005]:

Wet granular particles are covered by a thin liquid film. When two wet particles come into contact, the films merge and a capillary bridge is formed, exerting an attractive force on the particles. As the particles separate from each other again, the bridge stays intact up to a critical distance $d_{c}$. At this point the bridge ruptures [Willett et al., 2000]. Integrating this attractive force over the separation of the particles up to $d_{\mathrm{c}}$ yields the amount of energy which is dissipated in each bond rupture process.

This dissipative mechanism is an essential difference between wet and dry granular systems: The collisions of dry granulates are purely repulsive and characterized by the coefficient of restitution $\varepsilon$, which specifies which fraction of the kinetic energy is dissipated. Wet granular particles, however, are characterized by a hysteretic attractive interaction giving rise to a fixed amount of energy, which is dissipated in each rupture.

This existence of a well defined energy scale, which is absent in dry materials, is the essential microscopic ingredient not only of wet granulates but also of cohesive gases. In fact the liquid bridge can be thought of as a particular realization of a more general cohesive force. A particularly important aspect of free cooling in cohesive gases is the aggregation process which sets in, when the kinetic energy falls below the bond breaking energy. Wet granular systems may provide a realization of various aggregation models and so-called sticky gases [Carnevale et al., 1990], where particles move diffusively or ballistically until they collide and get stuck to an aggregate which is thereby growing. Such models have attracted a lot of interest [Liang \& Kadanoff, 1985; Jiang \& Leyvraz, 1993, 1994; Carnevale et al., 1990; Alves \& Ferreira, 2006; van Dongen \& Ernst, 1985; Westbrook et al., 2004; Jullien \& Kolb, 1984; Trizac \& Krapivsky, 2003; Trizac \& Hansen, 1995], due to a wide range of applications ranging from the formation of dust filaments, snowflakes and clouds to the size distribution and impact probability of planetesimals in accretion disks.

Kinetic properties of granular gases have been discussed mainly for dry materials. 
In particular, free cooling has been studied extensively [Ben-Naim et al., 1999; Nie et al., 2002; Baldassarri et al., 2002; Krapivsky \& Ben-Naim, 2002], and it was shown that the dissipative interactions are responsible for many novel phenomena, unexpected from the kinetic theory of molecular gases: The particles' velocities are not distributed according to a Maxwell-Boltzmann distribution [Goldshtein \& Shapiro, 1995], equipartition does not hold [Huthmann \& Zippelius, 1997; Garzo \& Dufty, 1999; Losert et al., 1999], a spatially homogeneous state is generically unstable [Goldhirsch \& Zanetti, 1993; Goldhirsch et al., 1993; McNamara, 1993], and linear and angular motion are correlated [Brilliantov et al., 2007].

Much less is known about wet granular media, which have been addressed only recently [Thornton et al., 1996; Lian et al., 1998; Huang et al., 2005; Herminghaus, 2005; Zaburdaev et al., 2006; Fingerle \& Herminghaus, 2006, 2008; Fingerle et al., 2008], focussing on nonequilibrium phase transitions [Fingerle et al., 2008], the equation of state [Fingerle \& Herminghaus, 2008], agglomeration [Ennis et al., 1991; Thornton et al., 1996; Lian et al., 1998], shear flow [Huang et al., 2005], and cooling in one dimension [Zaburdaev et al., 2006; Fingerle \& Herminghaus, 2006].

Structure formation in wet granulates during free cooling is the focus of this chapter, which is organized as follows: In Sec. 5.2 the model is introduced, and the decay of the average kinetic energy is discussed in Sec. 5.3. Aggregation is investigated in Sec. 5.4, before we conclude in Sec. 5.5. A short summary of the presented results has appeared in [Ulrich et al., 2009b] and an even shorter one in [Ulrich et al., 2009a].

\subsection{Models}

We are interested in the zero-gravity free cooling dynamics of wet granular gases. We assume the particles to be covered by a thin liquid film, as it is the case if the liquid completely wets the particle material [Israelachvili, 1992]. The particles move freely, until these surface films come into contact. The liquid then rapidly accumulates around the contact due to the interfacial forces. A capillary bridge forms at the contact, exerting an attractive force on the grains due to its negative Laplace pressure. This liquid bridge is stretched but stays intact (or even continues to grow) as the particles move apart. The attractive force thus remains until a certain critical separation $s_{\mathrm{c}}$ is reached, where the liquid neck becomes unstable and ruptures. As mentioned above, the hysteretic formation and rupture of the bridge gives rise to a characteristic loss of energy, $\Delta E$, which depends upon the thickness of the liquid film wetting the grains.

In order to design a suitable model, we briefly discuss the nature of this process. The formation of capillary bridges is quite fast in real systems. Between typical grains of one millimeter diameter it takes less than a millisecond [Herminghaus, 2005, Sec. II D]. It is clear, however, that this formation cannot in general be considered instantaneous if the velocity of the impacting grains $v_{\mathrm{i}}$ is large. If the time scale of the impact process, which may be written as $s_{\mathrm{c}} / v_{\mathrm{i}}$, is of the same order 
or even smaller than the time of capillary bridge formation, the accumulated liquid volume of the bridge, and hence $\Delta E$, will be smaller than for slow impacts. However, this will not greatly affect the main features of the wet system, in particular as to its characteristic difference from the dry granulate. In order to see that, we compare the effective restitution coefficient of the dry and of the wet system. This is shown in Fig. 5.1, where the restitution coefficient for the dry system is shown as the dotted curve. It tends to be mildly depending on impact energy [Brilliantov \& Pöschel, 2004], $E_{\mathrm{i}}$, with a negative slope throughout. The effective restitution coefficient of the wet system, $\varepsilon_{\text {eff }}=\sqrt{1-\Delta E / E_{\mathrm{i}}}$, is shown as the solid curve, assuming constant $\Delta E$. In strong contrast to the dry system, it has a zero at $\Delta E / E_{\mathrm{i}}=1$ and a markedly positive slope. This illustrates the dramatic difference between these two systems. The dashed line qualitatively accounts for the effect of finite formation time of the capillary bridge. Since $\varepsilon_{\text {eff }}$ must stay below one, the qualitative picture concerning the comparison of dry and wet granular gases remains unchanged.

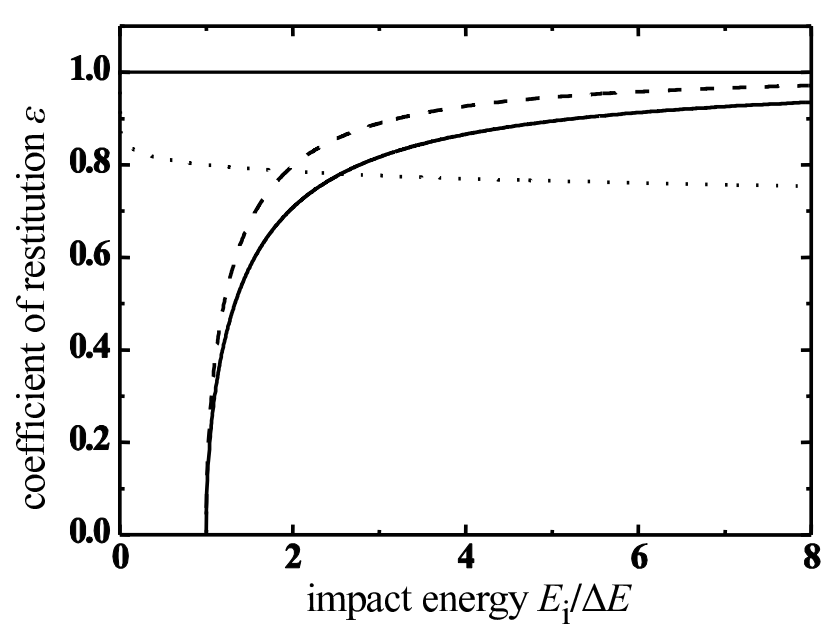

Figure 5.1: Restitution coefficients for dry (dotted) and wet (solid and dashed) granular systems, plotted vs. the impact energy in units of the wet energy loss, $\Delta E$. The main feature in the wet case is the zero at $E_{\mathrm{i}}=\Delta E$, which is unchanged if the finite formation time for capillary bridges is taken into account (dashed curve). The horizontal line $\varepsilon=1$ corresponds to the limit of fully elastic spheres.

Our system consists of $N$ identical and spherical particles with diameter $d$ and mass $m$ in a three-dimensional cubic volume $V=L^{3}$. They are at positions $\mathbf{r}_{1}, \ldots, \mathbf{r}_{N}$ and have the velocities $\mathbf{v}_{1}, \ldots, \mathbf{v}_{N}$. The particles have a hard-core interaction, such that two particles are reflected elastically, if their centers of mass reach the hard-core distance, which is the particle diameter $d$.

To account for the liquid film, a liquid bridge is allowed to form between a pair of particles if they come close enough ("close enough" is specified later). When these particles are moving apart and their distance exceeds the bond breaking distance $d_{\mathrm{c}}$, the liquid bridge will break and a fixed amount of kinetic energy $\Delta E$ is dissipated; in that process, momentum is conserved and the relative velocity $v_{\text {rel }}$ changes to $v_{\text {rel }}^{\prime}$ 


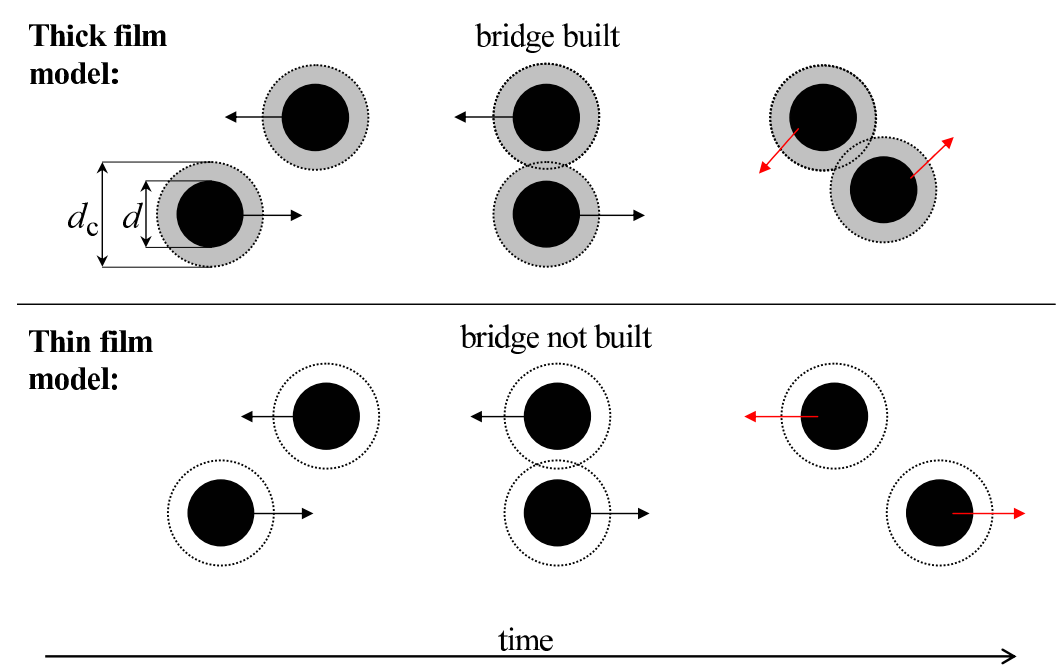

Figure 5.2: Illustration of the thin film model and thick film model. In the thick film model, the liquid bridge forms, as soon as the bond breaking distances $d_{\mathrm{c}}$ overlap. The same initial configuration in the thin film model does not create a liquid bridge, since the hard cores of the particles do not touch. Thus, the particles just pass by.

according to

$$
\frac{\mu}{2} v_{\text {rel }}^{\prime 2}=\frac{\mu}{2} v_{\text {rel }}^{2}-\Delta E
$$

with the reduced mass $\mu=m / 2$. If, however, the relative kinetic energy is smaller than $\Delta E$, the particles are elastically reflected towards each other. In this case the two particles oscillate between $d$ and $d_{\mathrm{c}}$, until a collision with a third particle or a wall supplies enough energy to break the liquid bridge.

The effect of the capillary force, which is present in reality for distances up to $d_{\mathrm{c}}$, is thus solely modeled by the energy loss which occurs at the distance $d_{\mathrm{c}}$. This has been shown before to be a very good approximation [Fingerle et al., 2008], and enables event-driven simulations as discussed below. For the formation of the liquid bridge, we distinguish between two models:

In the thick film model, a liquid bridge forms as soon as particles come closer than the critical bond breaking distance $d_{\mathrm{c}}$. This model assumes that the outer diameter of the liquid film is $d_{\mathrm{c}}$ and its shape stays spherical and is not deformed by the particles.

In the thin film model, the liquid bridge forms when the particles touch, i.e. the distance of their centers is equal to $d$. This model assumes that the liquid film covering the particles is infinitesimally thin and the capillary bridges form a thin liquid neck, which breaks off at the critical distance $d_{c}$. Raw data for this model was provided by courtesy of the Max Planck Institute for Dynamics and Self-Organization.

As it will turn out, the differences in most of the results are only minute. The two models are illustrated in Fig. 5.2. Note that in the limit $d_{\mathrm{c}} \rightarrow d$ the difference between the two models vanishes, since in a collision the bond breaking distance 
and hard core distance are always passed concurrently. For the sake of clarity, in all graphs throughout this chapter data points displayed as filled symbols (like •) represent the thick film model and open symbols (like o) the thin film model.

In general there is some energy being transferred to the atomic degrees of freedom of wet grains as well. We are going to neglect this dissipation mechanism for our simple model, because it is usually small as compared to the energy loss due to the breaking of capillary bridges, especially if the granular temperature is small. It is evident, however, that such a dissipation mechanism can easily be incorporated in the simulations, replacing the elastic reflection by incomplete normal restitution. We restrict ourselves here to perfectly smooth particles, such that translational and rotational motion are decoupled. Furthermore, we investigate free cooling only, so no gravity is present, and no energy is injected into the system.

The particular way of accounting for the liquid film used in these models makes it possible to use an event-driven simulation scheme. The possible events are the reflection of the particles at the hard core distance $d$ and the crossing of the bondbreaking distance $d_{\mathrm{c}}$, at which the bond-breaking energy $\Delta E$ is dissipated. Fingerle et al. [2008] have previously compared event-driven simulations of the wet system with full molecular dynamics simulations, integrating the equation of motion; they found good quantitative agreement in the results of both methods, justifying the event-driven approach chosen exclusively for the present study.

We use dimensionless units such that $\Delta E=1$, particle mass $m=1$ and particle diameter $d=4$. The bond-breaking distance is chosen as $d_{\mathrm{c}}=1.07 d$, unless noted otherwise, and volume fraction, $\phi=\pi d^{3} / 6 \cdot N / V$, is varied from $\phi \approx 0.06 \%$ up to $15.6 \%$. We use periodic boundary conditions in the $x$ - and $y$-direction and hard walls in $z$-direction. The hard walls are completely elastic and do not exert capillary interactions with the particles.

\subsection{Cooling Dynamics}

The central quantity in this section is the granular temperature $T$, defined via

$$
\frac{3}{2} T=\frac{1}{N} \sum_{i=1}^{N} \frac{m \mathbf{v}_{i}^{2}}{2} .
$$

We will investigate its decay in time from a given initial value $T_{0} \gg \Delta E$; in all the simulations $T_{0}=45 \Delta E$ was chosen.

Simple arguments can be used to derive an analytical form of the temperature decay. Therefore we first need the collision frequency $f_{\text {coll }}$, which is defined as the average number of collisions per time for a given particle. Since each collision involves two particles, $f_{\text {coll }} \cdot N \cdot \Delta t$ adds up to twice the number of collisions in the whole system during the short time interval $\Delta t$. In each collision a capillary bridge ruptures with probability $P_{\mathrm{bb}}$, giving rise to dissipation of a fixed amount of energy, the bond-breaking energy $\Delta E$. Hence, during this time the total kinetic 
energy decreases by

$$
\Delta E_{\mathrm{kin}}=\Delta E \cdot \frac{N f_{\mathrm{coll}} \Delta t}{2} \cdot P_{\mathrm{bb}} .
$$

Dividing by $N$ and $\Delta t$ yields, in the limit $\Delta t \rightarrow 0$, a rate equation for the temperature:

$$
\frac{3}{2} \frac{\mathrm{d} T}{\mathrm{~d} t}=-\frac{1}{2} \cdot f_{\mathrm{coll}} \cdot \Delta E \cdot P_{\mathrm{bb}}
$$

\subsubsection{Early Stage of Cooling}

In the early stage of cooling, the average kinetic energy per particle is much larger than the bond breaking energy, so that $P_{\mathrm{bb}} \approx 1$ and almost every collision gives rise to dissipation by $\Delta E$. For a dilute gas, the collision frequency

$$
f_{\text {coll }}=4 g(d) \sigma n \sqrt{\frac{T}{\pi m}}
$$

is well established [e.g. Brilliantov \& Pöschel, 2004], with the particle density $n=N / V$ and the pair correlation function at contact $g(d)$, for which we use the Carnahan-Starling approximation [Carnahan \& Starling, 1969], $g(d)=\frac{(2-\phi)}{2(1-\phi)^{3}}$. The two models differ only in the scattering cross section $\sigma$ (see Fig. 5.2), which is given by $\sigma=d^{2} \pi$ in the thin film model and $\sigma=d_{\mathrm{c}}^{2} \pi$ in the thick film model.

Applying Eq. (5.5) and $P_{\mathrm{bb}}=1$ to Eq. (5.4), gives rise to the following simple equation:

$$
\frac{\mathrm{d} T}{\mathrm{~d} t}=-\frac{4}{3} g(d) \sigma n \sqrt{\frac{T}{\pi m}} \Delta E \propto-\sqrt{T},
$$

which is solved by $T(t) \sim\left(t-t_{0}\right)^{2}$. Inserting the prefactors and setting the initial value $T(0)=T_{0}$, we obtain, similar to Haff's law [Haff, 1983], an analytical form of the decay of the temperature:

$$
T(t)= \begin{cases}T_{0}\left(1-t / t_{0}\right)^{2} & \text { for } t \leq t_{0} \\ 0 & \text { for } t>t_{0}\end{cases}
$$

with a characteristic time scale

$$
t_{0}=\frac{3 \sqrt{\pi m T_{0}}}{2 g(d) \sigma n \Delta E}
$$

Note that, in this simplified model, the assumption that every collision causes an energy loss $\Delta E$ gives rise to a time-scale $t_{0}$ after which all energy is dissipated. Even though this assumption does not hold for all times in the simulation (since the bonds do not break anymore if the relative kinetic energy is too small), the timescale 
$t_{0}$ has a clear physical relevance. It sets the time after which the temperature is comparable to the bond-breaking energy $\Delta E$ and after which persistent clusters will form.

Now, let us have a look at the evolution of the granular temperature $T$ from the simulation and compare it to (5.7): In Fig. 5.3 the temperature of the thick film model is shown for different volume fractions. One can see that the agreement between theory and simulation is quite good up to $t_{0}$, when the assumption contained in Eq. (5.4) that every collision causes an energy loss $\Delta E$ breaks down.

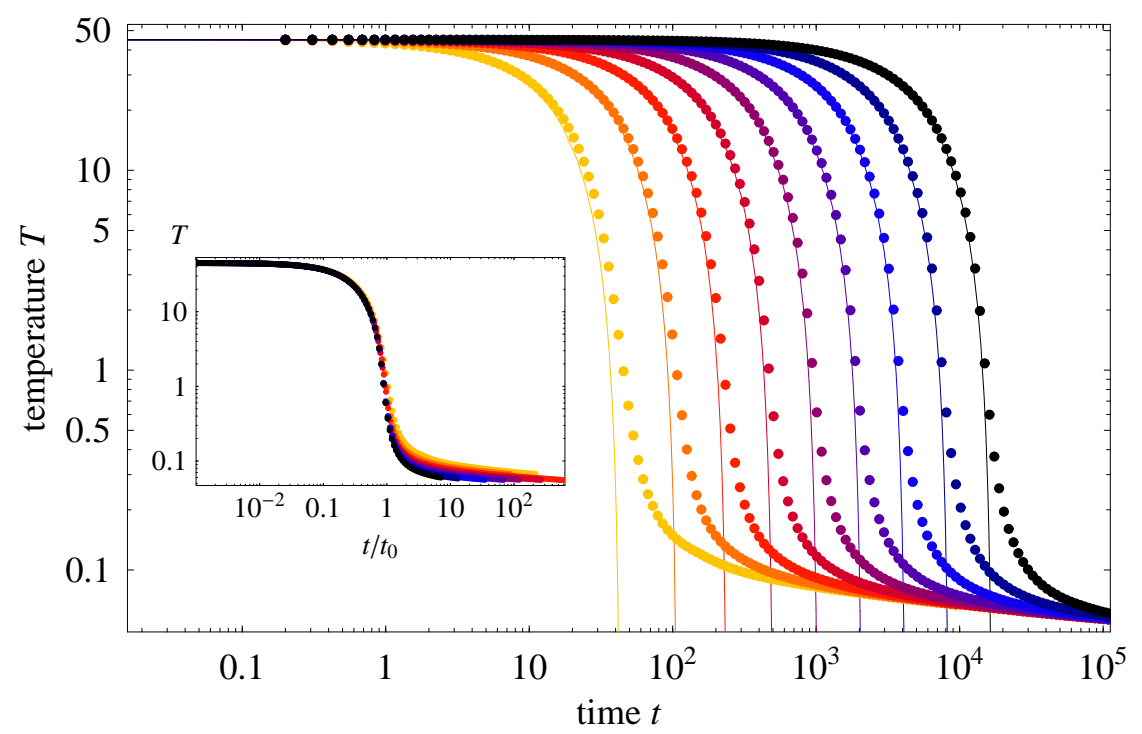

Figure 5.3: Decay of the granular temperature $T$ for volume fractions (from left to right) $\phi=15.6 \%, 7.81 \%, 3.90 \%, 1.95 \%, 0.98 \%, 0.49 \%, 0.24 \%, 0.12 \%, 0.061 \%$; the number of particles $N=262144$ is fixed; comparison of theory (full line) and simulation $(\bullet)$. Inset: $T$ versus scaled time $t / t_{0}$.

In the simplified cooling law (5.7a), the volume fraction only enters into $t_{0}$. Hence we can try to superimpose the data by scaling time with $t_{0}$. As can be seen in the inset of Fig. 5.3, the data obey the expected scaling well, except for the long time limit, which has different asymptotic behavior and is treated in the next section, Sec. 5.3.2.

In Fig. 5.4, the temperature evolution of the thick and thin film model is compared. The difference of the two models is solely due to different scattering crosssections, entering in $t_{0}$, Eq. (5.7b). Again, we observe good agreement of the simplified theory and the simulation up to the time $t_{0}$. In the inset the time is rescaled with $t_{0}$. 


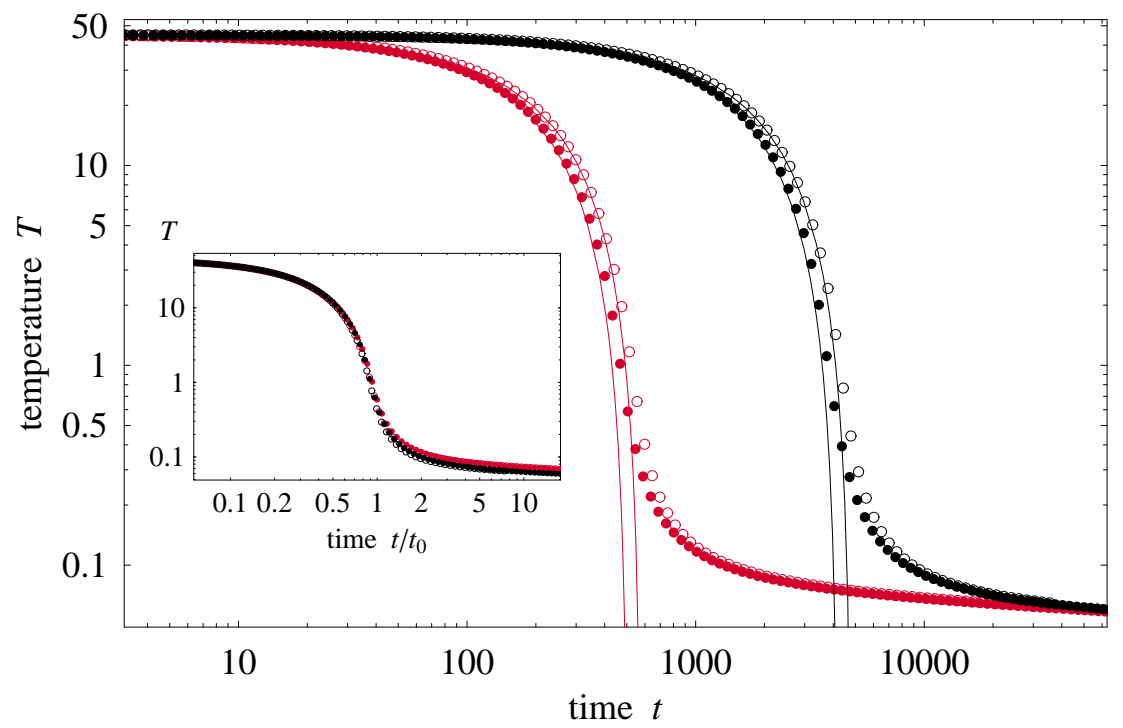

Figure 5.4: Decay of the granular temperature $T$ for the thick film model $(\bullet)$ and the thin film model (०) and for volume fractions $\phi=1.95 \%$ (red) and $\phi=0.24 \%$ (black). The inset shows $T$ versus scaled time $t / t_{0}$.

In Fig. 5.5, we investigate how the the bond breaking distance $d_{\mathrm{c}}$ influences the cooling behavior. As mentioned above, this will influence the scattering cross section $\sigma=d_{\mathrm{c}}^{2} \pi$ for the thick film model and according to Eq. (5.7b), and $t_{0}$ should increase when decreasing $d_{\mathrm{c}}$. This behavior can indeed be observed on the left side of Fig. 5.5, which shows the data for the thick film model.
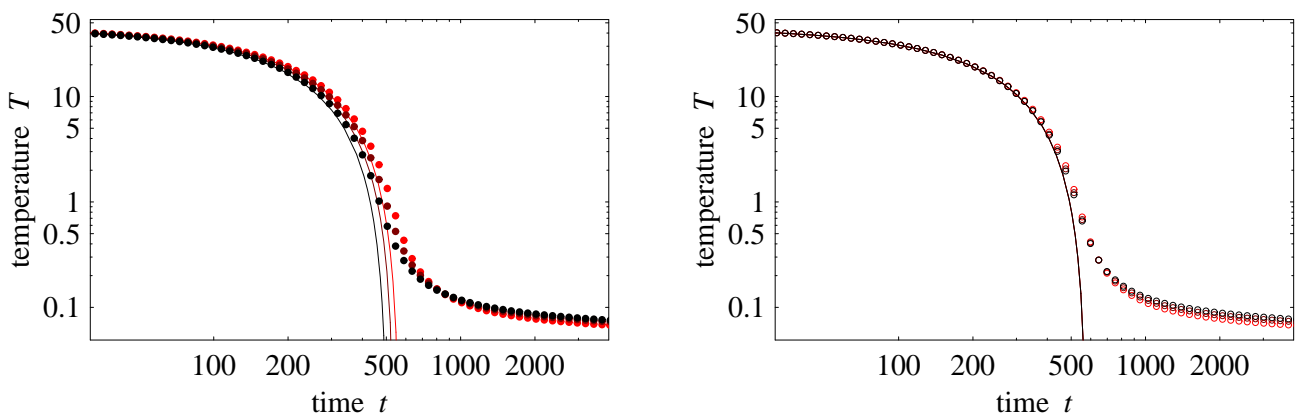

Figure 5.5: Decay of the granular temperature $T$ for volume fraction $\phi=1.95 \%$ and $N=262144$ particles. The bond breaking distances are $d_{\mathrm{c}}=1.07 d$ (black), $d_{\mathrm{c}}=1.035 d$ (brown) and $d_{\mathrm{c}}=1.01 d$ (red). The left graph shows data for the thick film model, the right graph for the thin film model.

For the thin film model, the scattering cross section $\sigma=d^{2} \pi$ depends only on the hard core diameter $d$ of the particles. Hence varying $d_{\mathrm{c}}$ should not influence the time $t_{0}$. This can be seen on the right hand side of Fig. 5.5, where the different curves of 
the thin film model coincide well, until the time $t_{0}$ is passed and aggregation starts.

Surprisingly one can see that in the aggregated state $t>t_{0}$ a smaller bond breaking distance $d_{\mathrm{c}}$ slightly expedites the cooling process, for both models. This is probably because a smaller $d_{\mathrm{c}}$ increases the collision frequency of the particles inside clusters.

\subsubsection{Late Stage of Cooling}

In the late stage of aggregation, when the system is strongly aggregated, it becomes very unlikely that a capillary bridge ruptures and hence we observe a very slow time evolution of our system. The slow decrease of the temperature can be understood with simple arguments. Therefore we start with Eq. (5.4),

$$
\frac{3}{2} \frac{\mathrm{d} T}{\mathrm{~d} t}=-\frac{1}{2} f_{\text {coll }} \Delta E P_{\mathrm{bb}},
$$

as in the previous section, but omit the assumption $P_{\mathrm{bb}} \approx 1$. The probability $P_{\mathrm{bb}}$ to break a bond is given by the probability to find a kinetic energy larger than $\Delta E$ :

$$
P_{\mathrm{bb}}=\int \mathrm{d} \mathbf{v} \theta\left(m \mathbf{v}^{2} / 2-\Delta E\right) w(\mathbf{v}),
$$

where $w(\mathbf{v})$ is the velocity distribution. As we will see in Sec. 5.3.3, in the clustered state it is reasonable to approximate it by a Maxwellian

$$
w(\mathbf{v})=\left(\frac{m}{2 \pi T(t)}\right)^{3 / 2} \exp \left(-\frac{m \mathbf{v}^{2}}{2 T(t)}\right) .
$$

As it is usually done for spherical symmetrical integrals like (5.9), we can integrate out the angular variables, yielding a factor of $4 \pi v^{2}$, and then consider the dependence of the absolute value $v=|\mathbf{v}|$ :

$$
\begin{aligned}
P_{\mathrm{bb}} & =4 \pi\left(\frac{m}{2 \pi T(t)}\right)^{3 / 2} \int_{\sqrt{2 \Delta E / m}}^{\infty} \mathrm{d} v v^{2} \exp \left(-\frac{m v^{2}}{2 T(t)}\right) \\
& =\sqrt{\frac{4 \Delta E}{\pi T}} \exp \left(-\frac{\Delta E}{T}\right)+\operatorname{erfc}\left(\sqrt{\frac{\Delta E}{T}}\right) .
\end{aligned}
$$

We are interested in the asymptotic limit $T(t) / \Delta E \rightarrow 0$. Looking up the asymptotic expansion of the complementary error function [e.g. Stegun \& Abramowitz, 1954, Ch. 7],

$$
\operatorname{erfc}(x)=\frac{\exp \left(-x^{2}\right)}{x \sqrt{\pi}}\left(1+\mathcal{O}\left(x^{-2}\right)\right) \quad \text { for } \quad x \rightarrow \infty,
$$

we realize that the contribution of the $\operatorname{erfc}(. .$.$) -term only makes a lower order con-$ tribution in the limit $T / \Delta E \rightarrow 0$ and hence:

$$
P_{\mathrm{bb}}=\sqrt{\frac{4 \Delta E}{\pi T}} \exp \left(-\frac{\Delta E}{T}\right) \cdot\left(1+\mathcal{O}\left(\frac{T}{\Delta E}\right)\right) .
$$


The collision frequency $f_{\text {coll }}$ is not known for the clustered state, but it should be proportional to $T^{1 / 2}$ : Increasing all particle velocities for a given system by a factor of two must also increase the collision frequency by a factor of two:

$$
f_{\text {coll }}=\gamma T^{1 / 2} .
$$

Roughly speaking, the prefactor $\gamma$ contains information about how large the local density around a given particle is, i.e. how many close-by particles a given particles has to collide with. Hence, $\gamma$ will clearly be higher in the clustered state than in the homogeneous state. By assuming that $\gamma$ is not time dependent itself, we assume that the internal structure within the clusters does not change significantly as $t \rightarrow \infty$ (while the cluster is still allowed to change its overall shape). This seems to be a good assumption in the highly clustered state, however, it certainly can not correctly describe the transition state, where particles conglomerate around a given particle and the local density changes drastically ${ }^{1}$.

With that, the rate equation (5.8) becomes:

$$
\begin{aligned}
\frac{\mathrm{d} T}{\mathrm{~d} t} & =-\frac{\Delta E}{3} \gamma T^{1 / 2} \sqrt{\frac{4 \Delta E}{\pi T}} \exp \left(-\frac{\Delta E}{T}\right) \cdot\left(1+\mathcal{O}\left(\frac{T}{\Delta E}\right)\right) \\
\frac{1}{\Delta E} \frac{\mathrm{d} T}{\mathrm{~d} t} & =-\nu \exp \left(-\frac{\Delta E}{T}\right) \cdot\left(1+\mathcal{O}\left(\frac{T}{\Delta E}\right)\right),
\end{aligned}
$$

where we defined $\nu=\frac{2}{3 \sqrt{\pi}} \cdot \gamma \Delta E^{1 / 2}$, which has the dimension of a frequency ${ }^{2}$. To solve this equation, it is useful to introduce the dimensionless inverse temperature:

$$
x:=\frac{\Delta E}{T} .
$$

With that, Eq. (5.15) becomes:

$$
\begin{aligned}
\frac{\mathrm{d}\left(x^{-1}\right)}{\mathrm{d} t}= & -\frac{1}{x^{2}} \frac{\mathrm{d} x}{\mathrm{~d} t}=-\nu \mathrm{e}^{-x} \cdot\left(1+\mathcal{O}\left(x^{-1}\right)\right) \\
& \Rightarrow \quad \frac{\mathrm{d} x}{\mathrm{~d} t}=\nu x^{2} \mathrm{e}^{-x} \cdot\left(1+\mathcal{O}\left(x^{-1}\right)\right) .
\end{aligned}
$$

This differential equation can be solved by separation of variables:

$$
\int \mathrm{d} x x^{-2} \mathrm{e}^{x} \cdot\left(1+\mathcal{O}\left(x^{-1}\right)\right)=\nu \int \mathrm{d} t .
$$

The integration of the right hand side is trivial. For the left hand side we find by repeated partial integration that:

$$
\begin{array}{r}
\int \mathrm{d} x x^{-2} \mathrm{e}^{x}=x^{-2} \mathrm{e}^{x}+\mathcal{O}\left(x^{-3} \mathrm{e}^{x}\right), \\
\text { and } \int \mathrm{d} x x^{-3} \mathrm{e}^{x}=x^{-3} \mathrm{e}^{x}+\mathcal{O}\left(x^{-4} \mathrm{e}^{x}\right) .
\end{array}
$$

\footnotetext{
${ }^{1}$ Indeed, attempts to describe the temperature evolution in the transition state with this formalism failed.

${ }^{2}$ comparing it to Eq. (5.14) indicates, it is roughly the collision frequency, when $T=\Delta E$.
} 
Hence Eq. (5.18) becomes:

$$
x^{-2} \mathrm{e}^{x} \cdot\left(1+\mathcal{O}\left(x^{-1}\right)\right)=\nu t+c,
$$

where $c$ is an integration constant, which has to be chosen to match the initial condition. Figuratively speaking, we can use $c$ to shift the solution $x(t)$ along the time axis back and forth and thereby run through the set of solutions of the differential equation (5.17). However, we have to keep in mind that this equation is derived in the limit $x=\Delta E / T \gg 1$; therefore this initial condition has to be set in the low temperature regime, as well, and cannot be chosen as $T(0)=T_{0} \gg \Delta E$ as in the previous section.

In order to see how the temperature evolves asymptotically in the limit $t \rightarrow \infty$, we need to invert Eq. (5.21), which is done in Appendix D.2:

$$
x(t)=\ln \tilde{t}+2 \ln \ln \tilde{t}+\frac{4 \ln \ln \tilde{t}}{\ln \tilde{t}}+\mathcal{O}\left(\frac{1}{\ln \tilde{t}}\right),
$$

with $\tilde{t}=\nu t+c$. If we turn back to the actual temperature and resubstitute $T(t) / \Delta E=x^{-1}(t)$, we get in highest possible order from Eq. (5.22):

$$
\frac{T(t)}{\Delta E}=\frac{1}{\ln \tilde{t}+2 \ln \ln \tilde{t}+\frac{4 \ln \ln \tilde{t}}{\ln \tilde{t}}}+\mathcal{O}\left(\frac{1}{(\ln \tilde{t})^{3}}\right)
$$

and lower orders

$$
\begin{aligned}
\frac{T(t)}{\Delta E} & =\frac{1}{\ln \tilde{t}+2 \ln \ln \tilde{t}}+\mathcal{O}\left(\frac{\ln \ln \tilde{t}}{(\ln \tilde{t})^{3}}\right) \\
& =\frac{1}{\ln \tilde{t}}+\mathcal{O}\left(\frac{\ln \ln \tilde{t}}{(\ln \tilde{t})^{2}}\right) .
\end{aligned}
$$

If furthermore $\nu t \gg c$ we can also neglect $c$. This is because the terms $\ln (\nu t+c)$ and $\ln \ln (\nu t+c)$ produce corrections of at least $\mathcal{O}\left(\frac{c}{\nu t}\right)$, which one can easily confirm. Hence we find for the leading order in $t$ :

$$
\frac{T(t)}{\Delta E}=\frac{1}{\ln \nu t}+\mathcal{O}\left(\frac{\ln \ln \nu t}{\ln \nu t}\right) .
$$

This result shows that the temperature exhibits a very slow, logarithmic time decay for $t \rightarrow \infty$, induced by the very low probability to break a bond, Eq. (5.13); this is in strong contrast to the algebraic time decay observed for granular systems with coefficient of restitution $\varepsilon<1$ [Brilliantov \& Pöschel, 2004].

The different orders of the approximations (5.23) as well as the numeric solution of the original differential equation (5.8) are shown in Fig. 5.6 and compared to the simulation data. The unknown prefactor $\gamma$, or equivalently $\nu$, is a fit parameter. As one can see, in the limit $t \rightarrow \infty$, all curves show the same asymptotic behavior, as expected. Approaching the transition time $t \rightarrow t_{0}$, the lowest order approximation (5.23d) clearly deviates first, while already the second lowest order shows (5.23b) roughly the same conformance as the higher orders. 


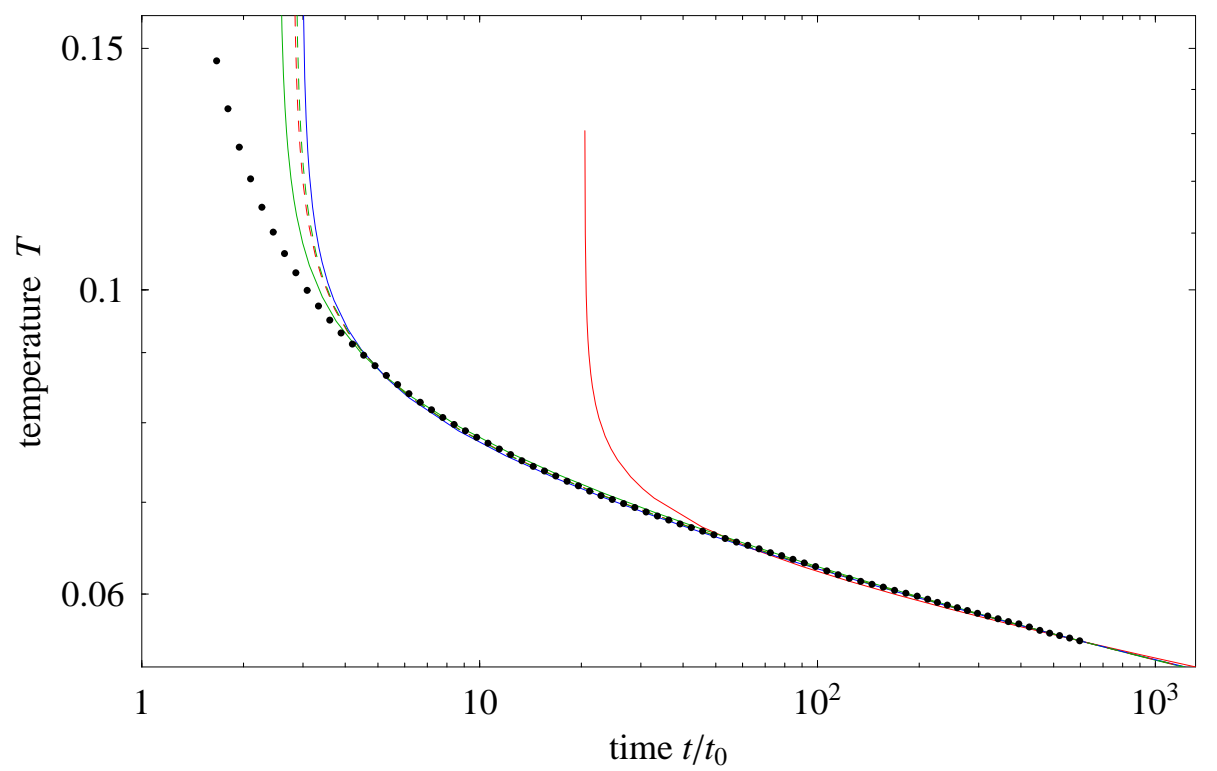

Figure 5.6: Asymptotic temperature evolution for a system with $\phi=3.9 \%$ and $N=262144$. The data points show the simulation data, while the lines show analytical forms with different orders of precision: The red line is the lowest order approximation $(5.23 \mathrm{c})$, the green line the second lowest $(5.23 \mathrm{~b})$ and the blue line the third lowest (5.23a). The dashed lines are numerical solutions of Eq. (5.8) with $P_{\mathrm{bb}}$ given by Eq. (5.11) (red) and its first order approximation Eq. (5.13) (green).

In Fig. 5.7, the second order solution (5.23b) is compared to the simulation data for different volume fractions, showing good agreement. One can see that for all densities, the temperature approaches a universal curve as $t \rightarrow \infty$. Considering the logarithmic decay Eq. (5.23c), this is not surprising: Even if two systems have different values for $\nu$, let's say $\nu_{1}$ and $\nu_{2}$, then in the limit $t \rightarrow \infty$

$$
\begin{aligned}
\frac{T_{\nu_{2}}(t)}{\Delta E} & =\frac{1}{\ln \nu_{2} t}=\frac{1}{\ln \nu_{1} t+\ln \nu_{2} / \nu_{1}}=\frac{1}{\ln \nu_{1} t}+\mathcal{O}\left(\left(\ln \nu_{1} t\right)^{-2}\right) \\
& =\frac{T_{\nu_{1}}(t)}{\Delta E}+\mathcal{O}\left(\left(\ln \nu_{1} t\right)^{-2}\right)
\end{aligned}
$$

Hence, the difference between the curves vanishes. 


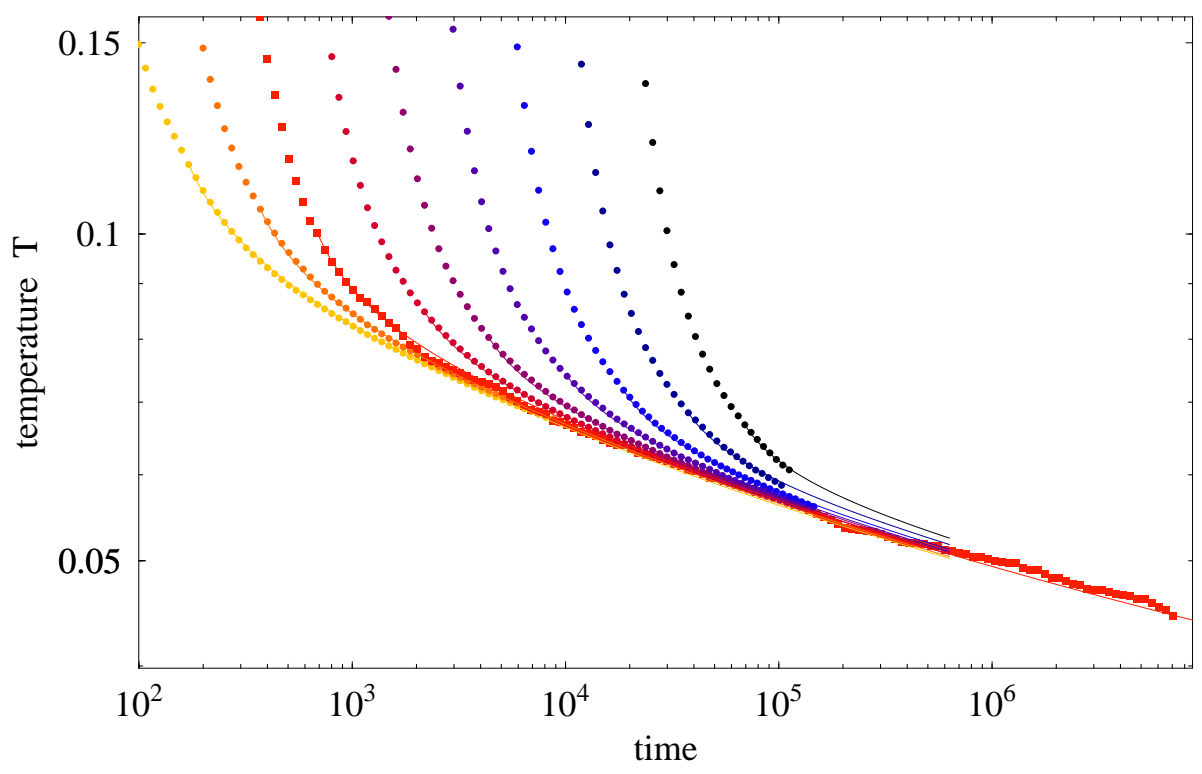

Figure 5.7: Asymptotic time dependence of the temperature for volume fractions (from left to right) $\phi=15.6 \%, 7.81 \%, 3.90 \%, 1.95 \%, 0.98 \%, 0.49 \%, 0.24 \%, 0.12 \%$, $0.061 \%$. The number of particles is $N=262144$, except for the third curve ( $\bullet$ ), which has $N=8192$ particles and is therefore somewhat noisy, but shows about two more decades in time; data (dots) in comparison to the analytical results, Eq. (5.23b) (lines) 


\subsubsection{Velocity Distribution}

In this section we have a look at the velocity distribution $w(\mathbf{v})$, which is the probability for a particle to have the velocity $\mathbf{v}$. Fundamental for molecular gases is the Maxwell distribution; however, for dissipative systems, it is well known that deviations from the Maxwellian occur. This is found in experimental work as well as simulations and for free cooling as well as driven systems. However, the actual shape of the velocity distribution is still under discussion. In most studies the highly energetic particles are overpopulated, resulting in so called "high energy tails".

Esipov \& Pöschel [1997]; Brey et al. [1999]; Huthmann et al. [2000] find that the velocity distribution for undriven dry systems decays exponentially $w(\mathbf{v}) \propto$ $\exp (-A v)$ in the high velocity limit. For a uniformly heated system, van Noije \& Ernst [1998] find that a stretched exponential $w(\mathbf{v}) \propto \exp \left(-A v^{3 / 2}\right)$ is a solution to the homogeneous Enskog-Boltzmann equation in arbitrary dimension in the high velocity limit. Ben-Naim \& Machta [2005]; Ben-Naim et al. [2005] additionally find stationary states with algebraic tails $w(\mathbf{v}) \propto v^{-\sigma}$, with a homogeneous model using a very general collision kernel. In a two-dimensional system, Goldhirsch et al. [1993] find that the velocity distribution deviates from the classical Maxwell-Boltzmann distribution as the clustering instability ${ }^{3}$ sets in.

Zaburdaev et al. [2006] studied a one-dimensional wet granular system (meaning a model with well defined bond breaking energy) in the limit of vanishing bond breaking distance $d_{\mathrm{c}} \rightarrow d$. It seems that the velocity distribution, initially set as Maxwellian, evolves towards an exponential profile, once the particles start to cluster. In that study, however, if the kinetic energy of a pair of particles is not sufficient to break a bond, all relative kinetic energy is dissipated and their relative motion is not considered anymore. Thus, clusters have no internal degrees of freedom and therefore, for late times, different results should be expected in our system.

In this regard, the question arises, how for the presented system the fixed bondbreaking energy and the clustering of the particles, starting at the transition time $t_{0}$, influences the velocity distribution. Fig. 5.8 shows the typical behavior of the velocity distribution. Given the anisotropic boundary conditions, we compare the velocity distributions in the direction of the periodic boundary conditions, $w\left(v_{x}\right)=$ $\int \mathrm{d} v_{y} \mathrm{~d} v_{z} w(\mathbf{v})$ (left), and the hard walls, $w\left(v_{z}\right)=\int \mathrm{d} v_{x} \mathrm{~d} v_{y} w(\mathbf{v})$ (right).

In the early stage of cooling, when $t \ll t_{0}$, the velocity distribution is well described by a Maxwellian. At the shown time in the top panel $t \approx 0.01 t_{0}$, each particle has had on average three collisions. In the transition regime, where $t \approx t_{0}$ and $T \approx \Delta E$, deviations from the Maxwellian distribution occur, characterized by a pronounced peak in the center and by high energy tails, as in most other dissipative systems. Interestingly, in the clustering regime $t \gg t_{0}$, the velocity distribution returns to a Maxwellian, again. Note also that $w\left(v_{x}\right)$ and $w\left(v_{z}\right)$ coincide during the investigated simulation time, which means that $w(\mathbf{v})$ remains isotropic.

\footnotetext{
${ }^{3}$ This so called clustering instability is excessively studied and produces spatial inhomogeneities of typical size $L_{0} \propto\left(1-\varepsilon^{2}\right)^{1 / 2}$, given that the system size is large enough $\left(L>L_{0}\right)$ and the system is given enough time to evolve.
} 

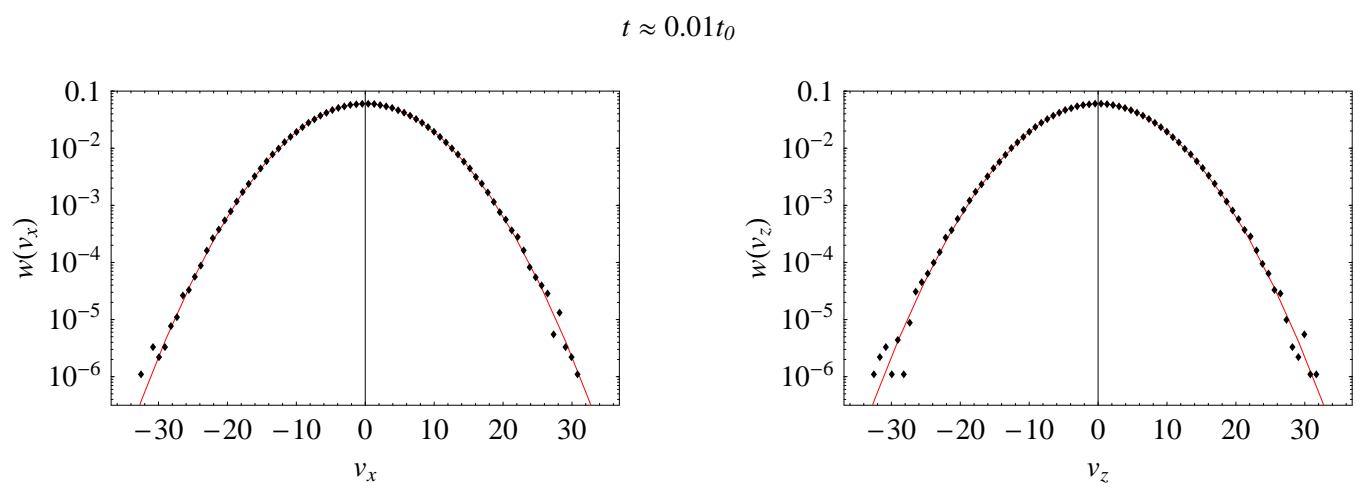

$t \approx t_{0}$
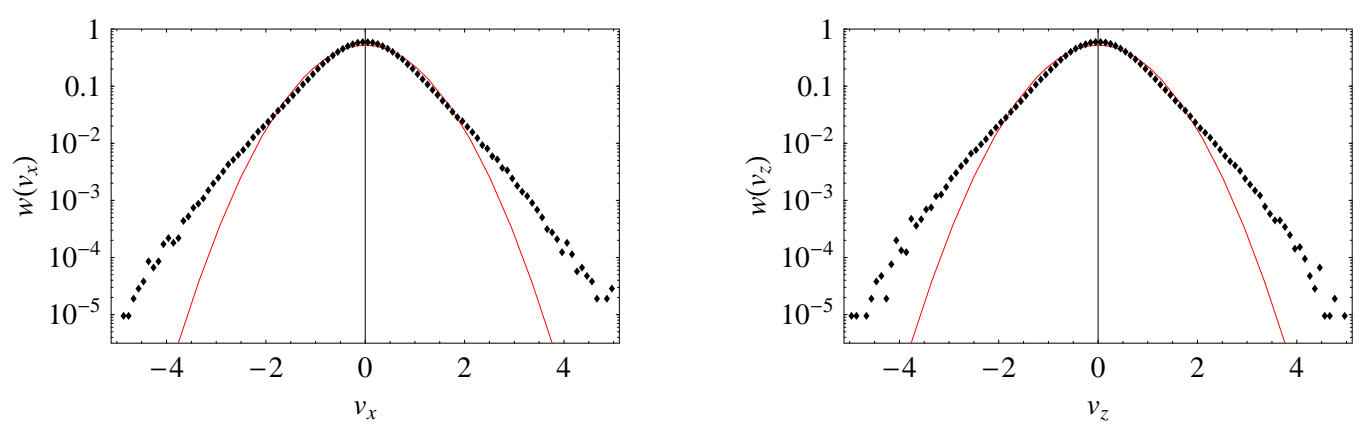

$t \approx 40 t_{0}$
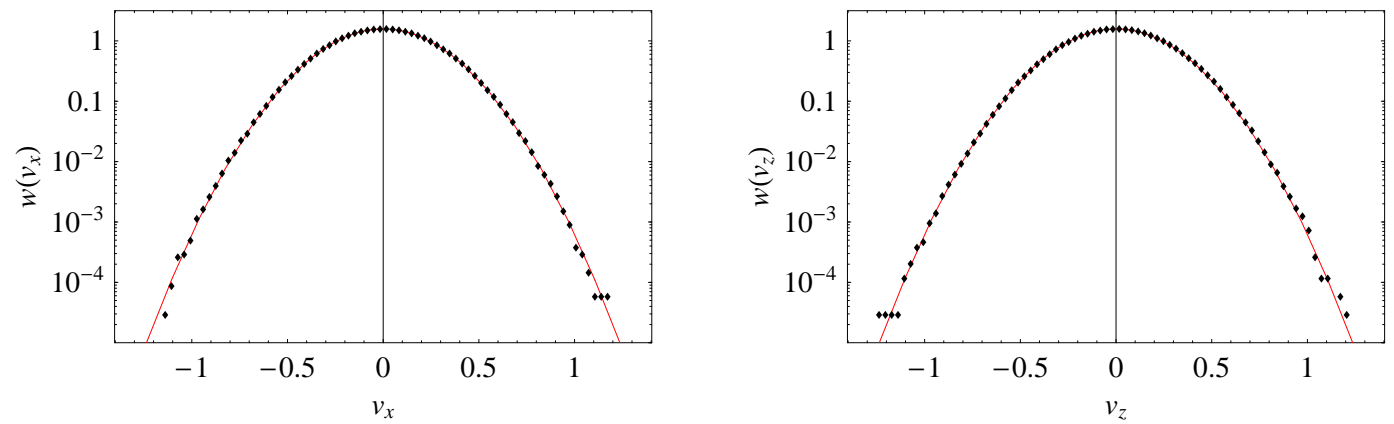

Figure 5.8: Velocity distributions $w\left(v_{x}\right)$ and $w\left(v_{z}\right)$ for times $t \approx 0.01 t_{0}$ in the free cooling regime (top), $t \approx t_{0}$ in the transition state (center) and $t \approx 40 t_{0}$ in the highly clustered state (bottom). The corresponding temperatures are $T / \Delta E=44$ (top), 0.59 (center) and 0.063 (bottom). The data points show the distribution for a system with $N=1048576$ particles and $\phi=1.96 \%$ and the solid lines are Maxwell distributions for the corresponding temperature at the given system time. 
In order to characterize the deviations from a Gaussian profile, one typically uses the kurtosis, defined as the ratio of the fourth moment and the variance squared [see Balanda \& MacGillivray, 1988; Chissom, 1970]:

$$
\beta_{2}=\frac{\left\langle v^{4}\right\rangle}{\left\langle v^{2}\right\rangle^{2}} .
$$

For a Gaussian distribution, one obtains $\beta_{2}=3$; thus deviations from 3 quantify deviations from the Gaussian distribution, in particular $\beta_{2}>3$ typically corresponds to over-populated tails and peaked centers (so called leptokurtic distributions) and $\beta_{2}<3$ vice versa (platykurtic distributions). For an exponential profile $\mathrm{e}^{-|v| / v_{0}}$, the kurtosis is $\beta_{2}=6$.

In our three dimensional case, every spatial dimension yields a separate value for the kurtosis. To see to what extent the anisotropy of the system plays a role, we keep the three separate values:

$$
\beta_{2, \nu}=\frac{\left\langle v_{\nu}^{4}\right\rangle}{\left\langle v_{\nu}^{2}\right\rangle^{2}} \quad \text { for } \quad \nu=x, y, z
$$

The time evolution of the kurtosis for $x$ - and $z$-dimension for the system in Fig. 5.8 is shown in Fig. 5.9. One observes a sharp peak with $\beta_{2}>3$ around the time $t_{0}$, when clustering sets is, while the rest of the evolution has the Gaussian-like value of $\beta_{2} \approx 3$. The differences between $x$ - and $z$-direction are marginal.

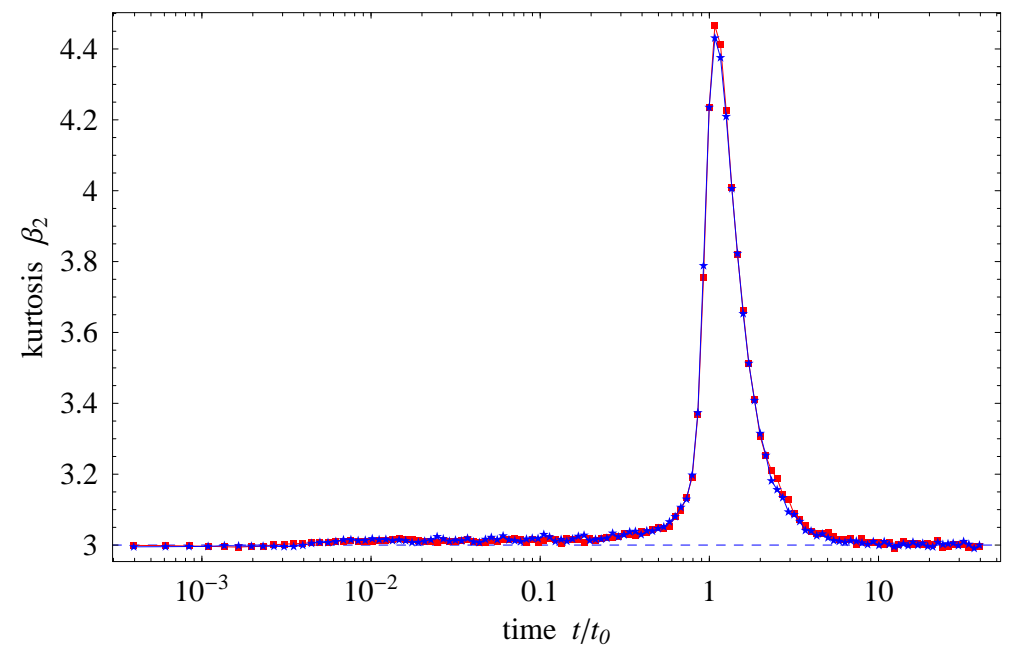

Figure 5.9: Time evolution of the kurtosis $\beta_{2}$ for the system of Fig. 5.8. Red boxes (匹) show $\beta_{2, x}$, blue stars $(\star)$ show $\beta_{2, z}$.

The intriguing fact that the velocity distribution returns to a Maxwell distribution can be understood with the help of results of the previous section 5.3.2, where we found that bond breaking events become extremely rare in the long time limit, $P_{\mathrm{bb}} \propto \exp (-\Delta E / T) ;$ consequently most collisions are elastic and the system can equilibrate. 
One might consider the behavior of the particles in a cluster, which reside in "cages" determined by the gap between the hard core distance and bond breaking distance, reminiscent of glassy dynamics. Indeed, a similar model of so called "sticky hard spheres" [Baxter, 1968], where hard spheres additionally have a very short ranged attractive (but not hysteretic) square well potential, is commonly used to study glassy structures. Unlike a glass, however, it is clear that in the presented system, the rearrangements of particles do cost energy, because breakage of bonds is hysteretic; thus the long time dynamics is expected to differ from glassy systems.

Fig. 5.10 compares the time evolution of the kurtosis of different volume fractions $\phi$. One can see that the value of the kurtosis around $t=t_{0}$ increases when going to more and more dilute systems. Since a kurtosis value of 3 is no guarantee for a Gaussian distribution, it was checked that every system indeed approaches a Maxwellian for large times and this is fulfilled very well.

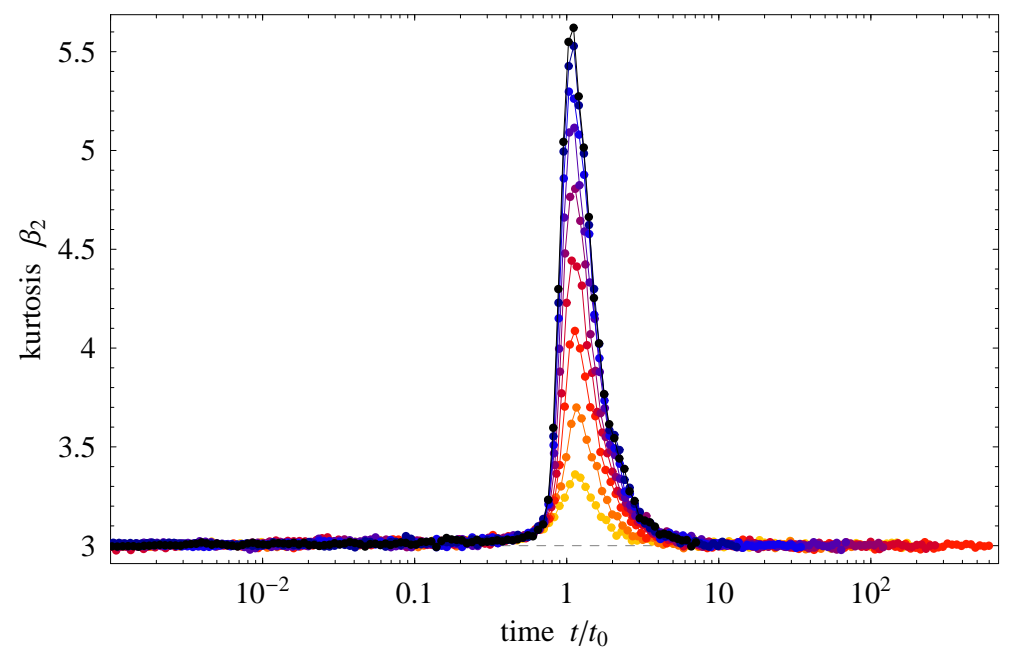

Figure 5.10: Time evolution of the kurtosis $\beta_{2, x}$ in $x$-direction for systems with $N=262144$ particles and volume fractions (from bottom to top) $\phi=15.6 \%$, $7.81 \%, 3.90 \%, 1.95 \%, 0.98 \%, 0.49 \%, 0.24 \%, 0.12 \%, 0.061 \%$. (same color coding as in Fig. 5.3)

\subsubsection{Partitioning of the Energy into Translational, Rotational and Internal Degrees of Freedom}

After the time $t_{0}$ has passed, stable clusters emerge. For the definition of a cluster, we define particles as neighbors, if a bridge is formed and the relative kinetic energy is not sufficient to break it. This makes sure that particles which are just "passing by", are not considered neighbors. A cluster is a set of particles connected through this neighbor-relationship. We refer to the number of particles a cluster contains as the cluster mass $m$. One should keep in mind that clusters defined in this way are not truly stable. Particles belonging to the cluster are occasionally kicked out, if hit by a very energetic particle. 
For a more detailed understanding of the system, we investigate the cooling dynamics on the cluster level, and determine how energy is partitioned among the degrees of freedom. We split the total temperature $T$ into three constituents, the translational temperature defined via the center-of-mass velocities of the clusters, the rotational temperature defined via the angular momenta of the clusters, and the internal temperature describing the relative movement of the particles inside a cluster. These three temperatures are defined as follows.

Our definition of neighborhood relations gives rise to $n_{\mathrm{cl}}$ distinct clusters numbered by $i=1, \ldots, n_{\mathrm{cl}}$. We denote by $\mathcal{N}_{i}$ the $i$-th cluster with $m_{i}$ particles. Its center of mass position and velocity are given by:

$$
\mathbf{R}_{i}=\frac{1}{m_{i}} \sum_{\nu \in \mathcal{N}_{i}} \mathbf{r}_{\nu} \quad \text { and } \quad \mathbf{V}_{i}=\frac{1}{m_{i}} \sum_{\nu \in \mathcal{N}_{i}} \mathbf{v}_{\nu}
$$

Note that single particles with $m_{i}=1$ are also considered as clusters.

The center of mass movement of each cluster has $f_{\text {trans }, i}=3$ translational degrees of freedom, so that the total number of translational degrees of freedom of these clusters is simply $3 n_{\mathrm{cl}}$. Homogeneous cluster translations are thus characterized by the translational temperature

$$
T_{\text {trans }}:=\frac{2}{3 n_{\mathrm{cl}}} \sum_{i=1}^{n_{\mathrm{cl}}} \frac{m_{i}}{2} \mathbf{V}_{i}^{2} .
$$

Analogously, the rotational temperature describes the energy in homogeneous cluster rotations. The angular momentum, $\mathbf{L}_{i}$, of cluster $i$ is given in terms of the relative particle positions $\tilde{\mathbf{r}}_{i, \nu}=\mathbf{r}_{\nu}-\mathbf{R}_{i}$ and velocities $\tilde{\mathbf{v}}_{i, \nu}=\mathbf{v}_{\nu}-\mathbf{V}_{i}$

$$
\mathbf{L}_{i}=\sum_{\nu \in \mathcal{N}_{i}} \tilde{\mathbf{r}}_{i, \nu} \times \tilde{\mathbf{v}}_{i, \nu}
$$

The rotational energy of cluster $\mathcal{N}_{i}$ with $m_{i}>2$ is thus given by

$$
E_{\mathrm{rot}, i}=\frac{1}{2} \mathbf{L}_{i} \underline{\underline{\mathrm{I}}}_{i}^{-1} \mathbf{L}_{i},
$$

where the moment of inertia tensor $\underline{\underline{I}}_{i}$ is defined in the usual way ${ }^{4}$. The case $m_{i}=2$, requires special treatment, since the inertia tensor is singular. The rotational energy of a dimer can be easily calculated to $E_{\mathrm{rot}, i}=\Delta \mathbf{v}_{i \perp}^{2} / 4$, where $\Delta \mathbf{v}_{i \perp}$ denotes the relative velocity perpendicular to the axis of the dimer. The rotational temperature is thus

$$
T_{\mathrm{rot}}:=\frac{2}{\sum_{i=1}^{n_{\mathrm{cl}} f_{\mathrm{rot}, i}}} \sum_{i=1}^{n_{\mathrm{cl}}} E_{\mathrm{rot}, i},
$$

with $f_{\text {rot }, i}=2$ for dimers and $f_{\text {rot }, i}=3$ for larger clusters.

All the left-over kinetic energy $E_{\text {int }}$ describes the relative movement of particles inside a cluster and contributes to the internal temperature. Each cluster has a

\footnotetext{
${ }^{4} I_{i, \mu \nu}=\sum_{j \in \mathcal{N}_{i}} m\left(\delta_{\mu \nu} \mathbf{r}_{j}^{2}-r_{j, \mu} r_{j, \nu}\right)$.
} 

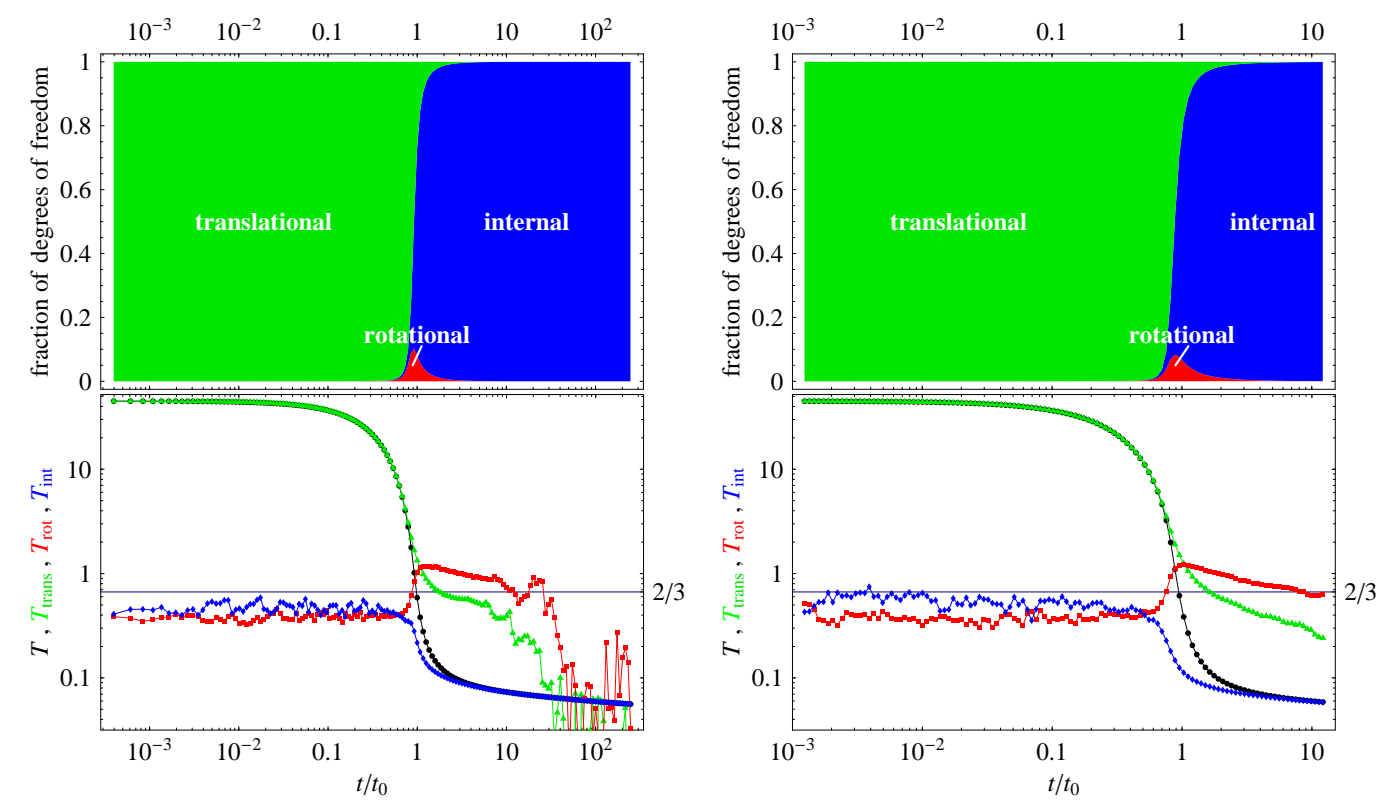

Figure 5.11: Top: Division of the total $3 N$ degrees of freedom into the translational, rotational and internal parts, dependent on time. Bottom: Evolution of the total $(\bullet)$, translational $(\boldsymbol{\Lambda})$, rotational $(\mathbf{\square})$, and internal $(\checkmark)$ granular temperatures. Data for $N=262144$ particles and volume fraction $\phi=1.95 \%$ (left) and $\phi=0.12 \%$ (right) are shown; the behavior is qualitatively the same for all investigated system sizes. The horizontal line at $2 / 3$ corresponds to the bond breaking energy.

total of $3 m_{i}$ degrees of freedom, so that the remaining number for internal degrees of freedom is $f_{\text {int }, i}=3 m_{i}-f_{\text {trans }, i}-f_{\text {rot }, i}$. The internal temperature $T_{\text {int }}$ is:

$$
T_{\mathrm{int}}:=\frac{2}{\sum_{i=1}^{n_{\mathrm{cl}}} f_{\mathrm{int}, i}} \sum_{i=1}^{n_{\mathrm{cl}}} E_{\mathrm{int}, i} .
$$

Fig. 5.11 (top) shows how the total of $3 N$ degrees of freedom divide up into translational, rotational, and internal degrees of freedom. The corresponding temperatures are shown in the lower half of the figure. As one might expect, for $t \ll t_{0}$ almost all degrees of freedom are translational, since most clusters are just single particles, and $T_{\text {trans }} \approx T$. Keeping in mind that two particles are only defined as neighbors if their relative velocity is not sufficient to break the bond, only stable clusters (mostly dimers) enter the internal and rotational temperatures, and therefore $T_{\text {rot }}, T_{\text {int }}<\frac{2}{3} \Delta E=\frac{2}{3}$ for $t / t_{0}<1 .{ }^{5}$

In the transitional regime $t \approx t_{0}$, when the number of intermediate size clusters increases, the rotational degrees of freedom become important. Larger objects can have higher rotational energies without rupture ${ }^{6}$, therefore the growing clusters

\footnotetext{
${ }^{5}$ The factor $\frac{2}{3}$ is due to the relation $\frac{2}{3} \bar{E}_{\text {kin }}=T$ between temperature and energy.

${ }^{6}$ Roughly speaking, the maximum rotational energy $E_{\text {rot,max }}$ of a cluster with radius $r$ and mass $M$ is $E_{\mathrm{rot}, \max } \sim M r^{2} \omega^{2}$, where the maximum rotational frequency $\omega$ is limited by the centrifugal
} 
obtain energy from caught particles, and thus $T_{\text {rot }}$ increases until reaching the value of $T_{\text {trans }}$. After that, the energy of the incoming lumps is not sufficient to increase $T_{\text {rot }}$ any further.

In contrast to the homogeneous cluster rotations, the internal degrees of freedom which have higher energies than $\Delta E$ will in most cases result in a bond rupture, independent of the cluster size. Therefore, $T_{\text {int }}$ decreases monotonically. At late times $t \gg t_{0}$, large clusters have formed, thus almost all degrees of freedom are internal and $T \approx T_{\text {int }}$.

\subsection{Aggregation}

When the average kinetic energy per particle is comparable to the bond breaking energy, $t \approx t_{0}$, the system starts to form aggregates, which seem to grow in a self-similar process. In the following we are going to analyze these aggregates and compare them to cluster-cluster aggregation models [Jullien \& Botet, 1987]. As time proceeds, larger and larger clusters are formed. A spanning or percolating cluster is observed for all finite densities (provided the system size $L$ is large enough), and ultimately all particles and clusters have merged into a single cluster.

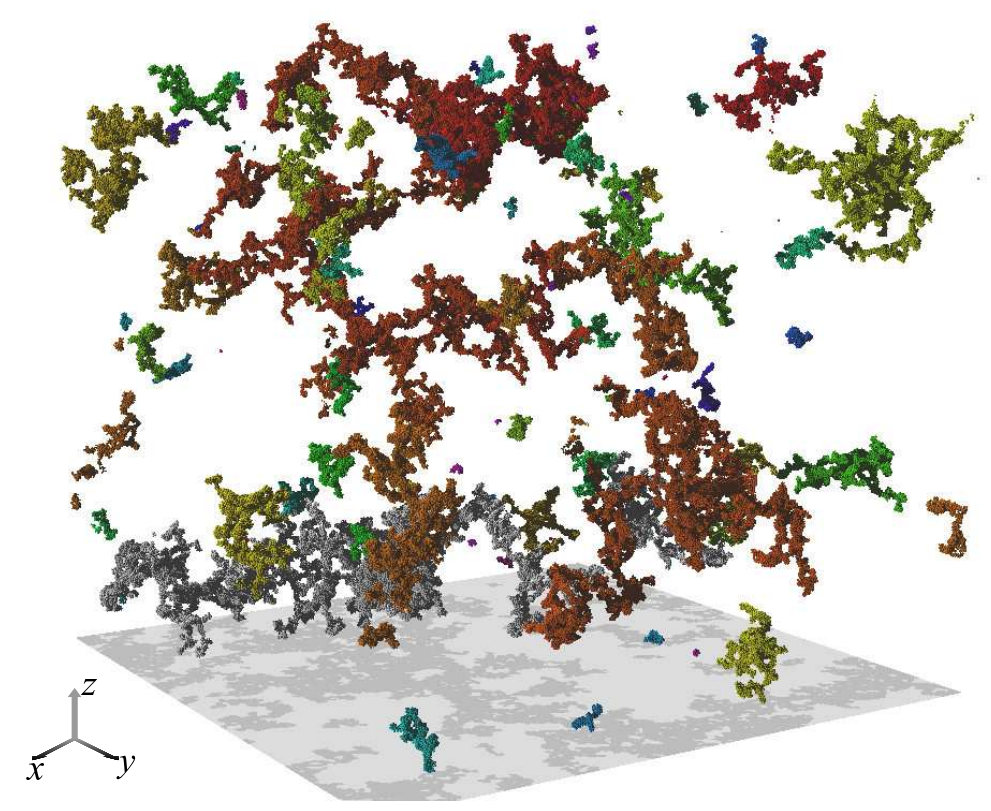

Figure 5.12: Snapshot of the system with volume fraction $\phi=0.48 \%$ and $N=$ 262144 particles taken at time $t \approx 12 t_{0}$; the largest cluster (gray) contains $22 \%$ of the particles. Particles of the same cluster have the same color shade.

force $F \sim M \omega^{2} r$. This yields $E_{\mathrm{rot}, \max } \sim r$. In our case the bond breaking energy $\Delta E$ is related to the maximum force $F$ on the particles by $\Delta E \sim F \cdot\left(d_{\mathrm{c}}-d\right)$, with the freely movable distance of a particle $\left(d_{\mathrm{c}}-d\right)$. 


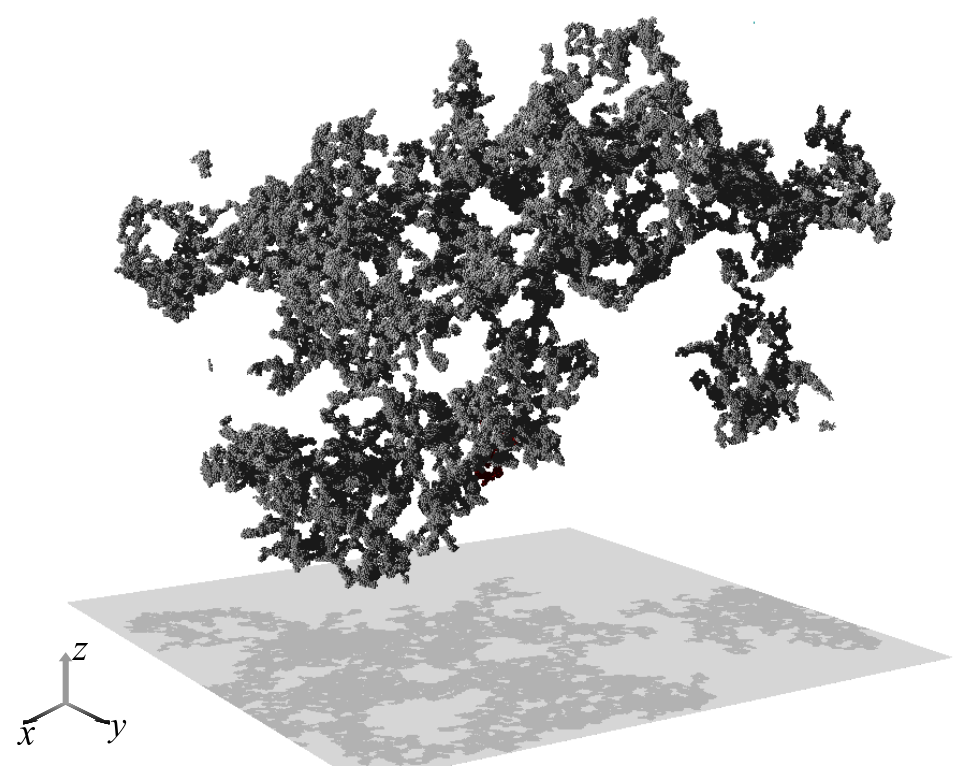

Figure 5.13: Same as Fig. 5.12 for $t \approx 52 t_{0}$; the largest cluster contains $99 \%$ of the particles.

Figs. 5.12 and 5.13 show snapshots of a system at $t=12 t_{0}$ and $t=52 t_{0}$ with small volume fraction, $\phi=0.48 \%$. At the smaller time the system is not yet percolating, even though rather large clusters have already formed, the largest one (in gray) contains $22 \%$ of all particles. The second snapshot, taken at a much longer time, shows a spanning cluster. At such large times the average kinetic energy is much smaller than the bond breaking energy $(T \approx 0.06 \Delta E)$, so that bonds almost never break up. The cluster shown is already well beyond the critical time for percolation with $99 \%$ of the particles in the cluster.

Fig. 5.14 shows the evolution of the cluster mass distribution $N_{m}(t)$, which is the number of clusters containing $m$ particles at time $t$. One can clearly see that after some time, $t \approx 2.5 t_{0}$, which depends on volume fraction, the largest cluster emerges from the rest of the distribution. For all volume fractions a gelation transition was observed at the percolation time $t_{c}>t_{0}$. The critical behavior of the gelation transition is still controversial. Since aggregation is a nonequilibrium process, there is a priori no reason that it should be in the same universality class as the corresponding equilibrium percolation transition. Yet there is some evidence in favor of this conjecture. Gimel et al. [1995] observe a cross-over from self-similar growth at small times and volume fractions - called the flocculation regime - to the percolation regime around $t_{c}$. In the latter they observe critical exponents ${ }^{7}$ as in standard percolation theory. Kolb \& Herrmann [1985] on the other hand obtain values for the fractal dimension of the percolating cluster, distinct from percolation

\footnotetext{
${ }^{7}$ like fractal dimension, or scaling of the mean cluster mass with the distance from the critical concentration
} 
theory as well as from flocculation theory. Both studies refer to diffusion limited cluster-cluster aggregation.

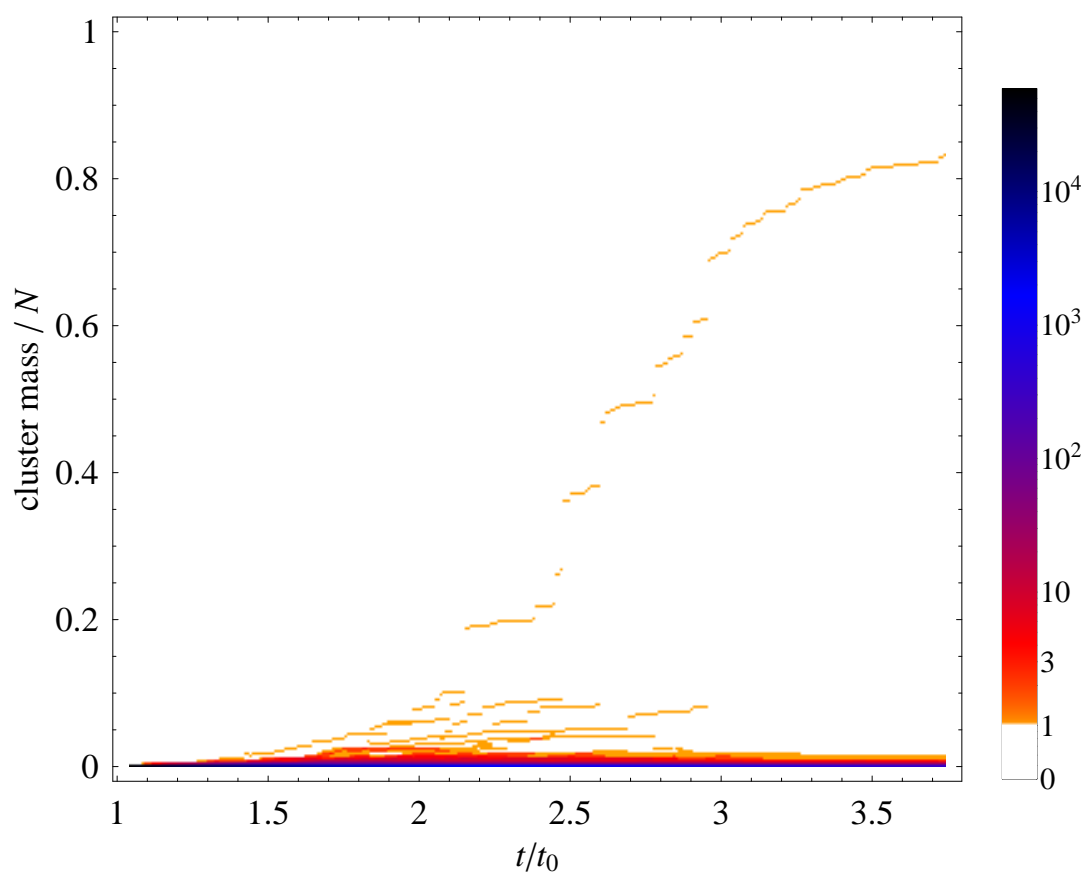

Figure 5.14: Histogram of the cluster mass distribution dependent on time, for volume fraction $\phi=3.9 \%$ and $N=262144$. The number of clusters at the respective time and size is color coded on a logarithmic scale so that the single largest cluster is visible. At $t \approx 2.5 t_{0}$ one can see the large cluster emerging, clearly distinguishable from the rest of the distribution.

Here we do not analyze the gelation transition in detail, however, work along these lines is in progress. Instead we investigate two regimes in detail in the following:

a) The self-similar growth process, or flocculation regime, which is present for small times and volume fractions.

b) The properties of the final cluster which emerges, when (almost) all particles have aggregated to form one large cluster.

\subsubsection{Self-Similar Growth}

\subsubsection{Fractal Dimension of the Aggregates}

A central quantity of aggregation models is the fractal dimension of the aggregates. It is usually determined from the radius of gyration as a function of cluster mass. We consider a cluster of $m$ particles with positions $\left(\mathbf{r}_{1}, \ldots, \mathbf{r}_{m}\right)$ and define its radius 
of gyration by [see e.g. Stanley \& Ostrowsky, 1986]

$$
r_{\mathrm{g}}^{2}(m)=\frac{1}{m} \sum_{i=1}^{m}\left(\mathbf{r}_{i}-\overline{\mathbf{r}}\right)^{2} \quad \text { with } \quad \overline{\mathbf{r}}=\frac{1}{m} \sum_{i=1}^{m} \mathbf{r}_{i} .
$$

If the clusters are fractal we expect a scaling relation for large $m$ of the form

$$
r_{\mathrm{g}} \sim m^{1 / D_{\mathrm{f}}}
$$

which yields the fractal dimension $D_{\mathrm{f}}$. This method is commonly used in aggregation models, where particles move diffusively, ballistically, or are interacting and stick to the aggregate once they touch it [Jullien \& Kolb, 1984; Meakin, 1991; Westbrook et al., 2004; Alves \& Ferreira, 2006].

Fig. 5.15 shows the radius of gyration for a system of 262144 particles at volume fraction $\phi=1.95 \%$. Several snapshots of the ensemble of growing clusters have been taken at times $t_{0}<t<t_{c}$ with the percolation time $t_{c}$, when a spanning cluster is first observed. The data scale well according to Eq. (5.34), some scatter is observed for the largest masses, corresponding to times close to the percolation transition.

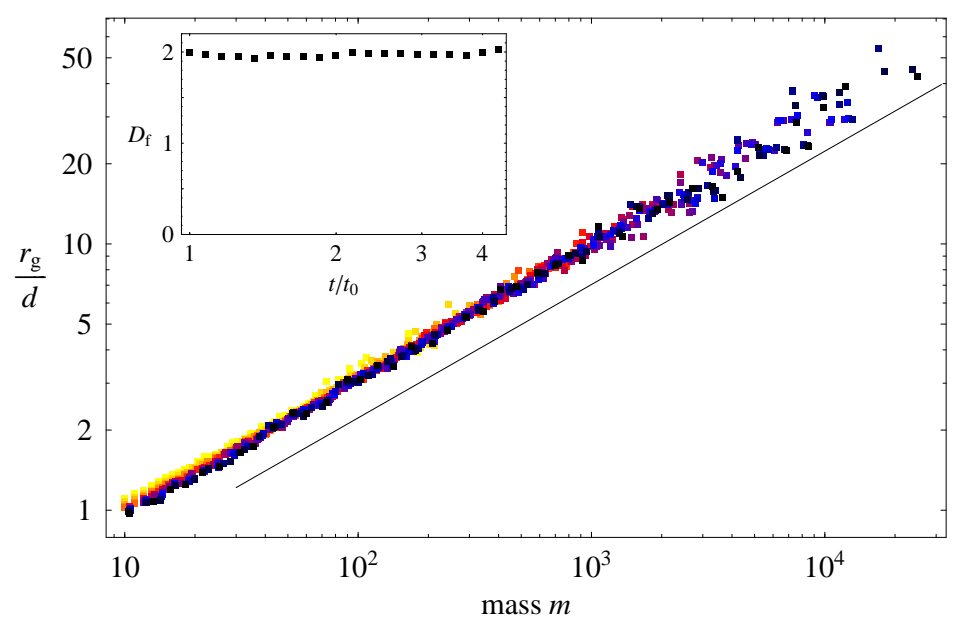

Figure 5.15: Radius of gyration as a function of cluster size for a system of 262144 particles at volume fraction $\phi=1.95 \%$; different colors/shades correspond to simulation times between $t_{0}$ (yellow) and $4 t_{0}<t_{c}$ (black)); The slope of the solid line corresponds to $D_{\mathrm{f}}=2$. Inset: fractal dimension as a function of time, extracted from the slope of the curves in the main figure.

In contrast to aggregation models, where the clusters are static and do not break up, we occasionally do observe the breaking of bonds. In addition there are internal deformations of the clusters during growth, so that the fractal dimension could depend on time. Therefore the relation between $m$ and $r_{\mathrm{g}}(m)$ is checked for many instances of time and the resulting fractal dimension is shown as a function of time in the inset of Fig. 5.15. As can be seen from the Figure, there is no systematic dependence on time, and the fractal dimension is close to $D_{\mathrm{f}}=2$. 


\subsubsection{Cluster Mass Distribution}

All information about the connectivity of the clusters is contained in the cluster mass distribution $N_{m}(t)$, the number of clusters of size $m$ at time $t$. Fig. 5.16 shows $N_{m}(t)$ for a system with $\phi=1.96 \%$ and $N=1048576$. The time interval has been chosen such that $t_{0}<t<2 t_{0}<t_{c} \approx 4 t_{0}$ (for this volume fraction). In this time interval the mean cluster mass increases roughly by a factor of 30 .

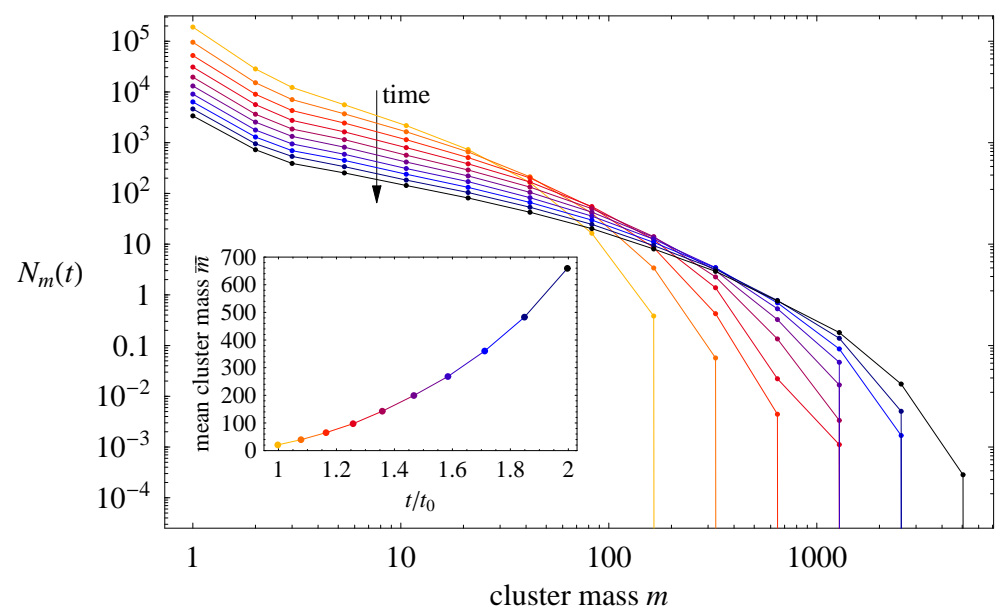

Figure 5.16: The cluster mass distribution $N_{m}(t)$. The different graphs represent different times, which are increasing from top to bottom (left side of the graph). The inset shows how the mean cluster mass increases during the investigated time period, where the colors correspond to the times of the main plot.

It has been suggested [e.g. Meakin, 1991] that for aggregating systems the mass distribution evolves towards a self-preserving scaling form, independent of the initial distribution:

$$
N_{m}(t)=m^{-\theta} f(m / \bar{m}(t)),
$$

where the time dependence is only contained in the mean cluster mass

$$
\bar{m}(t)=\frac{\sum_{m=1}^{\infty} m^{2} N_{m}(t)}{\sum_{m=1}^{\infty} m N_{m}(t)} .
$$

This scaling form has been applied successfully to various aggregating systems [Vicsek \& Family, 1984; Botet \& Jullien, 1984; Jiang \& Leyvraz, 1993, 1994; van Dongen \& Ernst, 1985; Meakin, 1991; Trizac \& Hansen, 1995], involving fractal as well as non-fractal objects. Mass conservation requires $\theta=2$ [Meakin, 1991].

In Fig. 5.17 the scaling function $f(m / \bar{m})=N_{m}(t) m^{2}$ is plotted for the same data sets as in Fig. 5.16. We expect scaling to hold only in the aggregation regime, i.e. for times not too close to $t_{c}$, where the system gels (see next Sec. 5.4.1.3); that is why we restrict ourselves to times $t_{0}<t<2 t_{0}$. Also the data points for $m=1$ have been left out, i.e. clusters consisting of single particles. As can be seen from 
Fig. 5.17 the data scale very well. Deviations occur only for times close to the percolation transition (not shown here), where they should be expected.

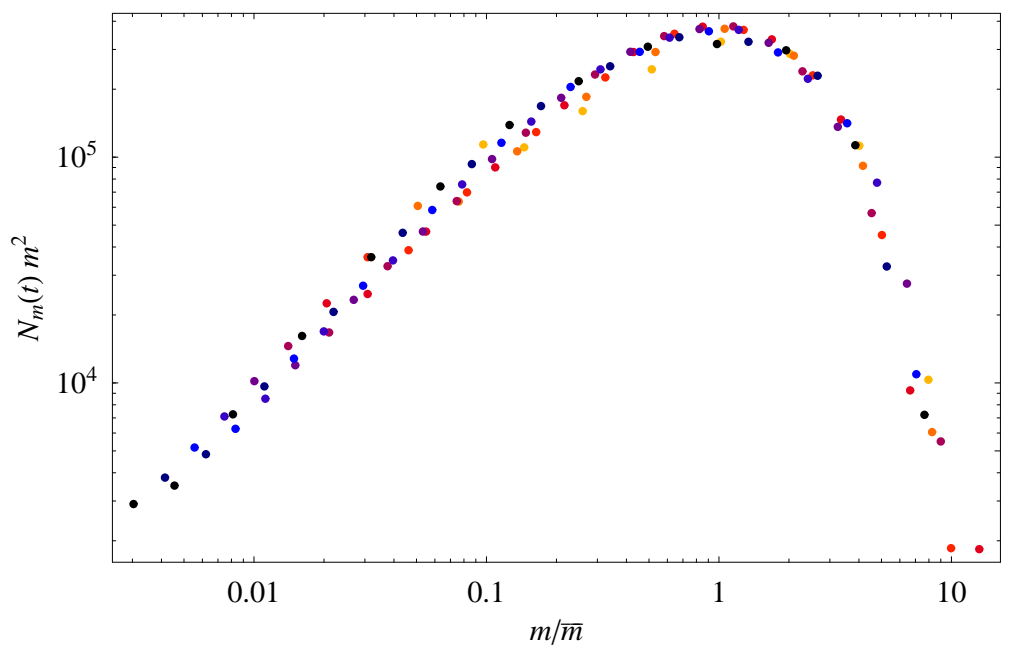

Figure 5.17: Rescaled cluster mass distribution $f(m / \bar{m})=N_{m}(t) \cdot m^{2}$ from Eq. (5.35) versus the normalized cluster mass $m / \bar{m}$. The color coding as in Fig. 5.16 is used.

\subsubsection{Number of Clusters and Mean Cluster Mass}

Another characteristic of a realization of clusters is simply the total number of clusters $n_{\mathrm{cl}}(t)=\sum_{m=1}^{\infty} N_{m}(t)$, which decreases as aggregation proceeds. As long as the system is in the scaling regime (i.e. relation (5.35) is fulfilled), the mean cluster mass, $\bar{m}(t)$ and the number of clusters are simply related: $\bar{m}(t) \sim n_{\mathrm{cl}}^{-1}$. However, as mentioned above, the scaling relation (5.35) only holds in the aggregation regime and is expected to break down as the percolation transition is approached. At that point, $\bar{m}$ should diverge due to the formation of a spanning cluster. On the other hand, there is still a large number of smaller clusters coexisting with the macroscopic cluster, so that $n_{\mathrm{cl}} / N$ remains finite at the percolation transition.

The aggregation of particles to larger objects has been investigated for various ballistic aggregation models [Family \& Vicsek, 1985; Carnevale et al., 1990; Jiang \& Leyvraz, 1994; Trizac \& Krapivsky, 2003], where spherical particles of mass $m=1$ and diameter $d=d_{0}$ move ballistically, until two of them collide to form clusters irreversibly. In a particularly simple model, one assumes that two colliding particles form one larger spherical particle with conserved momentum and a mass $m$ equal to the sum of the two particles masses, so that $m$ is always equal to the number of initial particles contained in a given cluster. For spatial dimension $D$, the diameter increases like $d=m^{1 / D} d_{0}$, assuming the particles to be compact spheres which conserve volume when merging. For this model, a mean field theory [Jiang \& Leyvraz, 1994] and simple scaling arguments [Carnevale et al., 1990; Trizac \& Krapivsky, 2003] yield the dependence of the expected average mass $\bar{m}$ on time like $\bar{m} \sim t^{\xi}$ 
with an exponent $\xi=2 D /(D+2)$ (assuming $t_{0}=0$ ).

Since the aggregating clusters in our system are not compact, but fractal objects with fractal dimension $D_{\mathrm{f}}$, the assumption for the diameter $d \sim m^{1 / D}$ does not hold and must be changed to $d \sim m^{1 / D_{\mathrm{f}}}$. With this assumption, we follow the scaling arguments of Trizac \& Krapivsky [2003], and find the scaling relation between $\bar{m}$ and $t$.

We assume that the number of clusters per volume, $n_{\mathrm{cl}}$, is reduced by one whenever two clusters collide:

$$
\frac{\mathrm{d} n_{\mathrm{cl}}}{\mathrm{d} t} \sim-f_{\mathrm{coll}} \cdot n_{\mathrm{cl}} \cdot
$$

The collision frequency [Brilliantov \& Pöschel, 2004] is approximately given by $f_{\text {coll }} \sim d^{D-1} n_{\mathrm{cl}} v$ with $d \propto r_{\mathrm{g}}$ the linear dimension of the cluster and $v$ its typical velocity. The average momentum should scale as $p \sim m^{1 / 2}$ [Trizac \& Krapivsky, 2003], and therefore

$$
v=p / m \sim m^{-1 / 2} \sim n_{\mathrm{cl}}^{1 / 2} .
$$

Plugging in all these scaling relations as well as $m \sim r_{\mathrm{g}}^{D_{\mathrm{f}}}$, one obtains:

$$
\frac{\mathrm{d} n_{\mathrm{cl}}}{\mathrm{d} t} \sim-n_{\mathrm{cl}}^{2} \cdot v \cdot d^{D-1} \sim-n_{\mathrm{cl}}^{5 / 2-(D-1) / D_{\mathrm{f}}},
$$

which is solved by

$$
n_{\mathrm{cl}} \sim\left(t-t^{*}\right)^{-2 D_{\mathrm{f}} /\left(3 D_{\mathrm{f}}-2 D+2\right)},
$$

where the integration constant $t^{*}$ is the onset of cluster growth. In our context $t^{*} \approx t_{0}{ }^{8}$ This implies the following growth law for the mean cluster mass in the scaling regime:

$$
\bar{m} \sim\left(t-t^{*}\right)^{\xi} \quad \text { with } \quad \xi=\frac{2 D_{\mathrm{f}}}{3 D_{\mathrm{f}}-2 D+2},
$$

which generalizes the result for compact objects, $\xi=2 D /(D+2)$ with $D=D_{\mathrm{f}}$ to fractal ones with $D \neq D_{\mathrm{f}}$.

Fig. 5.18 shows how the number of clusters decreases over time as larger and larger aggregates form for $t>t_{0}$. The inset of Fig. 5.18 investigates the scaling behavior (5.40), with the origin of the time axis shifted to the transition point $t^{*}$. One can see that the slope of $\xi=2$, obtained from (5.41) for $D=3$ and $D_{\mathrm{f}}=2$ is in good agreement with the simulation.

\subsubsection{Properties of the Asymptotic Cluster}

The largest cluster - well beyond the percolation transition for most volume fractions - will be the main focus of this section. In particular we determine its fractal dimensions and local structure. For the fractal dimension, there are various definitions, which in general may yield different results and three of which are presented. Particularly illuminative is the box counting algorithm: simple considerations make a prediction about a cross-over length scale at which the cluster structure changes from fractal to compact behavior, which is indeed observed in the simulation data.

\footnotetext{
${ }^{8}$ As one can see in the main plot of Fig. 5.18, the actual onset of cluster growth is not exactly at $t_{0}$, but a little bit earlier.
} 


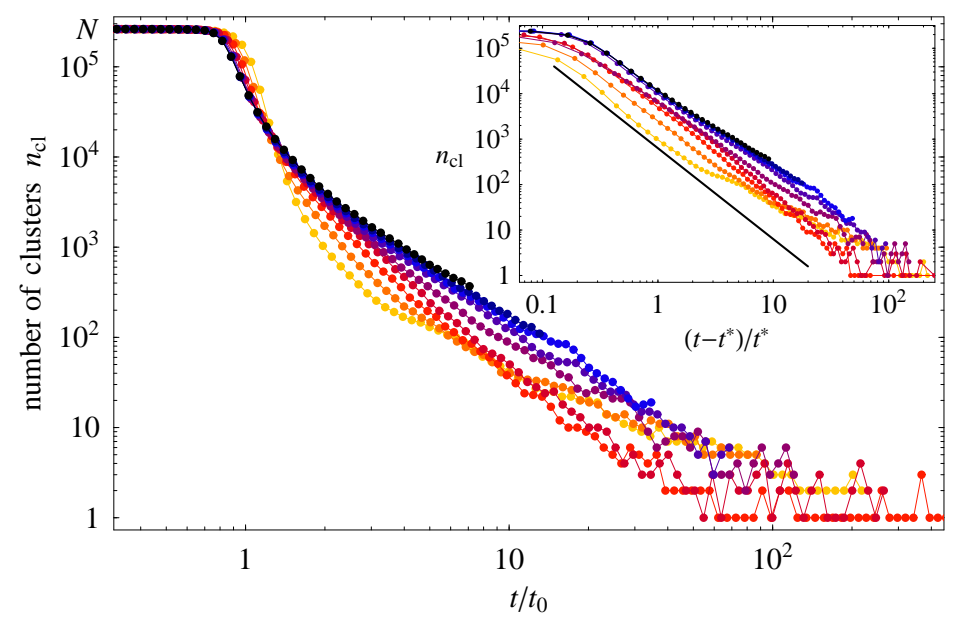

Figure 5.18: Evolution of the mean cluster mass. Labeling and parameters as in Fig. 5.3. For the inset, the origin of the time-axis has been shifted to the transition point $t^{*}$ to investigate the scaling relation $n_{\mathrm{cl}} \sim\left(t-t^{*}\right)^{-\xi}$. The solid line has a slope of -2 .

\subsubsection{Fractal Dimension from Radius of Gyration}

One way to determine the fractal dimension is the radius of gyration, as was done in Sec. 5.4.1.1 for aggregates. Here, however, we only have one large cluster and have to find a way to obtain the function $r_{\mathrm{g}}(m)$ as a function of cluster size $m$. This is implemented in following way: Starting from a random particle of the cluster, we mark all particles that can be reached through $i$ or less neighbor-to-neighbor steps (see Fig. 5.19). Thus, for every $i$, we get a partial cluster with $m(i)$ particles and radius of gyration $r_{\mathrm{g}}(i)$, which yields the scaling relation $r_{\mathrm{g}} \sim m^{1 / D_{\mathrm{f}}}$ and the fractal dimension $D_{\mathrm{f}}$. For good statistics, this procedure is repeated 100 times (each with a different initial particle) and the obtained values of $r_{\mathrm{g}}$ are averaged. Note furthermore that the procedure takes care that no particle is marked a second time, in order to make sure that one does not go through the cluster several times because of the periodic boundary conditions.

Fig. 5.20 shows the results of this procedure for the radius of gyration $r_{\mathrm{g}}$ as a function of $m$ for different densities. For high volume fractions we are well beyond the percolation transition and hence expect $D_{\mathrm{f}}=3$ on the largest length scales of the cluster. This is clearly seen in Fig. 5.20, e.g. for $\phi=15.6 \%$ and $10^{3}<m<10^{5}$. On smaller length scales, however, we find a fractal dimension $D_{\mathrm{f}} \approx 2$. For smaller volume fractions, the cross-over to $D_{\mathrm{f}}=3$ happens at larger masses and hence the fractal behavior extends to larger scales. In the following section we will see, why there must be a cross-over from fractal to compact behavior and how the volume fraction determines the cross-over point. 


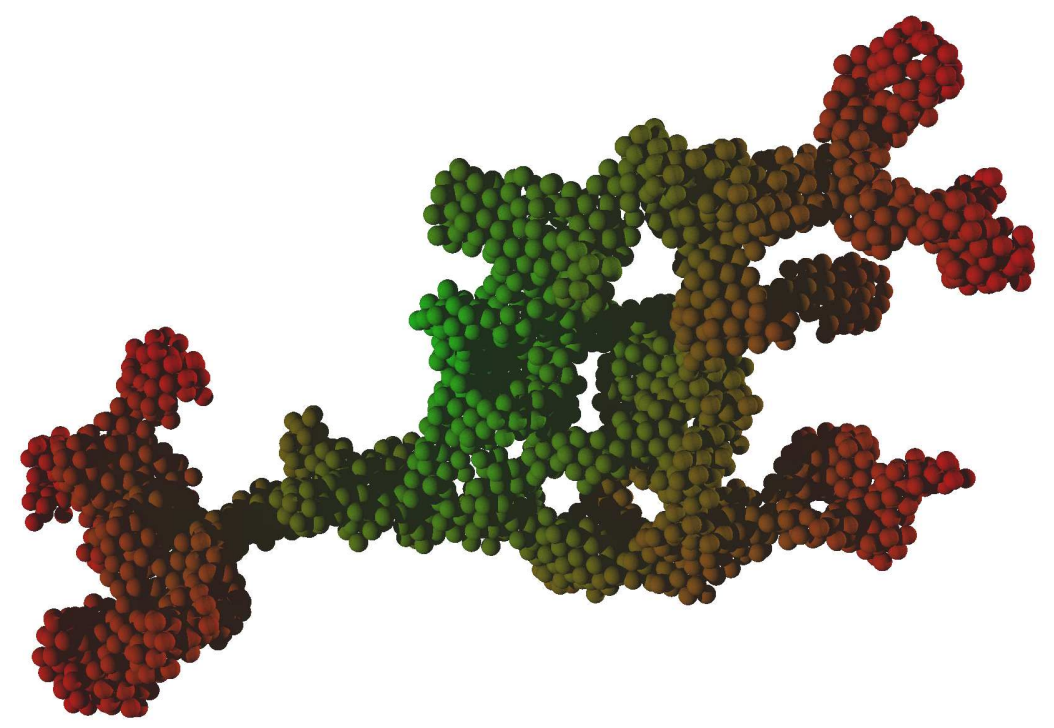

Figure 5.19: Illustration of the procedure to obtain partial clusters. The initial particle is marked in pure green. The number of neighbor-to-neighbor steps $i$ necessary to reach another particle is indicated by the color shade of the particles: Larger $i$ corresponds to a gradual shift towards red. The outermost particles (fully red) correspond to $i=36$ steps.

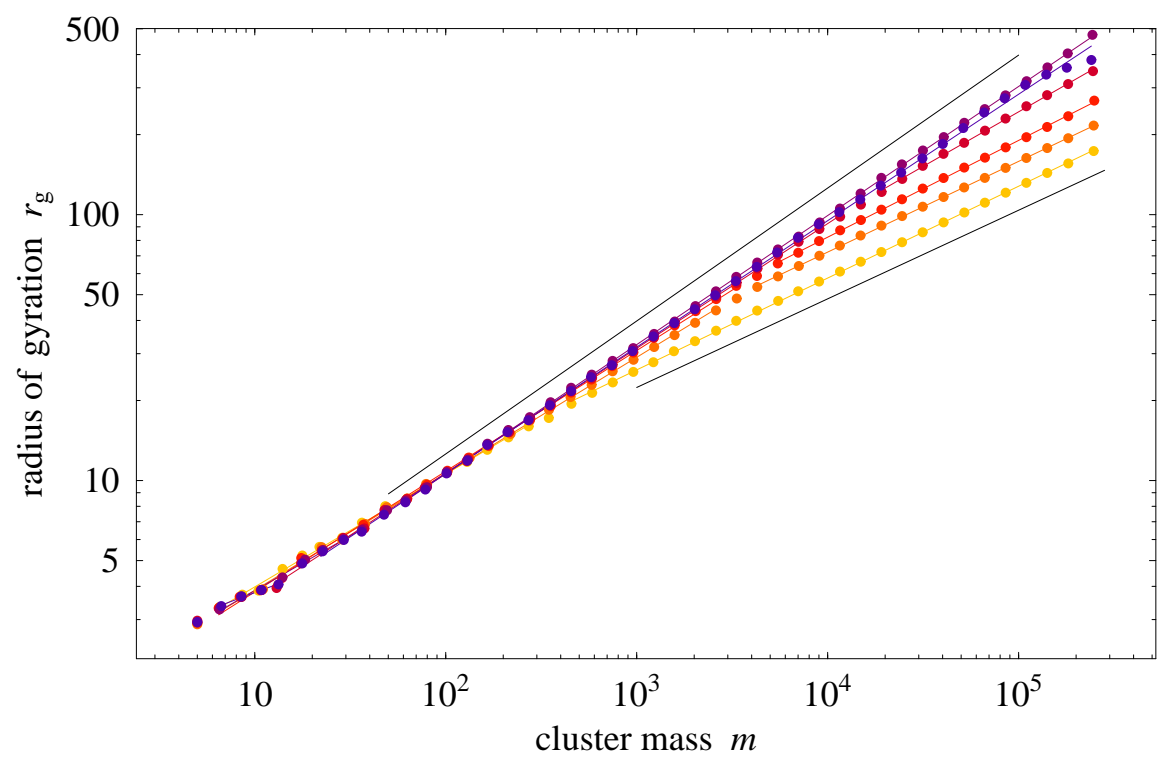

Figure 5.20: Radius of gyration dependent on the mass of the partial cluster at simulation time $t \approx 27 t_{0}$. The particle number is fixed $N=262144$ and the volume fractions are (from bottom to top) $\phi=15.6 \%, 7.81 \%, 3.90 \%, 1.95 \%, 0.98 \%, 0.49 \%$. The lines along the data points are the respective fits. The outer solid lines have slopes $1 / 2$ (top) and $1 / 3$ (bottom) corresponding to fractal dimensions of 2 and 3 , respectively. 


\subsubsection{Fractal Dimension from Box Counting Algorithm}

To further investigate the fractal dimension of the largest cluster at intermediate length scales, we use the box counting algorithm [Grassberger, 1983; Hentschel \& Procaccia, 1983]. The system is divided into sub-boxes of edge length $L_{\mathrm{box}}$. Then each box which contains or hits at least one particle is marked. In this way, we find the number of boxes $N_{\text {box }}$ necessary to cover the whole cluster. This number should scale with $L_{\text {box }}$ like

$$
N_{\text {box }} \sim L_{\text {box }}^{-D_{\mathrm{f}}},
$$

with the Hausdorff dimension $D_{\mathrm{f}}$.

On length scales much smaller than the particle diameter, $L_{\mathrm{box}} \ll d$, the system obviously behaves three-dimensionally. In this regime, the number of filled boxes $N_{\text {box }}$ is just the volume fraction $\phi$ times the total number of boxes $N_{\text {box,tot }}=$ $L^{3} / L_{\text {box }}^{3}$, therefore:

$$
N_{\text {box }}=\frac{\phi L^{3}}{L_{\text {box }}^{3}}
$$

Since our system is finite and contains a system-spanning cluster, the scaling behavior on large length scales $L_{\text {box }} \approx L$ should also be three dimensional. On this length scale, almost all the boxes should be filled, so that

$$
N_{\text {box }}=\frac{L^{3}}{L_{\text {box }}^{3}}
$$

In particular, the relation must include the point $\left(L_{\mathrm{box}}, N_{\mathrm{box}}\right)=(L, 1)$, since a box of the system size includes all particles and will certainly be marked.

Only in the regime between these two limiting cases is it possible to observe the fractal dimension with the box-counting method. A schematic plot is given on the left side of Fig. 5.21, where the number $N_{\text {box }}$ of boxes containing particles is plotted against the edge length $L_{\text {box }}$ of a box. Comparing (5.43) and (5.44) shows that the interesting range is proportional to $|\log \phi|$, which only depends on the volume fraction, but not on the particular choice of the system size.

For numerical reasons, it is very tedious to observe the expected slope of -3 for small $L_{\mathrm{box}}$, because of the vast amount of boxes to account for. Since this regime is not relevant anyway, it has only been investigated exemplarily and is reached for $L_{\text {box }} \lesssim 0.03 d$ (see right side of Fig. 5.21). For all other runs we simplify the algorithm and only use the centers of the particles, i.e. a box is only marked, if a particle center is inside. With this definition the number of boxes needed to cover the system for small box sizes $L_{\text {box }} \lesssim d$ is just the particle number $N$, resulting in a horizontal line on the left side of the graph (dotted line in Fig. 5.21), instead of the slope -3 .

Fig. 5.22 (top) shows the outcome of the box-counting algorithm, at a time $t \approx 27 t_{0}$, where roughly all particles are inside the largest cluster. It yields the relation between the box size $L_{\text {box }}$ and the number of boxes of that size, needed to 

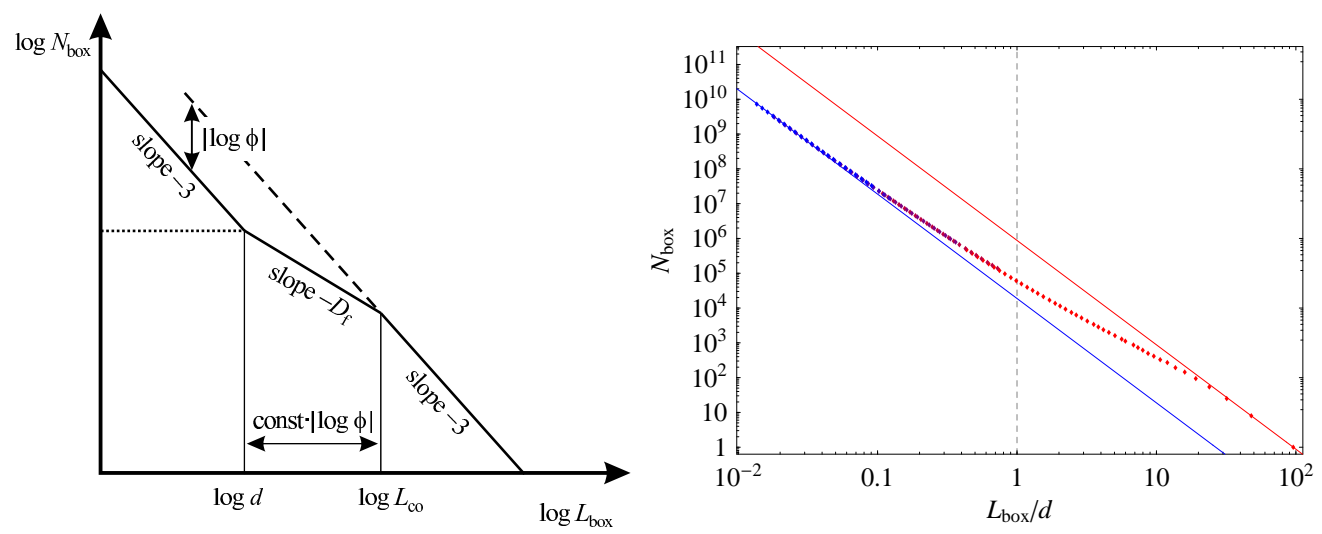

Figure 5.21: Left: Schematic double logarithmic plot of the box size $L_{\mathrm{box}}$ versus the number of boxes $N_{\text {box }}$ of that size needed to cover the cluster. The negative slope is the fractal dimension. We expect three scaling regions: For small and large $L_{\text {box }}$, the system should behave three dimensionally, and the region in between yields the non-trivial fractal dimension. If only the particle centers are considered, the algorithm simply counts the number of particles in the cluster for $L_{\text {box }} \lesssim d$ resulting in a horizontal line (dotted line). Right: Exemplary application of the box counting algorithm, considering the extent of the spheres. Only a small system ( $N=32768$ particles and $\phi=1.95 \%$ ) was used and on small length scales, only fractions of the system were investigated and the resulting data was rescaled: the different colors of the data points correspond to cubic sections of the whole system with edge length $L / 30, L / 24, L / 8, L / 4$, and $L$ (whole system). The solid lines are fits to the data points on the largest and smallest length scales. As proposed, their vertical distance of 1.67 roughly equals $\left|\log _{10} \phi\right|=1.71$.

cover the cluster. The slope of that curve is the negative fractal dimension. The result for different system sizes, but with the same volume fraction are presented. As proposed, for all system sizes, there is a cross-over point $L_{\mathrm{co}}$, at which the slope changes. On length scales between $d$ and $L_{\mathrm{co}}$, the fractal dimension is roughly 2 (the fits yield values between 1.92 and 2.03). Above $L_{\text {co }}$ the fractal dimension has a trivial value of about 3 (fit values between 2.95 and 3.00), which means that on these large length scales all the boxes are filled and is therefore an indication that the cluster is system-spanning.

In the lower half of Fig. 5.22 we see the number of boxes normalized by the cluster mass. The data collapse well onto a single curve, obviously with the same slopes. Here one can see very well that for systems with the same volume fraction, the slopes as well as the cross-over point do not depend on the absolute system size.

Results of the box counting algorithm for different densities are presented in Fig. 5.23 (top). Only densities for which a spanning cluster has developed are included. The slopes of -2 and -3 in the two scaling regions are not affected by the volume fraction $\phi$, but the size of the non-trivial region (with $D_{\mathrm{f}} \approx 2$ ) is seen to increase significantly as the density decreases. Even for the lowest density, the 


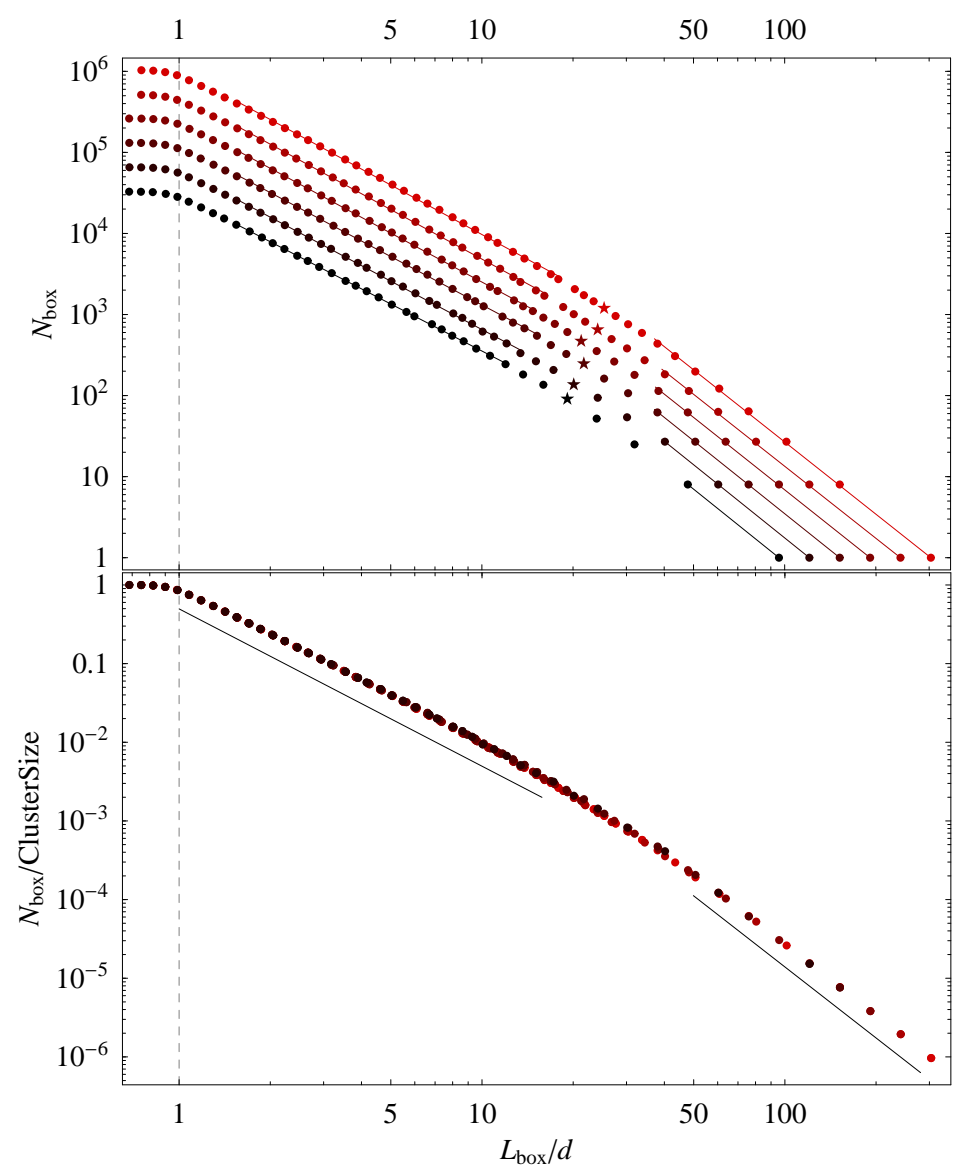

Figure 5.22: Top: $N_{\text {box }}$ versus $L_{\text {box }}$ at time $t \approx 27 t_{0}$ and volume fraction $\phi=1.95 \%$ for the box counting algorithm; particle number is varied from bottom to top, according to $N=32768,65536,131072,262144,524288,1048576$. The straight lines are fits to the data to the left and right of the cross-over point $L_{\mathrm{co}} \approx 25 d$, which is also a fitting parameter and shown as a star ( $\star$ ). Bottom: As top, but $N_{\text {box }}$ normalized by cluster mass; the solid lines have slopes -2 (left) and -3 (right); the vertical dashed line represents the particle size.

size of the scaling region is less than 2 decades, which makes it difficult to extract precise values for the fractal dimension. For the three most dense systems, the scaling region is less than one decade. As discussed earlier, this is an intrinsic feature of the "high" density systems, which can not be resolved by taking larger systems $(N, L \rightarrow \infty$ with constant $\phi)$. As the bottom panel of Fig. 5.23 shows, we can collapse all data on a single curve by rescaling $N_{\text {box }}$ with $\phi^{-2}$ and $L_{\text {box }}$ with $\phi$ in agreement with the dependence of the cross-over length on $|\log \phi|$. 

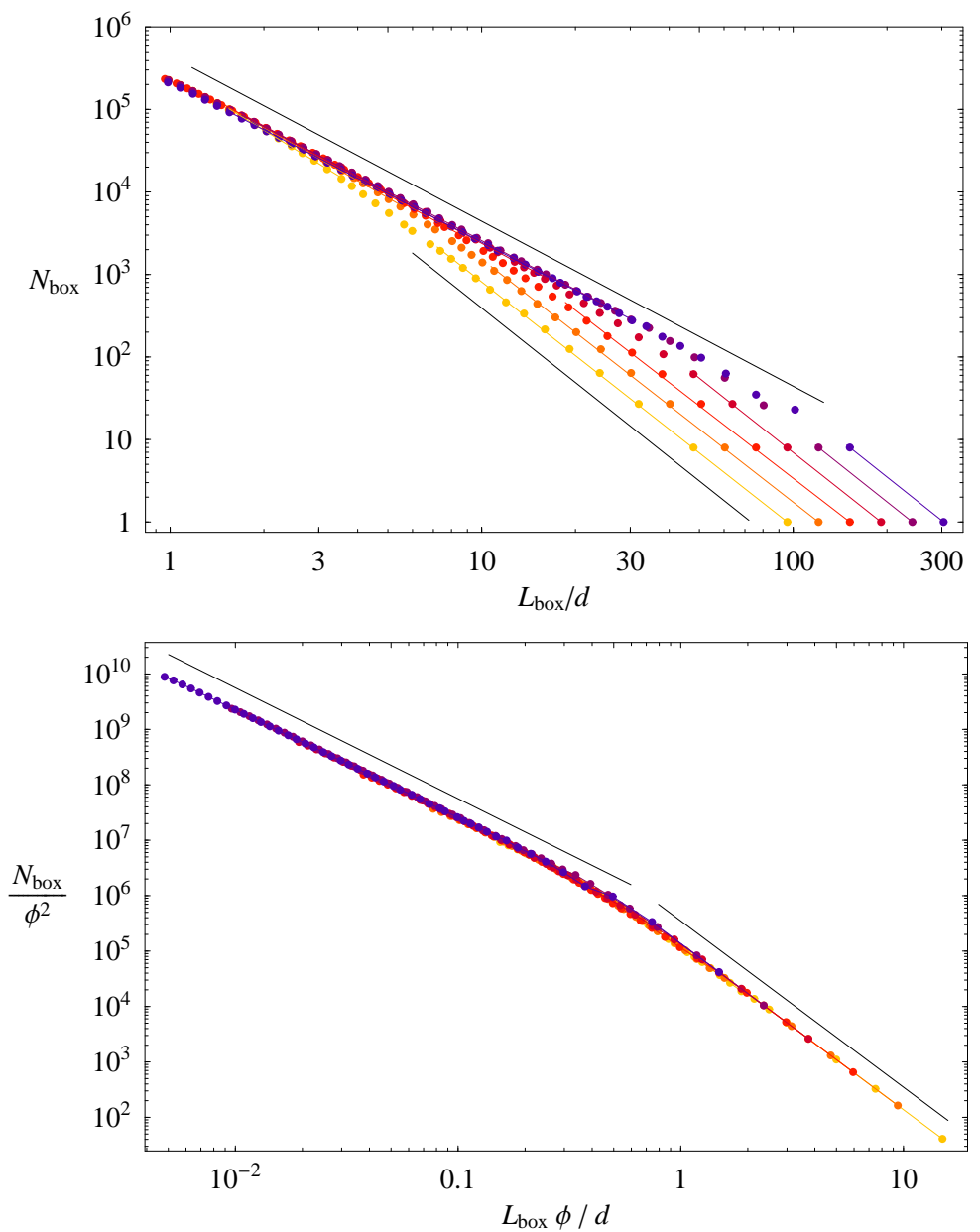

Figure 5.23: Top: Result of the box counting algorithm, as in Fig. 5.22 at $t \approx 45 t_{0}$; the particle number is fixed $N=262144$ and the volume fraction varies from left to right according to $\phi=15.6 \%, 7.81 \%, 3.90 \%, 1.95 \%, 0.98 \%, 0.49 \%$; the lines along the data points are the respective fits; the outer lines have slope -2 (upper line) and -3 (lower line). Bottom: As top, but with rescaled axes. 


\subsubsection{Pair Correlation Function and Correlation Dimension}

A fundamental quantity for granular (or atomic) systems is the pair correlation function $g(\mathbf{r})$ which specifies the probability that the centers of two particles are separated by a vector $\mathbf{r}$ [Hansen \& McDonald, 1986]:

$$
g(\mathbf{r})=\frac{1}{n}\left\langle\frac{1}{N} \sum_{i \neq j} \delta\left(\mathbf{r}_{i}-\mathbf{r}_{j}-\mathbf{r}\right)\right\rangle .
$$

Since the interactions of the particles do not give rise to anisotropies, one typically only considers the radial component, i.e. the radial distribution function (RDF) $g(r)$; specifically, it is the average of $g(\mathbf{r})$ over all directions of $\mathbf{r}$ but with fixed $|\mathbf{r}|=r$, and describes how the particle density varies as a function of the distance from a given particle. Due to the normalization with the particle density $n, g(r) \rightarrow 1$ for $r \gg d$, if there are no long range correlations. Furthermore, since hard spheres cannot overlap, obviously $g(r)=0$ for $r<d$.

In between these two limits, $g(r)$ can give information about the structure of the system. If e.g. there is short range order and close-by particles arrange in small lattice structures, $g(r)$ shows peaks at distances corresponding to a few next neighbor steps of the respective lattice.

In Fig. 5.24 the radial distribution function is shown for a system with $N \approx 10^{6}$ and $\phi=1.96 \%$ at different times. For details on the computation of $g(r)$, see Appendix D.3. As a consistency check, we confirm that the simulation data for the (equilibrated) start configuration (Fig. 5.24(a): $t=0$ ) agrees with the well known Percus-Yevick solution for hard spheres [Boon \& Yip, 1991], an approximation for $g(r)$ that is presumably excellent for low volume fractions.

For $t \approx 3 / f_{\text {coll }}$, Fig. 5.24(b), each particle had on average three collisions, and the temperature $T \approx 44 \Delta E$ is still much higher than $\Delta E$. However, one can already see the effect of the attractive bonds, since $g(r)$ has significantly increased beyond 1 between $d$ and $d_{\mathrm{c}}$. This observation has also been made for similar systems with hard core repulsion, but energy conserving square-well potential: For this system, Dawson et al. [2000] also find a $g(r)$ much larger than 1 for $d<r<d_{\mathrm{c}}$, with discontinuous jumps at $d$ and $d_{\mathrm{c}}$.

During the cooling regime, as long as $T \gg \Delta E$, this graph remains qualitatively the same, with an increasing area under the curve between $d$ and $d_{\mathrm{c}}$. As the transition to the clustering regime is approached (Fig. 5.24(c), $t \approx t_{0}$ and $T \approx \Delta E$ ) and larger stable clusters emerge, peaks arise in $g(r)$ also for $r>d_{\mathrm{c}}$, corresponding to second and further next neighbor distances in the clusters. 
(a) $t=0$

(b) $t \approx 3 / f_{\text {coll }}$

(d) $t \approx t_{\mathrm{c}}$ (c) $t \approx t_{0}$

(e) $t=t_{\max }$

Figure 5.24: Radial distribution function $g(r)$ for a system with $N=1048576$ particles and volume fraction $\phi=1.96 \%$. In (a), the equilibrated start configuration is shown and compared to the Percus-Yevick solution (blue) for $g(r)$. In (b), $t \approx$ $3 / f_{\text {coll }}$ and the temperature $T / \Delta E \approx 44 \gg 1$. In (c), data is shown for $t \approx t_{0}$, the transition point between cooling and clustering regime, with $T / \Delta E \approx 1$. In (d), $t \approx t_{\mathrm{c}}$, i.e. a percolating cluster has emerged for the first time. And in (e), the latest simulated time is shown, in which the largest cluster contains $99.6 \%$ of all particles. In (a)-(c), all particles are considered; in (d) and (e) data for all particles (green) and for the percolating cluster only (blue) are superimposed, but they are virtually identical in the shown region of small $r$. The vertical red lines in (b)-(e) show the hard core distance $d$, the bond breaking distance $d_{\mathrm{c}}$, and the second nearest neighbor distance $d_{\mathrm{HCP}}$ of a hexagonal close packed structure. The dashed horizontal gray line in all plots corresponds to $g(r)=1$. 
In Fig. 5.24(d), $g(r)$ is shown at the percolation time $t_{\mathrm{c}} \approx 4 t_{0}$, at which a systemspanning cluster has developed. Here one can see very well that the correlations extend further than the next-neighbor distance. The first peak for $r>d_{\mathrm{c}}$ appears at the second nearest neighbor distance of the hexagonal closed packed lattice $d_{\mathrm{HCP}}=$ $\frac{1}{2} \sqrt{3} \cdot \frac{d+d_{\mathrm{c}}}{2} ;{ }^{9}$ the further peaks appear in accordance with other lattice distances. For the green and blue curve, respectively, all particles and only particles of the percolating cluster are considered; however, there seems to be no difference in the shown "short range" structure $(r \leq 3 d)$, as the two curves are almost identical.

Fig. 5.24(e) shows $g(r)$ at the latest simulated time $\left(t \approx 40 t_{0}\right)$, again considering all particles (green) and only the percolating cluster (blue), which now contains $99.6 \%$ of all particles. One can see that the area under the curve between $d$ and $d_{\mathrm{c}}$ has marginally increased, but other than that there are no significant differences to (d), suggesting that the characteristics of the local structure has not significantly changed on the small length scales $\left(d_{\mathrm{c}}<r \leq 3 d\right)$, even though the simulation time has increased by a factor of 10 and extensive restructuring of the cluster has taken place.

On length scales much larger than the particle diameter, the RDF can give information about the dimensionality of the respective structure. Let us, for example, consider a planar object embedded in a three dimensional system, and have a look at some point on this object. A volume element at distance $r$ from this point is less and less likely to contain a part of that object, as $r$ is increased; this is because, the surface of a sphere grows with its radius $r$ like $r^{2}$, but the intersection of that sphere with the planar object only like $r$; hence $g(r)$ should fall of like $\frac{r}{r^{2}}=\frac{1}{r}$. In this regard, one defines the so called correlation dimension (which we also denote $D_{\mathrm{f}}$ ) [Grassberger, 1983; Rottereau et al., 2004] for fractal objects by

$$
g(r) \propto r^{D_{\mathrm{f}}-D} \quad \text { for } \quad r \gg d .
$$

As we already noted before, for a structure with homogeneous density (i.e. $\left.D_{\mathrm{f}}=D\right)$, we get $g(r) \rightarrow 1$ in the limit of large $r$.

Fig. 5.25 shows the decay of $g(r)$ in the large $r$ regime for the final cluster of the same system as above. Consistent with the fractal behavior with previous definitions of the fractal dimension (Secs. 5.4.2.1 and 5.4.2.2), there appears to be a cross-over at about $r \approx 20 d$ from the fractal behavior on smaller length scales to a compact behavior on larger length scales. Due to the oscillations of $g(r)$ up to about $r=5 d$ and the large cross-over region, the regime with well-defined slope only covers half a decade, which makes the precise identification of $D_{\mathrm{f}}$ impossible. Nevertheless this regime appears to be consistent with a fractal dimension of $D_{\mathrm{f}}=2$ (blue line), the result of the box-counting and the radius-of-gyration method.

Beyond the cross-over point, we expect the structure to be compact, which would result in a horizontal progression of $g(r)$. However, due to the finite system size, $g(r)$ is eventually cut off, hence the cross-over to a horizontal line cannot be observed well. As we found in Sec. 5.4.2.2, the size of the fractal regime is proportional to

\footnotetext{
${ }^{9}$ We assumed that the lattice constant is the mean between $d$ and $d_{\mathrm{c}}$.
} 
Figure 5.25: Radial distribution function $g(r)$ for the same system as in Fig. 5.24, however, in the regime $r \gg d$. The blue line has a slope of -1 corresponding to a correlation dimension $D_{\mathrm{f}}=2$. The system size is $L=304 d$. The inset shows data of the final cluster for a system with higher density $\phi=7.8 \%$ and $N=2097152$ particles.

$|\log \phi|$ and hence should decrease as the density $\phi$ is increased. Thus, the inset of Fig. 5.25 shows data for a denser system with $\phi=7.8 \%$ and $N \approx 2 \cdot 10^{6}$, where the cross-over to compact behavior can be observed well. 


\subsubsection{Coordination Number}

Given the definition of a neighborhood relation (two particles are neighbors if they have built a bridge and their kinetic energy is not sufficient to break it), we can extract the average number of neighbors of a particle, i.e. the average coordination number. A good understanding of the development of the system can be obtained from the evolution of the coordination numbers. This is shown for the thick film model in Fig. 5.26. Here the clustering transition can be seen very well: Initially

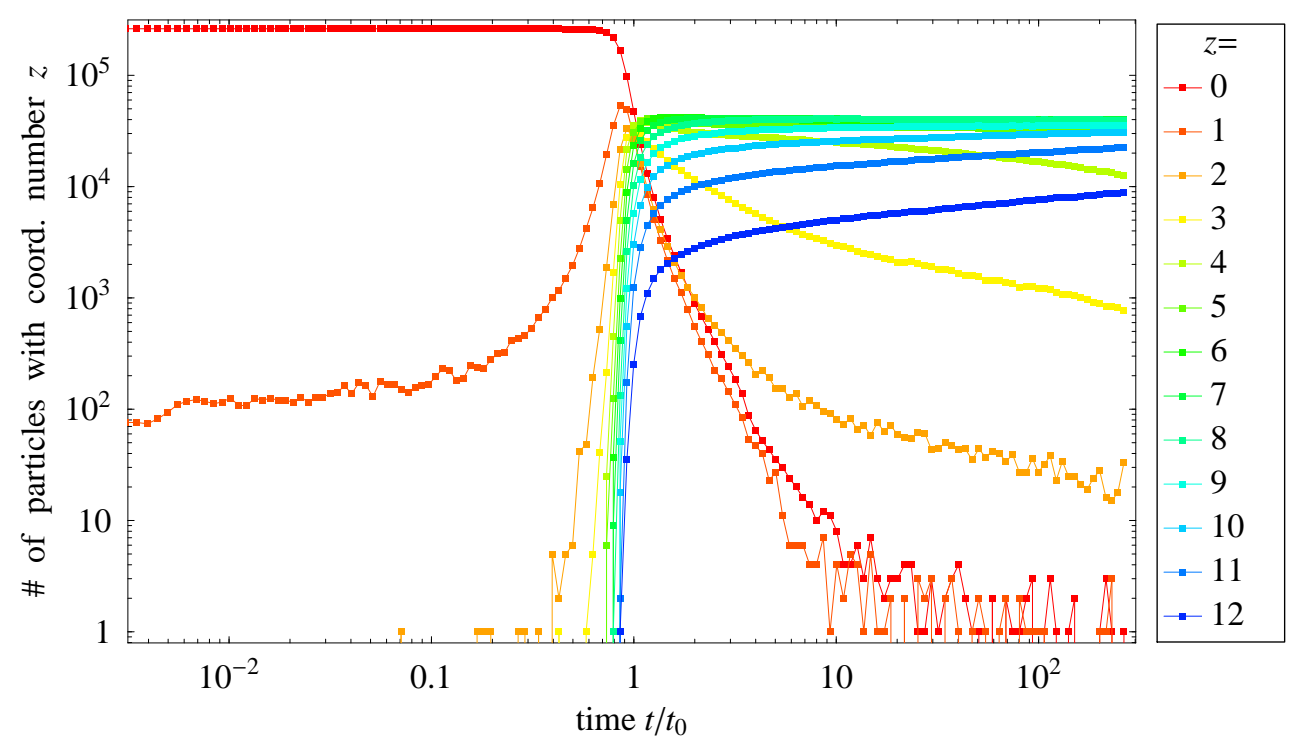

Figure 5.26: Time evolution of the coordination numbers, considering all particles. The data points show the number of particles with coordination number $z$, where different colors correspond to different $z$, as indicated by the legend on the right. The system has $N=262144$ particles, volume fraction $\phi=1.95 \%$, and bond breaking distance $d_{\mathrm{c}}=1.07 d$.

mostly single particles are found, as the dominant coordination number is $z=0$. Only a small fraction appears as dimers $(z=1)$ and larger aggregates are negligible. At the transition $t=t_{0}$, larger coordination numbers $z=2,3,4, \ldots$ increase sharply, one after another, indicating that larger aggregates are forming. After this sharp increase at the transition point, a coordination number of about $z \approx 6$ is most prevailing. As time proceeds smaller coordination numbers slowly diminish, and larger ones slowly continue to increase.

Let us now have a closer look at the nature of the asymptotic cluster: Fig. 5.27 (top) shows histograms for the coordination number in the percolating cluster for the thick film model with two different bond-breaking distances. As one would expect, these distributions are rather broad with coordination numbers between 
one and twelve ${ }^{10}$. The smaller bond-breaking distance (left) gives rise to a more asymmetric distribution with more weight for smaller coordination numbers. The bottom panel of Fig. 5.27 shows the typical histogram of coordination numbers for the thin film model. As we can see, the dependence on $d_{\mathrm{c}}$ is weaker for the thin film model and the asymmetry for small $d_{\mathrm{c}}$ is less pronounced.
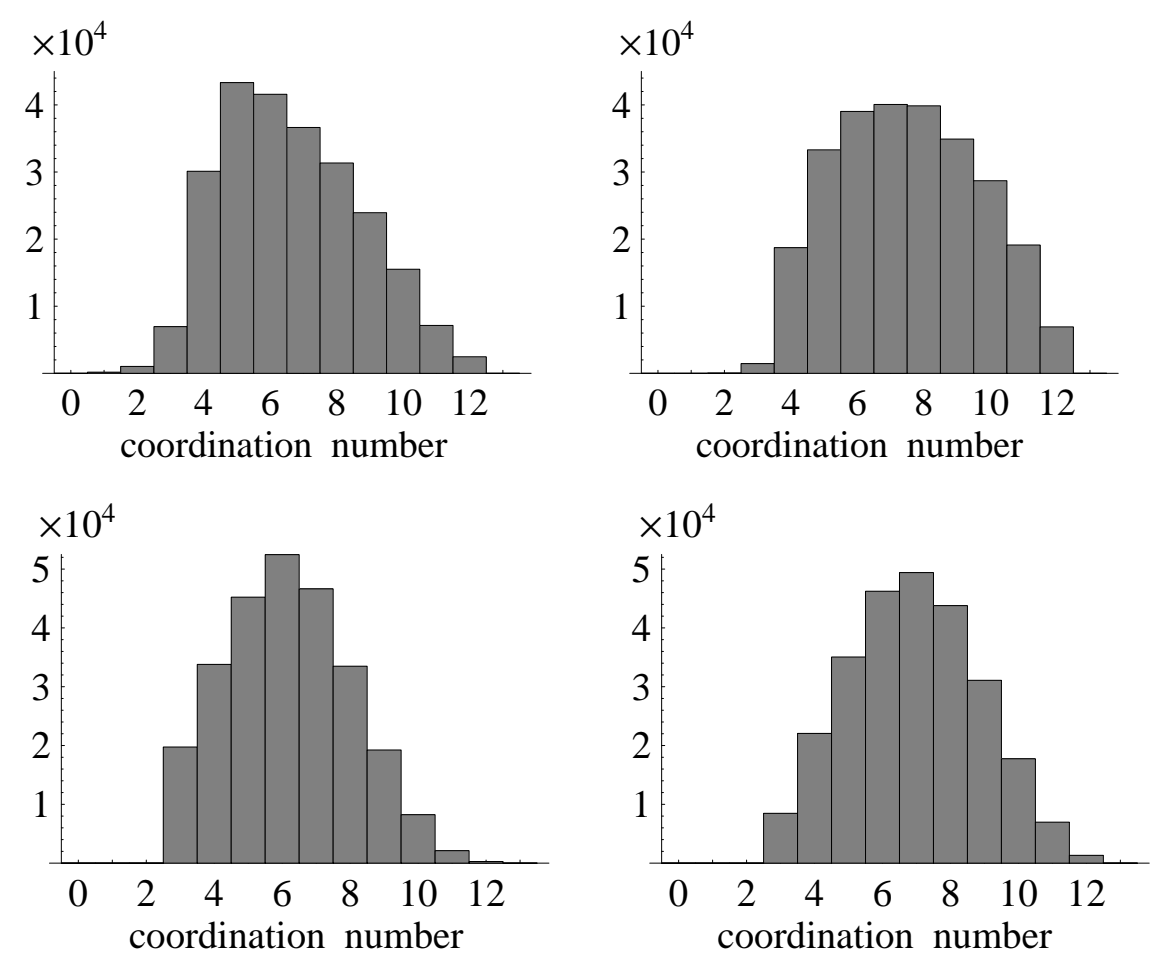

Figure 5.27: Top: Histogram of the coordination number of the asymptotic cluster for two different bond breaking distances $d_{\mathrm{c}}=1.01 d$ (left) and $d_{\mathrm{c}}=1.07 d$ (right); both plots show data from the thick film model with $N=262144$ and $\phi=1.95 \%$. Bottom: As top, but for the thin film model.

More comprehensible than the full histogram is the average coordination number of the largest cluster, whose time evolution is shown in Fig. 5.28 for different bond-breaking distances $d_{\mathrm{c}}$. After a strong increase at the time $t_{0}$, the average coordination number continues to grow slowly. This slow increase is strongly suppressed in the thin film model (right) as compared to the thick film model (left). Within the thin film model the slow growth with time is further suppressed for decreasing bond-breaking distance $d_{\mathrm{c}}$.

As one can see in Figs. 5.27, 5.28, the coordination number becomes smaller for smaller $d_{\mathrm{c}}$. This is reasonable, because the particles can more easily collect neighbors for higher $d_{\mathrm{c}}$. As $d_{\mathrm{c}} \rightarrow d$ the average coordination number of the thin film model approaches 6 , which is the isostatic value. This is demonstrated in

\footnotetext{
${ }^{10}$ Very rarely, a coordination number of $z=13$ is observed for $d_{\mathrm{c}}=1.07 d$, however not in the presented system.
} 

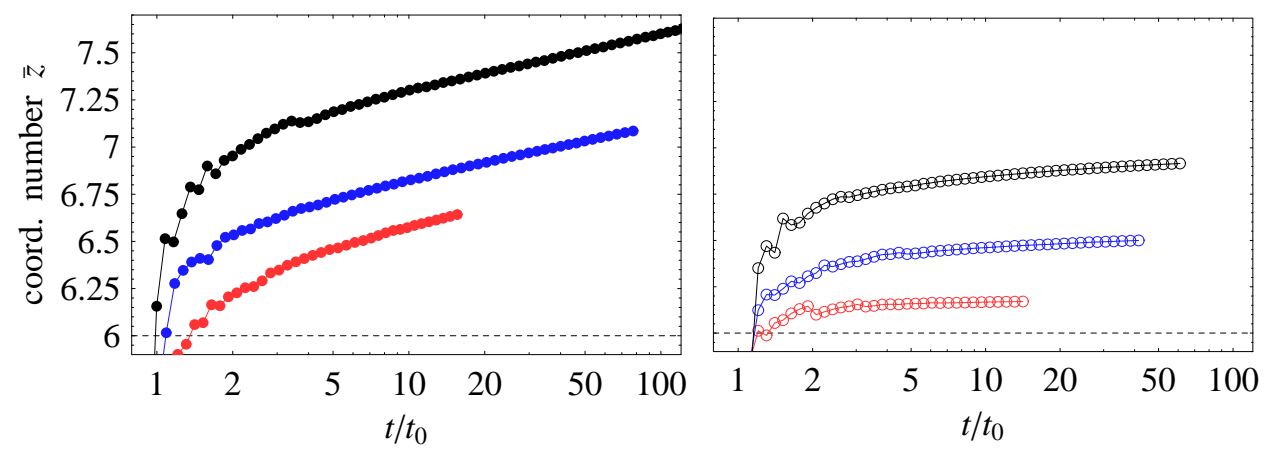

Figure 5.28: Time evolution of the average coordination number $\bar{z}$ of particles in the largest cluster $(N=262144$ and $\phi=1.95 \%)$; the critical break-off distances are $d_{\mathrm{c}}=1.07 d, 1.035 d$ and $1.01 d$ from top to bottom; thick film model (left) in comparison to thin film model (right).

Fig. 5.29, where we plot the asymptotic coordination number as a function of $d_{\mathrm{c}}$. Here the asymptotic value is taken, when $T<0.06 \Delta E$ for the first time.

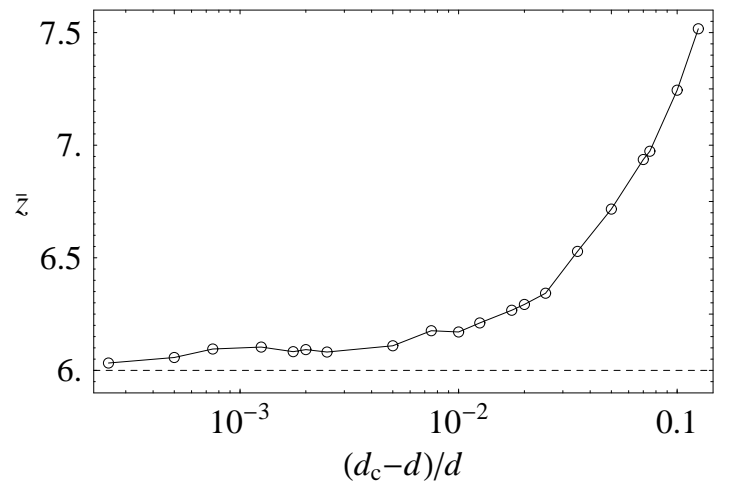

Figure 5.29: Influence of the bond-breaking distance $d_{\mathrm{c}}$ on the final value of the average coordination number $\bar{z}$ for the thin film model. $\phi=0.24 \%$ and $N=10648$.

Naively one might expect that the increase of the coordination number with larger $d_{\mathrm{c}}$ is caused by a compactification and therefore accompanied by an increase of the fractal dimension. However, as can be seen in Fig. 5.30, there is no significant influence of $d_{\mathrm{c}}$ on the development of the fractal dimension. Thus, we conclude that this compactification is mostly occurring on the single particle length scale and therefore increasing the average coordination number, but not influencing the structure on larger length scales. ${ }^{11}$

\footnotetext{
${ }^{11}$ Note that there is also a very slight increase of the fractal dimension and therefore also a very slow compactification on larger length scales. However these effects are much less pronounced than the change of the coordination number.
} 


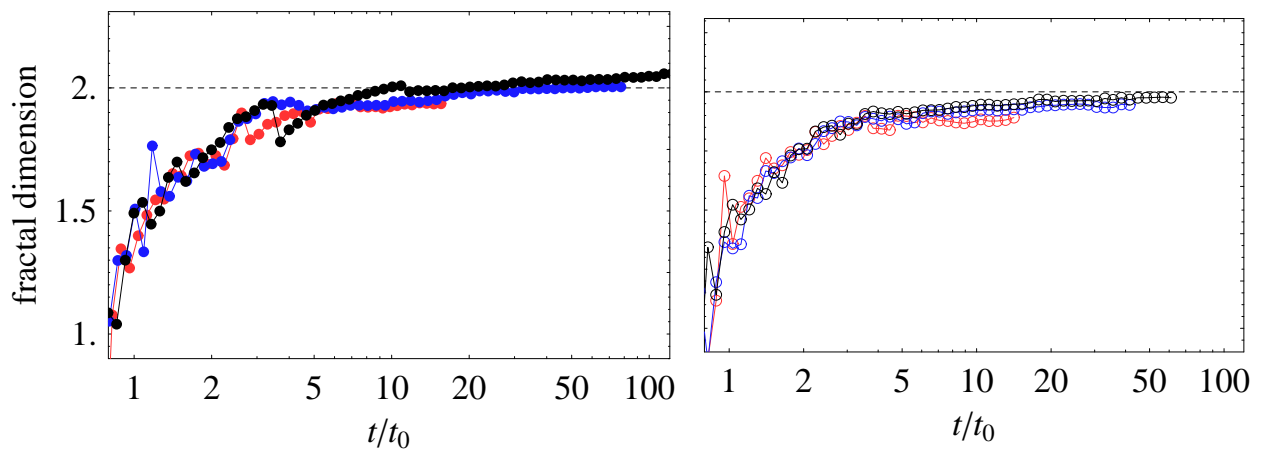

Figure 5.30: Time evolution of the fractal dimension $D_{\mathrm{f}}$ of the largest cluster. System and colors as in Fig. 5.28. 


\subsubsection{Inertia Tensor and Spatial Extension}

Throughout this section 5.4.2 we repeatedly observe a fractal dimension of $D_{\mathrm{f}} \approx 2$ for the asymptotic cluster; this raises the question, whether or not the cluster largely consists of planar topologies. To gain quantitative information about the shape of an object, it is useful to compute its moment of inertia tensor [see e.g. Landau \& Lifshitz, 1976] and look at its eigenvalues, the principal moments of inertia $I_{1} \leq I_{2} \leq I_{3}$. The latter quantify the mass distribution perpendicular to the respective principal axes $\mathbf{e}_{1}, \mathbf{e}_{2}, \mathbf{e}_{3}$, as they obey the equation:

$$
I_{1}=\int \varrho(\mathbf{r})\left(r_{2}^{2}+r_{3}^{2}\right) \mathrm{d} \mathbf{r},
$$

and equivalent equations for $I_{2}, I_{3}$. Here $\mathbf{r}=\sum_{\nu=1}^{3} r_{\nu} \mathbf{e}_{\nu}$ is the distance from the center of mass in the coordinate system of the principal axes and $\varrho(\mathbf{r})$ the density at that point.

The principal moments of inertia can easily be related to a more intuitively comprehensible quantity, the spatial extensions $\Delta r_{\nu}$ along the three principal axes:

$$
\Delta r_{\nu}^{2}:=\frac{\int \varrho(\mathbf{r}) r_{\nu}^{2} \mathrm{~d} \mathbf{r}}{\int \varrho(\mathbf{r}) \mathrm{d} \mathbf{r}} \quad \text { for } \quad \nu=1,2,3
$$

While the denominator is simply the mass of the object, the numerator can be expressed in terms of the principal moments; e.g. for $\nu=1$ :

$$
\begin{aligned}
\frac{-I_{1}+I_{2}+I_{3}}{2} & =\int \varrho(\mathbf{r}) \frac{-\left(r_{2}^{2}+r_{3}^{2}\right)+\left(r_{1}^{2}+r_{3}^{2}\right)+\left(r_{1}^{2}+r_{2}^{2}\right)}{2} \mathrm{~d} \mathbf{r} \\
& =\int \varrho(\mathbf{r}) r_{1}^{2} \mathrm{~d} \mathbf{r} .
\end{aligned}
$$

The reason for not calculating Eq. (5.48) directly, but using the detour via the principal moments of inertia, is that a priori the principal axes are not known. Note furthermore that from these spatial extensions one can trivially obtain the radius of gyration $r_{\mathrm{g}}^{2}=\sum_{\nu=1}^{3} \Delta r_{\nu}^{2}$, as one can easily see in the definition (5.48). The choice of the principal axes is always such that $\Delta r_{1} \geq \Delta r_{2} \geq \Delta r_{3}$. For spherical symmetric objects obviously $\Delta r_{1}=\Delta r_{2}=\Delta r_{3}$; for plate-like, or so-called oblate objects $\Delta r_{1} \approx \Delta r_{2} \gg \Delta r_{3}$, and for rod-like, or prolate objects $\Delta r_{1} \gg \Delta r_{2} \approx \Delta r_{3}$.

In order to obtain information about the structure on a wide range of length scales, we analyze partial clusters deduced from the largest cluster, as done in Sec. 5.4.2.1: starting from a random initial particle of the cluster, we define all particles that can be reached by $i$ or less neighbor-to-neighbor steps as partial cluster $\mathcal{N}(i)$ (see Fig. 5.19). The spatial extensions $\Delta r_{1,2,3}$ of $\mathcal{N}(i)$ are calculated for different values of $i$ and the data are averaged over several initial particles. ${ }^{12}$ The result is shown in Fig. 5.31. As one can see, the structures are clearly not spher-

\footnotetext{
${ }^{12}$ For simplicity only the particle centers are considered for the calculation of the inertia tensor. As we can easily convince ourselves in Eq. (5.49), due to the Huygens-Steiner theorem, accounting for the extension of the individual particles would just lead to a constant shift of $\Delta r_{1,2,3}^{2} \rightarrow \Delta r_{1,2,3}^{2}+$ $\frac{1}{2} I_{\text {sphere }}$ with the moment of inertia of a single sphere $I_{\text {sphere }}=\frac{1}{10} d^{2}$.
} 


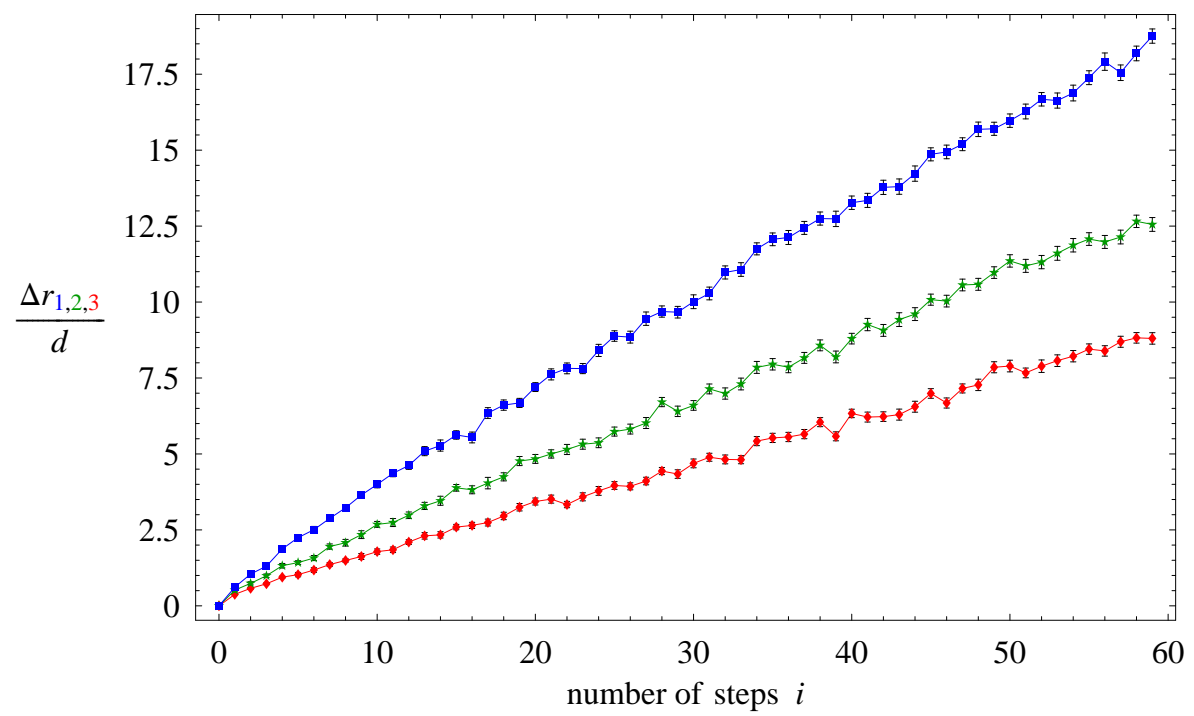

Figure 5.31: Spatial extension of partial clusters $\mathcal{N}(i)$ along the principal axes. The blue curve shows the largest component $\Delta r_{1}$, the green curve the second largest $\Delta r_{2}$, and the red curve the smallest $\Delta r_{3}$. The inset shows data for the same procedure, for which, however, the particle positions are not obtained from the simulation, but randomly placed into the system.

ically symmetric, as the spatial extensions in the principal directions are different for all shown length scales. However, also the assumption that the cluster involves planar structures cannot be supported, since that would imply $\Delta r_{1} \approx \Delta r_{2} \gg \Delta r_{3}$ at least on some intermediate length scales. Indeed, we observe the most general case $\Delta r_{1} \not \Delta \Delta r_{2} \not \approx \Delta r_{3}$ throughout the investigated range.

\subsection{Conclusions}

We have analyzed a simple model of a wet granulate allowing for large scale event driven simulations. A central feature of wet granulates is the existence of an energy scale $\Delta E$ associated with the rupture of a capillary bridge between two grains. This energy scale has important consequences not only for the phase diagram [Fingerle et al., 2008] but also for the free cooling dynamics investigated in this paper. The most important feature is a well defined transition at a time $t_{0}$, when the kinetic energy $T$ of the particles becomes equal to $\Delta E$.

For $t<t_{0}$ the particles are energetic enough to supply the bond breaking energy $\Delta E$, so that very few collisions result in bound pairs and most particles are unbound. Cooling is very effective in this regime, but drastically different from a dry granulate. Whereas in dry granulates the dissipated energy is proportional to the energy of the colliding particles, in wet granulates the dissipated energy is $\Delta E$, independent of the energy of the colliding particles so that $\dot{T} \propto \sqrt{T}$. Consequently Haff's law does 
not hold and is replaced by $T(t)=T(0)\left(1-t / t_{0}\right)^{2}$ for $t<t_{0}$. The simulations are in very good agreement with this cooling law for $t<t_{0}$.

For $t>t_{0}$, the kinetic energy of the particles is too small to provide the bond breaking energy, so that larger and larger clusters form. We call this regime the aggregation regime and analyze the properties of the aggregates. For not too long times and sufficiently small volume fractions, we observe flocculation characterized by nonoverlapping, weakly interacting clusters. The fractal dimension of the aggregates is $D_{\mathrm{f}} \approx 2$. The cluster mass distribution follows a simple scaling form, $N_{m}(t) \propto m^{-2} f(m / \bar{m}(t))$, which has been applied successfully to different aggregation models before. The increase of the typical cluster size $\bar{m}(t)$ can be understood by a simple scaling analysis: Assuming that clusters irreversibly stick together when they hit upon each other and that their radius $r$ grows with the number of particles $m$ like $r^{D_{\mathrm{f}}} \propto m$, yields a cluster growth $\bar{m} \propto t^{2 D_{\mathrm{f}} /\left(3 D_{\mathrm{f}}-2 D+2\right)}$. This scaling relation shows good agreement with the simulation for fractal dimension $D_{\mathrm{f}}=2$.

At larger times, a spanning cluster forms, and a gelation transition is observed for all finite volume fractions. At the gelation transition a spanning cluster coexists with many small ones, whereas at very long times almost all particles are connected to one large cluster. On the largest length scales the final cluster is no longer a fractal but compact, as one would expect for a spanning cluster in the percolating phase. On smaller length scales, however, we still find fractal structures with $D_{\mathrm{f}} \approx 2$. The range where a nontrivial fractal dimension can be observed increases with decreasing density as $|\log \phi|$.

Even on the longest time-scales, the temperature continues to decay. In this regime the limiting process is the breaking of a bond. The probability for this process becomes exponentially small $P_{\mathrm{bb}} \propto \sqrt{\Delta E / T} \mathrm{e}^{-\Delta E / T}$ as the temperature goes to zero. Hence the cooling law for high temperatures is replaced by $\dot{T} \propto \mathrm{e}^{-\Delta E / T}$, yielding a slow (logarithmic) temperature decay, which is in very good agreement with the data.

Several extensions of the presented work might be interesting. So far we have completely neglected all inelasticities except for the bond rupture. One expects the collisions at the hard core to be dissipative as they are in dry granular media. In the simplest model these could be described by normal restitution. Furthermore, real wet grains experience frictional forces, coupling translational and rotational motion of the grains [Brilliantov et al., 2007]. However, the author is not aware of any such studies for wet granulates at the present time. In the context of gelation it would be interesting to see, if and how a finite shear modulus emerges in the system, as the percolation transition is passed. 


\section{Properties of $\perp$ - and $\|$-vectors}

The properties of $\perp$ - and $\|$-vectors discussed here apply to the calculations in chapters 2 and 3 . For any given replicated vector $\hat{x}=\left(\mathbf{x}^{(0)}, \ldots, \mathbf{x}^{(n)}\right)^{T}$, define:

$$
\mathbf{x}_{\|}:=\frac{\sum_{\alpha=0}^{n} \mathbf{x}^{(\alpha)}}{\sqrt{n+1}}
$$

and

$$
x_{\perp}:=\left(\begin{array}{c}
\mathbf{x}^{(0)} \\
\vdots \\
\mathbf{x}^{(n)}
\end{array}\right)-\frac{1}{n+1}\left(\begin{array}{c}
\sum_{\alpha=0}^{n} \mathbf{x}^{(\alpha)} \\
\vdots \\
\sum_{\alpha=0}^{n} \mathbf{x}^{(\alpha)}
\end{array}\right)=\hat{x}-\frac{\mathbf{x}_{\|} \otimes \hat{\varepsilon}}{\sqrt{n+1}}
$$

with $\hat{\varepsilon}=(1, \ldots, 1)^{T}$. Then these vectors have the following properties:

- The mean of all components of $x_{\perp}$ is $\mathbf{x}_{\perp}^{(0)}+\ldots+\mathbf{x}_{\perp}^{(n)}=\mathbf{0}$

- Therefore $\left(\mathbf{x}_{\|} \otimes \hat{\varepsilon}\right) \cdot x_{\perp}=0$

- $\hat{x} \cdot \hat{y}=\mathbf{x}_{\|} \cdot \mathbf{y}_{\|}+x_{\perp} \cdot y_{\perp}$

When integrating over a function $f(\hat{x})=f\left(\mathbf{x}_{\|}, x_{\perp}\right)$, we might ask the the question how to change integration variables

$$
\int_{V^{n}} f(\hat{x}) \mathrm{d} \hat{x} \rightarrow \int_{V^{n}} f\left(\mathbf{x}_{\|}, x_{\perp}\right) \mathrm{d} \mathbf{x}_{\|} \mathrm{d} x_{\perp}
$$

whereby

$$
\begin{aligned}
\mathrm{d} \hat{x} & =\mathrm{d} \mathbf{x}^{(0)} \cdots \mathrm{d} \mathbf{x}^{(n)} \text { and } \\
\mathrm{d} x_{\perp} & =\mathrm{d} \mathbf{x}_{\perp}^{(0)} \cdots \mathrm{d} \mathbf{x}_{\perp}^{(n)} \delta\left(\mathbf{x}_{\perp}^{(0)}+\ldots+\mathbf{x}_{\perp}^{(n)}\right) \\
& =\mathrm{d} \mathbf{x}_{\perp}^{(1)} \cdots \mathrm{d} \mathbf{x}_{\perp}^{(n)}
\end{aligned}
$$

We already noted that the mean component of $x_{\perp}$ must be zero, therefore it is sufficient to integrate over only $n$ of the $n+1$ components, or use the $\delta$-function to satisfy this constraint. For the variable transformation (A.3), we now successively 
change the individual variables:

$$
\begin{aligned}
& =\mid \frac{\partial \mathbf{x}_{\|}}{\left.\partial \mathbf{x}^{(0)}\right|_{\mathbf{x}_{\perp}^{(1), \ldots,(n)}}} \begin{array}{l}
\mathrm{d} \mathbf{x}_{\|} \mathrm{d} \mathbf{x}_{\perp}^{(1)} \mathrm{d} \mathbf{x}_{\perp}^{(2)} \cdots \mathrm{d} \mathbf{x}_{\perp}^{(n)} \\
(1) \mathrm{d} \mathbf{x}_{\perp}^{(2)} \cdots \mathrm{d} \mathbf{x}_{\perp}^{(n)}
\end{array} \\
& =\left|\frac{\partial \mathbf{x}_{\|}}{\partial \mathbf{x}^{(0)}}\right|_{\mathbf{x}_{\perp}^{(1), \ldots,(n)}}\left|\frac{\partial \mathbf{x}_{\perp}^{(1)}}{\partial \mathbf{x}^{(1)}}\right|_{\mathbf{x}^{(0)}, \mathbf{x}_{\perp}^{(2), \ldots,(n)}} \mathrm{d} \mathbf{x}^{(0)} \mathrm{d} \mathbf{x}^{(1)} \mathrm{d} \mathbf{x}_{\perp}^{(2)} \cdots \mathrm{d} \mathbf{x}_{\perp}^{(n)} \\
& =\left|\frac{\partial \mathbf{x}_{\|}}{\partial \mathbf{x}^{(0)}}\right|_{\mathbf{x}_{\perp}^{(1), \ldots,(n)}}\left|\frac{\partial \mathbf{x}_{\perp}^{(1)}}{\partial \mathbf{x}^{(1)}}\right|_{\mathbf{x}^{(0)}, \mathbf{x}_{\perp}^{(2), \ldots,(n)}} \ldots\left|\frac{\partial \mathbf{x}_{\perp}^{(n)}}{\partial \mathbf{x}^{(n)}}\right|_{\mathbf{x}^{(0), \ldots,(n-1)}} \mathrm{d} \mathbf{x}^{(0)} \mathrm{d} \mathbf{x}^{(1)} \mathrm{d} \mathbf{x}^{(2)} \cdots \mathrm{d} \mathbf{x}^{(n)}
\end{aligned}
$$

In each transformation the determinant of the Jacobian matrix for (e.g. $\left|\frac{\partial \mathbf{x}_{\perp}^{(n)}}{\partial \mathbf{x}^{(n)}}\right|$ ) has to be evaluated keeping the variables constant, which are currently not transformed (denoted by $|\cdot|_{\mathbf{x}^{(0), \ldots,(n-1)}}$ ). With the definition (A.1) and (A.2) one can easily confirm that

$$
\left|\frac{\partial \mathbf{x}_{\|}}{\partial \mathbf{x}^{(0)}}\right|_{\mathbf{x}_{\perp}^{(1), \ldots,(n)}}=(n+1)^{D / 2}
$$

and for $1 \leq j \leq n$ :

$$
\left|\frac{\partial \mathbf{x}_{\perp}^{(j)}}{\partial \mathbf{x}^{(j)}}\right|_{\mathbf{x}^{(0), \ldots,(j-1), \mathbf{x}_{\perp}^{(j+1), \ldots,(n)}}}=\left(\frac{j}{j+1}\right)^{D}
$$

Therefore we get:

$$
\begin{aligned}
\left|\frac{\partial \mathbf{x}_{\|}}{\partial \mathbf{x}^{(0)}}\right|_{\mathbf{x}_{\perp}^{(1), \ldots,(n)}}\left|\frac{\partial \mathbf{x}_{\perp}^{(1)}}{\partial \mathbf{x}^{(1)}}\right|_{\mathbf{x}^{(0)}, \mathbf{x}_{\perp}^{(2), \ldots,(n)}} \cdots\left|\frac{\partial \mathbf{x}_{\perp}^{(n)}}{\partial \mathbf{x}^{(n)}}\right|_{\mathbf{x}^{(0), \ldots,(n-1)}}= \\
=(n+1)^{D / 2}\left(\frac{1}{2}\right)^{D}\left(\frac{2}{3}\right)^{D} \cdots\left(\frac{n}{n+1}\right)^{D} \\
=(n+1)^{D / 2}\left(\frac{1}{n+1}\right)^{D}=(n+1)^{-D / 2}
\end{aligned}
$$

Hence the transformation can be written as:

$$
\int_{V^{n}} f(\hat{x}) \mathrm{d} \hat{x}=(n+1)^{D / 2} \int_{V^{n}} f\left(\mathbf{x}_{\|}, x_{\perp}\right) \mathrm{d} \mathbf{x}_{\|} \mathrm{d} x_{\perp},
$$


Appendix B

\section{Calculations for the Randomly Cross-Linked Particle Model}

\section{Contents}

B.1 Calculation of the Replica Free Energy . . . . . . . . . 128

B.1.1 Emergence of the Replica Free Energy . . . . . . . . . . . 128

B.1.2 Introduction of the Replicated Density Field . . . . . . . . 129

B.2 Hubbard-Stratonovich Transformation . . . . . . . . 130

B.2.1 Applying the Hubbard-Stratonovich Transformation . . . . . 130

B.2.2 Meaning of the Order Parameter $\Omega(\hat{q}) \ldots \ldots \ldots \ldots$

B.3 Expansion of the replica free energy to 3rd Order in $\Omega \ldots 134$

B.4 Fluctuations around the Saddle-Point Solution $\ldots \ldots \ldots 135$

B.5 Replica Free Energy with Shear Deformations . . . . . . 136

B.5.1 Preparing the Replica Free Energy for the Ansatz . . . . . 136

B.5.2 Expansion of the one-particle partition function $\mathfrak{z} \ldots \ldots . .137$

B.5.3 Insertion of the Order Parameter in $\mathfrak{z} \ldots \ldots . \ldots 137$

B.5.4 Insertion of the Order Parameter in $f_{n+1}\{\Omega\} \ldots \ldots \ldots . \ldots 145$

B.5.5 Recomposing the Replica Free Energy . . . . . . . . . . . . 146

B.5.6 Analysis of the quantity $\Xi_{r, a^{2}} \ldots \ldots \ldots \ldots$

B.6 Auxiliary Calculations for the Results Section . . . . . . 150

B.6.1 Distribution of the Number of Cross-Links . . . . . . . . . . . 150

B.6.2 Shear modulus . . . . . . . . . . . . . . . . . . . 152

B.6.3 Square of the expansion for $(1-Q) \ldots \ldots \ldots \ldots$

B.7 The Order Parameter in the One Replica Sector . . . . . 154 


\section{B.1 Calculation of the Replica Free Energy}

\section{B.1.1 Emergence of the Replica Free Energy}

Here we plug Eq. (2.12) into Eq. (2.11) and define $\tilde{\mu}^{2}:=\frac{\mu^{2}}{2 N \phi}$ to get:

$$
\begin{aligned}
{\left[Z_{\mathcal{C}}^{n}\right]=} & \frac{1}{\mathcal{Z}_{1}} \sum_{M=0}^{\infty} \sum_{\substack{i_{1}, \ldots, i_{M}, j_{1}, \ldots, j_{M}=1}}^{N} \frac{\left(\tilde{\mu}^{2}\right)^{M}}{M !} \\
& \times \int\left(\prod_{j=1}^{N} \mathrm{D} \hat{r}_{j}\right) \exp \left(-\frac{1}{2 a^{2}} \sum_{e=1}^{M}\left(\hat{r}_{i_{e}}-\hat{r}_{j_{e}}\right)^{2}-H_{\mathrm{ev}}^{(n+1)}\right)
\end{aligned}
$$

A part of this expression can be simplified:

$$
\begin{aligned}
& \sum_{M=0}^{\infty} \sum_{\substack{i_{1}, \ldots, i_{M}, j_{1}, \ldots, j_{M}=1}}^{N} \frac{\left(\tilde{\mu}^{2}\right)^{M}}{M !} \exp \left(-\frac{1}{2 a^{2}} \sum_{e=1}^{M}\left(\hat{r}_{i_{e}}-\hat{r}_{j_{e}}\right)^{2}\right) \\
= & \sum_{M=0}^{\infty} \frac{\left(\tilde{\mu}^{2}\right)^{M}}{M !} \sum_{\substack{i_{1}, \ldots, i_{M}, j_{1}, \ldots, j_{M}=1}}^{N} \prod_{e=1}^{M} \exp \left(-\frac{1}{2 a^{2}}\left(\hat{r}_{i_{e}}-\hat{r}_{j_{e}}\right)^{2}\right) \\
= & \sum_{M=0}^{\infty} \frac{\left(\tilde{\mu}^{2}\right)^{M}}{M !}\left(\sum_{i, j=1}^{N} \exp \left(-\frac{\left(\hat{r}_{i}-\hat{r}_{j}\right)^{2}}{2 a^{2}}\right)\right)^{M} \\
= & \exp \left(\tilde{\mu}^{2} \sum_{i, j=1}^{N} \exp \left(-\frac{\left(\hat{r}_{i}-\hat{r}_{j}\right)^{2}}{2 a^{2}}\right)\right)
\end{aligned}
$$

Hence Eq. (B.1) becomes:

$$
\begin{aligned}
{\left[Z^{n}\right] } & =\frac{1}{\mathcal{Z}_{1}} \int\left(\prod_{j=1}^{N} \mathrm{D} \hat{r}_{j}\right) \exp \left(\tilde{\mu}^{2} \sum_{i, j=1}^{N} \exp \left(-\frac{\left(\hat{r}_{i}-\hat{r}_{j}\right)^{2}}{2 a^{2}}\right)-H_{\mathrm{ev}}^{(n+1)}\right) \\
& =: \frac{\mathcal{Z}_{n+1}}{\mathcal{Z}_{1}}
\end{aligned}
$$

where

$$
\begin{aligned}
\mathcal{Z}_{n+1} & =\int\left(\prod_{j=1}^{N} \mathrm{D} \hat{r}_{j}\right) \exp \left(-N \tilde{f}_{n+1}\left\{\hat{r}_{j}\right\}\right) \\
\text { and } \quad \tilde{f}_{n+1}\left\{\hat{r}_{j}\right\} & =-\frac{\tilde{\mu}^{2}}{N} \sum_{i, j=1}^{N} \exp \left(-\frac{\left(\hat{r}_{i}-\hat{r}_{j}\right)^{2}}{2 a^{2}}\right)+\frac{H_{\mathrm{ev}}^{(n+1)}}{N}
\end{aligned}
$$




\section{B.1.2 Introduction of the Replicated Density Field}

In terms of the replicated density field $\mathrm{O}(\hat{x})=\frac{1}{N} \sum_{j=1}^{N} \delta\left(\hat{x}-\hat{r}_{j}\right)$, Eq. (2.16), we can rewrite the cross-linking term of Eq. (2.14c)/(B.4b):

$$
\begin{aligned}
& \sum_{i, j=1}^{N} \exp \left(-\frac{\left(\hat{r}_{i}-\hat{r}_{j}\right)^{2}}{2 a^{2}}\right) \\
= & \sum_{i, j=1}^{N} \int \mathrm{d} \hat{x} \mathrm{~d} \hat{y} \delta\left(\hat{x}-\hat{r}_{i}\right) \delta\left(\hat{y}-\hat{r}_{j}\right) \exp \left(-\frac{(\hat{x}-\hat{y})^{2}}{2 a^{2}}\right) \\
= & N^{2} \int \mathrm{d} \hat{x} \mathrm{~d} \hat{y} \mathrm{O}(\hat{x}) \mathrm{O}(\hat{y}) \exp \left(-\frac{(\hat{x}-\hat{y})^{2}}{2 a^{2}}\right)
\end{aligned}
$$

The Fourier transform of the Gaussian is

$$
\int \mathrm{d} \hat{x} \exp (-\mathrm{i} \hat{q} \hat{x}) \exp \left(-\frac{\hat{x}^{2}}{2 a^{2}}\right)=\left(2 \pi a^{2}\right)^{D(n+1) / 2} \exp \left(-\frac{\hat{q}^{2} a^{2}}{2}\right),
$$

and thus we can use Parseval's identity to transform the whole term (B.5) into Fourier space:

$$
\begin{aligned}
& N^{2} \int \mathrm{d} \hat{x} \mathrm{~d} \hat{y} \mathrm{O}(\hat{x}) \mathrm{O}(\hat{y}) \exp \left(-\frac{(\hat{x}-\hat{y})^{2}}{2 a^{2}}\right) \\
= & N^{2} \frac{\left(2 \pi a^{2}\right)^{D(n+1) / 2}}{V^{n+1}} \sum_{\hat{q}} \mathrm{O}(\hat{q}) \mathrm{O}(-\hat{q}) \exp \left(-\frac{\hat{q}^{2} a^{2}}{2}\right) \\
= & N^{2} \phi^{n+1} \sum_{\hat{q}}|\mathrm{O}(\hat{q})|^{2} \Delta(\hat{q}),
\end{aligned}
$$

with

$$
\Delta(\hat{q}):=\exp \left(-\frac{\hat{q}^{2} a^{2}}{2}\right)
$$

Analogously we can rewrite the excluded volume Hamiltonian in terms of the replicated density field:

$$
\begin{aligned}
H_{\mathrm{ev}}^{(n+1)} & =\frac{\lambda}{2} \sum_{\alpha=0}^{n} \sum_{i, j=1}^{N} U\left(\mathbf{r}_{i}^{(\alpha)}-\mathbf{r}_{j}^{(\alpha)}\right) \\
& =\frac{\lambda}{2} \sum_{\alpha=0}^{n} \sum_{i, j=1}^{N} \int \mathrm{d} \mathbf{x} \mathrm{d} \mathbf{y} \delta\left(\mathbf{x}-\mathbf{r}_{i}^{(\alpha)}\right) \delta\left(\mathbf{y}-\mathbf{r}_{j}^{(\alpha)}\right) U(\mathbf{x}-\mathbf{y}) \\
& =\frac{\lambda N^{2}}{2} \sum_{\alpha=0}^{n} \int \mathrm{d} \mathbf{x} \mathrm{d} \mathbf{y} \mathrm{O}^{(\alpha)}(\mathbf{x}) \mathrm{O}^{(\alpha)}(\mathbf{y}) U(\mathbf{x}-\mathbf{y}) \\
& =\frac{\lambda N^{2}}{2 V} \sum_{\alpha=0}^{n} \sum_{\mathbf{q}}\left|\mathrm{O}^{(\alpha)}(\mathbf{q})\right|^{2} U(\mathbf{q})
\end{aligned}
$$


Now, we can plug Eqs. (B.7) and (B.9) back into Eq. (2.14c) and get:

$$
\tilde{f}_{n+1}\left\{\hat{r}_{j}\right\}=-\phi^{n} \frac{\mu^{2}}{2} \sum_{\hat{q}}|\mathrm{O}(\hat{q})|^{2} \Delta(\hat{q})+\frac{\lambda n_{0}}{2} \sum_{\alpha=0}^{n} \sum_{\mathbf{q}}\left|\mathrm{O}^{(\alpha)}(\mathbf{q})\right|^{2} U(\mathbf{q})
$$

with the mean particle density $n_{0}=N / V$.

The sums in Eq. (B.10) include $\hat{q}=\hat{0}$ and $\mathbf{q}=\mathbf{0}$. Those terms are mere constants, since $\mathrm{O}(\hat{0})=\mathrm{O}^{(\alpha)}(\mathbf{0})=1$. To make that clearer in the main calculation, it is useful to exclude those terms from the sums and rewrite Eq. (B.10) as

$$
\begin{aligned}
\tilde{f}_{n+1}\left\{\hat{r}_{j}\right\}= & \overbrace{-\phi^{n} \frac{\mu^{2}}{2}+(n+1) \frac{\lambda n_{0}}{2}}^{=: f_{0}} \\
& -\phi^{n} \frac{\mu^{2}}{2} \sum_{\hat{q} \neq \hat{0}}|\mathrm{O}(\hat{q})|^{2} \Delta(\hat{q})+\frac{n_{0} \lambda}{2} \sum_{\alpha=0}^{n} \sum_{\mathbf{q} \neq \mathbf{0}}\left|\mathrm{O}^{(\alpha)}(\mathbf{q})\right|^{2} U(\mathbf{q})
\end{aligned}
$$

\section{B.2 Hubbard-Stratonovich Transformation}

\section{B.2.1 Applying the Hubbard-Stratonovich Transformation}

The Hubbard-Stratonovich transformation is used to linearize a quadratic term in an exponential, whereby one has to put up with the integration over a new variable. In its basic form, the Hubbard-Stratonovich transformation is nothing more than a complex Gaussian integral:

$$
\begin{aligned}
\exp \left(c|w|^{2}\right) & =\frac{c}{\pi} \int_{\mathbb{C}} \mathrm{d} z \exp \left(-c|z|^{2}+2 c \operatorname{Re}\left(z w^{*}\right)\right) \\
\exp \left(-c|w|^{2}\right) & =\frac{c}{\pi} \int_{\mathbb{C}} \mathrm{d} z \exp \left(-c|z|^{2}+2 \mathrm{i} c \operatorname{Re}\left(z w^{*}\right)\right) .
\end{aligned}
$$

Here, $c>0$ and real, and $w^{*}$ is the complex conjugate of $w$. Furthermore $\mathrm{d} z=$ $\mathrm{d}(\operatorname{Re} z) \mathrm{d}(\operatorname{Im} z)$, i.e. one has to integrate twice.

Now we want to apply this relation to Eq. (2.24a), with $w=\mathrm{O}(\hat{q})$ successively for all $\hat{q}$. Looking at the definition of the density $\mathrm{O}(\hat{q})$, Eq. $(2.17 \mathrm{a})$, we see that it fulfills the relation $\mathrm{O}(-\hat{q})=\mathrm{O}^{*}(\hat{q})$, and hence $\mathrm{O}(\hat{q})$ and $\mathrm{O}(-\hat{q})$ are not independent variables. In particular $|\mathrm{O}(\hat{q})|^{2}=|\mathrm{O}(-\hat{q})|^{2}$. That means, we automatically transform the $|\mathrm{O}(\hat{q})|^{2}$-term along with the corresponding $|\mathrm{O}(-\hat{q})|^{2}$-term.

To account for that particularity, it is useful to restrict the sums of Eq. (2.24a) to a half-space with $\hat{q} \cdot \hat{e}>0$ with $\hat{e}=(\mathbf{e}, \ldots, \mathbf{e})$ for arbitrary $\mathbf{e} \neq \mathbf{0}$. Thereby we combine the terms for $\hat{q}$ and $-\hat{q}$ in the sum and furthermore use $\Delta(\hat{q})+\Delta(-\hat{q})=2 \Delta(\hat{q})$ and $\tilde{\lambda}(\hat{q})+\tilde{\lambda}(-\hat{q})=2 \tilde{\lambda}(\hat{q})$ (this is true, because all interactions are symmetric). Thus 
we can write the replica partition function $(2.14 \mathrm{c})$, with the free energy (2.24a), as:

$$
\begin{aligned}
& \frac{\mathcal{Z}_{n+1}}{\exp \left(-N f_{0}\right)} \\
& =\int\left(\prod_{j=1}^{N} \mathrm{D} \hat{r}_{j}\right) \exp \left(N \phi^{n} \mu^{2} \sum_{\substack{\hat{q} \in \operatorname{HRS} \\
\hat{q} \cdot \hat{e}>0}}|\mathrm{O}(\hat{q})|^{2} \Delta(\hat{q})-N \sum_{\substack{\hat{q} \in 1 \mathrm{RS} \\
\hat{q} \cdot \hat{e}>0}}|\mathrm{O}(\hat{q})|^{2} \tilde{\lambda}(\hat{q})\right) .
\end{aligned}
$$

Now we apply the Hubbard-Stratonovich transformation (B.12) with $w=\mathrm{O}(\hat{q})$. For any $\hat{q} \in \mathrm{HRS}, \hat{q} \cdot \hat{e}>0$ we use (B.12a) and set $c=N \phi^{n} \mu^{2} \Delta(\hat{q})$ :

$$
\begin{aligned}
& \exp \left(N \phi^{n} \mu^{2}|\mathrm{O}(\hat{q})|^{2} \Delta(\hat{q})\right) \\
= & \int \frac{N \phi^{n} \mu^{2} \Delta(\hat{q}) \mathrm{d} \Omega(\hat{q})}{\pi} \exp \left(-N \phi^{n} \mu^{2} \Delta(\hat{q})|\Omega(\hat{q})|^{2}+2 N \phi^{n} \mu^{2} \Delta(\hat{q}) \operatorname{Re}(\Omega(\hat{q}) \mathrm{O}(-\hat{q}))\right)
\end{aligned}
$$

and for $\hat{q} \in 1 \mathrm{RS}, \hat{q} \cdot \hat{e}>0$, we use (B.12b) with $c=N \tilde{\lambda}(\hat{q})$ :

$$
\begin{aligned}
& \exp \left(-N|\mathrm{O}(\hat{q})|^{2} \tilde{\lambda}(\hat{q})\right) \\
& \quad=\int \frac{N \tilde{\lambda}(\hat{q}) \mathrm{d} \Omega(\hat{q})}{\pi} \exp \left(-N|\Omega(\hat{q})|^{2} \tilde{\lambda}(\hat{q})+2 \mathrm{i} N \tilde{\lambda}(\hat{q}) \operatorname{Re}(\Omega(\hat{q}) \mathrm{O}(-\hat{q}))\right)
\end{aligned}
$$

With those two relations, Eq. (B.13) becomes:

$$
\begin{aligned}
& \frac{\mathcal{Z}_{n+1}}{\exp \left(-N f_{0}\right)}= \int \mathcal{D} \Omega \exp \left(-N \phi^{n} \mu^{2} \sum_{\substack{\hat{q} \in \mathrm{HRS} \\
\hat{q} \cdot \hat{e}>0}}|\Omega(\hat{q})|^{2} \Delta(\hat{q})-N \sum_{\substack{\hat{q} \in 1 \mathrm{RS} \\
\hat{q} \cdot \hat{e}>0}}|\Omega(\hat{q})|^{2} \tilde{\lambda}(\hat{q})\right) \\
& \times \int\left(\prod_{j=1}^{N} \mathrm{D} \hat{r}_{j}\right) \exp \left(2 N \phi^{n} \mu^{2} \sum_{\substack{\hat{q} \in \mathrm{HRS} \\
\hat{q} \cdot \hat{e}>0}} \Delta(\hat{q}) \operatorname{Re}(\Omega(\hat{q}) \mathrm{O}(-\hat{q}))\right. \\
&\left.-2 \mathrm{i} N \sum_{\substack{\hat{q} \in 1 \mathrm{RS} \\
\hat{q} \cdot \hat{e}>0}} \tilde{\lambda}(\hat{q}) \operatorname{Re}(\Omega(\hat{q}) \mathrm{O}(-\hat{q}))\right),
\end{aligned}
$$

where we introduced the measure

$$
\mathcal{D} \Omega=\prod_{\substack{\hat{q} \in 1 \mathrm{RS} \\ \hat{q} \cdot \hat{e}>0}} \frac{N \tilde{\lambda}(\hat{q}) \mathrm{d} \Omega(\hat{q})}{\pi} \cdot \prod_{\substack{\hat{q} \in \operatorname{HRS} \\ \hat{q} \cdot \hat{e}>0}} \frac{N \phi^{n} \mu^{2} \Delta(\hat{q}) \mathrm{d} \Omega(\hat{q})}{\pi} .
$$

Now we abolish the constraint $\hat{q} \cdot \hat{e}>0$ in the sums. To this end, we define the field $\Omega(\hat{q})$ also for $\hat{q} \cdot \hat{e}<0$ by

$$
\Omega(-\hat{q})=\Omega^{*}(\hat{q})
$$


We also plug in the definition of $\mathrm{O}(\hat{q})$, Eq. (2.17a), and get:

$$
\begin{aligned}
& \frac{\mathcal{Z}_{n+1}}{\exp \left(-N f_{0}\right)}=\int \mathcal{D} \Omega \exp \left(-N \frac{\phi^{n} \mu^{2}}{2} \sum_{\hat{q} \in \mathrm{HRS}}|\Omega(\hat{q})|^{2} \Delta(\hat{q})-\frac{N}{2} \sum_{\hat{q} \in \mathrm{RS}}|\Omega(\hat{q})|^{2} \tilde{\lambda}(\hat{q})\right) \\
& \times \int\left(\prod_{j=1}^{N} \mathrm{D} \hat{r}_{j}\right) \exp \left(\phi^{n} \mu^{2} \sum_{j=1}^{N} \sum_{\hat{q} \in \mathrm{HRS}} \Delta(\hat{q}) \Omega(\hat{q}) \mathrm{e}^{-\mathrm{i} \hat{\mathrm{q}} \hat{r}_{j}}+\mathrm{i} \sum_{j=1}^{N} \sum_{\hat{q} \in \mathrm{RSS}} \tilde{\lambda}(\hat{q}) \Omega(\hat{q}) \mathrm{e}^{-\mathrm{i} \hat{q} \hat{r}_{j}}\right)
\end{aligned}
$$

At this point, we can see that the Hubbard-Stratonovich transformation pays off: Now, the density $\sum_{j=1}^{N} \mathrm{e}^{-\mathrm{i} \hat{q} \hat{r}_{j}}$ appears linearly in the exponential and we can rewrite the difficult integral $\int\left(\prod_{j=1}^{N} \mathrm{D} \hat{r}_{j}\right)$ in the last line of Eq. (B.18) in the following way:

$$
\begin{aligned}
\frac{\mathcal{Z}_{n+1}}{\exp \left(-N f_{0}\right)} & =\int \mathcal{D} \Omega \exp \left(-N \frac{\phi^{n} \mu^{2}}{2} \sum_{\hat{q} \in \mathrm{HRS}} \Delta(\hat{q})|\Omega(\hat{q})|^{2}-\frac{N}{2} \sum_{\hat{q} \in 1 \mathrm{RS}} \tilde{\lambda}(\hat{q})|\Omega(\hat{q})|^{2}\right) \\
& \times(\underbrace{\int \mathrm{D} \hat{r} \exp \left(\phi^{n} \mu^{2} \sum_{\hat{q} \in \mathrm{HRS}} \Delta(\hat{q}) \Omega(\hat{q}) \mathrm{e}^{-\mathrm{i} \hat{q} \hat{r}}+\mathrm{i} \sum_{\hat{q} \in 1 \mathrm{RS}} \tilde{\lambda}(\hat{q}) \Omega(\hat{q}) \mathrm{e}^{-\mathrm{i} \hat{q} \hat{r}}\right)}_{=: \mathfrak{z}})^{N} .
\end{aligned}
$$

And this form can be written in a very descriptive way as:

$$
\mathcal{Z}_{n+1}=\exp \left(-N f_{0}\right) \int \mathcal{D} \Omega \exp \left(-N f_{n+1}\{\Omega\}\right)
$$

with the replica free energy

$$
f_{n+1}\{\Omega\}=\frac{\phi^{n} \mu^{2}}{2} \sum_{\hat{q} \in \mathrm{HRS}} \Delta(\hat{q})|\Omega(\hat{q})|^{2}+\frac{1}{2} \sum_{\hat{q} \in 1 \mathrm{RS}} \tilde{\lambda}(\hat{q})|\Omega(\hat{q})|^{2}-\frac{1}{\not{X}} \ln \mathfrak{z}^{\not \mathcal{}}
$$

and an effective one-particle partition function

$$
\mathfrak{z}=\int \mathrm{D} \hat{r} \exp \left(\phi^{n} \mu^{2} \sum_{\hat{q} \in \mathrm{HRS}} \Delta(\hat{q}) \Omega(\hat{q}) \mathrm{e}^{-\mathrm{i} \hat{q} \hat{r}}+\mathrm{i} \sum_{\hat{q} \in 1 \mathrm{RS}} \tilde{\lambda}(\hat{q}) \Omega(\hat{q}) \mathrm{e}^{-\mathrm{i} \hat{q} \hat{r}}\right)
$$

\section{B.2.2 Meaning of the Order Parameter $\Omega(\hat{q})$}

In the basic form of the Hubbard-Stratonovich transformation with plus sign, Eq. (B.12a), one can calculate how the two variables $w$ and $z$ are connected. To see 
this, we have a look at the expectation value:

$$
\begin{aligned}
\left\langle|w-z|^{2}\right\rangle_{+} & :=\frac{\int \mathrm{d} z|w-z|^{2} \exp \left(-c|z|^{2}+2 c \operatorname{Re}\left(z w^{*}\right)\right)}{\int \mathrm{d} z \exp \left(-c|z|^{2}+2 c \operatorname{Re}\left(z w^{*}\right)\right)} \\
& =\frac{\int \mathrm{d} z|w-z|^{2} \exp \left(-c|z|^{2}+2 c \operatorname{Re}\left(z w^{*}\right)\right)}{\frac{\pi}{c} \exp \left(c|w|^{2}\right)} \\
& =\frac{c}{\pi} \int \mathrm{d} z|w-z|^{2} \exp \left(-c|w-z|^{2}\right)
\end{aligned}
$$

where we used $|w-z|^{2}=|z|^{2}+|w|^{2}-2 \operatorname{Re}\left(z w^{*}\right)$. Then:

$$
\begin{aligned}
\left\langle|w-z|^{2}\right\rangle_{+} & =\frac{c}{\pi}\left(-\frac{\partial}{\partial c}\right) \int \mathrm{d} z \exp \left(-c|w-z|^{2}\right)=-\frac{c}{\pi} \frac{\partial}{\partial c} \frac{\pi}{c} \\
& =\frac{1}{c}
\end{aligned}
$$

Using this relation for the transformation (B.14a), we get with $c \propto N$ :

$$
\left\langle|\mathrm{O}(\hat{q})-\Omega(\hat{q})|^{2}\right\rangle_{\Omega(\hat{q})} \propto \frac{1}{N} \text { for all } \hat{q} \in \mathrm{HRS},
$$

for an arbitrary particle configuration $\left\{\hat{r}_{j}\right\}$. The angled brackets $\langle\cdot\rangle_{\Omega(\hat{q})}$ mean averaging over all $\Omega(\hat{q})$ with the statistical weight as in Eq. (B.14a). If we additionally average Eq. (B.23) over all particle configurations $\left\{\hat{r}_{j}\right\}$, in analogy to the step from Eq. (B.14) to Eq. (B.15), we immediately get:

$$
\left\langle|\mathrm{O}(\hat{q})-\Omega(\hat{q})|^{2}\right\rangle_{f_{n+1}} \propto \frac{1}{N} \quad \text { for all } \quad \hat{q} \in \mathrm{HRS}
$$

where $\langle\cdot\rangle_{f_{n+1}}$ is now averaging with respect to the replica free energy $f_{n+1}\{\Omega\}$ (before reducing the integral $\int \mathrm{d} \hat{r}_{1} \cdots \mathrm{d} \hat{r}_{N}$ to an integral over one particle). This result is very convenient: It means that the difference between $\mathrm{O}(\hat{q})$ and $\Omega(\hat{q})$ vanishes in the thermodynamic limit and hence, both can studied equivalently.

For $\hat{q} \in 1$ RS we used the Hubbard-Stratonovich transformation with minus sign, Eq. (B.12b). For that case one can show an analogous relation:

$$
\left\langle|\mathrm{i} w-z|^{2}\right\rangle_{-}:=\frac{\int \mathrm{d} z|\mathrm{i} w-z|^{2} \exp \left(-c|z|^{2}+2 \mathrm{i} c \operatorname{Re}\left(z w^{*}\right)\right)}{\int \mathrm{d} z \exp \left(-c|z|^{2}+2 \mathrm{i} c \operatorname{Re}\left(z w^{*}\right)\right)}=\frac{1}{c} .
$$

We apply this relation to the transformation (B.14b) and get:

$$
\left\langle|\mathrm{iO}(\hat{q})-\Omega(\hat{q})|^{2}\right\rangle_{f_{n+1}} \propto \frac{1}{N} \quad \text { for all } \quad \hat{q} \in 1 \mathrm{RS},
$$

which means that in the $1 \mathrm{RS}, \Omega(\hat{q})$ and $\mathrm{iO}(\hat{q})$ are the same in the thermodynamic limit. 


\section{B.3 Expansion of the replica free energy to 3rd Order in $\Omega$}

Here, we want to expand the replica free energy, Eq. (2.28), to the lowest necessary order, which is $\mathcal{O}\left(\Omega^{3}\right)$. As one can easily see, the expansion of $\mathfrak{z}$, Eq. (2.28b), yields

$$
\begin{aligned}
\mathfrak{z}=\int \mathrm{D} \hat{r}(1 & +\phi^{n} \mu^{2} \sum_{\hat{q} \in \mathrm{HRS}} \Delta(\hat{q}) \Omega(\hat{q}) \mathrm{e}^{-\mathrm{i} \hat{q} \hat{r}} \\
& +\frac{1}{2 !} \phi^{2 n} \mu^{4} \sum_{\hat{q}_{1}, \hat{q}_{2} \in \mathrm{HRS}} \Delta\left(\hat{q}_{1}\right) \Delta\left(\hat{q}_{2}\right) \Omega\left(\hat{q}_{1}\right) \Omega\left(\hat{q}_{2}\right) \mathrm{e}^{-\mathrm{i}\left(\hat{q}_{1}+\hat{q}_{2}\right) \hat{r}} \\
& \left.+\frac{1}{3 !} \phi^{3 n} \mu^{6} \sum_{\hat{q}_{1}, \hat{q}_{2}, \hat{q}_{3} \in \mathrm{HRS}} \Delta\left(\hat{q}_{1}\right) \Delta\left(\hat{q}_{2}\right) \Delta\left(\hat{q}_{3}\right) \Omega\left(\hat{q}_{1}\right) \Omega\left(\hat{q}_{2}\right) \Omega\left(\hat{q}_{3}\right) \mathrm{e}^{-\mathrm{i}\left(\hat{q}_{1}+\hat{q}_{2}+\hat{q}_{3}\right) \hat{r}}\right) \\
= & +\frac{\phi^{2 n} \mu^{4}}{2 !} \sum_{\hat{q}_{1}, \hat{q}_{2} \in \mathrm{HRS}} \Delta\left(\hat{q}_{1}\right) \Delta\left(\hat{q}_{2}\right) \Omega\left(\hat{q}_{1}\right) \Omega\left(\hat{q}_{2}\right) \delta_{\hat{q}_{1}+\hat{q}_{2}, \hat{0}} \\
& +\frac{\phi^{3 n} \mu^{6}}{3 !} \sum_{\hat{q}_{1}, \hat{q}_{2}, \hat{q}_{3} \in \mathrm{HRS}} \Delta\left(\hat{q}_{1}\right) \Delta\left(\hat{q}_{2}\right) \Delta\left(\hat{q}_{3}\right) \Omega\left(\hat{q}_{1}\right) \Omega\left(\hat{q}_{2}\right) \Omega\left(\hat{q}_{3}\right) \delta_{\hat{q}_{1}+\hat{q}_{2}+\hat{q}_{3}, \hat{0}}+\mathcal{O}\left(\Omega^{4}\right),
\end{aligned}
$$

and with the expansion $\Delta(\hat{q})=\exp \left(-a^{2} \hat{q}^{2} / 2\right)=1-a^{2} \hat{q}^{2} / 2+\mathcal{O}\left(\hat{q}^{4}\right)$, we get:

$$
\begin{aligned}
\ln \mathfrak{z}= & \frac{\phi^{2 n} \mu^{4}}{2} \sum_{\hat{q} \in \mathrm{HRS}}\left(1-\frac{2 a^{2} \hat{q}^{2}}{2}\right) \Omega(\hat{q}) \Omega(-\hat{q}) \\
& +\frac{\phi^{3 n} \mu^{6}}{6} \sum_{\hat{q}_{1}, \hat{q}_{2}, \hat{q}_{3} \in \mathrm{HRS}} \Omega\left(\hat{q}_{1}\right) \Omega\left(\hat{q}_{2}\right) \Omega\left(\hat{q}_{3}\right) \delta_{\hat{q}_{1}+\hat{q}_{2}+\hat{q}_{3}, \hat{0}}+\mathcal{O}\left(\Omega^{4}, \Omega^{3} \hat{q}^{2}, \Omega^{2} \hat{q}^{4}\right) .
\end{aligned}
$$

With Eq. (2.28) the complete replica free energy becomes:

$$
\begin{aligned}
f_{n+1}\{\Omega\}= & \frac{\phi^{n} \mu^{2}}{2} \sum_{\hat{q} \in \mathrm{HRS}}\left(1-\frac{a^{2} \hat{q}^{2}}{2}\right)|\Omega(\hat{q})|^{2}-\frac{\phi^{2 n} \mu^{4}}{2} \sum_{\hat{q} \in \mathrm{HRS}}\left(1-a^{2} \hat{q}^{2}\right) \Omega(\hat{q}) \Omega(-\hat{q}) \\
& -\frac{\phi^{3 n} \mu^{6}}{6} \sum_{\hat{q}_{1}, \hat{q}_{2}, \hat{q}_{3} \in \mathrm{HRS}} \Omega\left(\hat{q}_{1}\right) \Omega\left(\hat{q}_{2}\right) \Omega\left(\hat{q}_{3}\right) \delta_{\hat{q}_{1}+\hat{q}_{2}+\hat{q}_{3}, \hat{0}}+\mathcal{O}\left(\Omega^{4}, \Omega^{3} \hat{q}^{2}, \Omega^{2} \hat{q}^{4}\right)
\end{aligned}
$$

and in the limit $n \rightarrow 0$ :

$$
\begin{aligned}
f_{n+1}\{\Omega\}= & \frac{1}{2} \sum_{\hat{q} \in \mathrm{HRS}}\left(\mu^{2}-\mu^{4}+\left(\mu^{4}-\frac{\mu^{2}}{2}\right) a^{2} \hat{q}^{2}\right)|\Omega(\hat{q})|^{2} \\
& -\mu^{6} \sum_{\hat{q}_{1}, \hat{q}_{2}, \hat{q}_{3} \in \mathrm{HRS}} \Omega\left(\hat{q}_{1}\right) \Omega\left(\hat{q}_{2}\right) \Omega\left(\hat{q}_{3}\right) \delta_{\hat{q}_{1}+\hat{q}_{2}+\hat{q}_{3}, \hat{0}}+\mathcal{O}\left(\Omega^{4}, \Omega^{3} \hat{q}^{2}, \Omega^{2} \hat{q}^{4}\right),
\end{aligned}
$$


or

$$
\begin{aligned}
f_{n+1}\{\Omega\} & =\sum_{\hat{q} \in \mathrm{HRS}}\left(-A \varepsilon+\frac{B}{2} \hat{q}^{2}\right)|\Omega(\hat{q})|^{2} \\
& -C \sum_{\hat{q}_{1}, \hat{q}_{2}, \hat{q}_{3} \in \mathrm{HRS}} \Omega\left(\hat{q}_{1}\right) \Omega\left(\hat{q}_{2}\right) \Omega\left(\hat{q}_{3}\right) \delta_{\hat{q}_{1}+\hat{q}_{2}+\hat{q}_{3}, \hat{0}}+\mathcal{O}\left(\Omega^{4}, \Omega^{3} \hat{q}^{2}, \Omega^{2} \hat{q}^{4}\right)
\end{aligned}
$$

with

$$
\varepsilon=\mu^{2}-1, \quad A=\frac{\mu^{2}}{2}, \quad B=\left(\mu^{4}-\frac{\mu^{2}}{2}\right) a^{2}, \quad C=\frac{\mu^{6}}{6} .
$$

A discussion of the universal free energy, Eq. (B.31), is found in Sec. 2.4.5.

\section{B.4 Fluctuations around the Saddle-Point Solution}

In Sec. 2.6.2, we derived an Ansatz for the order parameter, Eqs. (2.45):

$$
\bar{\Omega}_{u}(\hat{q})=(1-Q) \delta_{\hat{q}, \hat{0}}+Q \omega(\hat{q}) \int \operatorname{Dx} \mathbf{x}_{\|} \exp \left(\mathbf{i} \mathbf{q}_{\|} \mathbf{x}_{\|}+\mathrm{i} q_{\perp} u_{\perp}\left(\mathbf{x}_{\|}\right)\right)
$$

Let us have a look at the integral over $\mathbf{x}_{\|}$of the gel part (marked in blue). Without deformations, $u_{\perp}\left(\mathbf{x}_{\|}\right) \equiv \hat{0}$, it can be performed and yields a $\delta$-function. For this case, the Ansatz reproduces the saddle point solution:

$$
\bar{\Omega}_{u \equiv 0}(\hat{q})=(1-Q) \delta_{\hat{q}, \hat{0}}+Q \omega(\hat{q}) \delta_{\mathbf{q}_{\|}, \mathbf{0}}=\bar{\Omega}_{\mathrm{sp}}(\hat{q})
$$

where $\bar{\Omega}_{\mathrm{sp}}(\hat{q})$ is the Fourier representation of Eq. (2.33).

In the limit of small deformations $u_{\perp}$ and long wavelengths, i.e. small $\hat{q}$, we can expand the exponential to the lowest order in $q_{\perp} u_{\perp}$ :

$$
\begin{aligned}
& \int \mathrm{D} \mathbf{x}_{\|} \exp \left(\mathbf{i} \mathbf{q}_{\|} \mathbf{x}_{\|}+\mathrm{i} q_{\perp} u_{\perp}\left(\mathbf{x}_{\|}\right)\right) \\
= & \int \mathrm{D} \mathbf{x}_{\|} \exp \left(\mathbf{i} \mathbf{q}_{\|} \mathbf{x}_{\|}\right)\left(1+\mathrm{i} q_{\perp} u_{\perp}\left(\mathbf{x}_{\|}\right)\right) \\
= & \delta_{\mathbf{q}_{\|}, 0}+\mathrm{i} q_{\perp} u_{\perp}\left(\mathbf{q}_{\|}\right),
\end{aligned}
$$

with the Fourier transform $u_{\perp}\left(\mathbf{q}_{\|}\right)$of $u_{\perp}\left(\mathbf{x}_{\|}\right)$. That means, we can write the Ansatz (B.33) as:

$$
\begin{aligned}
\bar{\Omega}_{u}(\hat{q}) & =\bar{\Omega}_{\mathrm{sp}}(\hat{q})+\delta \Omega_{u}(\hat{q}) \\
\text { with } \quad \delta \Omega_{u}(\hat{q}) & =\mathcal{O}\left(q_{\perp} u_{\perp}\right),
\end{aligned}
$$

as one can see by comparing Eqs. (B.34) and (B.33), with (B.35) inserted. 


\section{B.5 Replica Free Energy with Shear Deformations}

In this section we will insert the Ansatz (2.45)

$$
\begin{array}{r}
\bar{\Omega}_{u}(\hat{q})=(1-Q) \delta_{\hat{q}, \hat{0}}+Q \omega(\hat{q}) \int \operatorname{Dx} \mathbf{x}_{\|} \exp \left(\mathrm{iq}_{\|} \mathbf{x}_{\|}+\mathrm{i} q_{\perp} u_{\perp}\left(\mathbf{x}_{\|}\right)\right) \\
\text {with } \quad \omega(\hat{q})=\int_{0}^{\infty} \mathrm{d} \xi^{2} \mathcal{P}\left(\xi^{2}\right) \exp \left(-\frac{\hat{q}^{2} \xi^{2}}{2}\right)
\end{array}
$$

into the general expression (2.28) for the replica free energy. We will only consider small, long-wavelength deformations and keep $u_{\perp}$ only to the lowest order, $\left(\partial u_{\perp} / \partial \mathbf{x}_{\|}\right)^{2}$.

\section{B.5.1 Preparing the Replica Free Energy for the Ansatz}

The derived Ansatz for the order parameter (B.37) suggests to define a slightly altered version of the order parameter

$$
\Omega^{\prime}(\hat{q}):=\Omega(\hat{q})-(1-Q) \delta_{\hat{q}, \hat{0}}
$$

so that the Ansatz for this version of the order parameter,

$$
\bar{\Omega}_{u}^{\prime}(\hat{q})=Q \omega(\hat{q}) \int \mathrm{D} \mathbf{x}_{\|} \exp \left(\mathbf{i} \mathbf{q}_{\|} \mathbf{x}_{\|}+\mathrm{i} q_{\perp} u_{\perp}\left(\mathbf{x}_{\|}\right)\right)
$$

does not involve a $\delta$-function and hence becomes easier to handle when summing over $\hat{q}$.

When inserting the Ansatz $\bar{\Omega}_{u}$ into the general replica free energy $f_{n+1}\{\Omega\}$, Eq. (2.28), we note that $f_{n+1}\{\Omega\}$ only involves sums over the higher replica sector (which do not include $\hat{q}=\hat{0}$ ), so we can just $\operatorname{swap} \bar{\Omega}_{u}(\hat{q})$ with $\bar{\Omega}_{u}^{\prime}(\hat{q})$ :

$$
\begin{array}{r}
f_{n+1}\left\{\bar{\Omega}_{u}\right\}=\frac{\phi^{n} \mu^{2}}{2} \sum_{\hat{q} \in \mathrm{HRS}} \Delta(\hat{q})\left|\bar{\Omega}_{u}^{\prime}(\hat{q})\right|^{2}-\ln \mathfrak{z}, \\
\text { with } \mathfrak{z}=\int \mathrm{D} \hat{r} \exp \left(\phi^{n} \mu^{2} \sum_{\hat{q} \in \mathrm{HRS}} \Delta(\hat{q}) \bar{\Omega}_{u}^{\prime}(\hat{q}) \mathrm{e}^{-\mathrm{i} \hat{q} \hat{r}}\right)
\end{array}
$$

To simplify further calculations, we include the 1RS in the sums. We do not need correction terms, since volume conserving deformations keep a constant density and yield $\bar{\Omega}_{u}^{\prime}(\hat{q})=0$ for $\hat{q} \in 1 \mathrm{RS}$, as we show in Appendix B.7. Furthermore, we also include $\hat{q}=\hat{0}$ in the sums and subtract the generated terms (involving $\bar{\Omega}_{u}^{\prime}(\hat{0})=Q$ ) again. With that we can omit the unpleasant constraint $\hat{q} \in$ HRS and sum over 
all $\hat{q}$ :

$$
\begin{aligned}
& f_{n+1}\left\{\bar{\Omega}_{u}\right\}=-\frac{\phi^{n} \mu^{2}}{2} Q^{2}+\frac{\phi^{n} \mu^{2}}{2} \sum_{\hat{q}} \Delta(\hat{q})\left|\bar{\Omega}_{u}^{\prime}(\hat{q})\right|^{2}-\ln \mathfrak{z} \\
& \mathfrak{z}=\exp \left(-\phi^{n} \mu^{2} Q\right) \int \mathrm{D} \hat{r} \exp \left(\phi^{n} \mu^{2} \sum_{\hat{q}} \Delta(\hat{q}) \bar{\Omega}_{u}^{\prime}(\hat{q}) \mathrm{e}^{-\mathrm{i} \hat{q} \hat{r}}\right)
\end{aligned}
$$

\section{B.5.2 Expansion of the one-particle partition function $\mathfrak{z}$}

Now we expand the exponential in the integral of the one-particle partition function $\mathfrak{z}:$

$$
\begin{aligned}
\frac{\mathfrak{z}}{\exp \left(-\phi^{n} \mu^{2} Q\right)} & =\int \mathrm{D} \hat{r} \exp \left(\phi^{n} \mu^{2} \sum_{\hat{q}} \Delta(\hat{q}) \bar{\Omega}_{u}^{\prime}(\hat{q}) \mathrm{e}^{-\mathrm{i} \hat{q} \hat{r}}\right) \\
& =\int \mathrm{D} \hat{r} \sum_{r=0}^{\infty} \frac{1}{r !}\left\{\phi^{n} \mu^{2} \sum_{\hat{q}} \Delta(\hat{q}) \bar{\Omega}_{u}^{\prime}(\hat{q}) \mathrm{e}^{-\mathrm{i} \hat{q} \hat{r}}\right\}^{r} \\
& =\sum_{r=0}^{\infty} \frac{\left(\phi^{n} \mu^{2}\right)^{r}}{r !} \int \mathrm{D} \hat{r}\left\{\sum_{\hat{q}} \Delta(\hat{q}) \bar{\Omega}_{u}^{\prime}(\hat{q}) \mathrm{e}^{-\mathrm{i} \hat{q} \hat{r}}\right\}^{r} \\
& =\sum_{r=0}^{\infty} \frac{\left(\phi^{n} \mu^{2}\right)^{r}}{r !} A_{r}
\end{aligned}
$$

with

$$
A_{r}:=\int_{V^{n+1}} \mathrm{D} \hat{r}\left\{\sum_{\hat{q}} \Delta(\hat{q}) \bar{\Omega}_{u}^{\prime}(\hat{q}) \mathrm{e}^{-\mathrm{i} \hat{q} \hat{r}}\right\}^{r} .
$$

which we now try to calculate.

\section{B.5.3 Insertion of the Order Parameter in $\mathfrak{z}$}

The term $A_{0}$ is very easy:

$$
A_{0}=\int_{V^{n+1}} \mathrm{D} \hat{r}\{\cdots\}^{0}=1
$$

For $A_{1}$ we note that $\int \mathrm{D} \hat{r} \mathrm{e}^{-\mathrm{i} \hat{q} \hat{r}}=\delta_{\hat{q}, \hat{0}}$ and thus:

$$
A_{1}=\int \mathrm{D} \hat{r} \sum_{\hat{q}} \Delta(\hat{q}) \bar{\Omega}_{u}^{\prime}(\hat{q}) \mathrm{e}^{-\mathrm{i} \hat{q} \hat{r}}=\Delta(\hat{0}) \bar{\Omega}_{u}^{\prime}(\hat{0})=Q
$$

Now, let us consider the "difficult" case $r \geq 2$ and plug in the Ansatz (B.39):

$$
\begin{aligned}
A_{r}= & \frac{Q^{r}}{V^{r}(n+1)^{D r / 2}} \int \mathrm{D} \hat{r}\left\{\sum_{\hat{q}} \int \mathrm{d} \mathbf{x}_{\|} \int_{0}^{\infty} \mathrm{d} \xi^{2} \mathcal{P}\left(\xi^{2}\right)\right. \\
& \left.\quad \exp \left(-\frac{a^{2} \hat{q}^{2}}{2}\right) \exp \left(-\frac{\xi^{2} \hat{q}^{2}}{2}\right) \exp \left(\mathrm{i}\left(\mathbf{q}_{\|} \mathbf{x}_{\|}+q_{\perp} u_{\perp}\left(\mathbf{x}_{\|}\right)\right)-\mathrm{i} \hat{q} \hat{r}\right)\right\}^{r}
\end{aligned}
$$


Let us have a closer look at the sum $\sum_{\hat{q}}$ in the first line: It runs over elements $\hat{q}=\frac{2 \pi}{L} \cdot \hat{n}$ with $\hat{n} \in \mathbb{Z}^{D(n+1)}$ and $L=V^{1 / D}$ being the linear dimension of the system. In the thermodynamic limit $L \rightarrow \infty$ the discrete values of this sum move closer and closer and the sum approaches a Riemann integral

$$
\sum_{\hat{q}}(\ldots) \longrightarrow\left(\frac{L}{2 \pi}\right)^{D(n+1)} \int \mathrm{d} \hat{q}(\ldots)
$$

To simplify the notation we also define a $D(n+1)$-dimensional hatted function $\hat{v}\left(\mathbf{x}_{\|}\right)$ such that

$$
\mathbf{v}_{\|}\left(\mathbf{x}_{\|}\right)=\mathbf{x}_{\|} \quad \text { and } \quad v_{\perp}\left(\mathbf{x}_{\|}\right)=u_{\perp}\left(\mathbf{x}_{\|}\right)
$$

so that

$$
\mathbf{q}_{\|} \mathbf{x}_{\|}+q_{\perp} u_{\perp}\left(\mathbf{x}_{\|}\right)=\hat{q} \hat{v}\left(\mathbf{x}_{\|}\right)
$$

and with that we get:

$$
\begin{aligned}
A_{r}= & \frac{Q^{r}}{V^{r}(n+1)^{D r / 2}} \frac{V^{r(n+1)}}{(2 \pi)^{D r(n+1)}} \int \mathrm{D} \hat{r} \\
& \left\{\int \mathrm{d} \hat{q} \int \mathrm{d} \mathbf{x}_{\|} \int_{0}^{\infty} \mathrm{d} \xi^{2} \mathcal{P}\left(\xi^{2}\right) \exp \left(-\frac{\left(a^{2}+\xi^{2}\right) \hat{q}^{2}}{2}\right) \exp \left(\mathrm{i} \hat{q} \hat{v}\left(\mathbf{x}_{\|}\right)-\mathrm{i} \hat{q} \hat{r}\right)\right\}^{r}
\end{aligned}
$$

The integral over $\hat{q}$ is a simple Gaussian integral, which we perform:

$$
\begin{aligned}
A_{r} /\left(\frac{Q^{r} V^{r n}}{(2 \pi)^{D r(n+1)}(n+1)^{D r / 2}}\right)= \\
\quad=\int \mathrm{D} \hat{r}\left\{\int \mathrm{d} \mathbf{x}_{\|} \int_{0}^{\infty} \mathrm{d} \xi^{2} \mathcal{P}\left(\xi^{2}\right)\left(\frac{2 \pi}{a^{2}+\xi^{2}}\right)^{D(n+1) / 2} \exp \left(-\frac{\left(\hat{v}\left(\mathbf{x}_{\|}\right)-\hat{r}\right)^{2}}{2\left(a^{2}+\xi^{2}\right)}\right)\right\}^{r}
\end{aligned}
$$

Now we expand the $r$-th power:

$$
\begin{aligned}
A_{r} /\left(\frac{Q^{r} V^{r n}}{(2 \pi)^{D r(n+1)}(n+1)^{D r / 2}}\right)= \\
=\int_{0}^{\infty} \mathrm{d} \xi_{1}^{2} \mathcal{P}\left(\xi_{1}^{2}\right) \cdots \mathrm{d} \xi_{r}^{2} \mathcal{P}\left(\xi_{r}^{2}\right) \int_{V} \mathrm{D} \hat{r} \int \mathrm{d} \mathbf{x}_{\| 1} \cdots \mathrm{d} \mathbf{x}_{\| r} \\
\prod_{\gamma=1}^{r}\left\{\left(\frac{2 \pi}{a^{2}+\xi_{\gamma}^{2}}\right)^{D(n+1) / 2} \exp \left(-\frac{\left(\hat{v}\left(\mathbf{x}_{\| \gamma}\right)-\hat{r}\right)^{2}}{2\left(a^{2}+\xi_{\gamma}^{2}\right)}\right)\right\}
\end{aligned}
$$

To simplify the notation we furthermore define

$$
\begin{gathered}
\int_{\xi_{1}^{2}, \ldots, \xi_{r}^{2}}:=\int_{0}^{\infty} \mathrm{d} \xi_{1}^{2} \mathcal{P}\left(\xi_{1}^{2}\right) \cdots \mathrm{d} \xi_{r}^{2} \mathcal{P}\left(\xi_{r}^{2}\right) \\
C_{\gamma}:=\frac{1}{a^{2}+\xi_{\gamma}^{2}} \quad \text { and } \quad C:=\sum_{\gamma=1}^{r} C_{\gamma}
\end{gathered}
$$


With that, Eq. (B.52) becomes:

$$
\begin{array}{r}
A_{r} /\left(\frac{Q^{r} V^{r n}}{(2 \pi)^{D r(n+1)}(n+1)^{D r / 2}}\right)=\int_{\xi_{1}^{2}, \ldots, \xi_{r}^{2}}\left(\prod_{\gamma=1}^{r}\left(2 \pi C_{\gamma}\right)^{D(n+1) / 2}\right) \\
\times \int \mathrm{d} \mathbf{x}_{\| 1} \cdots \mathrm{d} \mathbf{x}_{\| r} \int \mathrm{D} \hat{r} \exp \left(-\sum_{\gamma=1}^{r} \frac{C_{\gamma}}{2}\left(\hat{v}\left(\mathbf{x}_{\| \gamma}\right)-\hat{r}\right)^{2}\right)
\end{array}
$$

Now we try to perform the integral over $\hat{r}$ and remember that $\mathrm{D} \hat{r}=\mathrm{d} \hat{r} / V^{n+1}$ :

$$
\begin{aligned}
& \int \mathrm{D} \hat{r} \exp \left(-\sum_{\gamma=1}^{r} \frac{C_{\gamma}}{2}\left(\hat{v}\left(\mathbf{x}_{\| \gamma}\right)-\hat{r}\right)^{2}\right) \\
& =\int \mathrm{D} \hat{r} \exp \left(-\sum_{\gamma=1}^{r} \frac{C_{\gamma}}{2}\left(\hat{v}^{2}\left(\mathbf{x}_{\| \gamma}\right)-2 \hat{r} \hat{v}\left(\mathbf{x}_{\| \gamma}\right)+\hat{r}^{2}\right)\right) \\
& =\exp \left(-\sum_{\gamma=1}^{r} \frac{C_{\gamma}}{2} \hat{v}^{2}\left(\mathbf{x}_{\| \gamma}\right)\right) \int \mathrm{D} \hat{r} \exp \left(-\frac{C}{2} \hat{r}^{2}+\hat{r} \sum_{\gamma=1}^{r} C_{\gamma} \hat{v}\left(\mathbf{x}_{\| \gamma}\right)\right) \\
& =\exp \left(-\sum_{\gamma=1}^{r} \frac{C_{\gamma}}{2} \hat{v}^{2}\left(\mathbf{x}_{\| \gamma}\right)\right) \frac{1}{V^{n+1}}\left(\frac{2 \pi}{C}\right)^{D(n+1) / 2} \exp \left(\frac{1}{2 C}\left(\sum_{\gamma=1}^{r} C_{\gamma} \hat{v}\left(\mathbf{x}_{\| \gamma}\right)\right)^{2}\right) \\
& =\frac{1}{V^{n+1}}\left(\frac{2 \pi}{C}\right)^{D(n+1) / 2} \exp \left(-\frac{1}{2 C}\left\{C \sum_{\gamma=1}^{r} C_{\gamma} \hat{v}^{2}\left(\mathbf{x}_{\| \gamma}\right)-\left(\sum_{\gamma=1}^{r} C_{\gamma} \hat{v}\left(\mathbf{x}_{\| \gamma}\right)\right)^{2}\right\}\right)
\end{aligned}
$$

Have a look at the term in the curly brackets \{\} :

$$
\begin{aligned}
C & \sum_{\gamma=1}^{r} C_{\gamma} \hat{v}^{2}\left(\mathbf{x}_{\| \gamma}\right)-\left(\sum_{\gamma=1}^{r} C_{\gamma} \hat{v}\left(\mathbf{x}_{\| \gamma}\right)\right)^{2} \\
= & \sum_{\gamma, \delta=1}^{r} C_{\gamma} C_{\delta} \hat{v}^{2}\left(\mathbf{x}_{\| \gamma}\right)-\sum_{\gamma, \delta=1}^{r} C_{\gamma} C_{\delta} \hat{v}\left(\mathbf{x}_{\| \gamma}\right) \hat{v}\left(\mathbf{x}_{\| \delta}\right) \\
= & \sum_{\gamma, \delta=1}^{r} C_{\gamma} C_{\delta} \hat{v}\left(\mathbf{x}_{\| \gamma}\right)\left(\hat{v}\left(\mathbf{x}_{\| \gamma}\right)-\hat{v}\left(\mathbf{x}_{\| \delta}\right)\right) \\
= & \sum_{\gamma<\delta} C_{\gamma} C_{\delta}\left(\hat{v}\left(\mathbf{x}_{\| \gamma}\right)-\hat{v}\left(\mathbf{x}_{\| \delta}\right)\right)\left(\hat{v}\left(\mathbf{x}_{\| \gamma}\right)-\hat{v}\left(\mathbf{x}_{\| \delta}\right)\right) \\
= & \sum_{\gamma<\delta} C_{\gamma} C_{\delta}\left(\hat{v}\left(\mathbf{x}_{\| \gamma}\right)-\hat{v}\left(\mathbf{x}_{\| \delta}\right)\right)^{2} \\
= & \sum_{\gamma<\delta} C_{\gamma} C_{\delta}\left(\left(u_{\perp}\left(\mathbf{x}_{\| \gamma}\right)-u_{\perp}\left(\mathbf{x}_{\| \delta}\right)\right)^{2}+\left(\mathbf{x}_{\| \gamma}-\mathbf{x}_{\| \delta}\right)^{2}\right)
\end{aligned}
$$


Hence Eq. (B.56) becomes:

$$
\begin{aligned}
& \int \mathrm{D} \hat{r} \exp \left(-\sum_{\gamma=1}^{r} \frac{C_{\gamma}}{2}\left(\hat{v}\left(\mathbf{x}_{\| \gamma}\right)+\hat{r}\right)^{2}\right)=\frac{1}{V^{n+1}}\left(\frac{2 \pi}{C}\right)^{D(n+1) / 2} \\
& \quad \times \exp \left(-\frac{1}{2 C}\left\{\sum_{\gamma<\delta} C_{\gamma} C_{\delta}\left(\left(u_{\perp}\left(\mathbf{x}_{\| \gamma}\right)-u_{\perp}\left(\mathbf{x}_{\| \delta}\right)\right)^{2}+\left(\mathbf{x}_{\| \gamma}-\mathbf{x}_{\| \delta}\right)^{2}\right)\right\}\right)
\end{aligned}
$$

and we can plug that in Eq. (B.55) to get:

$$
\begin{aligned}
& A_{r} /\left(\frac{Q^{r} V^{r n}}{(2 \pi)^{D r(n+1)}(n+1)^{D r / 2}} \frac{1}{V^{n+1}}\right) \\
& =\int_{\xi_{1}^{2}, \ldots, \xi_{r}^{2}}\left(\prod_{\gamma=1}^{r}\left(2 \pi C_{\gamma}\right)^{D(n+1) / 2}\right)\left(\frac{2 \pi}{C}\right)^{D(n+1) / 2} \\
& \quad \times \int \mathrm{d} \mathbf{x}_{\| 1} \cdots \mathrm{d}_{\| r} \exp \left(-\frac{1}{2 C} \sum_{\gamma<\delta} C_{\gamma} C_{\delta}\left(\mathbf{x}_{\| \gamma}-\mathbf{x}_{\| \delta}\right)^{2}\right) \\
& \quad \times \exp \left(-\frac{1}{2 C} \sum_{\gamma<\delta} C_{\gamma} C_{\delta}\left(u_{\perp}\left(\mathbf{x}_{\| \gamma}\right)-u_{\perp}\left(\mathbf{x}_{\| \delta}\right)\right)^{2}\right)
\end{aligned}
$$

In the last line one can see, as one would expect, that strong deformations, meaning a high $\left(u_{\perp}\left(\mathbf{x}_{\| \gamma}\right)-u_{\perp}\left(\mathbf{x}_{\| \delta}\right)\right)^{2}$, will have a high energy cost; unless $\mathbf{x}_{\| \gamma}$ and $\mathbf{x}_{\| \delta}$ are far away from each other, then the contribution will be suppressed by the exponential in the second last line and the magnitude of $\left(u_{\perp}\left(\mathbf{x}_{\| \gamma}\right)-u_{\perp}\left(\mathbf{x}_{\| \delta}\right)\right)^{2}$ does not matter.

As already mentioned, we are only interested in the lowest order of the deformation, $\mathcal{O}\left(\partial^{2} u_{\perp}^{2}\right)$, and in this spirit we make an expansion in $u_{\perp}$ :

$$
\begin{aligned}
u_{\perp}\left(\mathbf{x}_{\| \gamma}\right)-u_{\perp}\left(\mathbf{x}_{\| \delta}\right) & \\
= & u_{\perp}\left(\mathbf{x}_{\| \gamma}\right)-\left(u_{\perp}\left(\mathbf{x}_{\| \gamma}\right)+\sum_{\tau=1}^{D} \frac{\partial u_{\perp}\left(\mathbf{x}_{\| \gamma}\right)}{\partial x_{\| \gamma}^{(\tau)}}\left(x_{\| \delta}^{(\tau)}-x_{\| \gamma}^{(\tau)}\right)\right)+\mathcal{O}\left(\partial^{2} u_{\perp}\right) \\
& =\sum_{\tau=1}^{D} \frac{\partial u_{\perp}\left(\mathbf{x}_{\| \gamma}\right)}{\partial x_{\| \gamma}^{(\tau)}}\left(x_{\| \gamma}^{(\tau)}-x_{\| \delta}^{(\tau)}\right)+\mathcal{O}\left(\partial^{2} u_{\perp}\right)
\end{aligned}
$$

where $x_{\| \gamma}^{(\tau)}$ is the $\tau$-th spatial component of $\mathbf{x}_{\| \gamma}$. We furthermore define $u_{\perp}^{(\alpha, \nu)}\left(\mathbf{x}_{\| \gamma}\right)$ as the $\nu$-th spatial component in replica $\alpha$ of $u_{\perp}\left(\mathbf{x}_{\| \gamma}\right)$ and obtain:

$$
\begin{aligned}
& \left(u_{\perp}\left(\mathbf{x}_{\| \gamma}\right)-u_{\perp}\left(\mathbf{x}_{\| \delta}\right)\right)^{2} \\
& \quad=\sum_{\alpha, \nu} \sum_{\tau, \sigma} \frac{\partial u_{\perp}^{(\alpha, \nu)}\left(\mathbf{x}_{\| \gamma}\right)}{\partial x_{\| \gamma}^{(\tau)}} \frac{\partial u_{\perp}^{(\alpha, \nu)}\left(\mathbf{x}_{\| \gamma}\right)}{\partial x_{\| \gamma}^{(\sigma)}}\left(x_{\| \gamma}^{(\tau)}-x_{\| \delta}^{(\tau)}\right)\left(x_{\| \gamma}^{(\sigma)}-x_{\| \delta}^{(\sigma)}\right)+\mathcal{O}\left(\partial^{4} u_{\perp}^{2}\right)
\end{aligned}
$$


and therefore:

$$
\begin{aligned}
& \exp \left(-\frac{1}{2 C} \sum_{\gamma<\delta} C_{\gamma} C_{\delta}\left(u_{\perp}\left(\mathbf{x}_{\| \gamma}\right)-u_{\perp}\left(\mathbf{x}_{\| \delta}\right)\right)^{2}\right) \\
& =1-\frac{1}{2 C} \sum_{\gamma<\delta} C_{\gamma} C_{\delta} \sum_{\alpha, \nu} \sum_{\tau, \sigma} \frac{\partial u_{\perp}^{(\alpha, \nu)}\left(\mathbf{x}_{\| \gamma}\right)}{\partial x_{\| \gamma}^{(\tau)}} \frac{\partial u_{\perp}^{(\alpha, \nu)}\left(\mathbf{x}_{\| \gamma}\right)}{\partial x_{\| \gamma}^{(\sigma)}}\left(x_{\| \gamma}^{(\tau)}-x_{\| \delta}^{(\tau)}\right)\left(x_{\| \gamma}^{(\sigma)}-x_{\| \delta}^{(\sigma)}\right) \\
& \quad+\mathcal{O}\left(\partial^{4} u_{\perp}^{2}\right) .
\end{aligned}
$$

With the expanded $u_{\perp}$ and omitting the $\mathcal{O}\left(\partial^{4} u_{\perp}^{2}\right)$, Eq. (B.59) becomes:

$$
\begin{aligned}
& A_{r} /\left(\frac{Q^{r} V^{(r-1) n-1}}{(2 \pi)^{D r(n+1)}(n+1)^{D r / 2}}(2 \pi)^{D r(n+1) / 2+D(n+1) / 2}\right) \\
& =\int_{\xi_{1}^{2}, \ldots, \xi_{r}^{2}}\left(\prod_{\gamma=1}^{r} C_{\gamma}\right)^{D(n+1) / 2} \frac{1}{C^{D(n+1) / 2}} \\
& \times \int \mathrm{d} \mathbf{x}_{\| 1} \cdots \mathrm{d} \mathbf{x}_{\| r} \exp \left(-\frac{1}{2 C} \sum_{\gamma<\delta} C_{\gamma} C_{\delta}\left(\mathbf{x}_{\| \gamma}-\mathbf{x}_{\| \delta}\right)^{2}\right) \\
& \quad \times\left(1-\frac{1}{2 C} \sum_{\gamma<\delta} C_{\gamma} C_{\delta} \sum_{\alpha, \nu} \sum_{\tau, \sigma} \frac{\partial u_{\perp}^{(\alpha, \nu)}\left(\mathbf{x}_{\| \gamma}\right)}{\partial x_{\| \gamma}^{(\tau)}} \frac{\partial u_{\perp}^{(\alpha, \nu)}\left(\mathbf{x}_{\| \gamma}\right)}{\partial x_{\| \gamma}^{(\sigma)}}\left(x_{\| \gamma}^{(\tau)}-x_{\| \delta}^{(\tau)}\right)\left(x_{\| \gamma}^{(\sigma)}-x_{\| \delta}^{(\sigma)}\right)\right)
\end{aligned}
$$

When we look at the last two lines, we realize that the expression is symmetric with respect to interchanging any two $\gamma$ and $\delta$. Therefore, after performing the integral $\int \mathrm{d} \mathbf{x}_{\| 1} \cdots \mathrm{d} \mathbf{x}_{\| r}$, each summand of the sum $\sum_{\gamma<\delta}$ in the last line will have the same result. Since there are $r(r-1) / 2$ ways to choose $1 \leq \gamma<\delta \leq r$, we can replace the sum by $r(r-1) / 2$ and take $\gamma=r, \delta=1$ :

$$
\begin{aligned}
& A_{r} /\left(\frac{Q^{r} V^{(r-1) n-1}}{(2 \pi)^{D(r-1)(n+1) / 2}(n+1)^{D r / 2}}\right)=\int_{\xi_{1}^{2}, \ldots, \xi_{r}^{2}}\left(\frac{\prod_{\gamma=1}^{r} C_{\gamma}}{C}\right)^{D(n+1) / 2} \\
& \times \int \mathrm{d} \mathbf{x}_{\| 1} \cdots \mathrm{d} \mathbf{x}_{\| r} \exp \left(-\frac{1}{2 C} \sum_{\gamma<\delta} C_{\gamma} C_{\delta}\left(\mathbf{x}_{\| \gamma}-\mathbf{x}_{\| \delta}\right)^{2}\right) \\
& \times\left(1-\frac{r(r-1)}{2} \frac{C_{r} C_{1}}{2 C} \sum_{\alpha, \nu} \sum_{\tau, \sigma} \frac{\partial u_{\perp}^{(\alpha, \nu)}\left(\mathbf{x}_{\| r}\right)}{\partial x_{\| r}^{(\tau)}} \frac{\partial u_{\perp}^{(\alpha, \nu)}\left(\mathbf{x}_{\| r}\right)}{\partial x_{\| r}^{(\sigma)}}\left(x_{\| r}^{(\tau)}-x_{\| 1}^{(\tau)}\right)\left(x_{\| r}^{(\sigma)}-x_{\| 1}^{(\sigma)}\right)\right)
\end{aligned}
$$

It is clear that we will not be able to perform the integral over $\mathbf{x}_{\| r}$, because we do not know the actual form of $u_{\perp}^{(\alpha, \nu)}\left(\mathbf{x}_{\| r}\right)$ at this point. However, for $\mathbf{x}_{\| 1}, \ldots, \mathbf{x}_{\|(r-1)}$ we can try to perform the integral. Let us first make the substitution $\mathbf{y}_{\| \gamma}=\mathbf{x}_{\| \gamma}-\mathbf{x}_{\| r}$ for $\gamma<r$ and make the simplifying definition

$$
U_{\tau \sigma}\left(\mathbf{x}_{\| r}\right):=\frac{r(r-1)}{2} \frac{C_{r} C_{1}}{2 C} \sum_{\alpha, \nu} \frac{\partial u_{\perp}^{(\alpha, \nu)}\left(\mathbf{x}_{\| r}\right)}{\partial x_{\| r}^{(\tau)}} \frac{\partial u_{\perp}^{(\alpha, \nu)}\left(\mathbf{x}_{\| r}\right)}{\partial x_{\| r}^{(\sigma)}}
$$


Hence we get:

$$
\begin{aligned}
A_{r} /\left(\frac{Q^{r} V^{(r-1) n-1}}{(2 \pi)^{D(r-1)(n+1) / 2}(n+1)^{D r / 2}}\right) \\
\quad=\int_{\xi_{1}^{2}, \ldots, \xi_{r}^{2}}\left(\frac{\prod_{\gamma=1}^{r} C_{\gamma}}{C}\right)^{D(n+1) / 2} \int \mathrm{d} \mathbf{x}_{\| r} B_{r}\left(\mathbf{x}_{\| r}\right)
\end{aligned}
$$

with

$$
\begin{aligned}
B_{r}\left(\mathbf{x}_{\| r}\right)=\int \mathrm{d} \mathbf{y}_{\| 1} & \cdots \mathbf{y}_{\|(r-1)} \exp \left(-\frac{1}{2 C}\left(\sum_{\gamma<\delta<r} C_{\gamma} C_{\delta}\left(\mathbf{y}_{\| \gamma}-\mathbf{y}_{\| \delta}\right)^{2}+\sum_{\gamma<r} C_{\gamma} C_{r} \mathbf{y}_{\| \gamma}^{2}\right)\right) \\
& \times\left(1-\sum_{\tau, \sigma} U_{\tau \sigma}\left(\mathbf{x}_{\| r}\right) y_{\| 1}^{(\tau)} y_{\| 1}^{(\sigma)}\right)
\end{aligned}
$$

which we try to calculate now. Let us have a look at the sum $\sum_{\tau, \sigma}$ in the last line. For $\tau \neq \sigma$, integration over $\mathbf{y}_{\| 1}$ yields zero due to point symmetry of the integrand.

To perform the integral over $\mathbf{y}_{\| 1}, \ldots, \mathbf{y}_{\|(r-1)}$, we can write the sums in the exponential such that it takes the form of a well known Gaussian integral:

$$
\begin{aligned}
T & =\sum_{\gamma<\delta<r} C_{\gamma} C_{\delta}\left(\mathbf{y}_{\| \gamma}-\mathbf{y}_{\| \delta}\right)^{2}+\sum_{\gamma<r} C_{\gamma} C_{r} \mathbf{y}_{\| \gamma}^{2} \\
& =\sum_{\gamma<\delta<r} C_{\gamma} C_{\delta} \mathbf{y}_{\| \gamma}^{2}-2 \sum_{\gamma<\delta<r} C_{\gamma} C_{\delta} \mathbf{y}_{\| \gamma} \mathbf{y}_{\| \delta}+\sum_{\gamma<\delta<r} C_{\gamma} C_{\delta} \mathbf{y}_{\| \delta}^{2}+\sum_{\gamma<r} C_{\gamma} C_{r} \mathbf{y}_{\| \gamma}^{2}
\end{aligned}
$$

The first and third term can be combined to one sum over $\gamma \neq \delta$. The second sum can also be changed to a sum over $\gamma \neq \delta$, eliminating the factor of 2 :

$$
T=\sum_{\substack{\gamma \neq \delta \\ \gamma, \delta<r}} C_{\gamma} C_{\delta} \mathbf{y}_{\| \gamma}^{2}-\sum_{\substack{\gamma \neq \delta \\ \gamma, \delta<r}} C_{\gamma} C_{\delta} \mathbf{y}_{\| \gamma} \mathbf{y}_{\| \delta}+C_{r} \sum_{\gamma<r} C_{\gamma} \mathbf{y}_{\| \gamma}^{2}
$$

We can also include the term $\gamma=\delta$ in the first and second sum. Since they make the same contribution in both sums, with opposite sign, they cancel out exactly.

$$
\begin{aligned}
T & =\sum_{\gamma, \delta<r} C_{\gamma} C_{\delta} \mathbf{y}_{\| \gamma}^{2}-\sum_{\gamma, \delta<r} C_{\gamma} C_{\delta} \mathbf{y}_{\| \gamma} \mathbf{y}_{\| \delta}+C_{r} \sum_{\gamma<r} C_{\gamma} \mathbf{y}_{\| \gamma}^{2} \\
& =\left(C-C_{r}\right) \sum_{\gamma<r} C_{\gamma} \mathbf{y}_{\| \gamma}^{2}-\sum_{\gamma, \delta<r} C_{\gamma} C_{\delta} \mathbf{y}_{\| \gamma} \mathbf{y}_{\| \delta}+C_{r} \sum_{\gamma<r} C_{\gamma} \mathbf{y}_{\| \gamma}^{2} \\
& =C \sum_{\gamma<r} C_{\gamma} \mathbf{y}_{\| \gamma}^{2}-\sum_{\gamma, \delta<r} C_{\gamma} C_{\delta} \mathbf{y}_{\| \gamma} \mathbf{y}_{\| \delta}
\end{aligned}
$$


Hence we can write Eq. (B.66b) as:

$B_{r}\left(\mathbf{x}_{\| r}\right)=\int \mathrm{d} \mathbf{y}_{\| 1} \cdots \mathbf{y}_{\|(r-1)} \exp \left(-\sum_{\gamma, \delta<r} M_{\gamma \delta} \mathbf{y}_{\| \gamma} \mathbf{y}_{\| \delta}\right)\left(1-\sum_{\tau=1}^{D} U_{\tau \tau}\left(\mathbf{x}_{\| r}\right)\left(y_{\| 1}^{(\tau)}\right)^{2}\right)$

with a Matrix $\quad M_{\gamma \delta}=\frac{\delta_{\gamma \delta} C C_{\gamma}-C_{\gamma} C_{\delta}}{2 C}=\delta_{\gamma \delta} \frac{C_{\gamma}}{2}-\frac{C_{\gamma} C_{\delta}}{2 C}$

To perform the integral, we introduce a $D$-dimensional vector $\mathbf{b}_{\|}=\left(b_{\|}^{(1)}, \ldots, b_{\|}^{(D)}\right)^{T}$ coupling to $\mathbf{y}_{\| 1}$ and rewrite that expression as:

$$
\begin{aligned}
B_{r}\left(\mathbf{x}_{\| r}\right)=\lim _{\mathbf{b}_{\|} \rightarrow \mathbf{0}}( & \left.1-\sum_{\tau=1}^{D} U_{\tau \tau}\left(\mathbf{x}_{\| r}\right)\left(\frac{\partial}{\partial b_{\|}^{(\tau)}}\right)^{2}\right) \\
& \int \mathrm{d} \mathbf{y}_{\| 1} \cdots \mathbf{y}_{\|(r-1)} \exp \left(-\sum_{\gamma, \delta<r} M_{\gamma \delta} \mathbf{y}_{\| \gamma} \mathbf{y}_{\| \delta}+\mathbf{b}_{\| \mathbf{y}_{\| 1}}\right)
\end{aligned}
$$

Now, the Gaussian in the second line can easily be calculated:

$$
\begin{gathered}
\int \mathrm{d}_{\| 1} \cdots \mathrm{d}_{\|(r-1)} \exp \left(-\sum_{\gamma, \delta<r} M_{\gamma \delta} \mathbf{y}_{\| \gamma} \mathbf{y}_{\| \delta}+\mathbf{b}_{\|} \mathbf{y}_{\| 1}\right) \\
=\frac{\pi^{D(r-1) / 2}}{(\operatorname{det} M)^{D / 2}} \exp \left(\frac{1}{4} \mathbf{b}_{\|}^{2} M_{11}^{-1}\right),
\end{gathered}
$$

with the determinant $\operatorname{det} M$ and inverse $M^{-1}$ of the matrix $M$ (and $M_{11}^{-1}$ being the entry at position $(1,1)$ of $\left.M^{-1}\right)$. With that, Eq. (B.71) becomes:

$$
\begin{aligned}
B_{r}\left(\mathbf{x}_{\| r}\right) & =\frac{\pi^{D(r-1) / 2}}{(\operatorname{det} M)^{D / 2}} \lim _{\mathbf{b}_{\|} \rightarrow \mathbf{0}}\left(1-\sum_{\tau=1}^{D} U_{\tau \tau}\left(\mathbf{x}_{\| r}\right)\left(\frac{\partial}{\partial b_{\|}^{(\tau)}}\right)^{2}\right) \exp \left(\frac{1}{4} \mathbf{b}_{\|}^{2} M_{11}^{-1}\right) \\
& =\frac{\pi^{D(r-1) / 2}}{(\operatorname{det} M)^{D / 2}} \lim _{\mathbf{b}_{\|} \rightarrow \mathbf{0}}\left(1-\sum_{\tau=1}^{D} U_{\tau \tau}\left(\mathbf{x}_{\| r}\right) \frac{\partial}{\partial b_{\|}^{(\tau)}} \frac{2}{4} b_{\|}^{(\tau)} M_{11}^{-1} \exp \left(\frac{1}{4} \mathbf{b}_{\|}^{2} M_{11}^{-1}\right)\right) \\
& =\frac{\pi^{D(r-1) / 2}}{(\operatorname{det} M)^{D / 2}}\left(1-\frac{M_{11}^{-1}}{2} \sum_{\tau=1}^{D} U_{\tau \tau}\left(\mathbf{x}_{\| r}\right)\right)
\end{aligned}
$$

To calculate the determinant and inverse of $M$, we take advantage of its form $M=A+\mathbf{x y}^{T}$ (i.e. $\left.M_{\gamma \delta}=A_{\gamma \delta}+x_{\gamma} y_{\delta}\right)$ with a "simple" matrix $A$, and use the relations [Brookes, 2009]:

$$
\begin{aligned}
\operatorname{det}\left(A+\mathbf{x y}^{T}\right) & =\operatorname{det}(A)\left(1+\mathbf{y}^{T} A^{-1} \mathbf{x}\right), \\
\left(A+\mathbf{x y}^{T}\right)^{-1} & =A^{-1}-\frac{A^{-1} \mathbf{x y}^{T} A^{-1}}{1+\mathbf{y}^{T} A^{-1} \mathbf{x}} .
\end{aligned}
$$


In our case, we choose:

$$
\begin{aligned}
& A_{\gamma \delta}=\delta_{\gamma \delta} \frac{C_{\gamma}}{2}, \\
& x_{\gamma}=-\frac{C_{\gamma}}{2 C} \text { and } \quad y_{\delta}=C_{\delta},
\end{aligned}
$$

with $1 \leq \gamma, \delta \leq r-1$. Applying relation (B.74a), we easily find:

$$
\begin{aligned}
\operatorname{det} M & =\frac{C_{1} \cdots C_{r-1}}{2^{r-1}}\left(1+\sum_{\gamma=1}^{r-1} \frac{-C_{\gamma}}{2 C} \frac{2}{C_{\gamma}} C_{\gamma}\right) \\
& =\frac{C_{1} \cdots C_{r-1}}{2^{r-1}}\left(1-\frac{1}{C}\left(C-C_{r}\right)\right)=\frac{1}{2^{r-1} C} \prod_{\gamma=1}^{r} C_{\gamma}
\end{aligned}
$$

And by applying (B.74b) one gets (after a similar calculation, which is left to the eager reader(s)):

$$
M_{\gamma \delta}^{-1}=\frac{2 \delta_{\gamma \delta}}{C_{\gamma}}-\frac{2}{C_{r}} \quad \text { and hence } \quad M_{11}^{-1}=\frac{2\left(C_{1}+C_{r}\right)}{C_{1} C_{r}}
$$

Now we can plug these two results (B.76) into Eq. (B.73) and get the result for $B_{r}\left(\mathbf{x}_{\| r}\right):$

$$
\begin{aligned}
B_{r}\left(\mathbf{x}_{\| r}\right) & =\frac{\pi^{D(r-1) / 2}}{(\operatorname{det} M)^{D / 2}}\left(1-\frac{M_{11}^{-1}}{2} \sum_{\tau=1}^{D} U_{\tau \tau}\left(\mathbf{x}_{\| r}\right)\right) \\
& =\pi^{D(r-1) / 2}\left(\frac{\prod_{\gamma=1}^{r} C_{\gamma}}{2^{r-1} C}\right)^{-D / 2}\left(1-\frac{C_{1}+C_{r}}{C_{1} C_{r}} \sum_{\tau=1}^{D} U_{\tau \tau}\left(\mathbf{x}_{\| r}\right)\right)
\end{aligned}
$$

To get $A_{r}$, we can now plug this result for $B_{r}\left(\mathbf{x}_{\| r}\right)$ back into Eq. (B.66a):

$$
\begin{aligned}
& A_{r} /\left(\frac{Q^{r} V^{(r-1) n-1}}{(2 \pi)^{D(r-1)(n+1) / 2}(n+1)^{D r / 2}}(2 \pi)^{D(r-1) / 2}\right) \\
& =\int_{\xi_{1}^{2}, \ldots, \xi_{r}^{2}}\left(\frac{\prod_{\gamma=1}^{r} C_{\gamma}}{C}\right)^{D(n+1) / 2-D / 2} \int \mathrm{d} \mathbf{x}_{\| r}\left(1-\frac{C_{1}+C_{r}}{C_{1} C_{r}} \sum_{\tau=1}^{D} U_{\tau \tau}\left(\mathbf{x}_{\| r}\right)\right)
\end{aligned}
$$

To simplify the notation, we can change the integration variable $\mathbf{x}_{\| r}$ to just $\mathbf{x}_{\|}$and insert the definition of $U_{\tau \tau}\left(\mathbf{x}_{\|}\right)$, see Eq. (B.65):

$$
\begin{aligned}
& A_{r} /\left(\frac{Q^{r} V^{(r-1) n-1}}{(2 \pi)^{D(r-1) n / 2}(n+1)^{D r / 2}}\right)=\int_{\xi_{1}^{2}, \ldots, \xi_{r}^{2}}\left(\frac{\prod_{\gamma=1}^{r} C_{\gamma}}{C}\right)^{D n / 2} \\
& \quad \times \int \mathrm{d} \mathbf{x}_{\|}\left(1-\frac{C_{1}+C_{r}}{C_{1} C_{r}} \cdot r(r-1) \frac{C_{r} C_{1}}{4 C} \sum_{\tau} \sum_{\alpha, \nu} \frac{\partial u_{\perp}^{(\alpha, \nu)}\left(\mathbf{x}_{\|}\right)}{\partial x_{\|}^{(\tau)}} \frac{\partial u_{\perp}^{(\alpha, \nu)}\left(\mathbf{x}_{\|}\right)}{\partial x_{\|}^{(\tau)}}\right)
\end{aligned}
$$


After canceling $C_{1} C_{r}$, we realize that we can arbitrarily swap $C_{1}$ and $C_{r}$ in the last line with any other $C_{\gamma}$, because the rest of the expression is symmetric with respect to $C_{\gamma}$. So we can even change $C_{1} \rightarrow\left(C_{1}+\ldots+C_{r}\right) / r=C / r$ and also $C_{r} \rightarrow C / r$. Doing that, we get:

$$
\begin{aligned}
& A_{r} /\left(\frac{Q^{r} V^{(r-1) n-1}}{(2 \pi)^{D(r-1) n / 2}(n+1)^{D r / 2}}\right)=\int_{\xi_{1}^{2}, \ldots, \xi_{r}^{2}}\left(\frac{\prod_{\gamma=1}^{r} C_{\gamma}}{C}\right)^{D n / 2} \\
& \quad \times \int \mathrm{d} \mathbf{x}_{\|}\left(1-\frac{2 C}{r} \cdot r(r-1) \frac{1}{4 C} \sum_{\tau} \sum_{\alpha, \nu} \frac{\partial u_{\perp}^{(\alpha, \nu)}\left(\mathbf{x}_{\|}\right)}{\partial x_{\|}^{(\tau)}} \frac{\partial u_{\perp}^{(\alpha, \nu)}\left(\mathbf{x}_{\|}\right)}{\partial x_{\|}^{(\tau)}}\right)
\end{aligned}
$$

Finally we plug in definitions (B.53) and (B.54) and end up with the result for $A_{r}$ (but keep in mind that it is exact only to lowest order in the displacement field $u_{\perp}$ and its derivative):

$$
\begin{aligned}
A_{r}=Q^{r} \frac{1}{(n+1)^{D(r-1) / 2}}\left(\frac{V}{(2 \pi)^{D / 2}}\right)^{(r-1) n} \\
\quad \times \int_{0}^{\infty} \mathrm{d} \xi_{1}^{2} \mathcal{P}\left(\xi_{1}^{2}\right) \cdots \mathrm{d} \xi_{r}^{2} \mathcal{P}\left(\xi_{r}^{2}\right)\left(\frac{\prod_{\gamma=1}^{r} \frac{1}{a^{2}+\xi_{\gamma}^{2}}}{\sum_{\gamma=1}^{r} \frac{1}{a^{2}+\xi_{\gamma}^{2}}}\right)^{D n / 2} \\
\quad \times\left(1-\frac{r-1}{2} \int \mathrm{Dx}_{\|} \sum_{\alpha} \sum_{\tau, \nu}\left(\frac{\partial u_{\perp}^{(\alpha, \nu)}\left(\mathbf{x}_{\|}\right)}{\partial x_{\|}^{(\tau)}}\right)^{2}\right)
\end{aligned}
$$

with

$$
\mathrm{D} \mathbf{x}_{\|}=\frac{\mathrm{d} \mathbf{x}_{\|}}{V(n+1)^{D / 2}} .
$$

\section{B.5.4 Insertion of the Order Parameter in $f_{n+1}\{\Omega\}$}

In the previous section we calculated the one-particle partition function $\mathfrak{z}$, using Ansatz (B.39) for the order parameter. To get the full replica free energy, we still have to insert the Ansatz into the first part of Eq. (B.41a):

$$
f_{n+1}\left\{\bar{\Omega}_{u}^{\prime}\right\}=-\frac{\phi^{n} \mu^{2} Q^{2}}{2}+\frac{\phi^{n} \mu^{2}}{2} \underbrace{\sum_{\hat{q} \in \operatorname{HRS} \vee\{\hat{0}\}} \Delta(\hat{q})\left|\bar{\Omega}_{u}^{\prime}(\hat{q})\right|^{2}}_{=: S_{2}}-\ln \mathfrak{z} .
$$

For the cross-link term $\Delta(\hat{q})$ we note that

$$
\Delta(\hat{q})=\exp \left(-\frac{\hat{q}^{2} a^{2}}{2}\right)=\exp \left(-\frac{\hat{q}^{2} a^{2} / 2+(-\hat{q})^{2} a^{2} / 2}{2}\right)=\Delta_{a^{2} / 2}(\hat{q}) \Delta_{a^{2} / 2}(-\hat{q}) .
$$


where the symbol $\Delta_{a^{2} / 2}(\hat{q})$ is the same as the original $\Delta(\hat{q})$, only with cross-link strength $a^{2} \rightarrow a^{2} / 2$. Thus we can rewrite the sum

$$
\begin{aligned}
S_{2} & =\sum_{\hat{q} \in \operatorname{HRS} \vee\{\hat{0}\}} \Delta(\hat{q})\left|\bar{\Omega}_{u}^{\prime}(\hat{q})\right|^{2}=\sum_{\hat{q} \in \operatorname{HRS} \vee\{\hat{0}\}} \Delta_{a^{2} / 2}(\hat{q}) \Delta_{a^{2} / 2}(-\hat{q}) \bar{\Omega}_{u}^{\prime}(\hat{q}) \bar{\Omega}_{u}^{\prime}(-\hat{q}) \\
& =\sum_{\substack{\hat{q}_{1}, \hat{q}_{2} \in \\
\operatorname{HRS} \vee\{\hat{0}\}}} \delta_{\hat{q}_{1}+\hat{q}_{2}, \hat{0}} \Delta_{a^{2} / 2}\left(\hat{q}_{1}\right) \Delta_{a^{2} / 2}\left(\hat{q}_{2}\right) \bar{\Omega}_{u}^{\prime}\left(\hat{q}_{1}\right) \bar{\Omega}_{u}^{\prime}\left(\hat{q}_{2}\right) \\
& =\int \operatorname{D} \hat{r} \sum_{\substack{\left.\hat{q}_{1}, \hat{q}_{2} \in \\
\operatorname{HRS} \vee \hat{0}\right\}}} \mathrm{e}^{-\mathrm{i}\left(\hat{q}_{1}+\hat{q}_{2}\right) \hat{r}} \Delta_{a^{2} / 2}\left(\hat{q}_{1}\right) \Delta_{a^{2} / 2}\left(\hat{q}_{2}\right) \bar{\Omega}_{u}^{\prime}\left(\hat{q}_{1}\right) \bar{\Omega}_{u}^{\prime}\left(\hat{q}_{2}\right) \\
& =\int \operatorname{D} \hat{r}\left\{\sum_{\hat{q} \in \operatorname{HRS} \vee\{\hat{0}\}} \Delta_{a^{2} / 2}(\hat{q}) \bar{\Omega}_{u}^{\prime}(\hat{q}) \mathrm{e}^{-\mathrm{i} \hat{q} \hat{r}}\right\}^{2} .
\end{aligned}
$$

Comparing that expression to Eq. (B.43), we note that $S_{2}$ has the same form as $A_{2}$ with $a^{2} \rightarrow a^{2} / 2$. Therefore, using the result for $A_{2}$, Eq. (B.81), we can immediately write down the solution:

$$
\begin{aligned}
S_{2}=\frac{Q^{2}}{(n+1)^{D / 2}}\left(\frac{V}{(2 \pi)^{D / 2}}\right)^{n} \int_{0}^{\infty} & \mathrm{d} \xi_{1}^{2} \mathcal{P}\left(\xi_{1}^{2}\right) \mathrm{d} \xi_{2}^{2} \mathcal{P}\left(\xi_{2}^{2}\right)\left(\frac{\frac{1}{a^{2} / 2+\xi_{1}^{2}} \cdot \frac{1}{a^{2} / 2+\xi_{2}^{2}}}{\frac{1}{a^{2} / 2+\xi_{1}^{2}}+\frac{1}{a^{2} / 2+\xi_{2}^{2}}}\right)^{D n / 2} \\
& \times\left(1-\frac{1}{2} \int \mathrm{D} \mathbf{x}_{\|} \sum_{\alpha} \sum_{\tau, \nu}\left(\frac{\partial u_{\perp}^{(\alpha, \nu)}\left(\mathbf{x}_{\|}\right)}{\partial x_{\|}^{(\tau)}}\right)^{2}\right)
\end{aligned}
$$

\section{B.5.5 Recomposing the Replica Free Energy}

We now recompose the expanded replica free energy $f_{n+1}\left\{\bar{\Omega}_{u}\right\}$ from the different components we calculated throughout the section B.5: In terms of $S_{2}$ and $A_{r}$, it is given by Eqs. (B.83) and (B.42):

$$
\begin{aligned}
f_{n+1}\left\{\bar{\Omega}_{u}\right\} & =-\frac{\phi^{n} \mu^{2} Q^{2}}{2}+\frac{\phi^{n} \mu^{2}}{2} S_{2}-\ln \mathfrak{z} . \\
\mathfrak{z} & =\exp \left(-\phi^{n} \mu^{2} Q\right) \sum_{r=0}^{\infty} \frac{\left(\phi^{n} \mu^{2}\right)^{r}}{r !} A_{r}
\end{aligned}
$$

Since we want to take the limit $n \rightarrow 0$, as seen in Eq. (2.10), we only keep the various parts up to linear order in $n$ and omit higher orders. Therefore it is necessary to keep in mind that the sum $\sum_{\alpha=0}^{n}\left(\partial u_{\perp}^{(\alpha, \nu)}\left(\mathbf{x}_{\|}\right) / \partial x_{\|}^{(\tau)}\right)^{2}$ is already of the order $\mathcal{O}(n)$, see Eq. (2.51). We furthermore need:

$$
\begin{aligned}
(n+1)^{-D(r-1) / 2} & =1-n \frac{D(r-1)}{2}+\mathcal{O}\left(n^{2}\right) \\
\mathcal{A}^{n} & =1+n \ln \mathcal{A}+\mathcal{O}\left(n^{2}\right)
\end{aligned}
$$


With that, we get for $r \geq 2$ :

$$
\begin{aligned}
\frac{A_{r}}{Q^{r}}= & \left\{1-n \frac{D(r-1)}{2}\right\} \\
& \times \int_{\xi_{1}^{2}, \ldots, \xi_{r}^{2}}\left\{1+n \ln \left(\left(\frac{V}{(2 \pi)^{D / 2}}\right)^{r-1}\left(\frac{\prod_{\gamma=1}^{r} C_{\gamma}}{C}\right)^{D / 2}\right)\right\} \\
& \times\left\{1-\frac{r-1}{2} \int \mathrm{D} \mathbf{x}_{\|} \sum_{\alpha=0}^{n} \sum_{\tau, \nu}\left(\frac{\partial u_{\perp}^{(\alpha, \nu)}\left(\mathbf{x}_{\|}\right)}{\partial x_{\|}^{(\tau)}}\right)^{2}\right\}+\mathcal{O}\left(n^{2}\right) \\
= & -n \frac{D(r-1)}{2}+n \underbrace{}_{\xi_{1}^{2}, \ldots, \xi_{r}^{2}} \ln \left(\left(\frac{V}{(2 \pi)^{D / 2}}\right)^{r-1}\left(\frac{\prod_{\gamma=1}^{r} C_{\gamma}}{C}\right)^{D / 2}\right) \\
& -\frac{r-1}{2} \int \mathrm{Dx}_{\|} \sum_{\alpha=0}^{n} \sum_{\tau, \nu}\left(\frac{\partial u_{\perp}^{(\alpha, \nu)}\left(\mathbf{x}_{\|}\right)}{\partial x_{\|}^{(\tau)}}\right)^{2}+\mathcal{O}\left(n^{2}\right)
\end{aligned}
$$

With that expression (for $r \geq 2$ ) and $A_{0}=1, A_{1}=Q$, we can calculate $\mathfrak{z}$ :

$$
\begin{aligned}
& \frac{\mathfrak{z}}{\exp \left(-\phi^{n} \mu^{2} Q\right)}= \\
& =1+\phi^{n} \mu^{2} Q+\sum_{r=2}^{\infty} \frac{\left(\phi^{n} \mu^{2} Q\right)^{r}}{r !} \frac{A_{r}}{Q^{r}} \\
& =\sum_{r=0}^{\infty} \frac{\left(\phi^{n} \mu^{2} Q\right)^{r}}{r !}+n \cdot \sum_{r=2}^{\infty} \frac{\left(\phi^{n} \mu^{2} Q\right)^{r}}{r !}\left(-\frac{D(r-1)}{2}+\Xi_{r, a^{2}}\right) \\
& -\sum_{r=2}^{\infty} \frac{\left(\phi^{n} \mu^{2} Q\right)^{r}}{r !} \cdot \frac{r-1}{2} \int \mathrm{D} \mathbf{x}_{\|} \sum_{\alpha=0}^{n} \sum_{\tau, \nu}\left(\frac{\partial u_{\perp}^{(\alpha, \nu)}\left(\mathbf{x}_{\|}\right)}{\partial x_{\|}^{(\tau)}}\right)^{2}+\mathcal{O}\left(n^{2}\right) .
\end{aligned}
$$

To perform the sum over $r$, we need the relations:

$$
\begin{aligned}
\sum_{r=0}^{\infty} \frac{\left(\phi^{n} \mu^{2} Q\right)^{r}}{r !} & =\exp \left(\phi^{n} \mu^{2} Q\right) \\
\sum_{r=2}^{\infty} \frac{\left(\phi^{n} \mu^{2} Q\right)^{r}}{r !}(r-1) & =1+\sum_{r=0}^{\infty} \frac{\left(\phi^{n} \mu^{2}\right)^{r}}{r !}(r-1) \\
\text { Eq. (D.2) } & \stackrel{=}{=} 1+\left(\phi^{n} \mu^{2} Q-1\right) \exp \left(\phi^{n} \mu^{2} Q\right)
\end{aligned}
$$

and get:

$$
\begin{aligned}
\mathfrak{z}=1 & +n\left(-\frac{D}{2}\left(\exp \left(-\phi^{\not \alpha} \mu^{2} Q\right)+\phi^{\not x} \mu^{2} Q-1\right)+\exp \left(-\phi^{\not x} \mu^{2} Q\right) \sum_{r=2}^{\infty} \frac{\left(\phi^{\not x} \mu^{2} Q\right)^{r}}{r !} \Xi_{r, a^{2}}\right) \\
& -\frac{\exp \left(-\phi^{\not x} \mu^{2} Q\right)+\phi^{\not x} \mu^{2} Q-1}{2} \int \mathrm{D} \mathbf{x}_{\|} \sum_{\alpha=0}^{n} \sum_{\tau, \nu}\left(\frac{\partial u_{\perp}^{(\alpha, \nu)}\left(\mathbf{x}_{\|}\right)}{\partial x_{\|}^{(\tau)}}\right)^{2}+\mathcal{O}\left(n^{2}\right),
\end{aligned}
$$


whereby the crossed-out terms are omitted, since they make contributions of at least quadratic order in $n$. In terms of expansion in $n, \mathfrak{z}$ has the simple form $\mathfrak{z}=1+n \cdot$ const. $+\mathcal{O}\left(n^{2}\right)$. Hence the logarithm can easily be taken and is $\ln \mathfrak{z}=$ $n \cdot$ const. $+\mathcal{O}\left(n^{2}\right)=\mathfrak{z}-1+\mathcal{O}\left(n^{2}\right)$

$$
\begin{aligned}
\ln \mathfrak{z}=n & \left(-\frac{D}{2}\left(\exp \left(-\mu^{2} Q\right)+\mu^{2} Q-1\right)+\exp \left(-\mu^{2} Q\right) \sum_{r=2}^{\infty} \frac{\left(\mu^{2} Q\right)^{r}}{r !} \Xi_{r, a^{2}}\right) \\
& -\frac{\exp \left(-\mu^{2} Q\right)+\mu^{2} Q-1}{2} \int \mathrm{D} \mathbf{x}_{\|} \sum_{\alpha=0}^{n} \sum_{\tau, \nu}\left(\frac{\partial u_{\perp}^{(\alpha, \nu)}\left(\mathbf{x}_{\|}\right)}{\partial x_{\|}^{(\tau)}}\right)^{2}+\mathcal{O}\left(n^{2}\right),
\end{aligned}
$$

Analogously, we perform the expansion of $S_{2}$ up to linear order in $n$. Remembering that $S_{2}$ equals $A_{2}$ with $a^{2} \rightarrow a^{2} / 2$, we can use Eq. (B.90) to write down the result immediately:

$$
\begin{aligned}
\frac{S_{2}}{Q^{2}}=1 & +n\left(-\frac{D}{2}+\Xi_{2, a^{2} / 2}\right) \\
& -\frac{1}{2} \int \mathrm{D} \mathbf{x}_{\|} \sum_{\alpha=0}^{n} \sum_{\tau, \nu}\left(\frac{\partial u_{\perp}^{(\alpha, \nu)}\left(\mathbf{x}_{\|}\right)}{\partial x_{\|}^{(\tau)}}\right)^{2}+\mathcal{O}\left(n^{2}\right)
\end{aligned}
$$

Now, we are able to write down the result for the replica free energy, according to Eq. (B.87a):

$$
\begin{aligned}
f_{n+1}\left\{\bar{\Omega}_{u}\right\} & =-\frac{\phi^{n} \mu^{2} Q^{2}}{2}+\frac{\phi^{n} \mu^{2} Q^{2}}{2} \\
& +n \frac{\phi^{x} \mu^{2} Q^{2}}{2}\left(-\frac{D}{2}+\Xi_{2, a^{2} / 2}\right)-\frac{\phi^{\not x} \mu^{2} Q^{2}}{2} \cdot \frac{1}{2} \int \mathrm{D} \mathbf{x}_{\|} \sum_{\alpha=0}^{n} \sum_{\tau, \nu}\left(\frac{\partial u_{\perp}^{(\alpha, \nu)}\left(\mathbf{x}_{\|}\right)}{\partial x_{\|}^{(\tau)}}\right)^{2} \\
& -n\left(-\frac{D}{2}\left(\exp \left(-\mu^{2} Q\right)+\mu^{2} Q-1\right)+\exp \left(-\mu^{2} Q\right) \sum_{r=2}^{\infty} \frac{\left(\mu^{2} Q\right)^{r}}{r !} \Xi_{r, a^{2}}\right) \\
& +\frac{\exp \left(-\mu^{2} Q\right)+\mu^{2} Q-1}{2} \int \operatorname{Dx}_{\|} \sum_{\alpha=0}^{n} \sum_{\tau, \nu}\left(\frac{\partial u_{\perp}^{(\alpha, \nu)}\left(\mathbf{x}_{\|}\right)}{\partial x_{\|}^{(\tau)}}\right)^{2}+\mathcal{O}\left(n^{2}\right)
\end{aligned}
$$

The fact the the two constant terms in the first line cancel out is no coincidence: As already seen in Eq. (2.28), $\lim _{n \rightarrow 0} f_{n+1}\{\Omega\}$ must be zero, because the sum over the higher replica sector does not contain any elements, since the constraint that at least two components are non-zero cannot be fulfilled, if there is only one component. Thus, there cannot be a constant term and the first terms must be at least linear in $n$.

The above result can be written in a very concise way as:

$$
f_{n+1}\left\{\bar{\Omega}_{u}\right\}=f_{n+1}^{\mathrm{sp}}+\frac{1}{N} \cdot \frac{G}{2} \int \mathrm{d} \mathbf{x}_{\|} \sum_{\alpha=0}^{n} \sum_{\tau, \nu}\left(\frac{\partial u_{\perp}^{(\alpha, \nu)}\left(\mathbf{x}_{\|}\right)}{\partial x_{\|}^{(\tau)}}\right)^{2}+\mathcal{O}\left(n^{2}\right)
$$


whereby the first term, $f_{n+1}^{\mathrm{sp}}$, is the saddle-point value of $f_{n+1}\{\Omega\}$ :

$$
\begin{aligned}
f_{n+1}^{\mathrm{sp}}=n\left\{\frac{D}{2}\left(\exp \left(-\mu^{2} Q\right)+\mu^{2} Q-1-\frac{\mu^{2} Q^{2}}{2}\right)\right. & \\
+ & \left.\frac{\mu^{2} Q^{2}}{2} \Xi_{2, a^{2} / 2}-\exp \left(-\mu^{2} Q\right) \sum_{r=2}^{\infty} \frac{\left(\mu^{2} Q\right)^{r}}{r !} \Xi_{r, a^{2}}\right\}
\end{aligned}
$$

and the second term describes the cost of fluctuations (i.e. shear deformations) around the saddle point, with the shear modulus

$$
G=\left(\exp \left(-\mu^{2} Q\right)+\mu^{2} Q-1-\frac{\mu^{2} Q^{2}}{2}\right) n_{0}
$$

\section{B.5.6 Analysis of the quantity $\Xi_{r, a^{2}}$}

In this section we have to look at the term $\Xi_{r, a^{2}}$ that was defined in Eq. (B.90) and still appears in the saddle point value of the replica free energy Eq. (B.98b).

$$
\begin{aligned}
\Xi_{r, a^{2}}:= & \int_{\xi_{1}^{2}, \ldots, \xi_{r}^{2}} \ln \left(\left(\frac{V}{(2 \pi)^{D / 2}}\right)^{r-1}\left(\frac{\prod_{\gamma=1}^{r}\left(a^{2}+\xi_{\gamma}^{2}\right)^{-1}}{\sum_{\gamma=1}^{r}\left(a^{2}+\xi_{\gamma}^{2}\right)^{-1}}\right)^{D / 2}\right) \\
= & \int_{\xi_{1}^{2}, \ldots, \xi_{r}^{2}} \ln \left(\left(\frac{N}{(2 \pi)^{D / 2}}\right)^{r-1}\left(\frac{\prod_{\gamma=1}^{r} \frac{n_{0}^{2 / D}}{a^{2}+\xi_{\gamma}^{2}}}{\sum_{\gamma=1}^{r} \frac{n_{0}^{2 / D}}{a^{2}+\xi_{\gamma}^{2}}}\right)^{D / 2}\right) \\
= & (r-1) \ln N-\frac{D(r-1)}{2} \ln (2 \pi) \\
& +\frac{D}{2} \sum_{\gamma=1}^{r} \int_{\xi_{1}^{2}, \ldots, \xi_{r}^{2}} \ln \left(\frac{n_{0}^{2 / D}}{a^{2}+\xi_{\gamma}^{2}}\right)-\frac{D}{2} \int_{\xi_{1}^{2}, \ldots, \xi_{r}^{2}} \ln \left(\sum_{\gamma=1}^{r} \frac{n_{0}^{2 / D}}{a^{2}+\xi_{\gamma}^{2}}\right) \\
= & (r-1) \ln N-\frac{D(r-1)}{2} \ln (2 \pi) \\
& +\frac{D r}{2} \int_{\xi^{2}} \ln \left(\frac{n_{0}^{2 / D}}{a^{2}+\xi^{2}}\right)-\frac{D}{2} \int_{\xi_{1}^{2}, \ldots, \xi_{r}^{2}} \ln \left(\sum_{\gamma=1}^{r} \frac{n_{0}^{2 / D}}{a^{2}+\xi_{\gamma}^{2}}\right)
\end{aligned}
$$

Having the thermodynamic limit in mind, we assume that all internal length scales are small compared to the system size:

$$
V^{1 / D} \gg \xi, a \Longleftrightarrow N \gg n_{0} \xi^{D}, n_{0} a^{D}
$$

In this limit, only the first term of $\Xi_{r, a^{2}}$ in Eq. (B.99) remains:

$$
\Xi_{r, a^{2}}=(r-1) \ln N+\mathcal{O}\left(N^{0}\right) .
$$




\section{B.6 Auxiliary Calculations for the Results Section}

\section{B.6.1 Distribution of the Number of Cross-Links}

Here we are interested in the distribution of the number $M$ of cross-links in the system, and in particular its mean. It is possible to calculate expectation values of the form $[M(M-1) \cdots(M-J+1)]=: \mathcal{M}_{J}$ using the Deam-Edwards distribution Eq. (2.8) for the disorder average $[\cdot]$. In doing so, we recall the simplifying definition $\tilde{\mu}^{2}=\mu^{2} /\left(2 n_{0} a^{D}(2 \pi)^{D / 2}\right)$ :

$$
\begin{aligned}
& \mathcal{M}_{J}=[M(M-1) \cdots(M-J+1)]= \\
& =\frac{1}{\mathcal{Z}_{1}} \sum_{M=0}^{\infty} \sum_{\substack{i_{1}, \ldots, i_{M}, j_{1}, \ldots, j_{M}=1}}^{N} \frac{\left(\tilde{\mu}^{2}\right)^{M}}{M !} Z\left(\left\{i_{e}, j_{e}\right\}_{e=1}^{M}\right) \cdot M(M-1) \cdots(M-J+1)
\end{aligned}
$$

The summands for $M=0,1, \ldots, J-1$ obviously yield zero and can be excluded from the first sum. For $M \geq J$, we can write:

$$
M(M-1) \cdots(M-J+1)=\frac{M !}{(M-J) !},
$$

and therefore:

$$
\mathcal{M}_{J}=\frac{1}{\mathcal{Z}_{1}} \sum_{M=J}^{\infty} \sum_{\substack{i_{1}, \ldots, i_{M}, j_{1}, \ldots, j_{M}=1}}^{N} \frac{\left(\tilde{\mu}^{2}\right)^{M}}{M !} \frac{M T !}{(M-J) !} Z\left(\left\{i_{e}, j_{e}\right\}_{e=1}^{M}\right)
$$

Now we start the sum at $M=0$ again, whereby all occurrences of $M$ shift to $M+J$ :

$$
\mathcal{M}_{J}=\frac{1}{\mathcal{Z}_{1}} \sum_{M=0}^{\infty} \sum_{\substack{i_{1}, \ldots, i_{M+J}, j_{1}, \ldots, j_{M+J}=1}}^{N} \frac{\left(\tilde{\mu}^{2}\right)^{M+J}}{(M+J-J) !} Z\left(\left\{i_{e}, j_{e}\right\}_{e=1}^{M+J}\right)
$$

Now we write out the partition function explicitly:

$$
\mathcal{M}_{J}=\frac{1}{\mathcal{Z}_{1}} \int \mathrm{D} \Gamma \mathrm{e}^{-H_{\mathrm{ev}}\left(\left\{\mathbf{r}_{j}\right\}\right)} \sum_{M=0}^{\infty} \sum_{\substack{i_{1}, \ldots, i_{M+J}, j_{1}, \ldots, j_{M+J}=1}}^{N} \frac{\left(\tilde{\mu}^{2}\right)^{M+J}}{M !} \exp \left(-\frac{1}{2 a^{2}} \sum_{e=1}^{M+J}\left(\mathbf{r}_{i_{e}}-\mathbf{r}_{j_{e}}\right)^{2}\right)
$$


where $\int \mathrm{D} \Gamma$ denotes integration over all particle positions $\int \prod_{j=1}^{N} \mathrm{D} \mathbf{r}_{j}$. This form can be written in a much more convenient way:

$$
\begin{aligned}
\mathcal{M}_{J}= & \frac{1}{\mathcal{Z}_{1}} \int \mathrm{D} \Gamma \mathrm{e}^{-H_{\mathrm{ev}}\left(\left\{\mathbf{r}_{j}\right\}\right)} \sum_{M=0}^{\infty} \frac{1}{M !}\left(\tilde{\mu}^{2} \sum_{i, j=1}^{N} \exp \left(-\frac{1}{2 a^{2}}\left(\mathbf{r}_{i}-\mathbf{r}_{j}\right)^{2}\right)\right)^{M+J} \\
= & \frac{1}{\mathcal{Z}_{1}} \int \mathrm{D} \Gamma \mathrm{e}^{-H_{\mathrm{ev}}\left(\left\{\mathbf{r}_{j}\right\}\right)}\left(\tilde{\mu}^{2} \sum_{i, j=1}^{N} \exp \left(-\frac{\left(\mathbf{r}_{i}-\mathbf{r}_{j}\right)^{2}}{2 a^{2}}\right)\right)^{J} \\
& \times \exp \left(\tilde{\mu}^{2} \sum_{i, j=1}^{N} \exp \left(-\frac{\left(\mathbf{r}_{i}-\mathbf{r}_{j}\right)^{2}}{2 a^{2}}\right)\right) \\
= & \frac{\left(\tilde{\mu}^{2}\right)^{J}}{\mathcal{Z}_{1}}\left(\frac{\partial}{\partial \tilde{\mu}^{2}}\right)^{J} \int \mathrm{D} \Gamma \mathrm{e}^{-H_{\mathrm{ev}}\left(\left\{\mathbf{r}_{j}\right\}\right)} \exp \left(\tilde{\mu}^{2} \sum_{i, j=1}^{N} \exp \left(-\frac{\left(\mathbf{r}_{i}-\mathbf{r}_{j}\right)^{2}}{2 a^{2}}\right)\right)
\end{aligned}
$$

The integral in the last line is exactly $\lim _{n \rightarrow 0} \mathcal{Z}_{n+1}=\mathcal{Z}_{1}$, as we can confirm with Eq. (2.14):

$$
\mathcal{M}_{J}=\frac{\left(\tilde{\mu}^{2}\right)^{J}}{\mathcal{Z}_{1}}\left(\frac{\partial}{\partial \tilde{\mu}^{2}}\right)^{J} \mathcal{Z}_{1}
$$

and since $\tilde{\mu}^{2}$ is proportional to $\mu^{2}$ :

$$
\mathcal{M}_{J}=\frac{\left(\mu^{2}\right)^{J}}{\mathcal{Z}_{1}}\left(\frac{\partial}{\partial \mu^{2}}\right)^{J} \mathcal{Z}_{1}
$$

Calculating $\mathcal{Z}_{1}$ is very easy: We know that $\lim _{n \rightarrow 0} f_{n+1}\{\Omega\}=0$, because it involves only sums over the higher replica sector (see Eq. (2.28)), confirmed by the result (2.53) in Sec. 2.7. Therefore we just need to look at Eq. (2.27a), in the limit $n \rightarrow 0$ :

$$
\mathcal{Z}_{1}=\exp \left(-N f_{0}\right) \times 1
$$

with $f_{0}$ given in Eq. (2.19):

$$
\mathcal{Z}_{1}=\exp \left(-N\left(-\frac{\mu^{2}}{2}+\frac{\lambda n_{0}}{2}\right)\right)=\exp \left(\frac{N \mu^{2}}{2}-\frac{N \lambda n_{0}}{2}\right)
$$

It is noteworthy that the limit of very large $\lambda$ seems to be an issue here. However, since $\mathcal{Z}_{1}$ is both in the numerator and the denominator in Eq. (B.109), the dependence on $\lambda$ will cancel out and the limit $\lambda \rightarrow \infty$ is possible. Now we can take the derivative:

$$
\left(\frac{\partial}{\partial \mu^{2}}\right)^{J} \mathcal{Z}_{1}=\left(\frac{N}{2}\right)^{J} \mathcal{Z}_{1}
$$

and with Eq. (B.109) we get:

$$
\mathcal{M}_{J}=[M(M-1) \cdots(M-J+1)]=\left(\frac{\mu^{2} N}{2}\right)^{J}
$$

This result finds further use in Sec. 2.9.1. 


\section{B.6.2 Shear modulus}

In Sec. 2.9.3 we wrote the shear modulus as:

$$
\frac{G}{n_{0} k_{\mathrm{B}} T}=\frac{\mu^{2}}{2}-1+(1-Q)-\frac{\mu^{2}}{2}(1-Q)^{2} .
$$

We want to find a power series for that expression, for large cross-link densities $\mu^{2}$. Therefore we need the expansion of $(1-Q)$ and $(1-Q)^{2}$. An expression for $(1-Q)$ is given in Eq. (2.73):

$$
(1-Q)=\mathrm{e}^{-\mu^{2}} \sum_{k=0}^{\infty} \frac{(k+1)^{k-1}}{k !}\left(\mu^{2} \mathrm{e}^{-\mu^{2}}\right)^{k}
$$

For $(1-Q)^{2}$, we use Eq. (B.128) derived in Appendix B.6.3 for the square of the sum:

$$
\begin{aligned}
(1-Q)^{2} & =\mathrm{e}^{-2 \mu^{2}}\left(\sum_{k=0}^{\infty} \frac{(k+1)^{k-1}}{k !}\left(\mu^{2} \mathrm{e}^{-\mu^{2}}\right)^{k}\right)^{2} \\
& =\mathrm{e}^{-2 \mu^{2}} \sum_{k=0}^{\infty} \frac{2(k+2)^{k-1}}{k !}\left(\mu^{2} \mathrm{e}^{-\mu^{2}}\right)^{k}
\end{aligned}
$$

With that we can calculate

$$
\begin{aligned}
(1- & Q)-\frac{\mu^{2}}{2}(1-Q)^{2}= \\
& =\mathrm{e}^{-\mu^{2}} \sum_{k=0}^{\infty} \frac{(k+1)^{k-1}}{k !}\left(\mu^{2} \mathrm{e}^{-\mu^{2}}\right)^{k}-\frac{\mu^{2} \mathrm{e}^{-2 \mu^{2}}}{\not 2} \sum_{k=0}^{\infty} \frac{\mathfrak{2}(k+2)^{k-1}}{k !}\left(\mu^{2} \mathrm{e}^{-\mu^{2}}\right)^{k} \\
& =\mathrm{e}^{-\mu^{2}}\left(\sum_{k=0}^{\infty} \frac{(k+1)^{k-1}}{k !}\left(\mu^{2} \mathrm{e}^{-\mu^{2}}\right)^{k}-\sum_{k=0}^{\infty} \frac{(k+2)^{k-1}}{k !}\left(\mu^{2} \mathrm{e}^{-\mu^{2}}\right)^{k+1}\right) \\
& =\mathrm{e}^{-\mu^{2}}\left(\sum_{k=0}^{\infty} \frac{(k+1)^{k-1}}{k !}\left(\mu^{2} \mathrm{e}^{-\mu^{2}}\right)^{k}-\sum_{k=1}^{\infty} \frac{k(k+1)^{k-2}}{k !}\left(\mu^{2} \mathrm{e}^{-\mu^{2}}\right)^{k}\right) \\
& =\mathrm{e}^{-\mu^{2}} \sum_{k=0}^{\infty} \frac{(k+1)^{k-1}-k(k+1)^{k-2}}{k !}\left(\mu^{2} \mathrm{e}^{-\mu^{2}}\right)^{k} \\
& =\mathrm{e}^{-\mu^{2}} \sum_{k=0}^{\infty} \frac{(k+1)^{k-2}(k+1-\not k)}{k !}\left(\mu^{2} \mathrm{e}^{-\mu^{2}}\right)^{k}
\end{aligned}
$$

And hence:

$$
(1-Q)-\frac{\mu^{2}}{2}(1-Q)^{2}=\mathrm{e}^{-\mu^{2}} \sum_{k=0}^{\infty} \frac{(k+1)^{k-2}}{k !}\left(\mu^{2} \mathrm{e}^{-\mu^{2}}\right)^{k}
$$

In Sec. 2.9.3, we plug this expression into Eq. (2.77) to obtain the expansion of the shear modulus $G$ for $\mu^{2} \gg 1$, far from the sol-gel transition. 


\section{B.6.3 Square of the expansion for $(1-Q)$}

The goal is to calculate the square of a series of the form:

$$
\tilde{W}(x):=\sum_{k=0}^{\infty} \frac{(k+1)^{k-1}}{k !} x^{k} .
$$

This function $\tilde{W}(x)$ is related to the Lambert $W$ function by $\tilde{W}(x)=-W(-x) / x$. To find the square of $\tilde{W}(x)$, we use the relation

$$
\begin{aligned}
\left(\sum_{k=0}^{\infty} a_{k} x^{k}\right)^{2} & =\sum_{k=0}^{\infty} \sum_{k^{\prime}=0}^{\infty} a_{k} x^{k} a_{k^{\prime}} x^{k^{\prime}}=\sum_{k=0}^{\infty} b_{k} x^{k} \\
\text { with } \quad b_{k} & =\sum_{l=0}^{k} a_{l} a_{k-l} .
\end{aligned}
$$

In our case:

$$
a_{k}=\frac{(k+1)^{k-1}}{k !}
$$

and therefore

$$
\begin{aligned}
b_{k} & =\sum_{l=0}^{k} \frac{(l+1)^{l-1}}{l !} \frac{(k-l+1)^{k-l-1}}{(k-l) !} \\
& =\frac{1}{k !} \sum_{l=0}^{k}\left(\begin{array}{l}
k \\
l
\end{array}\right)(l+1)^{l-1}(k-l+1)^{k-l-1}
\end{aligned}
$$

The calculation of $b_{k}$ is a little bit tedious, but not difficult. Let us first consider the following relation [Knuth, 1973, p. 56]:

$$
(x+y)^{r}=x \sum_{l=0}^{r}\left(\begin{array}{l}
r \\
l
\end{array}\right)(x-l z)^{l-1}(y+l z)^{r-l} \quad \text { for } \quad r \geq 0, x \neq 0
$$

We choose $z=-1, x=1, y=k+1$ and $r=k, k-1$ to get:

$$
\begin{aligned}
(k+2)^{k} & =\sum_{l=0}^{k}\left(\begin{array}{c}
k \\
l
\end{array}\right)(1+l)^{l-1}(k-l+1)^{k-l} \\
(k+2)^{k-1} & =\sum_{l=0}^{k-1}\left(\begin{array}{c}
k-1 \\
l
\end{array}\right)(1+l)^{l-1}(k-l+1)^{k-l-1} .
\end{aligned}
$$

With the help of the two above equations, we can calculate:

$$
\begin{aligned}
& 2(k+2)^{k-1} \\
= & (k+2)^{k-1}(k+2-k) \\
= & (k+2)^{k}-k(k+2)^{k-1}
\end{aligned}
$$




$$
\begin{aligned}
= & \sum_{l=0}^{k}\left(\frac{k !}{l !(k-l) !}(1+l)^{l-1}(k-l+1)^{k-l}\right) \\
& \quad-k \sum_{l=0}^{k-1}\left(\frac{(k-1) !}{l !(k-l-1) !}(1+l)^{l-1}(k-l+1)^{k-l-1}\right) \\
= & \sum_{l=0}^{k-1}\left(\frac{k !}{l !(k-l) !}(1+l)^{l-1}(k-l+1)^{k-l}-\frac{k !}{l !(k-l-1) !}(1+l)^{l-1}(k-l+1)^{k-l-1}\right) \\
& \quad+(1+k)^{k-1} \\
= & \sum_{l=0}^{k-1}\left(\left\{\frac{k !}{l !(k-l) !}(1+l)^{l-1}(k-l+1)^{k-l-1}\right\}\{k-l+1-(k-l)\}\right)+(1+k)^{k-1} \\
= & \sum_{l=0}^{k-1}\left(\frac{k !}{l !(k-l) !}(1+l)^{l-1}(k-l+1)^{k-l-1}\right)+(1+k)^{k-1} \\
= & \sum_{l=0}^{k}\left(\begin{array}{l}
k \\
l
\end{array}\right)(1+l)^{l-1}(k-l+1)^{k-l-1}
\end{aligned}
$$

Comparing that relation with $b_{k}$ in Eq. (B.122), we can write down

$$
b_{k}=\frac{2(k+2)^{k-1}}{k !} \text {. }
$$

Putting $b_{k}$ into Eq. (B.120b), we get the desired result:

$$
\tilde{W}^{2}(x)=\left(\sum_{k=0}^{\infty} \frac{(k+1)^{k-1}}{k !} x^{k}\right)^{2}=\sum_{k=0}^{\infty} \frac{2(k+2)^{k-1}}{k !} x^{k}
$$

\section{B.7 The Order Parameter in the One Replica Sector}

In Sec. 2.6.2 we derived an Ansatz for the order parameter $\Omega(\hat{q})$ that allows shear fluctuations around the saddle point, see Eq. (2.45):

$$
\begin{gathered}
\bar{\Omega}_{u}(\hat{q})=(1-Q) \delta_{\hat{q}, \hat{0}}+Q \omega(\hat{q}) \int \mathrm{D} \mathbf{x}_{\|} \exp \left(\mathbf{i} \mathbf{q}_{\|} \mathbf{x}_{\|}+\mathrm{i} q_{\perp} u_{\perp}\left(\mathbf{x}_{\|}\right)\right) \\
\text {with } \quad \omega(\hat{q})=\int_{0}^{\infty} \mathrm{d} \xi^{2} \mathcal{P}\left(\xi^{2}\right) \exp \left(-\frac{\hat{q}^{2} \xi^{2}}{2}\right) .
\end{gathered}
$$

For $\hat{q} \in 1 \mathrm{RS}$, this order parameter describes simple density fluctuations in the respective replica. Hence, if $u_{\perp}\left(\mathbf{x}_{\|}\right)$describes (volume conserving) shear deformations, one would expect $\Omega(\hat{q})$ to vanish. Here, we show that this is indeed the case. For $\hat{q}=(\mathbf{0}, \ldots, \mathbf{0}, \mathbf{q}, \mathbf{0}, \ldots, \mathbf{0})$, we obtain: 


$$
\begin{aligned}
& \mathbf{q}_{\|}=\frac{1}{\sqrt{n+1}} \mathbf{q} \\
& q_{\perp}=\hat{q}-\frac{1}{n+1}\left(\begin{array}{c}
\mathbf{q} \\
\vdots \\
\mathbf{q}
\end{array}\right)
\end{aligned}
$$

Hence, we can write Eq. (B.129b) in terms of q:

$$
\begin{aligned}
& \int \mathrm{d} \mathbf{x}_{\|} \exp \left(\mathbf{i} \mathbf{q}_{\|} \mathbf{x}_{\|}+\mathrm{i} q_{\perp} u_{\perp}\left(\mathbf{x}_{\|}\right)\right) \\
= & \int \mathrm{d} \mathbf{x}_{\|} \exp \left(\frac{\mathbf{i} \mathbf{q} \mathbf{x}_{\|}}{\sqrt{n+1}}+\mathrm{i} \mathbf{q u} \mathbf{u}_{\perp}^{(\alpha)}\left(\mathbf{x}_{\|}\right)-\frac{\mathrm{iq}}{n+1} \sum_{\beta=0}^{n} \mathbf{u}_{\perp}^{(\beta)}\left(\mathbf{x}_{\|}\right)\right)
\end{aligned}
$$

The last term vanishes, because by definition, the sum over the components of a $\perp$-vector is zero, $\sum_{\beta=0}^{n} \mathbf{u}_{\perp}^{(\beta)}\left(\mathbf{x}_{\|}\right)=\mathbf{0}$. Now we can introduce the new integration variable

$$
\begin{gathered}
\mathbf{x}_{\|}^{\prime}:=\mathbf{x}_{\|}+\sqrt{n+1} \mathbf{u}_{\perp}^{(\alpha)}\left(\mathbf{x}_{\|}\right) \\
\operatorname{det}\left(\frac{\partial \mathbf{x}_{\|}^{\prime}}{\partial \mathbf{x}_{\|}}\right)=\operatorname{det}\left(\mathbb{1}+\sqrt{n+1} \frac{\partial \mathbf{u}_{\perp}^{(\alpha)}\left(\mathbf{x}_{\|}\right)}{\partial \mathbf{x}_{\|}}\right),
\end{gathered}
$$

with which Eq. (B.131) becomes:

$$
\begin{aligned}
& \int \mathrm{d} \mathbf{x}_{\|} \exp \left(\mathbf{i} \mathbf{q}_{\|} \mathbf{x}_{\|}+\mathrm{i} q_{\perp} u_{\perp}\left(\mathbf{x}_{\|}\right)\right) \\
= & \int \mathrm{d} \mathbf{x}_{\|}^{\prime} \exp \left(\frac{\mathbf{i} \mathbf{q} \mathbf{x}_{\|}^{\prime}}{\sqrt{n+1}}\right)\left|\operatorname{det}\left(\mathbb{1}+\sqrt{n+1} \frac{\partial \mathbf{u}_{\perp}^{(\alpha)}\left(\mathbf{x}_{\|}\right)}{\partial \mathbf{x}_{\|}}\right)\right|
\end{aligned}
$$

The prefactor $\sqrt{n+1}$ in the determinant is just coming from the difference between the usual vector $\mathbf{x}$ and the parallel-vector $\mathbf{x}_{\|}=\sqrt{n+1} \mathbf{x}$. We only allow incompressible deformations (see Sec. 2.6.2), for which

$$
\operatorname{det}\left(\mathbb{1}+\sqrt{n+1} \frac{\partial \mathbf{u}_{\perp}^{(\alpha)}\left(\mathbf{x}_{\|}\right)}{\partial \mathbf{x}_{\|}}\right)=\operatorname{det}\left(\mathbb{1}+\frac{\partial \mathbf{u}_{\perp}^{(\alpha)}\left(\mathbf{x}_{\|}\right)}{\partial \mathbf{x}}\right) \equiv 1
$$

and hence:

$$
\begin{aligned}
& \int \mathrm{d} \mathbf{x}_{\|} \exp \left(\mathbf{i} \mathbf{q}_{\|} \mathbf{x}_{\|}+\mathrm{i} q_{\perp} u_{\perp}\left(\mathbf{x}_{\|}\right)\right) \\
= & \int \mathrm{d} \mathbf{x}_{\|}^{\prime} \exp \left(\mathbf{i} \mathbf{q}_{\|} \mathbf{x}_{\|}^{\prime}\right)=V_{\|} \delta_{\mathbf{q}_{\|}, \mathbf{0}}
\end{aligned}
$$

Therefore, for incompressible deformations and $\hat{q}$-vectors in the 1RS (which are non-zero), the order parameter becomes:

$$
\bar{\Omega}_{u}(\hat{q})=(1-Q) \delta_{\hat{q}, \hat{0}}+Q \delta_{\mathbf{q}_{\|}, \mathbf{0}}=0,
$$

as we already anticipated. 

ApPendix C

\section{Calculations for the Directed Polymer Model}

\section{Contents}

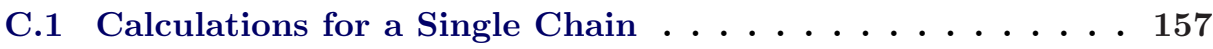

C.1.1 The Uncrosslinked Chain . . . . . . . . . . . . . . . 157

C.1.2 The Polymer Clamped in Space . . . . . . . . . . . . . . . 159

C.2 Examples of Single Chain Interactions . . . . . . . . . 160

C.3 Calculations for the Disorder-Averaged Free Energy . . . 162

C.3.1 Introduction of Replicas . . . . . . . . . . . . . . . . . 162

C.3.2 Introduction of the Replicated Density Field . . . . . . . . 163

C.3.3 The Hubbard-Stratonovich Transformation . . . . . . . . . . . 164

C.4 The Saddle-Point Equation with Ansatz . . . . . . . . 167

C.4.1 Expansion in $Q$ to Infinite Order . . . . . . . . . . . . 167

C.4.2 Expansion to Second Order in $Q \ldots \ldots$. . . . . . . . 169

C.5 Obtaining the Equation for the Localization Length $\ldots 173$

C.5.1 Normalization of Length Scales . . . . . . . . . . . . . 173

C.5.2 Laplace-Transformation of the Saddle Point Equation . . . . . 175

C.6 Calculation of a Correlator . . . . . . . . . . . 177

C.7 The Average Cross-Link Density . . . . . . . . . . . 180

\section{C.1 Calculations for a Single Chain}

\section{C.1.1 The Uncrosslinked Chain}

In Sec. 3.2.1 we defined the radius of gyration of an uncrosslinked polymer chain as

$$
\begin{aligned}
R_{\mathrm{g}}^{2} & =\left\langle\frac{1}{2 L^{2}} \int \mathrm{d} z_{1} \mathrm{~d} z_{2}\left(\mathbf{r}\left(z_{1}\right)-\mathbf{r}\left(z_{2}\right)\right)^{2}\right\rangle_{H_{\text {align }, 1}} \\
& =\frac{1}{2 L^{2}} \int \mathcal{D} \mathbf{r}(z) \exp \left(-\frac{\sigma}{2} \int \mathrm{d} z \dot{\mathbf{r}}^{2}(z)\right) \int \mathrm{d} z_{1} \mathrm{~d} z_{2}\left(\mathbf{r}\left(z_{1}\right)-\mathbf{r}\left(z_{2}\right)\right)^{2}
\end{aligned}
$$


We discretize the functional integral into $Z_{L}$ points, such that the function $\mathbf{r}(z)$ is represented by $Z_{L}$ discrete vectors $\mathbf{r}_{1}, \ldots, \mathbf{r}_{Z_{L}}$. With the normalization (3.4) of the functional integral we get:

$$
R_{\mathrm{g}}^{2}=\frac{\frac{1}{2 Z_{L}^{2}} \int \mathrm{d} \mathbf{r}_{1} \cdots \mathrm{d} \mathbf{r}_{Z_{L}} \exp \left(-\frac{\sigma \cdot \Delta z}{2} \sum_{Z=1}^{Z_{L}-1} \frac{\left(\mathbf{r}_{Z+1}-\mathbf{r}_{Z}\right)^{2}}{\Delta z^{2}}\right) \sum_{Z_{1}, Z_{2}=1}^{Z_{L}}\left(\mathbf{r}_{Z_{1}}-\mathbf{r}_{Z_{2}}\right)^{2}}{\int \mathrm{d} \mathbf{r}_{1} \cdots \mathrm{d} \mathbf{r}_{Z_{L}} \exp \left(-\frac{\sigma \cdot \Delta z}{2} \sum_{Z=1}^{Z_{L}-1} \frac{\left(\mathbf{r}_{Z+1}-\mathbf{r}_{Z}\right)^{2}}{\Delta z^{2}}\right)}
$$

Now, instead of integrating over all positions of the chain segments $\mathbf{r}_{Z}$, we can integrate over the position of the bottom end of the chain $\mathbf{r}_{1}$ and over the position differences $\mathbf{t}_{Z}:=\mathbf{r}_{Z}-\mathbf{r}_{Z-1}$ for $Z=2, \ldots, Z_{L}$ :

$$
\begin{aligned}
& R_{\mathrm{g}}^{2}=\frac{\frac{1}{Z_{L}^{2}} \int \mathrm{d} \mathbf{r}_{1} \mathrm{~d} \mathbf{t}_{2} \cdots \mathrm{d} \mathbf{t}_{Z_{L}} \exp \left(-\frac{\sigma}{2 \Delta z} \sum_{Z=1}^{Z_{L}-1} \mathbf{t}_{Z+1}^{2}\right) \sum_{Z_{1}<Z_{2}}\left(\sum_{Z=Z_{1}+1}^{Z_{2}} \mathbf{t}_{Z}\right)^{2}}{\int \mathrm{d} \mathbf{r}_{1} \mathrm{~d} \mathbf{t}_{2} \cdots \mathrm{d} \mathbf{t}_{Z_{L}} \exp \left(-\frac{\sigma}{2 \Delta z} \sum_{Z=1}^{Z_{L}-1} \mathbf{t}_{Z+1}^{2}\right)} \\
& \int \mathrm{d} \mathbf{t}_{Z_{1}+1} \cdots \mathrm{d} \mathbf{t}_{Z_{2}} \exp \left(-\frac{\sigma}{2 \Delta z} \sum_{Z=Z_{1}+1}^{Z_{2}} \mathbf{t}_{Z}^{2}\right)\left(\sum_{Z=Z_{1}+1}^{Z_{2}} \mathbf{t}_{Z}^{2}+\sum_{Z, Z^{\prime} \neq Z_{1}+1}^{Z_{2}} \mathbf{t}_{Z^{\prime}} \mathbf{t}_{Z^{\prime}}\right)
\end{aligned}
$$

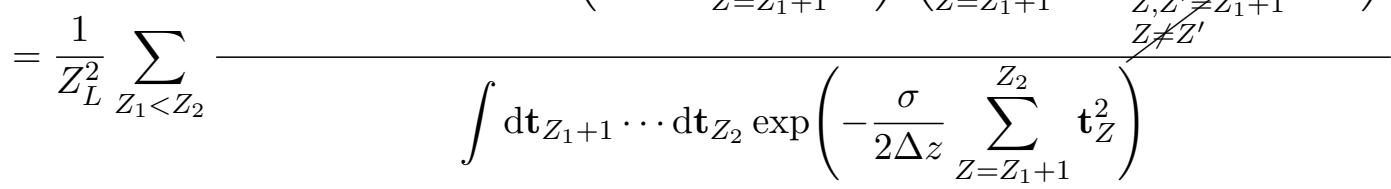

$$
\begin{aligned}
& =\frac{1}{Z_{L}^{2}} \sum_{Z_{1}<Z_{2}} \frac{\left(\frac{2 \pi \cdot \Delta z}{\sigma}\right)^{Z_{2}-Z_{1}} \frac{\Delta z \cdot D\left(Z_{2}-Z_{1}\right)}{2 \sigma}}{\left(\frac{2 \pi \cdot \Delta z}{\sigma}\right)^{Z_{2}-Z_{1}}} \\
& =\frac{D \Delta z}{2 Z_{L}^{2} \sigma} \sum_{Z_{1}<Z_{2}}\left(Z_{2}-Z_{1}\right) \\
& =\frac{D \Delta z}{2 Z_{L}^{2} \sigma}\left(\frac{1}{6}\left(Z_{L}^{2}-1\right) Z_{L}\right) \stackrel{\text { large }}{=} Z_{L} \frac{D \Delta z}{2 Z_{L}^{2} \sigma}\left(\frac{Z_{L}^{3}}{6}\right)=\frac{D}{2} \frac{Z_{L} \cdot \Delta z}{6 \sigma},
\end{aligned}
$$

and since $\Delta z=L / Z_{L}$ we get:

$$
R_{\mathrm{g}}^{2}=\frac{D}{6} \frac{L}{2 \sigma}
$$




\section{C.1.2 The Polymer Clamped in Space}

Let us have a closer look at the partition function. As in the previous section 3.2.1, we discretize the functional integral into integrals over the chain segments:

$$
\begin{aligned}
Z= & \int \mathcal{D} \mathbf{r}(z) \exp \left(-\frac{\sigma}{2} \int \mathrm{d} z \dot{\mathbf{r}}^{2}(z)-\frac{1}{2 a^{2}} \mathbf{r}^{2}\left(z_{1}\right)+\mathbf{b r}\left(z_{0}\right)\right) \\
= & \frac{\int \mathrm{d} \mathbf{r}_{1} \cdots \mathrm{d} \mathbf{r}_{Z_{L}} \exp \left(-\frac{\sigma \cdot \Delta z}{2} \sum_{Z=1}^{Z_{L}-1} \frac{\left(\mathbf{r}_{Z+1}-\mathbf{r}_{Z}\right)^{2}}{\Delta z^{2}}-\frac{\mathbf{r}_{Z_{1}}^{2}}{2 a^{2}}+\mathbf{b} \mathbf{r}_{Z_{0}}\right)}{\int \mathrm{d} \mathbf{r}_{1} \cdots \mathrm{d} \mathbf{r}_{Z_{L}} \exp \left(-\frac{\sigma \cdot \Delta z}{2} \sum_{Z=1}^{Z_{L}-1} \frac{\left(\mathbf{r}_{Z+1}-\mathbf{r}_{Z}\right)^{2}}{\Delta z^{2}}\right)}
\end{aligned}
$$

The denominator is easy to compute:

$$
\int \mathrm{d} \mathbf{r}_{1} \mathrm{~d} \mathbf{t}_{2} \cdots \mathrm{d} \mathbf{t}_{Z_{L}} \exp \left(-\frac{\sigma}{2 \Delta z} \sum_{Z=1}^{Z_{L}-1} \mathbf{t}_{Z+1}^{2}\right)=A\left(\frac{2 \pi \Delta z}{\sigma}\right)^{D\left(Z_{L}-1\right) / 2} .
$$

For the numerator we keep the integration variable $\mathbf{r}_{Z_{1}}$ and define $\mathbf{t}_{Z}=\mathbf{r}_{Z+1}-\mathbf{r}_{Z}$ for $1 \leq Z \leq Z_{L}-1$. This change of variables yields the following substitution scheme for $\mathbf{r}_{Z}$ :

$$
\begin{aligned}
\mathbf{r}_{Z_{1}-2} & =\mathbf{r}_{Z_{1}}-\mathbf{t}_{Z_{1}-1}-\mathbf{t}_{Z_{1}-2} \\
\mathbf{r}_{Z_{1}-1} & =\mathbf{r}_{Z_{1}}-\mathbf{t}_{Z_{1}-1} \\
\mathbf{r}_{Z_{1}} & =\mathbf{r}_{Z_{1}} \\
\mathbf{r}_{Z_{1}+1} & =\mathbf{r}_{Z_{1}}+\mathbf{t}_{Z_{1}} \\
\mathbf{r}_{Z_{1}+2} & =\mathbf{r}_{Z_{1}}+\mathbf{t}_{Z_{1}}+\mathbf{t}_{Z_{1}+1}
\end{aligned}
$$

Now we apply this scheme to the numerator of Eq. (C.5) and first consider the case $Z_{0}>Z_{1}$ :

$$
\begin{aligned}
Z=\frac{1}{A}\left(\frac{2 \pi \Delta z}{\sigma}\right)^{-D\left(Z_{L}-1\right) / 2} \int \mathrm{d} \mathbf{t}_{1} \cdots \mathrm{d} \mathbf{t}_{Z_{1}-1} d \mathbf{r}_{Z_{1}} \mathrm{~d} \mathbf{t}_{Z_{1}} \cdots \mathrm{d} \mathbf{t}_{Z_{L}-1} \\
\quad \exp \left(-\frac{\sigma}{2 \Delta z} \sum_{Z=1}^{Z_{L}-1} \mathbf{t}_{Z}^{2}-\frac{\mathbf{r}_{Z_{1}}^{2}}{2 a^{2}}+\mathbf{b}\left(\mathbf{r}_{Z_{1}}+\mathbf{t}_{Z_{1}}+\ldots+\mathbf{t}_{Z_{0}-1}\right)\right) \\
=\frac{1}{A}\left(\frac{2 \pi \Delta z}{\sigma}\right)^{-D\left(Z_{L}-1\right) / 2} \\
\quad \times\left(\frac{2 \pi \Delta z}{\sigma}\right)^{D\left(Z_{L}-1\right) / 2}\left(2 \pi a^{2}\right)^{D / 2} \exp \left(\frac{\Delta z}{2 \sigma} \mathbf{b}^{2} \cdot\left(Z_{0}-Z_{1}\right)+\frac{\mathbf{b}^{2} a^{2}}{2}\right) \\
=\frac{\left(2 \pi a^{2}\right)^{D / 2}}{A} \exp \left(\frac{\mathbf{b}^{2}}{2 \sigma} \Delta z\left(Z_{0}-Z_{1}\right)+\frac{\mathbf{b}^{2} a^{2}}{2}\right)
\end{aligned}
$$


Analogously we get for $Z_{0}<Z_{1}$ :

$$
Z=\frac{\left(2 \pi a^{2}\right)^{D / 2}}{A} \exp \left(\frac{\mathbf{b}^{2}}{2 \sigma} \Delta z\left(Z_{1}-Z_{0}\right)+\frac{\mathbf{b}^{2} a^{2}}{2}\right),
$$

and thus we get in general:

$$
Z=\frac{\left(2 \pi a^{2}\right)^{D / 2}}{A} \exp \left(\frac{\mathbf{b}^{2}}{2}\left(\frac{\left|z_{1}-z_{0}\right|}{\sigma}+a^{2}\right)\right)
$$

where we re-substituted $z_{i}=Z_{i} \Delta z$ for $i=1,2$. For the logarithm of $Z$ we get:

$$
\ln Z=\frac{\mathbf{b}^{2}}{2}\left(\frac{\left|z_{1}-z_{0}\right|}{\sigma}+a^{2}\right)+\ln \left(\frac{\left(2 \pi a^{2}\right)^{D / 2}}{A}\right) .
$$

\section{C.2 Examples of Single Chain Interactions}

As mentioned in Sec. 3.3.1 on page 39, it is possible to replace the alignment interaction $H_{\text {align }}$ by a more general interaction which also can be written as a sum over individual chains:

$$
H_{\Sigma}=\sum_{j=1}^{N} H_{\Sigma, 1}\left\{\mathbf{r}_{j}\right\}=\sum_{j=1}^{N}\left(H_{\text {align }, 1}\left\{\mathbf{r}_{j}\right\}+H_{X, 1}\left\{\mathbf{r}_{j}\right\}+\ldots\right)
$$

The only requirement is that $H_{\Sigma, 1}\left\{\mathbf{r}_{j}\right\}$ only depends on the conformation $\mathbf{r}_{j}(z)$ of chain $j$. With the total Hamiltonian $H_{\mathcal{C}}=H_{\mathrm{Xlink}}+H_{\mathrm{ev}}+H_{\Sigma}$, the effective one-particle partition function $\mathfrak{z}$, Eq. (3.32c), is simply replaced by:

$$
\begin{aligned}
\mathfrak{z}=\int \mathcal{D} \hat{r}(z) \exp \left(\phi^{n}\right. & \frac{\mu^{2}}{L} \int_{0}^{L} \mathrm{~d} z \sum_{\hat{q} \in \mathrm{HRS}} \Delta(\hat{q}) \Omega(\hat{q}, z) \mathrm{e}^{-\mathrm{i} \hat{q} \hat{r}(z)} \\
& \left.+\mathrm{i} \int_{0}^{L} \mathrm{~d} z \sum_{\hat{q} \in 1 \mathrm{RS}} \tilde{\lambda}(\hat{q}) \Omega(\hat{q}, z) \mathrm{e}^{-\mathrm{i} \hat{q} \hat{r}(z)}-H_{\Sigma, 1}^{(n+1)}\{\hat{r}(z)\}\right),
\end{aligned}
$$

with $H_{\Sigma, 1}^{(n+1)}\{\hat{r}(z)\}=\sum_{\alpha=0}^{n} H_{\Sigma, 1}\left\{\mathbf{r}_{j}^{(\alpha)}\right\}$. The other parts of Eq. (3.32) remain as they are. Beyond the alignment interaction, these interactions $\sum_{j} H_{X, 1}\left\{\mathbf{r}_{j}\right\}$ may include:

- A tilt field $\mathbf{h}(z)$, creating a force on the chains:

$$
H_{\mathrm{tilt}}=\sum_{j=1}^{N} \int_{0}^{L} \mathrm{~d} z \mathbf{h}(z) \cdot \dot{\mathbf{r}}_{j}(z)
$$

For this Hamiltonian, a constant $\mathbf{h}(z)=\mathbf{h}$ corresponds to a linear shear force, since $\mathbf{h}(z)$ couples to the tilt $\dot{\mathbf{r}}_{j}(z)$ of the chains. 
- A pressure field $\Pi(\mathbf{q}, z)$ that couples to the Fourier density:

$$
H_{\Pi}=\sum_{\mathbf{q}} \int_{0}^{L} \mathrm{~d} z \Pi(\mathbf{q}, z) \sum_{j=1}^{N} \mathrm{e}^{-\mathrm{iqr}_{j}(z)}
$$

$$
\begin{array}{ll}
\text { such that } \quad \frac{\delta H_{\Pi}}{\delta \Pi(\mathbf{q}, z)} & =\sum_{j=1}^{N} \mathrm{e}^{-\mathbf{i q} \mathbf{r}_{j}(z)} \\
\text { and } \quad \frac{\delta^{2} H_{\Pi}}{\delta \Pi\left(\mathbf{q}, z_{1}\right) \delta \Pi\left(-\mathbf{q}, z_{2}\right)} & =\sum_{i, j=1}^{N} \mathrm{e}^{-\mathrm{i} \mathbf{q}\left(\mathbf{r}_{i}\left(z_{1}\right)-\mathbf{r}_{j}\left(z_{2}\right)\right)} .
\end{array}
$$

With this field, we would be able to calculate density-density correlations.

- A "clamping interaction", that clamps the ends of the chains, $\mathbf{r}_{j}(0)$ and $\mathbf{r}_{j}(L)$, to certain points at the bottom and top (the boundaries in $z$-direction) of the system:

$$
H_{\text {clamp }}=\frac{1}{2 b_{0}^{2}} \sum_{j=1}^{N}\left(\mathbf{r}_{j}(0)-\mathbf{r}_{0, j}\right)^{2}+\frac{1}{2 b_{L}^{2}} \sum_{j=1}^{N}\left(\mathbf{r}_{j}(L)-\mathbf{r}_{L, j}\right)^{2}
$$

Here $b_{0}$ and $b_{L}$ are the clamping strengths at the bottom $(z=0)$ and the top $(z=L)$ of the system. The clamping points $\mathbf{r}_{0, j}$ and $\mathbf{r}_{L, j}$ could be included in the disorder, $\mathcal{C}=\left(\left\{\left(i_{e}, j_{e}, z_{e}\right)\right\}_{e=1}^{M},\left\{\left(\mathbf{r}_{0, j}, \mathbf{r}_{L, j}\right)\right\}_{j=1}^{N}\right)$, such that their distribution is chosen by the Deam-Edwards distribution. For polymer brushes, which are typically mounted with one end onto a plate, this additional interaction with only one clamping term (i.e. $b_{0}$ finite and $b_{L} \rightarrow \infty$ ) would be an appropriate choice.

- A bending stiffness which penalizes bending, i.e. high values of the second derivative of the chains:

$$
H_{\text {bend }}=\frac{\kappa}{2} \sum_{j=1}^{N} \int_{0}^{L} \mathrm{~d} z \ddot{\mathbf{r}}_{j}^{2}(z)
$$

It is notable that, only this straightening interaction can produce a non-zero persistence length. Without this interaction, the slopes of a given chain at two different points $z_{1}$ and $z_{2}$ are fully uncorrelated, no matter how close those points are. 


\section{C.3 Calculations for the Disorder-Averaged Free En- ergy}

\section{C.3.1 Introduction of Replicas}

In Eq. 3.24, we calculated the important quantity:

$$
\begin{aligned}
& {\left[Z_{\mathcal{C}}^{n}\right]=\frac{1}{\mathcal{Z}_{1}} \sum_{M=0}^{\infty} \frac{\left(\tilde{\mu}^{2} / L\right)^{M}}{M !} \sum_{\substack{i_{1}, \ldots, i_{M}, j_{1}, \ldots, j_{M}=1}}^{N} \int_{0}^{L} \mathrm{~d} z_{1} \cdots \mathrm{d} z_{M}} \\
& \quad \int \mathcal{D} \hat{r}_{1}(z) \cdots \mathcal{D} \hat{r}_{N}(z) \mathrm{e}^{-H_{\mathrm{ev}}^{(n+1)}\left\{\hat{r}_{j}\right\}-H_{\text {align }}^{(n+1)}\left\{\hat{r}_{j}\right\}} \prod_{e=1}^{M} \exp \left(-\frac{\left(\hat{r}_{i_{e}}\left(z_{e}\right)-\hat{r}_{j_{e}}\left(z_{e}\right)\right)^{2}}{2 a^{2}}\right) .
\end{aligned}
$$

Here, we perform the sum over $M$. For simplification, we define:

$$
\mathcal{D} \Gamma:=\mathcal{D} \hat{r}_{1}(z) \cdots \mathcal{D} \hat{r}_{N}(z)
$$

and write the product over $e$ as a power:

$$
\begin{aligned}
{\left[Z_{\mathcal{C}}^{n}\right]=} & \frac{1}{\mathcal{Z}_{1}} \sum_{M=0}^{\infty} \frac{\left(\tilde{\mu}^{2} / L\right)^{M}}{M !} \int \mathcal{D} \Gamma \mathrm{e}^{-H_{\mathrm{ev}}^{(n+1)}\left\{\hat{r}_{j}\right\}-H_{\text {align }}^{(n+1)}\left\{\hat{r}_{j}\right\}} \\
& \left(\sum_{i, j=1}^{N} \int_{0}^{L} \mathrm{~d} z \exp \left(-\frac{\left(\hat{r}_{i}(z)-\hat{r}_{j}(z)\right)^{2}}{2 a^{2}}\right)\right)^{M} \\
& =\frac{1}{\mathcal{Z}_{1}} \int \mathcal{D} \Gamma \mathrm{e}^{-H_{\mathrm{ev}}^{(n+1)}\left\{\hat{r}_{j}\right\}-H_{\text {align }}^{(n+1)}\left\{\hat{r}_{j}\right\}} \\
& \exp \left(\frac{\tilde{\mu}^{2}}{L} \sum_{i, j=1}^{N} \int_{0}^{L} \mathrm{~d} z \exp \left(-\frac{\left(\hat{r}_{i}(z)-\hat{r}_{j}(z)\right)^{2}}{2 a^{2}}\right)\right) \\
= & \frac{\mathcal{Z}_{n+1}}{\mathcal{Z}_{1}} .
\end{aligned}
$$

Here, the replica partition function $\mathcal{Z}_{n+1}$ has been defined:

$$
\mathcal{Z}_{n+1}=\int \mathcal{D} \Gamma \exp \left(-N \tilde{f}_{n+1}\left\{\hat{r}_{j}\right\}\right) \quad \text { with }
$$

with the replica free energy $\tilde{f}_{n+1}\left\{\hat{r}_{j}\right\}$ :

$$
\tilde{f}_{n+1}\left\{\hat{r}_{j}\right\}=\frac{H_{\mathrm{ev}}^{(n+1)}\left\{\hat{r}_{j}\right\}}{N}+\frac{H_{\mathrm{align}}^{(n+1)}\left\{\hat{r}_{j}\right\}}{N}-\frac{\tilde{\mu}^{2}}{L N} \sum_{i, j=1}^{N} \int_{0}^{L} \mathrm{~d} z \exp \left(-\frac{\left(\hat{r}_{i}(z)-\hat{r}_{j}(z)\right)^{2}}{2 a^{2}}\right)
$$




\section{C.3.2 Introduction of the Replicated Density Field}

In Sec. 3.4.2, we introduced the replicated density field, Eqs. (3.29). Here we rewrite the replica free energy $(3.27 \mathrm{c})$,

$$
\begin{aligned}
\tilde{f}_{n+1}\left\{\hat{r}_{j}\right\}= & -\frac{\tilde{\mu}^{2}}{L N} \sum_{i, j=1}^{N} \int_{0}^{L} \mathrm{~d} z \exp \left(-\frac{\left(\hat{r}_{i}(z)-\hat{r}_{j}(z)\right)^{2}}{2 a^{2}}\right) \\
& +\frac{\lambda}{2 N} \sum_{\alpha=0}^{n} \sum_{i, j=1}^{N} \int_{0}^{L} \mathrm{~d} z U\left(\mathbf{r}_{i}^{(\alpha)}(z)-\mathbf{r}_{j}^{(\alpha)}(z)\right)+\frac{H_{\text {align }}^{(n+1)}}{N},
\end{aligned}
$$

in terms of that field. In exactly the same manner as in Eqs. (B.5-B.7), we can rewrite the cross-linking term as:

$$
\sum_{i, j=1}^{N} \exp \left(-\frac{\left(\hat{r}_{i}(z)-\hat{r}_{j}(z)\right)^{2}}{2 a^{2}}\right)=\phi^{n+1} N^{2} \sum_{\hat{q}}|\mathrm{O}(\hat{q}, z)|^{2} \Delta(\hat{q})
$$

with

$$
\Delta(\hat{q})=\exp \left(-\frac{\hat{q}^{2} a^{2}}{2}\right) .
$$

For the second (excluded volume) term in Eq. (C.21), we first define the density field for each replica separately:

$$
\begin{aligned}
& \mathrm{O}^{(\alpha)}(\mathbf{x}, z)=\frac{1}{N} \sum_{j=1}^{N} \delta\left(\mathbf{x}-\mathbf{r}_{j}^{(\alpha)}(z)\right) \\
& \mathrm{O}^{(\alpha)}(\mathbf{q}, z)=\frac{1}{N} \sum_{j=1}^{N} \exp \left(\mathrm{iqr}_{j}^{(\alpha)}(z)\right) .
\end{aligned}
$$

With that, also the excluded volume Hamiltonian can be rewritten in analogy to the RLP model, see Eq. (B.9), we just have to add the integral over $z$ :

$$
\begin{gathered}
\sum_{\alpha=0}^{n} \sum_{i, j=1}^{N} \int_{0}^{L} \mathrm{~d} z U\left(\mathbf{r}_{i}^{(\alpha)}(z)-\mathbf{r}_{j}^{(\alpha)}(z)\right) \\
=\frac{N^{2}}{A} \sum_{\alpha=0}^{n} \sum_{\mathbf{q}^{(\alpha)}} \int_{0}^{L} \mathrm{~d} z\left|\mathrm{O}^{(\alpha)}\left(\mathbf{q}^{(\alpha)}, z\right)\right|^{2} U\left(\mathbf{q}^{(\alpha)}\right) .
\end{gathered}
$$

With that and by plugging in $\frac{\tilde{\mu}^{2}}{L N}=\frac{\mu^{2}}{2 L N^{2} \phi}$ (see Eq. $(3.22 \mathrm{c})$ ), we can write the replica free energy, Eq. (C.21), as:

$$
\begin{aligned}
\tilde{f}_{n+1}\left\{\hat{r}_{j}\right\}= & -\phi^{n} \frac{\mu^{2}}{2 L} \int_{0}^{L} \mathrm{~d} z \sum_{\hat{q}}|\mathrm{O}(\hat{q}, z)|^{2} \Delta(\hat{q}) \\
& +\frac{\lambda n_{0}}{2} \int_{0}^{L} \mathrm{~d} z \sum_{\alpha=0}^{n} \sum_{\mathbf{q}^{(\alpha)}}\left|\mathrm{O}^{(\alpha)}\left(\mathbf{q}^{(\alpha)}, z\right)\right|^{2} U\left(\mathbf{q}^{(\alpha)}\right)+\frac{H_{\text {align }}^{(n+1)}\left\{\hat{r}_{j}\right\}}{N},
\end{aligned}
$$


where we defined the mean chain density

$$
n_{0}=\frac{N}{A}
$$

as number of chains per (hyper-)area.

We now separate the two terms involving fields in Eq. (C.26) into one replica sector (1RS), the set of replicated $\hat{q}$-vectors with exactly one of the $n+1$ components non-zero, and higher replica sector (HRS), the set of replicated $\hat{q}$-vectors with at least two non-zero components, as introduced in Sec. 2.4.3. This will allow us to perform the Hubbard-Stratonovich transformation later.

$$
\begin{aligned}
\tilde{f}_{n+1}\left\{\hat{r}_{j}\right\}= & \overbrace{-\phi^{n} \frac{\mu^{2}}{2}+(n+1) \frac{\lambda n_{0} L}{2}}^{=: f_{0}}-\phi^{n} \frac{\mu^{2}}{2 L} \int_{0}^{L} \mathrm{~d} z \sum_{\hat{q} \in \mathrm{HRS}}|\mathrm{O}(\hat{q}, z)|^{2} \Delta(\hat{q}) \\
& +\frac{1}{2} \int_{0}^{L} \mathrm{~d} z \sum_{\hat{q} \in 1 \mathrm{RS}} \underbrace{\left(\lambda n_{0} U(\hat{q})-\phi^{n} \frac{\mu^{2}}{L} \Delta(\hat{q})\right)}_{=: \tilde{\lambda}(\hat{q})}|\mathrm{O}(\hat{q}, z)|^{2}+\frac{H_{\text {align }}^{(n+1)}\left\{\hat{r}_{j}\right\}}{N}
\end{aligned}
$$

For the terms involving $\hat{q}=\hat{0}$, which are neither part of the 1RS, nor of the HRS, we used $\mathrm{O}(\hat{q}=\hat{0})=1, \Delta(\hat{q}=\hat{0})=1$ and $U(\mathbf{q}=\mathbf{0})=1$.

\section{C.3.3 The Hubbard-Stratonovich Transformation}

Here we want to apply the Hubbard-Stratonovich transformation to the replica free energy Eq. (3.31a) to linearize it with respect to the field $\mathrm{O}(\hat{q}, z)$. The appropriate variable for the transformation is $\mathrm{O}(\hat{q}, z)$, similar to the RLP model. A transformation with respect to $\int \mathrm{d} z \mathrm{O}(\hat{q}, z)$, similar to what has been done in [Goldbart et al., 1996, Chapter V], would require a different structure, a form like $\int \mathrm{d} z_{1} \mathrm{~d} z_{2} \mathrm{O}\left(\hat{q}, z_{1}\right) \mathrm{O}\left(-\hat{q}, z_{2}\right)$ in the replica free energy. Hence, we apply Eqs. (B.12) successively for all combinations of $\hat{q}$ and $z$.

Before performing the transformation, it is important to note that the replica density obeys the relation $\mathrm{O}(-\hat{q}, z)=\mathrm{O}^{*}(\hat{q}, z)$ for any height $z$, see definition (3.29b), in particular $|\mathrm{O}(\hat{q}, z)|^{2}=|\mathrm{O}(-\hat{q}, z)|^{2}$; hence, for any $\hat{q}$, we automatically transform the $|\mathrm{O}(\hat{q}, z)|^{2}$-term along the $|\mathrm{O}(-\hat{q}, z)|^{2}$-term. In order to carry out the Hubbard-Stratonovich transformation, we restrict the sums over $\hat{q}$ to a half-space with $\hat{q} \cdot \hat{e}>0$ with $\hat{e}=(\mathbf{e}, \ldots, \mathbf{e})$ for arbitrary $\mathbf{e} \neq \mathbf{0}$ (as it was done in Sec. B.2.1 for the RLP model). The terms for $\hat{q}$ and $-\hat{q}$ yield the same contribution, since $\Delta(\hat{q})=\Delta(-\hat{q})$ and $\tilde{\lambda}(\hat{q})=\tilde{\lambda}(-\hat{q})$. 
With that we get for replica partition function $\mathcal{Z}_{n+1}$, Eq. (3.27b):

$$
\begin{aligned}
& \frac{\mathcal{Z}_{n+1}}{\exp \left(-N f_{0}\right)}=\int\left(\prod_{j=1}^{N} \mathcal{D} \hat{r}_{j}(z)\right) \\
& \quad \exp \left(N \phi^{n} \frac{\mu^{2}}{L} \int_{0}^{L} \mathrm{~d} z \sum_{\substack{\hat{q} \in \operatorname{HRS} \\
\hat{q} \cdot \hat{e}>0}}|\mathrm{O}(\hat{q}, z)|^{2} \Delta(\hat{q})-N \int_{0}^{L} \mathrm{~d} z \sum_{\substack{\hat{q} \in 1 \mathrm{RS} \\
\hat{q} \cdot \hat{e}>0}}|\mathrm{O}(\hat{q}, z)|^{2} \tilde{\lambda}(\hat{q})-H_{\text {align }}^{(n+1)}\right)
\end{aligned}
$$

Now we apply the Hubbard-Stratonovich transformation with $w=\mathrm{O}(\hat{q}, z)$ : For any $\hat{q} \in 1 \mathrm{RS}, \hat{q} \cdot \hat{e}>0$ and any $z \in[0, L]$ we use Eq. (B.12b) with $c=N \tilde{\lambda}(\hat{q})$ :

$$
\begin{aligned}
& \exp \left(-N|\mathrm{O}(\hat{q}, z)|^{2} \tilde{\lambda}(\hat{q})\right) \\
& =\int \frac{N \tilde{\lambda}(\hat{q}) \mathrm{d} \Omega(\hat{q}, z)}{\pi} \exp \left(-N|\Omega(\hat{q}, z)|^{2} \tilde{\lambda}(\hat{q})+2 \mathrm{i} N \tilde{\lambda}(\hat{q}) \operatorname{Re}(\Omega(\hat{q}, z) \mathrm{O}(-\hat{q}, z))\right)
\end{aligned}
$$

and for $\hat{q} \in \operatorname{HRS}, \hat{q} \cdot \hat{e}>0$ and $z \in[0, L]$ we use Eq. (B.12a) with $c=N \phi^{n} \frac{\mu^{2}}{L} \Delta(\hat{q})$ :

$$
\begin{gathered}
\exp \left(N \phi^{n} \frac{\mu^{2}}{L}|\mathrm{O}(\hat{q}, z)|^{2} \Delta(\hat{q})\right)=\int \frac{N \phi^{n} \mu^{2} \Delta(\hat{q}) \mathrm{d} \Omega(\hat{q}, z)}{\pi L} \\
\exp \left(-N \phi^{n} \frac{\mu^{2}}{L} \Delta(\hat{q})|\Omega(\hat{q}, z)|^{2}+2 N \phi^{n} \frac{\mu^{2}}{L} \Delta(\hat{q}) \operatorname{Re}\left(\Omega(\hat{q}, z) \mathrm{O}\left(-q^{\hat{,} z} z\right)\right)\right)
\end{gathered}
$$

With that Eq. (C.29) becomes:

$$
\begin{aligned}
& \frac{\mathcal{Z}_{n+1}}{\exp \left(-N f_{0}\right)}= \\
& \int \mathcal{D} \Omega \exp \left(-N \phi^{n} \frac{\mu^{2}}{L} \int_{0}^{L} \mathrm{~d} z \sum_{\substack{\hat{q} \in \operatorname{HRS} \\
\hat{q} \cdot \hat{e}>0}}|\Omega(\hat{q}, z)|^{2} \Delta(\hat{q})-N \int_{0}^{L} \mathrm{~d} z \sum_{\substack{\hat{q} \in 1 \mathrm{RS} \\
\hat{q} \cdot \hat{e}>0}}|\Omega(\hat{q}, z)|^{2} \tilde{\lambda}(\hat{q})\right) \\
& \times \int\left(\prod_{j=1}^{N} \mathcal{D} \hat{r}_{j}(z)\right) \exp \left(2 N \phi^{n} \frac{\mu^{2}}{L} \int_{0}^{L} \mathrm{~d} z \sum_{\substack{\hat{q} \in \operatorname{HRS} \\
\hat{q} \cdot \hat{e}>0}} \Delta(\hat{q}) \operatorname{Re}(\Omega(\hat{q}, z) \mathrm{O}(-\hat{q}, z))\right. \\
& \left.+2 \mathrm{i} N \int_{0}^{L} \mathrm{~d} z \sum_{\substack{\hat{q} \in 1 \mathrm{RS} \\
\hat{q} \cdot \hat{e}>0}} \tilde{\lambda}(\hat{q}) \operatorname{Re}(\Omega(\hat{q}, z) \mathrm{O}(-\hat{q}, z))-H_{\text {align }}^{(n+1)}\left(\left\{\hat{r}_{j}\right\}\right)\right),
\end{aligned}
$$

where we introduced the measure

$$
\mathcal{D} \Omega=\prod_{z}\left(\prod_{\substack{\hat{q} \in 1 \mathrm{RS} \\ \hat{q} \cdot \hat{e}>0}} \frac{N \tilde{\lambda}(\hat{q}) \mathrm{d} \Omega(\hat{q}, z)}{\pi} \cdot \prod_{\substack{\hat{q} \in \mathrm{HRS} \\ \hat{q} \cdot \hat{e}>0}} \frac{N \phi^{n} \mu^{2} \Delta(\hat{q}) \mathrm{d} \Omega(\hat{q}, z)}{\pi L}\right) .
$$

Here, the product over $z$ has to be carried out in the spirit of the discretization of the $z$-direction into $Z_{L}$ segments, as seen in Sec. 3.3 and Fig. 3.5. It implies that we have to integrate over $\Omega(\hat{q}, z)$ for all $\hat{q}$ and at every height $z$. 
Now we abolish the constraint $\hat{q} \cdot \hat{e}>0$ in the sums. Therefore define the field $\Omega(\hat{q}, z)$ also for $\hat{q} \cdot \hat{e}<0$ by

$$
\Omega(-\hat{q}, z)=\Omega^{*}(\hat{q}, z) .
$$

and plug in the definition of $\mathrm{O}(\hat{q}, z)$, Eq. (3.29b). Furthermore we recall that $H_{\text {align }}^{(n+1)}$ acts on all chains in the same way (see Eqs. (3.17), (3.19)):

$$
H_{\text {align }}^{(n+1)}=\sum_{j=1}^{N} H_{\text {align }, 1}^{(n+1)}\left\{\hat{r}_{j}\right\}
$$

Hence the partition function (C.32) becomes:

$$
\begin{aligned}
& \frac{\mathcal{Z}_{n+1}}{\exp \left(-N f_{0}\right)}= \\
& \int \mathcal{D} \Omega \exp \left(-N \phi^{n} \frac{\mu^{2}}{2 L} \int_{0}^{L} \mathrm{~d} z \sum_{\hat{q} \in \mathrm{HRS}}|\Omega(\hat{q}, z)|^{2} \Delta(\hat{q})-\frac{N}{2} \int_{0}^{L} \mathrm{~d} z \sum_{\hat{q} \in 1 \mathrm{RS}}|\Omega(\hat{q}, z)|^{2} \tilde{\lambda}(\hat{q})\right) \\
& \times \int\left(\prod_{j=1}^{N} \mathcal{D} \hat{r}_{j}(z)\right) \exp \left(\phi^{n} \frac{\mu^{2}}{L} \sum_{j=1}^{N} \int_{0}^{L} \mathrm{~d} z \sum_{\hat{q} \in \mathrm{HRS}} \Delta(\hat{q}) \Omega(\hat{q}, z) \mathrm{e}^{-\mathrm{i} \hat{q}_{j}(z)}\right. \\
& \left.+\mathrm{i} \sum_{j=1}^{N} \int_{0}^{L} \mathrm{~d} z \sum_{\hat{q} \in 1 \mathrm{RS}} \tilde{\lambda}(\hat{q}) \Omega(\hat{q}, z) \mathrm{e}^{-\mathrm{i} \hat{q} \hat{r}_{j}(z)}-\sum_{j=1}^{N} H_{\text {align, } 1}^{(n+1)}\left\{\hat{r}_{j}(z)\right\}\right)
\end{aligned}
$$

As in the RLP model before (see Sec. 2.4.4 and B.2.1), the expression is now linear in the density and therefore only generates "linear" sums $\sum_{j}$ over all particles. We also see, why the term $H_{\text {align }}^{(n+1)}$ was so easy to take along and did not require a Hubbard-Stratonovich transformation: it is already linear in the sum $\sum_{j}$. Now, the sums in the exponential and the difficult integral over all chains can be written as a power:

$$
\begin{aligned}
& \frac{\mathcal{Z}_{n+1}}{\exp \left(-N f_{0}\right)}= \\
& \int \mathcal{D} \Omega \exp \left(-N \phi^{n} \frac{\mu^{2}}{2 L} \int_{0}^{L} \mathrm{~d} z \sum_{\hat{q} \in \mathrm{HRS}}|\Omega(\hat{q}, z)|^{2} \Delta(\hat{q})-\frac{N}{2} \int_{0}^{L} \mathrm{~d} z \sum_{\hat{q} \in 1 \mathrm{RS}}|\Omega(\hat{q}, z)|^{2} \tilde{\lambda}(\hat{q})\right) \\
& \times\left\{\int \mathcal { D } \hat { r } ( z ) \operatorname { e x p } \left(\phi^{n} \frac{\mu^{2}}{L} \int_{0}^{L} \mathrm{~d} z \sum_{\hat{q} \in \mathrm{HRS}} \Delta(\hat{q}) \Omega(\hat{q}, z) \mathrm{e}^{-\mathrm{i} \hat{q} \hat{r}(z)}\right.\right. \\
& \left.\left.+\mathrm{i} \int_{0}^{L} \mathrm{~d} z \sum_{\hat{q} \in 1 \mathrm{RS}} \tilde{\lambda}(\hat{q}) \Omega(\hat{q}, z) \mathrm{e}^{-\mathrm{i} \hat{q} \hat{r}(z)}-H_{\text {align, },(n+1)}^{(n)}\{\hat{r}(z)\}\right)\right\}^{N}
\end{aligned}
$$

And that form can be written in a simplified way:

$$
\mathcal{Z}_{n+1}=\exp \left(-N f_{0}\right) \int \mathcal{D} \Omega \exp \left(-N f_{n+1}\{\Omega\}\right)
$$


with the replica free energy

$$
f_{n+1}\{\Omega\}=\phi^{n} \frac{\mu^{2}}{2 L} \int_{0}^{L} \mathrm{~d} z \sum_{\hat{q} \in \mathrm{HRS}}|\Omega(\hat{q}, z)|^{2} \Delta(\hat{q})+\frac{1}{2} \int_{0}^{L} \mathrm{~d} z \sum_{\hat{q} \in 1 \mathrm{RS}}|\Omega(\hat{q}, z)|^{2} \tilde{\lambda}(\hat{q})-\ln \mathfrak{z},
$$

and an effective one-particle partition function

$$
\begin{aligned}
\mathfrak{z}=\int \mathcal{D} \hat{r}(z) \exp \left(\phi^{n}\right. & \frac{\mu^{2}}{L} \int_{0}^{L} \mathrm{~d} z \sum_{\hat{q} \in \mathrm{HRS}} \Delta(\hat{q}) \Omega(\hat{q}, z) \mathrm{e}^{-\mathrm{i} \hat{q} \hat{r}(z)} \\
& \left.+\mathrm{i} \int_{0}^{L} \mathrm{~d} z \sum_{\hat{q} \in 1 \mathrm{RS}} \tilde{\lambda}(\hat{q}) \Omega(\hat{q}, z) \mathrm{e}^{-\mathrm{i} \hat{q} \hat{r}(z)}-H_{\text {align, } 1}^{(n+1)}\{\hat{r}(z)\}\right)
\end{aligned}
$$

\section{C.4 The Saddle-Point Equation with Ansatz}

\section{C.4.1 Expansion in $Q$ to Infinite Order}

Here we calculate the term $I\left(\hat{q}_{0}, z_{0}\right)$, Eq. (3.50). With the single chain alignment interaction:

$$
H_{\text {align,1 }}^{(n+1)}\{\hat{r}(z)\} \equiv \frac{\sigma}{2} \int_{0}^{L} \mathrm{~d} z \dot{\hat{r}}^{2}(z)
$$

we get:

$$
\begin{aligned}
& I\left(\hat{q}_{0}, z_{0}\right)=\int \mathcal{D} \hat{r}(z) \mathrm{e}^{-\mathrm{i} \hat{q}_{0} \hat{r}\left(z_{0}\right)} \\
& \times \exp \left(\phi^{n} \frac{\mu^{2} Q}{L} \int_{0}^{L} \mathrm{~d} z \sum_{\hat{q}} \mathrm{e}^{-\mathrm{i} \hat{q} \hat{r}(z)} \delta_{\mathbf{q}_{\|}, \mathbf{0}} \int_{\xi^{2}, z} \exp \left(-\frac{\hat{q}^{2} \tilde{\xi}^{2}}{2}\right)-\frac{\sigma}{2} \int_{0}^{L} \mathrm{~d} z \dot{\hat{r}}^{2}(z)\right) .
\end{aligned}
$$

To proceed in the calculation, we would like to perform the functional integral over all chain positions $\hat{r}(z)$. Therefore we expand the integrand of $I\left(\hat{q}_{0}, z_{0}\right)$, Eq. (C.40), in powers of $Q$ :

$$
\begin{gathered}
I\left(\hat{q}_{0}, z_{0}\right)=\int \mathcal{D} \hat{r}(z) \mathrm{e}^{-\mathrm{i} \hat{q}_{0} \hat{r}\left(z_{0}\right)} \exp \left(-\frac{\sigma}{2} \int_{0}^{L} \mathrm{~d} z \dot{\hat{r}}^{2}(z)\right) \\
\times\left(1+\sum_{r=1}^{\infty} \frac{\left(\phi^{n} \mu^{2} Q\right)^{r}}{r !}\left\{\frac{1}{L} \int_{0}^{L} \mathrm{~d} z \sum_{\hat{q}} \mathrm{e}^{-\mathrm{i} \hat{q} \hat{r}(z)} \delta_{\mathbf{q}_{\|}, \mathbf{0}} \int_{\xi^{2}, z} \exp \left(-\frac{\hat{q}^{2} \tilde{\xi}^{2}}{2}\right)\right\}\right) \\
=\int \mathcal{D} \hat{r}(z) \mathrm{e}^{-\mathrm{i} \hat{q}_{0} \hat{r}\left(z_{0}\right)} \exp \left(-\frac{\sigma}{2} \int_{0}^{L} \mathrm{~d} z \dot{\hat{r}}^{2}(z)\right) \\
\times\left(1+\sum_{r=1}^{\infty} \frac{\left(\phi^{n} \mu^{2} Q\right)^{r}}{r ! L^{r}} \int_{0}^{L} \mathrm{~d} z_{1} \cdots \mathrm{d} z_{r} \sum_{\hat{q}_{1}, \ldots, \hat{q}_{r}} \delta_{\mathbf{q}_{1 \|}, \mathbf{0}} \cdots \delta_{\mathbf{q}_{r \|}, \mathbf{0}} \mathrm{e}^{-\mathrm{i}\left(\hat{q}_{1} \hat{r}\left(z_{1}\right)+\cdots+\hat{q}_{r} \hat{r}\left(z_{r}\right)\right)}\right. \\
\left.\times \int_{\xi_{1}^{2}, z_{1}} \ldots \int_{\xi_{r}^{2}, z_{r}} \exp \left(-\frac{\hat{q}_{1}^{2} \tilde{\xi}_{1}^{2}+\ldots+\hat{q}_{r}^{2} \tilde{\xi}_{r}^{2}}{2}\right)\right)
\end{gathered}
$$


All $\hat{r}(z)$-dependent terms, which are needed to perform the functional integral, are marked in blue. This integral is calculated in Appendix C.6 and the result is given by Eq. (C.88):

$$
\begin{aligned}
& \int \mathcal{D} \hat{r}(z) \mathrm{e}^{-\mathrm{i}\left(\hat{q}_{0} \hat{r}\left(z_{0}\right)+\hat{q}_{1} \hat{r}\left(z_{1}\right)+\ldots+\hat{q}_{r} \hat{r}\left(z_{r}\right)\right)} \exp \left(-\frac{\sigma}{2} \int_{0}^{L} \mathrm{~d} z \dot{\hat{r}}^{2}(z)\right) \\
= & \delta_{\hat{q}_{0}+\hat{q}_{1}+\ldots+\hat{q}_{r}, \hat{0}} \exp \left(\frac{1}{2 \sigma} \sum_{0 \leq \gamma<\delta \leq r} \hat{q}_{\gamma} \hat{q}_{\delta}\left|z_{\gamma}-z_{\delta}\right|\right),
\end{aligned}
$$

and therefore:

$$
\begin{aligned}
& I\left(\hat{q}_{0}, z_{0}\right)=\delta_{\hat{q}_{0}, \hat{0}}+\sum_{r=1}^{\infty} \frac{\left(\phi^{n} \mu^{2} Q\right)^{r}}{r ! L^{r}} \int_{0}^{L} \mathrm{~d} z_{1} \cdots \mathrm{d} z_{r} \sum_{\hat{q}_{1}, \ldots, \hat{q}_{r}} \delta_{\mathbf{q}_{1 \|}, \mathbf{0}} \cdots \delta_{\mathbf{q}_{r \|}, \mathbf{0}} \delta_{\hat{q}_{0}+\hat{q}_{1}+\ldots+\hat{q}_{r}, \hat{0}} \\
& \quad \times \exp \left(\frac{1}{2 \sigma} \sum_{0 \leq \gamma<\delta \leq r} \hat{q}_{\gamma} \hat{q}_{\delta}\left|z_{\gamma}-z_{\delta}\right|\right) \int_{\xi_{1}^{2}, z_{1}} \cdots \int_{\xi_{r}^{2}, z_{r}} \exp \left(-\frac{\hat{q}_{1}^{2} \tilde{\xi}_{1}^{2}+\ldots+\hat{q}_{r}^{2} \tilde{\xi}_{r}^{2}}{2}\right)
\end{aligned}
$$

For this expression it is important to keep in mind that the sum $\sum_{0 \leq \gamma<\delta \leq r}$ in the first exponential also involves the external variables $\hat{q}_{0}$ and $z_{0}$. We also see at this point that it is useful to normalize the height $z$ by $L$ to make it a dimensionless variable

$$
s_{\gamma}:=\frac{z_{\gamma}}{L} \in[0,1] \quad \text { and hence } \quad \mathrm{d} s_{\gamma}=\frac{\mathrm{d} z_{\gamma}}{L}
$$

for $\gamma=0, \ldots, r$ in all orders. As already mentioned in Sec. 3.2.1 it is useful to define

$$
\ell^{2}:=\frac{L}{2 \sigma},
$$

which essentially is the radius of gyration of the chain, projected along the $z$-axis. With that we get:

$$
\frac{1}{2 \sigma} \hat{q}_{\gamma} \hat{q}_{\delta}\left|z_{\gamma}-z_{\delta}\right|=\frac{L}{2 \sigma} \hat{q}_{\gamma} \hat{q}_{\delta}\left|s_{\gamma}-s_{\delta}\right|=\ell^{2} \hat{q}_{\gamma} \hat{q}_{\delta}\left|s_{\gamma}-s_{\delta}\right|
$$

Therefore:

$$
\begin{aligned}
I\left(\hat{q}_{0}, s_{0}\right)= & \\
=\delta_{\hat{q}_{0}, \hat{0}} & +\sum_{r=1}^{\infty} \frac{\left(\phi^{n} \mu^{2} Q\right)^{r}}{r !} \int_{0}^{1} \mathrm{~d} s_{1} \cdots \mathrm{d} s_{r} \sum_{\hat{q}_{1}, \ldots, \hat{q}_{r}} \delta_{\mathbf{q}_{1 \|}, \mathbf{0}} \cdots \delta_{\mathbf{q}_{r \|}, \mathbf{0}} \delta_{\hat{q}_{0}+\hat{q}_{1}+\ldots+\hat{q}_{r}, \hat{0}} \\
& \times \exp \left(\ell^{2} \sum_{0 \leq \gamma<\delta \leq r} \hat{q}_{\gamma} \hat{q}_{\delta}\left|s_{\gamma}-s_{\delta}\right|\right) \int_{\xi_{1}^{2}, s_{1}} \cdots \int_{\xi_{r}^{2}, s_{r}} \exp \left(-\frac{1}{2} \sum_{\gamma=1}^{r} \hat{q}_{\gamma}^{2} \tilde{\xi}_{\gamma}^{2}\right) .
\end{aligned}
$$


Here we needed to define the integral

$$
\int_{\xi^{2}, s}:=\int_{0}^{\infty} \mathrm{d} \xi^{2} \mathcal{P}\left(\xi^{2}, s\right)
$$

where $\mathcal{P}\left(\xi^{2}, s\right)$ depends on the dimensionless height $s$, but apart from that it is the same as $\mathcal{P}\left(\xi^{2}, z\right)$. Furthermore, as we can see in Eq. (C.47), the only $L$-dependence remains in the prefactor $\ell^{2}$ in the first exponential.

\section{C.4.2 Expansion to Second Order in $Q$}

Up to this point it was easy to keep $I\left(\hat{q}_{0}, s_{0}\right)$ up to infinite order in $Q$. In order to proceed in the calculation, we keep the expansion up to second order (thereby restricting the system to being close to the sol-gel transition) and separate the different orders:

$$
\begin{aligned}
& I\left(\hat{q}_{0}, s_{0}\right)=\delta_{\hat{q}_{0}, \hat{0}}+ \\
& +\phi^{n} \mu^{2} Q \int_{0}^{1} \mathrm{~d} s_{1} \sum_{\hat{q}_{1}} \delta_{\mathbf{q}_{1 \|}, \mathbf{0}} \delta_{\hat{q}_{0}+\hat{q}_{1}, \hat{0}} \exp \left(\ell^{2} \hat{q}_{0} \hat{q}_{1}\left|s_{0}-s_{1}\right|\right) \int_{\xi_{1}^{2}, s_{1}} \exp \left(-\frac{\hat{q}_{1}^{2} \tilde{\xi}_{1}^{2}}{2}\right) \\
& +\frac{\left(\phi^{n} \mu^{2} Q\right)^{2}}{2} \int_{0}^{1} \mathrm{~d} s_{1} \mathrm{~d} s_{2} \sum_{\hat{q}_{1}, \hat{q}_{2}} \delta_{\mathbf{q}_{1 \|}, \mathbf{0}} \delta_{\mathbf{q}_{2 \|}, \mathbf{0}} \delta_{\hat{q}_{0}+\hat{q}_{1}+\hat{q}_{2}, \hat{0}} \\
& \quad \times \exp \left(\ell^{2}\left(\hat{q}_{0} \hat{q}_{1}\left|s_{0}-s_{1}\right|+\hat{q}_{0} \hat{q}_{2}\left|s_{0}-s_{2}\right|+\hat{q}_{1} \hat{q}_{2}\left|s_{1}-s_{2}\right|\right)\right) \\
& \quad \times \int_{\xi_{1}^{2}, s_{1}} \int_{\xi_{2}^{2}, s_{2}} \exp \left(-\frac{\hat{q}_{1}^{2} \tilde{\xi}_{1}^{2}+\hat{q}_{2}^{2} \tilde{\xi}_{2}^{2}}{2}\right) \\
& +\mathcal{O}\left(Q^{3}\right) .
\end{aligned}
$$

In the first order term, the sum over $\hat{q}_{1}$ is easy to perform thanks to the $\delta_{\hat{q}_{0}+\hat{q}_{1}, \hat{0}^{-}}$ constraint:

$$
\begin{aligned}
I_{1} & :=\sum_{\hat{q}_{1}} \delta_{\mathbf{q}_{1 \|}, \mathbf{0}} \delta_{\hat{q}_{0}+\hat{q}_{1}, \hat{0}} \exp \left(\ell^{2} \hat{q}_{0} \hat{q}_{1}\left|s_{0}-s_{1}\right|\right) \exp \left(-\frac{\hat{q}_{1}^{2} \tilde{\xi}_{1}^{2}}{2}\right) \\
& =\delta_{\mathbf{q}_{0 \|}, \mathbf{0}} \exp \left(-\ell^{2} \hat{q}_{0}^{2}\left|s_{0}-s_{1}\right|-\frac{\hat{q}_{0}^{2} \tilde{\xi}_{1}^{2}}{2}\right)=\delta_{\mathbf{q}_{0 \|}, \mathbf{0}} \exp \left(-\frac{q_{0 \perp}^{2}}{2}\left(2 \ell^{2}\left|s_{0}-s_{1}\right|+\tilde{\xi}_{1}^{2}\right)\right)
\end{aligned}
$$

In the last line we used the fact that $\hat{q}_{0}=q_{0 \perp}$, since $\mathbf{q}_{0 \|}=\mathbf{0}$.

In the second order term, the situation is a little bit more difficult. First get rid of $\hat{q}_{2}$ in the same manner:

$$
\begin{aligned}
I_{2}:= & \sum_{\hat{q}_{1}, \hat{q}_{2}} \delta_{\mathbf{q}_{1 \|},}, \mathbf{0} \delta_{\mathbf{q}_{2 \|},}, \mathbf{0} \\
& \delta_{\hat{q}_{0}+\hat{q}_{1}+\hat{q}_{2}, \hat{0}} \\
& \quad \times \exp \left(\ell^{2}\left(\hat{q}_{0} \hat{q}_{1}\left|s_{0}-s_{1}\right|+\hat{q}_{0} \hat{q}_{2}\left|s_{0}-s_{2}\right|+\hat{q}_{1} \hat{q}_{2}\left|s_{1}-s_{2}\right|\right)\right) \exp \left(-\frac{\hat{q}_{1}^{2} \tilde{\xi}_{1}^{2}+\hat{q}_{2}^{2} \tilde{\xi}_{2}^{2}}{2}\right) \\
= & \sum_{\hat{q}_{1}} \delta_{\mathbf{q}_{0 \|},}, \mathbf{0} \delta_{\mathbf{q}_{1 \|}, \mathbf{0}}
\end{aligned}
$$




$$
\begin{aligned}
& \times \exp \left(\ell^{2}\left(\hat{q}_{0} \hat{q}_{1}\left|s_{0}-s_{1}\right|-\hat{q}_{0}\left(\hat{q}_{0}+\hat{q}_{1}\right)\left|s_{0}-s_{2}\right|-\hat{q}_{1}\left(\hat{q}_{0}+\hat{q}_{1}\right)\left|s_{1}-s_{2}\right|\right)\right) \\
& \times \exp \left(-\frac{\hat{q}_{1}^{2} \tilde{\xi}_{1}^{2}+\left(\hat{q}_{0}+\hat{q}_{1}\right)^{2} \tilde{\xi}_{2}^{2}}{2}\right) \\
=\delta_{\mathbf{q}_{0 \|}, \mathbf{0}} & \exp \left(-\hat{q}_{0}^{2} \ell^{2}\left|s_{0}-s_{2}\right|-\frac{\hat{q}_{0}^{2} \tilde{\xi}_{2}^{2}}{2}\right) \sum_{\hat{q}_{1}} \delta_{\mathbf{q}_{1 \|}, \mathbf{0}} \exp \left(-\hat{q}_{1}^{2}\left(\ell^{2}\left|s_{1}-s_{2}\right|+\frac{\tilde{\xi}_{1}^{2}+\tilde{\xi}_{2}^{2}}{2}\right)\right) \\
& \times \exp \left(\hat{q}_{0} \hat{q}_{1}\left(\ell^{2}\left(\left|s_{0}-s_{1}\right|-\left|s_{0}-s_{2}\right|-\left|s_{1}-s_{2}\right|\right)-\tilde{\xi}_{2}^{2}\right)\right)
\end{aligned}
$$

Now let us look at the sum $\sum_{\hat{q}_{1}}$ : it runs over elements $\hat{q}_{1}=\frac{2 \pi}{A^{1 / D}} \hat{n}$ with $\hat{n} \in \mathbb{Z}^{D(n+1)}$. As already noted in Sec. B.5.3 on page 138, it approaches a Riemann sum in the thermodynamic limit $A \rightarrow \infty$. There we changed

$$
\sum_{\hat{q}_{1}}(\ldots) \longrightarrow \frac{A^{n+1}}{(2 \pi)^{D(n+1)}} \int \mathrm{d} \hat{q}(\ldots)
$$

Here, we additionally have to deal with the $\delta_{\mathbf{q}_{1 \|}, \mathbf{0}^{-}}$-constraint. To include it into the transformation, we can write it as an integral over an exponential. For any function $\mathcal{A}(\hat{q})$ :

$$
\begin{aligned}
& \sum_{\hat{q}} \delta_{\mathbf{q}_{\|}, \mathbf{0}} \mathcal{A}(\hat{q})=\frac{1}{A} \int_{A} \mathrm{~d} \mathbf{x} \sum_{\hat{q}} \mathcal{A}(\hat{q}) \mathrm{e}^{\mathrm{i}\left(\mathbf{q}^{(0)}+\ldots+\mathbf{q}^{(n)}\right) \mathbf{x}} \\
& \longrightarrow \frac{1}{A} \frac{A^{n+1}}{(2 \pi)^{D(n+1)}} \int_{\mathbb{R}^{D}} \mathrm{~d} \mathbf{x} \int \mathrm{d} \hat{q} \mathcal{A}(\hat{q}) \mathrm{e}^{\mathrm{i}\left(\mathbf{q}^{(0)}+\ldots+\mathbf{q}^{(n)}\right) \mathbf{x}} \\
& =\frac{A^{n}}{(2 \pi)^{D n}} \int \mathrm{d} \hat{q} \delta\left(\mathbf{q}^{(0)}+\ldots+\mathbf{q}^{(n)}\right) \mathcal{A}(\hat{q})=\frac{A^{n}}{(2 \pi)^{D n}} \int \mathrm{d} q_{\perp} \mathcal{A}\left(q_{\perp}\right)
\end{aligned}
$$

Applying this relation to the sum over $\hat{q}_{1}$ in Eq. (C.51), we just have to deal with 
a simple Gaussian integral:

$$
\begin{aligned}
I_{2}=\delta_{\mathbf{q}_{\|\|}, \mathbf{0}} \exp \left(-\hat{q}_{0}^{2} \ell^{2}\left|s_{0}-s_{2}\right|-\frac{\hat{q}_{0}^{2} \tilde{\xi}_{2}^{2}}{2}\right) \\
\quad \times \frac{A^{n}}{(2 \pi)^{D n}} \int \mathrm{d} q_{1 \perp} \exp \left(-q_{1 \perp}^{2}\left(\ell^{2}\left|s_{1}-s_{2}\right|+\frac{\tilde{\xi}_{1}^{2}+\tilde{\xi}_{2}^{2}}{2}\right)\right) \\
\quad \times \exp \left(q_{0 \perp} q_{1 \perp}\left(\ell^{2}\left(\left|s_{0}-s_{1}\right|-\left|s_{0}-s_{2}\right|-\left|s_{1}-s_{2}\right|\right)-\tilde{\xi}_{2}^{2}\right)\right) \\
=\delta_{\mathbf{q}_{0 \|}, \mathbf{0}} \exp \left(-\hat{q}_{0}^{2} \ell^{2}\left|s_{0}-s_{2}\right|-\frac{\hat{q}_{0}^{2} \tilde{\xi}_{2}^{2}}{2}\right) \frac{A^{n}}{(2 \pi)^{D n}}\left(\frac{2 \pi}{\tilde{\xi}_{1}^{2}+\tilde{\xi}_{2}^{2}+2 \ell^{2}\left|s_{1}-s_{2}\right|}\right)^{D n / 2} \\
\quad \times \exp \left(\frac{q_{0 \perp}^{2}\left(\ell^{2}\left(\left|s_{0}-s_{1}\right|-\left|s_{0}-s_{2}\right|-\left|s_{1}-s_{2}\right|\right)-\tilde{\xi}_{2}^{2}\right)^{2}}{2}\right) \\
\left.=\delta_{\mathbf{q}_{0 \|}, \mathbf{0}}\left(\frac{A}{(2 \pi)^{D}}\left(\frac{2 \pi}{\tilde{\xi}_{1}^{2}+\tilde{\xi}_{2}^{2}+2 \ell^{2}\left|s_{1}-s_{2}\right|}\right)^{n}\right)^{n}-s_{1} \mid\right) \\
\quad \times \exp \left(\frac{q_{0 \perp}^{2}}{2}\left\{\frac{\left(\ell^{2} g(\mathbf{s})-\tilde{\xi}_{2}^{2}\right)^{2}}{\tilde{\xi}_{1}^{2}+\tilde{\xi}_{2}^{2}+2 \ell^{2}\left|s_{1}-s_{2}\right|}-2 \ell^{2}\left|s_{0}-s_{2}\right|-\tilde{\xi}_{2}^{2}\right\}\right)
\end{aligned}
$$

where we made the simplifying definition:

$$
g(\mathbf{s}) \equiv g\left(s_{0}, s_{1}, s_{2}\right):=\left|s_{0}-s_{1}\right|-\left|s_{0}-s_{2}\right|-\left|s_{1}-s_{2}\right|
$$

Now we can recompose $I\left(\hat{q}_{0}, s_{0}\right)$, Eq. (C.49), using the expressions (C.50) and (C.54). We also neglect all terms of the form $(\ldots)^{n}=1+\mathcal{O}(n)$, and hence only keep the lowest order in $n$ :

$$
\begin{gathered}
I\left(\hat{q}_{0}, s_{0}\right)=\delta_{\hat{q}_{0}, \hat{0}}+\delta_{\mathbf{q}_{0 \|}, \mathbf{0}} \mu^{2} Q \int_{0}^{1} \mathrm{~d} s_{1} \int_{\xi_{1}^{2}, s_{1}} \exp \left(-\frac{q_{0 \perp}^{2}}{2}\left(2 \ell^{2}\left|s_{0}-s_{1}\right|+\tilde{\xi}_{1}^{2}\right)\right) \\
+\delta_{\mathbf{q}_{0 \|}, \mathbf{0}} \frac{\left(\mu^{2} Q\right)^{2}}{2} \int_{0}^{1} \mathrm{~d} s_{1} \mathrm{~d} s_{2} \int_{\xi_{1}^{2}, s_{1}} \int_{\xi_{2}^{2}, s_{2}} \\
\quad \exp \left(\frac{q_{0 \perp}^{2}}{2}\left\{\frac{\left(\ell^{2} g(\mathbf{s})-\tilde{\xi}_{2}^{2}\right)^{2}}{\tilde{\xi}_{1}^{2}+\tilde{\xi}_{2}^{2}+2 \ell^{2}\left|s_{1}-s_{2}\right|}-2 \ell^{2}\left|s_{0}-s_{2}\right|-\tilde{\xi}_{2}^{2}\right\}\right)+\mathcal{O}\left(Q^{3}\right)
\end{gathered}
$$

and with that:

$$
\begin{aligned}
I(\hat{0}, 0) & =1+\mu^{2} Q \int_{0}^{1} \mathrm{~d} s_{1} \int_{\xi_{1}^{2}, s_{1}} 1+\frac{\left(\mu^{2} Q\right)^{2}}{2} \int_{0}^{1} \mathrm{~d} s_{1} \mathrm{~d} s_{2} \int_{\xi_{1}^{2}, s_{1}} \int_{\xi_{2}^{2}, s_{2}} 1+\mathcal{O}\left(Q^{3}\right) \\
& =1+\mu^{2} Q+\frac{\left(\mu^{2} Q\right)^{2}}{2}+\mathcal{O}\left(Q^{3}\right)
\end{aligned}
$$

Now we can plug these two results for $I\left(\hat{q}_{0}, s_{0}\right)$ and $I(\hat{0}, 0)$ back into the original 
saddle point equation (3.46):

$$
\begin{aligned}
& \left((1-Q) \delta_{\hat{q}_{0}, \hat{0}}+Q \delta_{\mathbf{q}_{0 \|}, \mathbf{0}} \int_{\xi^{2}, s_{0}} \exp \left(-\frac{\hat{q}_{0}^{2} \xi^{2}}{2}\right)\right)\left(1+\mu^{2} Q+\frac{\left(\mu^{2} Q\right)^{2}}{2}\right) \\
& =\delta_{\hat{q}_{0}, \hat{0}}+\delta_{\mathbf{q}_{0 \|}, \mathbf{0}} \mu^{2} Q \int_{0}^{1} \mathrm{~d} s_{1} \int_{\xi_{1}^{2}, s_{1}} \exp \left(-\frac{q_{0 \perp}^{2}}{2}\left(2 \ell^{2}\left|s_{0}-s_{1}\right|+\tilde{\xi}_{1}^{2}\right)\right) \\
& +\delta_{\mathbf{q}_{0 \|}, \mathbf{0}} \frac{\left(\mu^{2} Q\right)^{2}}{2} \int_{0}^{1} \mathrm{~d} s_{1} \mathrm{~d} s_{2} \int_{\xi_{1}^{2}, s_{1}} \int_{\xi_{2}^{2}, s_{2}} \\
& \quad \exp \left(\frac{q_{0 \perp}^{2}}{2}\left\{\frac{\left(\ell^{2} g(\mathbf{s})-\tilde{\xi}_{2}^{2}\right)^{2}}{\tilde{\xi}_{1}^{2}+\tilde{\xi}_{2}^{2}+2 \ell^{2}\left|s_{1}-s_{2}\right|}-2 \ell^{2}\left|s_{0}-s_{2}\right|-\tilde{\xi}_{2}^{2}\right\}\right)+\mathcal{O}\left(Q^{3}\right) .
\end{aligned}
$$

This equation must be valid for any choice of $\hat{q}_{0}$ and $s_{0}$. Let us look at different cases:

For $\hat{q}_{0}=\hat{0}$, we get the trivial relation

$$
\begin{aligned}
(1-Q)+Q & =1 \\
1 & =1
\end{aligned}
$$

as one can already see at the saddle point equation (3.46). For $\hat{q}_{0} \neq \hat{0}$ and $\mathbf{q}_{0 \|} \neq \mathbf{0}$, all terms in Eq. (C.58) vanish due to the $\delta$-constraints and we also get the trivial result $0=0$.

Hence, from here, we restrict ourselves to the more interesting case $\hat{q}_{0} \neq \hat{0}$ and $\mathbf{q}_{0 \|}=\mathbf{0}$. Furthermore, to be consistent with the terms on the left hand side, we also insert the substitution $\tilde{\xi}^{2}=\xi^{2}+a^{2}$ (see Eq. (3.49)) on the left hand side of Eq. (C.58), again:

$$
\begin{aligned}
& Q \int_{\xi^{2}, s_{0}} \exp \left(-\frac{q_{0 \perp}^{2} \xi^{2}}{2}\right)\left(1+\mu^{2} Q+\frac{\left(\mu^{2} Q\right)^{2}}{2}\right) \\
& =\mu^{2} Q \int_{0}^{1} \mathrm{~d} s_{1} \int_{\xi_{1}^{2}, s_{1}} \exp \left(-\frac{q_{0 \perp}^{2}}{2}\left(2 \ell^{2}\left|s_{0}-s_{1}\right|+a^{2}+\xi_{1}^{2}\right)\right) \\
& +\frac{\left(\mu^{2} Q\right)^{2}}{2} \int_{0}^{1} \mathrm{~d} s_{1} \mathrm{~d} s_{2} \int_{\xi_{1}^{2}, s_{1}} \int_{\xi_{2}^{2}, s_{2}} \\
& \quad \exp \left(\frac{q_{0 \perp}^{2}}{2}\left\{\frac{\left(\ell^{2} g(\mathbf{s})-a^{2}-\xi_{2}^{2}\right)^{2}}{\xi_{1}^{2}+\xi_{2}^{2}+2 a^{2}+2 \ell^{2}\left|s_{1}-s_{2}\right|}-2 \ell^{2}\left|s_{0}-s_{2}\right|-a^{2}-\xi_{2}^{2}\right\}\right) \\
& +\mathcal{O}\left(Q^{3}\right) .
\end{aligned}
$$

The canceled term in the first line is of the order $Q^{3}$ and thus can be neglected. 


\section{C.5 Obtaining the Equation for the Localization Length}

\section{C.5.1 Normalization of Length Scales}

As anticipated in Sec. 3.7.1, we can simplify the saddle point equation by measuring all lengths in units of $\ell$, i.e. define:

$$
\begin{aligned}
\xi_{\ell}^{2} & :=\xi^{2} / \ell^{2} \\
a_{\ell}^{2} & :=a^{2} / \ell^{2} \\
q_{0 \ell \perp}^{2} & :=\ell^{2} q_{0 \perp}^{2}
\end{aligned}
$$

With the new variables, Eq. (3.51) does not exhibit an explicit dependence on $\ell$ :

$$
\begin{aligned}
(1+ & \left.\mu^{2} Q\right) \int_{\xi^{2}, s_{0}} \exp \left(-\frac{q_{0 \ell \perp}^{2} \xi_{\ell}^{2}}{2}\right) \\
= & \mu^{2} \int_{0}^{1} \mathrm{~d} s_{1} \int_{\xi_{1}^{2}, s_{1}} \exp \left(-\frac{q_{0 \ell \perp}^{2}}{2}\left(2\left|s_{0}-s_{1}\right|+a_{\ell}^{2}+\xi_{1 \ell}^{2}\right)\right) \\
& +\frac{\mu^{4} Q}{2} \int_{0}^{1} \mathrm{~d} s_{1} \mathrm{~d} s_{2} \int_{\xi_{1}^{2}, s_{1}} \int_{\xi_{2}^{2}, s_{2}} \\
& \quad \exp \left(\frac{q_{0 \ell \perp}^{2}}{2}\left\{\frac{\left(g(\mathbf{s})-a_{\ell}^{2}-\xi_{2 \ell}^{2}\right)^{2}}{\xi_{1 \ell}^{2}+\xi_{2 \ell}^{2}+2 a_{\ell}^{2}+2\left|s_{1}-s_{2}\right|}-2\left|s_{0}-s_{2}\right|-a_{\ell}^{2}-\xi_{2 \ell}^{2}\right\}\right) \\
& +\mathcal{O}\left(Q^{2}\right) .
\end{aligned}
$$

Now we normalize the localization length with $\varepsilon$ and therefore, introduce the new variable

$$
\begin{aligned}
& \theta:=\frac{f}{\varepsilon \xi_{\ell}^{2}}=\frac{f \ell^{2}}{\left(\mu^{2}-1\right) \xi^{2}} \quad \Longleftrightarrow \quad \xi_{\ell}^{2}=\frac{f}{\varepsilon \theta}, \\
& \text { with } f=\frac{2}{3}+a_{\ell}^{2}=\frac{2}{3}+\frac{a^{2}}{\ell^{2}}
\end{aligned}
$$

which - as we will see - remains of the order $\mathcal{O}(1)$, when we approach the transition point. Since the localization lengths are very large, we also normalize $q_{0 \ell \perp}^{2}$ with $\varepsilon$, so that we have to deal with variables of the order $\mathcal{O}(1)$. Hence we define:

$$
t:=\frac{f q_{0 \ell \perp}^{2}}{2 \varepsilon} \quad \Longleftrightarrow \quad \frac{q_{0 \ell \perp}^{2}}{2}=\frac{t \varepsilon}{f}
$$


With that Eq. (C.62) becomes:

$$
\begin{aligned}
(1+ & \left.\mu^{2} Q\right) \int_{\theta, s_{0}} \exp \left(-\frac{t}{\theta}\right) \\
= & \mu^{2} \int_{0}^{1} \mathrm{~d} s_{1} \int_{\theta_{1}, s_{1}} \exp \left(-t\left\{\frac{1}{\theta_{1}}+\frac{2 \varepsilon}{f}\left|s_{0}-s_{1}\right|+\frac{\varepsilon}{f} a_{\ell}^{2}\right\}\right) \\
& +\frac{\mu^{4} Q}{2} \int_{0}^{1} \mathrm{~d} s_{1} \mathrm{~d} s_{2} \int_{\theta_{1}, s_{1}} \int_{\theta_{2}, s_{2}} \\
& \exp \left(\frac{t}{f}\left\{\frac{\left(\varepsilon g(\mathbf{s})-\varepsilon a_{\ell}^{2}-f \theta_{2}^{-1}\right)^{2}}{f \theta_{1}^{-1}+f \theta_{2}^{-1}+2 \varepsilon a_{\ell}^{2}+2 \varepsilon\left|s_{1}-s_{2}\right|}-2 \varepsilon\left|s_{0}-s_{2}\right|-\varepsilon a_{\ell}^{2}-f \theta_{2}^{-1}\right\}\right) \\
+ & \mathcal{O}\left(Q^{2}\right) .
\end{aligned}
$$

Here we also turned the probability density $\mathcal{P}\left(\xi^{2}, s\right)$ for $\xi^{2}$ into the probability density $\pi(\theta, s)$ for $\theta$ :

$$
\begin{aligned}
& \int_{\xi^{2}, s}=\int_{0}^{\infty} \mathrm{d} \xi^{2} \mathcal{P}\left(\xi^{2}, s\right) \longrightarrow \int_{\theta, s}=\int_{0}^{\infty} \mathrm{d} \theta \pi(\theta, s) . \\
& \text { with } \mathrm{d} \xi^{2} \mathcal{P}\left(\xi^{2}, s\right)=\mathrm{d} \theta \pi(\theta, s)
\end{aligned}
$$

Now, we can sort the saddle point equation (C.65) in orders of $\varepsilon$, and have to keep in mind that $Q=\mathcal{O}(\varepsilon)$, as found in Eq. (3.58).

For the three exponentials, we can expand all expressions which are of the order $\mathcal{O}(\varepsilon)$ : In doing so, we note that the last term on the right hand side of Eq. (C.65) has a prefactor $Q$ and thus already is of the order $\mathcal{O}(\varepsilon)$. Hence, we only need to expand the exponential up to zeroth order, i.e. neglect all terms of the order $\mathcal{O}(\varepsilon)$ :

$$
\begin{aligned}
& \left(1+\mu^{2} Q\right) \int_{\theta, s_{0}} \exp \left(-\frac{t}{\theta}\right) \\
& =\mu^{2} \int_{0}^{1} \mathrm{~d} s_{1} \int_{\theta_{1}, s_{1}} \exp \left(-\frac{t}{\theta_{1}}\right)\left(1-t\left\{\frac{2 \varepsilon}{f}\left|s_{0}-s_{1}\right|+\frac{\varepsilon}{f} a_{\ell}^{2}\right\}\right) \\
& \quad+\frac{\mu^{4} Q}{2} \int_{0}^{1} \mathrm{~d} s_{1} \mathrm{~d} s_{2} \int_{\theta_{1}, s_{1}} \int_{\theta_{2}, s_{2}} \exp \left(\frac{t}{f}\left\{\frac{\left(f \theta_{2}^{-1}\right)^{2}}{f \theta_{1}^{-1}+f \theta_{2}^{-1}}-f \theta_{2}^{-1}\right\}\right)+\mathcal{O}\left(\varepsilon^{2}\right) .
\end{aligned}
$$

Here, the argument of the last exponential can be simplified:

$$
\begin{aligned}
& \frac{t}{f}\left\{\frac{\left(f \theta_{2}^{-1}\right)^{2}}{f \theta_{1}^{-1}+f \theta_{2}^{-1}}-f \theta_{2}^{-1}\right\}=t\left\{\frac{\left(\theta_{2}^{-1}\right)^{2}}{\theta_{1}^{-1}+\theta_{2}^{-1}}-\theta_{2}^{-1}\right\}=t \frac{\left(\theta_{2}^{-1}\right)^{2}-\theta_{2}^{-1} \theta_{1}^{-1}-\left(\theta_{2}^{-1}\right)^{2}}{\theta_{1}^{-1}+\theta_{2}^{-1}} \\
& =-t \frac{1}{\theta_{1}+\theta_{2}} .
\end{aligned}
$$


With that the saddle point equation (C.67) becomes:

$$
\begin{aligned}
& \left(1+\mu^{2} Q\right) \int_{\theta_{1}, s_{0}} \exp \left(-\frac{t}{\theta_{1}}\right) \\
& =\mu^{2} \int_{0}^{1} \mathrm{~d} s_{1} \int_{\theta_{1}, s_{1}} \exp \left(-\frac{t}{\theta_{1}}\right)\left(1-2 t \frac{\varepsilon}{f}\left\{\left|s_{0}-s_{1}\right|+\frac{1}{2} a_{\ell}^{2}\right\}\right) \\
& \quad+\frac{\mu^{4} Q}{2} \int_{0}^{1} \mathrm{~d} s_{1} \mathrm{~d} s_{2} \int_{\theta_{1}, s_{1}} \int_{\theta_{2}, s_{2}} \exp \left(-\frac{t}{\theta_{1}+\theta_{2}}\right)+\mathcal{O}\left(\varepsilon^{2}\right) .
\end{aligned}
$$

\section{C.5.2 Laplace-Transformation of the Saddle Point Equation}

Eq. (C.69) could in principle be used to determine the distribution $\pi(\theta)$. When calculating the actual shape of this distribution, however, is much more convenient, if it has the form of a differential (or integro-differential) equation, and does not involve an external variable $t$. Therefore, as a next step, we try to convert the three exponential functions to delta functions with the respective $\theta$ as argument. This is done by a Laplace transformation: We multiply the whole equation with $\sin (a t) \exp \left(t \theta^{-1}\right)$, integrate over $t$ and take the limit $a \rightarrow 0$. Then we can use the following relations, which are valid for any $\Theta>0$ :

$$
\begin{aligned}
& \lim _{a \rightarrow 0} \int_{0}^{\infty} \mathrm{d} t \sin (a t) \exp \left(\frac{t}{\theta}\right) \exp \left(-\frac{t}{\Theta}\right)=\lim _{a \rightarrow 0} \frac{a}{a^{2}+\left(\Theta^{-1}-\theta^{-1}\right)^{2}} \\
& =\delta\left(\Theta^{-1}-\theta^{-1}\right)
\end{aligned}
$$

and

$$
\begin{aligned}
& -\lim _{a \rightarrow 0} \int_{0}^{\infty} \mathrm{d} t t \cdot \sin (a t) \exp \left(\frac{t}{\theta}\right) \exp \left(-\frac{t}{\Theta}\right) \\
& =\lim _{a \rightarrow 0} \frac{\partial}{\partial \Theta^{-1}} \int_{0}^{\infty} \mathrm{d} t \sin (a t) \exp \left(\frac{t}{\theta}\right) \exp \left(-\frac{t}{\Theta}\right) \\
& =\lim _{a \rightarrow 0} \frac{\partial}{\partial \Theta^{-1}} \frac{a}{a^{2}+\left(\Theta^{-1}-\theta^{-1}\right)^{2}} \\
& =\delta^{\prime}\left(\Theta^{-1}-\theta^{-1}\right)
\end{aligned}
$$

Now, we apply those two relations to Eq. (C.69). In doing so, $\Theta=\theta_{1}$ in the first two terms, and $\Theta=\theta_{1}+\theta_{2}$ in the last term:

$$
\begin{aligned}
(1+ & \left.\mu^{2} Q\right) \int_{0}^{\infty} \mathrm{d} \theta_{1} \pi\left(\theta_{1}, s_{0}\right) \delta\left(\theta_{1}^{-1}-\theta^{-1}\right) \\
= & \mu^{2} \int_{0}^{1} \mathrm{~d} s_{1} \int_{0}^{\infty} \mathrm{d} \theta_{1} \pi\left(\theta_{1}, s_{1}\right)\left(\delta\left(\theta_{1}^{-1}-\theta^{-1}\right)+\frac{2 \varepsilon}{f} \delta^{\prime}\left(\theta_{1}^{-1}-\theta^{-1}\right)\left\{\left|s_{0}-s_{1}\right|+\frac{1}{2} a_{\ell}^{2}\right\}\right) \\
& +\frac{\mu^{4} Q}{2} \int_{0}^{1} \mathrm{~d} s_{1} \mathrm{~d} s_{2} \int_{0}^{\infty} \mathrm{d} \theta_{1} \pi\left(\theta_{1}, s_{1}\right) \int_{0}^{\infty} \mathrm{d} \theta_{2} \pi\left(\theta_{2}, s_{2}\right) \delta\left(\left(\theta_{1}+\theta_{2}\right)^{-1}-\theta^{-1}\right) \\
& +\mathcal{O}\left(\varepsilon^{2}\right) .
\end{aligned}
$$


Now we want to integrate over $\theta_{1}$ in the first two terms and over $\theta_{2}$ in the last term (marked in blue). Therefore we need to use the following relations for $\delta$-functions:

$$
\begin{aligned}
\int \mathrm{d} \theta_{1} \pi\left(\theta_{1}\right) \delta\left(g\left(\theta_{1}\right)\right) & =\sum_{\nu} \frac{\pi\left(\theta_{1 \nu}\right)}{\left|g^{\prime}\left(\theta_{1 \nu}\right)\right|} \\
\int \mathrm{d} \theta_{1} \pi\left(\theta_{1}\right) \delta^{\prime}\left(g\left(\theta_{1}\right)\right) & =\sum_{\nu} \frac{\pi\left(\theta_{1 \nu}\right) g^{\prime \prime}\left(\theta_{1 \nu}\right)-\pi^{\prime}\left(\theta_{1 \nu}\right) g^{\prime}\left(\theta_{1 \nu}\right)}{\left|g^{\prime}\left(\theta_{1 \nu}\right)\right|^{3}},
\end{aligned}
$$

where $\theta_{1 \nu}$ are the roots and $g^{\prime}\left(\theta_{1 \nu}\right)$ and $g^{\prime \prime}\left(\theta_{1 \nu}\right)$ the derivatives of the function $g\left(\theta_{1}\right)$, which we now find for the presented case:

\section{- In the first two terms:}

$$
\begin{aligned}
& g\left(\theta_{1}\right)=\theta_{1}^{-1}-\theta^{-1} \stackrel{!}{=} 0 \\
& \Rightarrow \text { root: } \theta_{1,0}=\theta \\
& \text { derivatives: } g^{\prime}\left(\theta_{1}\right)=-\theta_{1}^{-2} \\
& \qquad g^{\prime \prime}\left(\theta_{1}\right)=2 \theta_{1}^{-3}
\end{aligned}
$$

\section{- In the last term:}

$$
\begin{aligned}
& g\left(\theta_{2}\right)=\left(\theta_{1}+\theta_{2}\right)^{-1}-\theta^{-1} \stackrel{!}{=} 0 \\
& \Leftrightarrow \theta \stackrel{!}{=} \theta_{1}+\theta_{2} \\
& \Rightarrow \text { root: } \begin{cases}\theta_{2,0}=\theta-\theta_{1} & \text { for } \theta \geq \theta_{1} \\
\text { no root } & \text { for } \theta<\theta_{1}\end{cases}
\end{aligned}
$$

derivative: $g^{\prime}\left(\theta_{2}\right)=-\left(\theta_{1}+\theta_{2}\right)^{-2}, g^{\prime}\left(\theta_{2,0}\right)=-\theta^{-2}$

We plug Eqs. (C.72) with the found root and derivatives (C.73) back into the saddle point equation (C.71):

$$
\begin{aligned}
& \left(1+\mu^{2} Q\right) \frac{\pi\left(\theta, s_{0}\right)}{\left|-\theta^{-2}\right|} \\
& =\mu^{2} \int_{0}^{1} \mathrm{~d} s_{1}\left(\frac{\pi\left(\theta, s_{1}\right)}{\left|-\theta^{-2}\right|}+\frac{2 \varepsilon}{f} \frac{\pi\left(\theta, s_{1}\right) \cdot 2 \theta^{-3}+\partial_{\theta} \pi\left(\theta, s_{1}\right) \cdot \theta^{-2}}{\left|-\theta^{-2}\right|^{3}}\left\{\left|s_{0}-s_{1}\right|+\frac{1}{2} a_{\ell}^{2}\right\}\right) \\
& \quad+\frac{\mu^{4} Q}{2} \int_{0}^{1} \mathrm{~d} s_{1} \mathrm{~d} s_{2} \int_{0}^{\infty} \mathrm{d} \theta_{1} \pi\left(\theta_{1}, s_{1}\right) \frac{\pi\left(\theta-\theta_{1}, s_{2}\right)}{\left|-\theta^{-2}\right|}+\mathcal{O}\left(\varepsilon^{2}\right),
\end{aligned}
$$

where $\partial_{\theta} \pi\left(\theta, s_{1}\right)$ is the derivative of $\pi\left(\theta, s_{1}\right)$ with respect to $\theta$. We now multiply this equation with $\theta^{-2}$ on both sides, plug in $\mu^{2}=1+\varepsilon$ and $Q=2 \varepsilon+\mathcal{O}\left(\varepsilon^{2}\right)$, Eq. (3.58), and get:

$$
\begin{aligned}
& (1+2 \varepsilon) \pi\left(\theta, s_{0}\right) \\
& =(1+\varepsilon) \int_{0}^{1} \mathrm{~d} s_{1}\left(\pi\left(\theta, s_{1}\right)+\frac{2 \varepsilon}{f}\left(2 \theta \pi\left(\theta, s_{1}\right)+\theta^{2} \partial_{\theta} \pi\left(\theta, s_{1}\right)\right)\left\{\left|s_{0}-s_{1}\right|+\frac{1}{2} a_{\ell}^{2}\right\}\right) \\
& \quad+\varepsilon \int_{0}^{1} \mathrm{~d} s_{1} \mathrm{~d} s_{2} \int_{0}^{\infty} \mathrm{d} \theta_{1} \pi\left(\theta_{1}, s_{1}\right) \pi\left(\theta-\theta_{1}, s_{2}\right)+\mathcal{O}\left(\varepsilon^{2}\right),
\end{aligned}
$$


and by substituting $s_{0} \rightarrow s$ :

$$
\begin{aligned}
& (1+2 \varepsilon) \pi(\theta, s) \\
& =(1+\varepsilon) \int_{0}^{1} \mathrm{~d} s_{1} \pi\left(\theta, s_{1}\right)+\frac{\varepsilon}{f} \int_{0}^{1} \mathrm{~d} s_{1} \partial_{\theta}\left(\theta^{2} \pi\left(\theta, s_{1}\right)\right)\left\{2\left|s-s_{1}\right|+a_{\ell}^{2}\right\} \\
& \quad+\varepsilon \int_{0}^{1} \mathrm{~d} s_{1} \mathrm{~d} s_{2} \int_{0}^{\theta} \mathrm{d} \theta_{1} \pi\left(\theta_{1}, s_{1}\right) \pi\left(\theta-\theta_{1}, s_{2}\right)+\mathcal{O}\left(\varepsilon^{2}\right)
\end{aligned}
$$

In Sec. 3.7 we examine this integro-differential equation and display the actual shape of $\pi(\theta, s)$.

\section{C.6 Calculation of a Correlator}

Throughout chapter 3 about cross-linked directed polymers we need expressions of the form:

$$
\begin{gathered}
\left\langle\exp \left(\mathrm{i} \sum_{j=1}^{J} \hat{q}_{j} \hat{r}\left(z_{j}\right)\right)\right\rangle_{n+1}^{H_{\text {align }}+H_{\text {tilt }}}=\int \mathcal{D} \hat{r}(z) \mathrm{e}^{-H_{\text {align }, 1}^{(n+1)}-H_{\text {tilt }, 1}^{(n+1)}} \exp \left(\mathrm{i} \sum_{j=1}^{J} \hat{q}_{j} \hat{r}\left(z_{j}\right)\right)= \\
\int \mathrm{d} \hat{r}_{1} \cdots \mathrm{d} \hat{r}_{Z_{L}} \exp \left(\mathrm{i} \sum_{j=1}^{J} \hat{q}_{j} \hat{r}_{Z_{j}}-\frac{\sigma \Delta z}{2} \sum_{Z=2}^{Z_{L}} \frac{\left(\hat{r}_{Z}-\hat{r}_{Z-1}\right)^{2}}{\Delta z^{2}}-\Delta z \sum_{Z=2}^{Z_{L}} \frac{\hat{h}_{Z}\left(\hat{r}_{Z}-\hat{r}_{Z-1}\right)}{\Delta z}\right) \\
\int \mathrm{d} \hat{r}_{1} \cdots \mathrm{d} \hat{r}_{Z_{L}} \exp \left(-\frac{\sigma \Delta z}{2} \sum_{Z=2}^{Z_{L}} \frac{\left(\hat{r}_{Z}-\hat{r}_{Z-1}\right)^{2}}{\Delta z^{2}}\right)
\end{gathered}
$$

First, let us look at the denominator: we can keep $\hat{r}_{1}$, but make the substitution $\hat{t}_{Z}=\hat{r}_{Z}-\hat{r}_{Z-1}$ for $Z=2, \ldots, Z_{L}$, and write:

$$
\begin{aligned}
\mathcal{D} & =\int \mathrm{d} \hat{r}_{1} \cdots \mathrm{d} \hat{r}_{Z_{L}} \exp \left(-\frac{\sigma \Delta z}{2} \sum_{Z=2}^{Z_{L}} \frac{\left(\hat{r}_{Z}-\hat{r}_{Z-1}\right)^{2}}{\Delta z^{2}}\right) \\
& =\int \mathrm{d} \hat{r}_{1} \mathrm{~d} \hat{t}_{2} \cdots \mathrm{d} \hat{t}_{Z_{L}} \exp \left(-\frac{\sigma \Delta z}{2} \sum_{Z=2}^{Z_{L}} \frac{\hat{t}_{Z}^{2}}{\Delta z^{2}}\right) \\
& =A^{n+1}\left(\int \mathrm{d} \hat{t} \exp \left(-\frac{\sigma}{2 \Delta z} \hat{t}^{2}\right)\right)^{Z_{L}-1} \\
& =A^{n+1}\left(\frac{2 \pi \Delta z}{\sigma}\right)^{D(n+1)\left(Z_{L}-1\right)}
\end{aligned}
$$


In the numerator we make the same substitution and get:

$$
\begin{aligned}
& \mathcal{N}= \\
& \int \mathrm{d} \hat{r}_{1} \cdots \mathrm{d} \hat{r}_{Z_{L}} \exp \left(\mathrm{i} \sum_{j=1}^{J} \hat{q}_{j} \hat{r}_{Z_{j}}-\frac{\sigma \Delta z}{2} \sum_{Z=2}^{Z_{L}} \frac{\left(\hat{r}_{Z}-\hat{r}_{Z-1}\right)^{2}}{\Delta z^{2}}-\Delta z \sum_{Z=2}^{Z_{L}} \frac{\hat{h}_{Z}\left(\hat{r}_{Z}-\hat{r}_{Z-1}\right)}{\Delta z}\right) \\
& =\int \mathrm{d} \hat{r}_{1} \mathrm{~d} \hat{t}_{2} \cdots \mathrm{d} \hat{t}_{Z_{L}} \exp \left(\mathrm{i} \sum_{j=1}^{J} \hat{q}_{j}\left(\hat{r}_{1}+\hat{t}_{2}+\ldots+\hat{t}_{Z_{j}}\right)-\frac{\sigma}{2 \Delta z} \sum_{Z=2}^{Z_{L}} \hat{t}_{Z}^{2}-\sum_{Z=2}^{Z_{L}} \hat{h}_{Z} \hat{t}_{Z}\right) \\
& =\int \mathrm{d} \hat{r}_{1} \exp \left(\mathrm{i} \hat{r}_{1} \sum_{j=1}^{J} \hat{q}_{j}\right) \\
& \quad \times \int \mathrm{d} \hat{t}_{2} \cdots \mathrm{d} \hat{t}_{Z_{L}} \exp \left(\mathrm{i} \sum_{j=1}^{J} \hat{q}_{j}\left(\hat{t}_{2}+\ldots+\hat{t}_{Z_{j}}\right)-\frac{\sigma}{2 \Delta z} \sum_{Z=2}^{Z_{L}} \hat{t}_{Z}^{2}-\sum_{Z=2}^{Z_{L}} \hat{h}_{Z} \hat{t}_{Z}\right) \quad \text { (C.79) }
\end{aligned}
$$

In the last two lines, the integral over $\hat{r}_{1}$ can easily performed, yielding $A^{n+1} \delta_{\hat{q}_{1}+\ldots+\hat{q}_{J}, \hat{0}}$. For the integral over $\hat{t}_{2}, \ldots, \hat{t}_{Z_{L}}$, we make the definition:

$$
\begin{array}{r}
\hat{h}_{Z}^{\prime}:=\hat{h}_{Z}-\mathrm{i} \sum_{j=1}^{J} \hat{q}_{j} \theta_{Z_{j}, Z} \\
\text { with } \theta_{Z_{j}, Z}=\left\{\begin{array}{lll}
1 & \text { if } & Z_{j} \geq Z \\
0 & \text { if } & Z_{j}<Z
\end{array}\right.
\end{array}
$$

With that the numerator $\mathcal{N}$ can be simplified:

$$
\begin{aligned}
\mathcal{N} & =A^{n+1} \delta_{\hat{q}_{1}+\ldots+\hat{q}_{J}, \hat{0}} \int \mathrm{d} \hat{t}_{2} \cdots \mathrm{d} \hat{t}_{Z_{L}} \exp \left(-\frac{\sigma}{2 \Delta z} \sum_{Z=2}^{Z_{L}} \hat{t}_{Z}^{2}-\sum_{Z=2}^{Z_{L}} \hat{h}_{Z}^{\prime} \hat{t}_{Z}\right) \\
& =A^{n+1} \delta_{\hat{q}_{1}+\ldots+\hat{q}_{J}, \hat{0}} \prod_{Z=2}^{Z_{L}}\left\{\left(\frac{2 \pi \Delta z}{\sigma}\right)^{D(n+1)} \exp \left(\frac{\hat{h}_{Z}^{\prime 2}}{4 \sigma /(2 \Delta z)}\right)\right\} \\
& =A^{n+1} \delta_{\hat{q}_{1}+\ldots+\hat{q}_{J}, \hat{0}}\left(\frac{2 \pi \Delta z}{\sigma}\right)^{D(n+1)\left(Z_{L}-1\right)} \exp \left(\frac{\Delta z}{2 \sigma} \sum_{Z=2}^{Z_{L}} \hat{h}_{Z}^{\prime 2}\right)
\end{aligned}
$$


With that expression for the numerator and (C.78) for the denominator, we get for the original expression (C.77)

$$
\begin{aligned}
\left\langle\exp \left(\mathrm{i} \sum_{j=1}^{J} \hat{q}_{j} \hat{r}\left(z_{j}\right)\right)\right\rangle_{n+1}^{H_{\text {align }}+H_{\text {tilt }}} & A^{n+1} \delta_{\hat{q}_{1}+\ldots+\hat{q}_{J}, \hat{0}}\left(\frac{2 \pi \Delta z}{\sigma}\right)^{D(n+1)\left(Z_{L}-1\right)} \exp \left(\frac{\Delta z}{2 \sigma} \sum_{Z=2}^{Z_{L}} \hat{h}_{Z}^{\prime 2}\right) \\
= & A^{n+1}\left(\frac{2 \pi \Delta z}{\sigma}\right)^{D(n+1)\left(Z_{L}-1\right)} \\
= & \delta_{\hat{q}_{1}+\ldots+\hat{q}_{J}, \hat{0}} \exp \left(\frac{\Delta z}{2 \sigma} \sum_{Z=2}^{Z_{L}} \hat{h}_{Z}^{\prime 2}\right) \\
= & \delta_{\hat{q}_{1}+\ldots+\hat{q}_{J}, \hat{0}} \exp \left(\frac{\Delta z}{2 \sigma} \sum_{Z=2}^{Z_{L}}\left(\hat{h}_{Z}-\mathrm{i} \sum_{j=1}^{J} \hat{q}_{j} \theta_{Z_{j}, Z}\right)^{2}\right)
\end{aligned}
$$

Changing back to the Riemann-integral by taking $\Delta z=L / Z_{L} \rightarrow 0$ we get:

$$
\begin{aligned}
& \left\langle\exp \left(\mathrm{i} \sum_{j=1}^{J} \hat{q}_{j} \hat{r}\left(z_{j}\right)\right)\right\rangle_{n+1}^{H_{\text {align }}+H_{\mathrm{tilt}}} \\
& \quad=\delta_{\hat{q}_{1}+\ldots+\hat{q}_{J}, \hat{0}} \exp \left(\frac{1}{2 \sigma} \int_{0}^{L} \mathrm{~d} z\left(\hat{h}(z)-\mathrm{i} \sum_{j=1}^{J} \hat{q}_{j} \theta\left(z_{j}-z\right)\right)^{2}\right)
\end{aligned}
$$

The integral in the exponential can be simplified further. Therefore we will need the following expression (under the restriction $\hat{q}_{1}+\ldots+\hat{q}_{J}=\hat{0}$ ):

$$
\begin{aligned}
& \int_{0}^{L} \mathrm{~d} z\left(\sum_{j=1}^{J} \hat{q}_{j} \theta\left(z_{j}-z\right)\right)^{2} \\
= & \int_{0}^{L} \mathrm{~d} z\left(\sum_{j=1}^{J} \hat{q}_{j}^{2} \theta\left(z_{j}-z\right)+\sum_{j \neq k} \hat{q}_{j} \hat{q}_{k} \theta\left(z_{j}-z\right) \theta\left(z_{k}-z\right)\right) \\
= & \int_{0}^{L} \mathrm{~d} z\left(-\sum_{j \neq k} \hat{q}_{j} \hat{q}_{k} \theta\left(z_{j}-z\right)+\sum_{j \neq k} \hat{q}_{j} \hat{q}_{k} \theta\left(z_{j}-z\right) \theta\left(z_{k}-z\right)\right) \\
= & \int_{0}^{L} \mathrm{~d} z\left(-\sum_{j \neq k} \hat{q}_{j} \hat{q}_{k} \theta\left(z_{j}-z\right)\left(1-\theta\left(z_{k}-z\right)\right)\right) \\
= & -\sum_{j<k} \hat{q}_{j} \hat{q}_{k}\left|z_{j}-z_{k}\right|
\end{aligned}
$$


Despite the minus-sign, this expression is always $\geq 0$, as it can be seen in the first line. With that expression we can calculate the integral in expression (C.84):

$$
\begin{aligned}
& \int_{0}^{L} \mathrm{~d} z\left(\hat{h}(z)-\mathrm{i} \sum_{j=1}^{J} \hat{q}_{j} \theta\left(z_{j}-z\right)\right)^{2} \\
= & \int_{0}^{L} \mathrm{~d} z \hat{h}^{2}(z)-2 \mathrm{i} \sum_{j=1}^{J} \hat{q}_{j} \int_{0}^{L} \mathrm{~d} z \hat{h}(z) \theta\left(z_{j}-z\right)-\int_{0}^{L} \mathrm{~d} z\left(\sum_{j=1}^{J} \hat{q}_{j} \theta\left(z_{j}-z\right)\right)^{2} \\
= & L H^{2}-2 \mathrm{i} \sum_{j=1}^{J} \hat{q}_{j} \hat{H}\left(z_{j}\right)+\sum_{j<k} \hat{q}_{j} \hat{q}_{k}\left|z_{j}-z_{k}\right|
\end{aligned}
$$

where

$$
\hat{H}(z):=\int_{0}^{z} \mathrm{~d} z^{\prime} \hat{h}\left(z^{\prime}\right) \quad \text { and } \quad H^{2}:=\frac{1}{L} \int_{0}^{L} \mathrm{~d} z \hat{h}^{2}(z)
$$

Now, we plug that form back into Eq. (C.84) and get the result:

$$
\begin{aligned}
& \left\langle\exp \left(\mathrm{i} \sum_{j=1}^{J} \hat{q}_{j} \hat{r}\left(z_{j}\right)\right)\right\rangle_{n+1}^{H_{\text {align }}+H_{\text {tilt }}} \\
& \quad=\delta_{\hat{q}_{1}+\ldots+\hat{q}_{J}, \hat{0}} \exp \left(\frac{1}{2 \sigma}\left(L H^{2}-2 \mathrm{i} \sum_{j=1}^{J} \hat{q}_{j} \hat{H}\left(z_{j}\right)+\sum_{j<k} \hat{q}_{j} \hat{q}_{k}\left|z_{j}-z_{k}\right|\right)\right)
\end{aligned}
$$

without the tilting interaction, i.e. $\hat{h}(z) \equiv \hat{0}$, the result simplifies to:

$$
\left\langle\exp \left(\mathrm{i} \sum_{j=1}^{J} \hat{q}_{j} \hat{r}\left(z_{j}\right)\right)\right\rangle_{n+1}^{H_{\text {align }}}=\delta_{\hat{q}_{1}+\ldots+\hat{q}_{J}, \hat{0}} \exp \left(\frac{1}{2 \sigma} \sum_{j<k} \hat{q}_{j} \hat{q}_{k}\left|z_{j}-z_{k}\right|\right)
$$

\section{C.7 The Average Cross-Link Density}

Now we calculate the disorder-averaged number of cross-links $[M]$ and its variance for the directed polymer model. We calculate expectation values of the form $[M(M-1) \cdots(M-J+1)]=: \mathcal{M}_{J}$, in analogy to the RLP model, Sec. 2.9.1. The difference is that the disorder average, defined in Eq. (3.22), additionally involves the integrals over the cross-link heights $z_{e}$. For simplification we define 


$$
\begin{aligned}
& \mathcal{D} \Gamma=\mathcal{D} \mathbf{r}_{1}(z) \cdots \mathcal{D} \mathbf{r}_{N}(z) \text { and get: } \\
& \mathcal{M}_{J}=\frac{1}{\mathcal{Z}_{1}} \sum_{M=0}^{\infty} \sum_{\substack{i_{1}, \ldots, i_{M}=1 \\
j_{1}, \ldots, j_{M}=1}}^{N} \frac{\left(\tilde{\mu}^{2}\right)^{M}}{M ! L^{M}} \int_{0}^{L} \mathrm{~d} z_{1} \cdots \mathrm{d} z_{M} Z(\mathcal{C}) \cdot M(M-1) \cdots(M-J+1) \\
& =\frac{1}{\mathcal{Z}_{1}} \sum_{M=J}^{\infty} \sum_{\substack{i_{1}, \ldots, i_{M}=1 \\
j_{1}, \ldots, j_{M}=1}}^{N} \frac{\left(\tilde{\mu}^{2} / L\right)^{M}}{M !} \frac{M !}{(M-J) !} \int_{0}^{L} \mathrm{~d} z_{1} \cdots \mathrm{d} z_{M} Z\left(\left\{i_{e}, j_{e}, z_{e}\right\}_{e=1}^{M}\right) \\
& =\frac{1}{\mathcal{Z}_{1}} \sum_{M=0}^{\infty} \sum_{\substack{i_{1}, \ldots, i_{M+J}=1 \\
j_{1}, \ldots, j_{M+J}=1}}^{N} \frac{\left(\tilde{\mu}^{2} / L\right)^{M+J}}{(M+J-J) !} \int_{0}^{L} \mathrm{~d} z_{1} \cdots \mathrm{d} z_{M+J} Z\left(\left\{i_{e}, j_{e}, z_{e}\right\}_{e=1}^{M+J}\right) \\
& =\frac{1}{\mathcal{Z}_{1}} \int \mathcal{D} \Gamma \mathrm{e}^{-H_{\mathrm{ev}}-H_{\text {align }}} \sum_{M=0}^{\infty} \sum_{\substack{i_{1}, \ldots, i_{M+J}=1 \\
j_{1}, \ldots, j_{M+J}=1}}^{N} \frac{\left(\tilde{\mu}^{2} / L\right)^{M+J}}{M !} \\
& \int_{0}^{L} \mathrm{~d} z_{1} \cdots \mathrm{d} z_{M+J} \exp \left(-\frac{1}{2 a^{2}} \sum_{e=1}^{M+J}\left(\mathbf{r}_{i_{e}}\left(z_{e}\right)-\mathbf{r}_{j_{e}}\left(z_{e}\right)\right)^{2}\right) \\
& =\frac{1}{\mathcal{Z}_{1}} \int \mathcal{D} \Gamma \mathrm{e}^{-H_{\text {ev }}-H_{\text {align }}} \sum_{M=0}^{\infty} \frac{1}{M !} \\
& \times\left(\frac{\tilde{\mu}^{2}}{L} \sum_{i, j=1}^{N} \int_{0}^{L} \mathrm{~d} z \exp \left(-\frac{1}{2 a^{2}}\left(\mathbf{r}_{i}(z)-\mathbf{r}_{j}(z)\right)^{2}\right)\right)^{M+J} \\
& =\frac{\left(\tilde{\mu}^{2}\right)^{J}}{\mathcal{Z}_{1}}\left(\frac{\partial}{\partial \tilde{\mu}^{2}}\right)^{J} \int \mathcal{D} \Gamma \mathrm{e}^{-H_{\mathrm{ev}}-H_{\text {align }}} \\
& \times \exp \left(\frac{\tilde{\mu}^{2}}{L} \sum_{i, j=1}^{N} \int_{0}^{L} \mathrm{~d} z \exp \left(-\frac{1}{2 a^{2}}\left(\mathbf{r}_{i}(z)-\mathbf{r}_{j}(z)\right)^{2}\right)\right) .
\end{aligned}
$$

As we can see, the expression after the partial derivative is $\lim _{n \rightarrow 0} \mathcal{Z}_{n+1}=\mathcal{Z}_{1}$. Hence we get an analogous result to Eq. (B.109):

$$
\mathcal{M}_{J}=\frac{\left(\mu^{2}\right)^{J}}{\mathcal{Z}_{1}}\left(\frac{\partial}{\partial \mu^{2}}\right)^{J} \mathcal{Z}_{1}
$$

The general form of $\mathcal{Z}_{n+1}$ is given in Eq. (3.32a):

$$
\mathcal{Z}_{n+1}=\exp \left(-N f_{0}\right) \int \mathcal{D} \Omega \exp \left(-N f_{n+1}\{\Omega\}\right)
$$

Thereby the contribution $f_{0}$, given in Eq. (3.31b), becomes in the limit $n \rightarrow 0$ :

$$
f_{0} \stackrel{n \rightarrow 0}{=}-\frac{\mu^{2}}{2}+\frac{\lambda n_{0} L}{2} .
$$


The term $f_{n+1}$ is given in Eq. (3.33); in the limit $n \rightarrow 0$ the sums over the HRS vanishes:

$$
f_{1}\{\Omega\}=\lim _{n \rightarrow 0} f_{n+1}\{\Omega\}=0-\ln \mathfrak{z} \quad \text { with } \quad \mathfrak{z}=\int \mathcal{D} \hat{r}(z) \exp \left(-H_{\text {align, } 1}^{(n+1)}\{\hat{r}(z)\}\right)
$$

The Hamiltonian $H_{\text {align, } 1}^{(n+1)}\{\hat{r}(z)\}$ should not depend on the parameter $\mu^{2}$. Therefore $f_{1}\{\Omega\}$ is a constant when deriving with respect to $\mu^{2}$. With these expressions Eq. (C.91) becomes in the limit $n \rightarrow 0$ :

$$
\mathcal{Z}_{1}=\exp \left(\frac{N \mu^{2}}{2}+\frac{N L \lambda n_{0}}{2}+\text { const. }\right) \text {. }
$$

This form together with Eq. (C.90) yields the same result for the expectation values $\mathcal{M}_{J}$ as for the RLP model, Eq. (2.61):

$$
\mathcal{M}_{J}=[M(M-1) \cdots(M-J+1)]=\left(\frac{\mu^{2} N}{2}\right)^{J} .
$$

In particular, the mean cross-link density and the standard deviation are given by:

$$
\begin{aligned}
& \frac{[M]}{N}=\frac{\mu^{2}}{2} \\
& \Delta M=\sqrt{\left[M^{2}\right]-[M]^{2}}=\sqrt{\frac{\mu^{2} N}{2}}
\end{aligned}
$$

so that $\Delta M /[M] \propto N^{-1 / 2}$.

It is noteworthy that for these results we did not use any expressions which are only valid close to the sol-gel transition, so Eq. (C.96) holds for arbitrary $\mu^{2}$. If, however, density fluctuations were allowed during cross-linking, contributions from the one replica sector of $f_{n+1}\{\Omega\}$ in Eq. (3.32b) may change the average cross-link density. 
Appendix D

\section{Expressions for the Wet Granulates}

\section{D.1 Infinite Sums similar to the Exponential Function}

$$
\begin{gathered}
\sum_{r=0}^{\infty} \frac{x^{r}}{r !} r=\sum_{r=1}^{\infty} \frac{x^{r}}{r !} r=x \sum_{r=1}^{\infty} \frac{x^{r-1}}{(r-1) !}=x \sum_{r=0}^{\infty} \frac{x^{r}}{r !}=x \mathrm{e}^{x} \\
\sum_{r=0}^{\infty} \frac{x^{r}}{r !}(r-1)=\sum_{r=0}^{\infty} \frac{x^{r}}{r !} r-\sum_{r=0}^{\infty} \frac{x^{r}}{r !}=x \mathrm{e}^{x}-\mathrm{e}^{x}=(x-1) \mathrm{e}^{x}
\end{gathered}
$$

\section{D.2 Inverting the Equation $x^{-2} \mathrm{e}^{x}=t$}

In section 5.3.2, we derived an equation for the evolution of the inverse dimensionless temperature $x$ :

$$
x^{-2} \mathrm{e}^{x}\left(1+\mathcal{O}\left(x^{-1}\right)\right)=t,
$$

in the asymptotic limit $x, t \rightarrow \infty$. Here we solve this equation for $x$. We proceed in analogy to the inversion of a similar equation discussed in [de Bruijn, 1958, Chapter 2.4]. First, we take the logarithm on both sides of Eq. (D.3):

$$
\begin{aligned}
-2 \ln x+x+\ln \left(1+\mathcal{O}\left(x^{-1}\right)\right) & =\ln t \\
-2 \ln x+x+\mathcal{O}\left(x^{-1}\right) & =\tau,
\end{aligned}
$$

with $\tau:=\ln t$. Now, we transform this equation to

$$
x\left(1-2 \frac{\ln x}{x}+\mathcal{O}\left(x^{-2}\right)\right)=\tau,
$$

which yields two statements:

$$
\begin{aligned}
& \text { 1) } \lim _{\tau \rightarrow \infty}\left|\frac{x^{-1}}{\tau^{-1}}\right|=1<\infty \quad \Rightarrow \quad x^{-1}=\mathcal{O}\left(\tau^{-1}\right) \quad \text { and } \\
& \text { 2) } \lim _{\tau \rightarrow \infty}\left|\frac{\ln x}{\ln \tau}\right|=1<\infty \quad \Rightarrow \quad \ln x=\mathcal{O}(\ln \tau)
\end{aligned}
$$


Therefore in Eq. (D.4) we can replace $\mathcal{O}\left(\tau^{-1}\right)$ by $\mathcal{O}\left(x^{-1}\right)$ using Eq. (D.6a),

$$
x=\tau+2 \ln x+\mathcal{O}\left(\tau^{-1}\right)
$$

Furthermore we can plug the statement (D.6b) in the main equation (D.7) and successively obtain higher and higher orders of the solution $x(t)$ :

- Step 1: (D.6b) in (D.7)

$$
\begin{aligned}
x & =\tau+\mathcal{O}(\ln \tau)+\mathcal{O}\left(\mathcal{T}^{-1}\right)=\tau\left(1+\mathcal{O}\left(\frac{\ln \tau}{\tau}\right)\right) \\
\ln x & =\ln \tau+\mathcal{O}\left(\frac{\ln \tau}{\tau}\right)
\end{aligned}
$$

- Step 2: (D.8b) in (D.7)

$$
\begin{aligned}
x & =\tau+2 \ln \tau+\mathcal{O}\left(\frac{\ln \tau}{\tau}\right)+\mathcal{O}\left(\tau^{-1}\right) \\
& =\tau\left(1+2 \frac{\ln \tau}{\tau}\right)\left(1+\mathcal{O}\left(\frac{\ln \tau}{\tau(\tau+2 \ln \tau)}\right)\right) \\
\ln x & =\ln \tau+\ln \left(1+2 \frac{\ln \tau}{\tau}\right)+\mathcal{O}\left(\frac{\ln \tau}{\tau(\tau+2 \ln \tau)}\right) \\
& =\ln \tau+\frac{2 \ln \tau}{\tau}-\frac{1}{2}\left(\frac{2 \ln \tau}{\tau}\right)^{2}+\mathcal{O}\left(\frac{\ln \tau}{\tau}\right)^{3}+\mathcal{O}\left(\frac{\ln \tau}{\tau^{2}}\right)
\end{aligned}
$$

- Step 3: (D.9b) in (D.7)

$$
x=\tau+2 \ln \tau+\frac{4 \ln \tau}{\tau}-\left(\frac{2 \ln \mathcal{X}}{\tau}\right)^{2}+\mathcal{O}\left(\frac{\ln \tau}{\tau^{2}}\right)+\mathcal{O}\left(\tau^{-1}\right)
$$

Now, it would be possible to find an expression for $\ln x$ and plug it into Eq. (D.7), again. However that would yield additional terms of an order higher than $\mathcal{O}\left(\tau^{-1}\right)$, and thus could be absorbed by $\mathcal{O}\left(\tau^{-1}\right)$ in Eq. (D.7). Hence, the result

$$
x(t)=\ln t+2 \ln \ln t+\frac{4 \ln \ln t}{\ln t}+\mathcal{O}\left(\frac{1}{\ln t}\right)
$$

is the highest order for $x(t)$ that the original equation (D.3) can yield.

\section{D.3 Computation of the radial distribution function $g(r)$}

In Sec. 5.4.2.3 we consider the radial distribution function, which is the radial component of Eq. (5.45). Here, some details for the computation for a particle configuration $\left\{\mathbf{r}_{1}, \ldots, \mathbf{r}_{N}\right\}$ are given.

To compute $g(r)$ for small values of $r$ (i.e. $r \leq r^{*}=6.4 d$ ), the algorithm chooses one particle $\mathbf{p}_{1} \in\left\{\mathbf{r}_{1}, \ldots, \mathbf{r}_{N}\right\}$ and records the distance of all particles $\mathbf{p}_{2}$ closer than 
$r^{*}$. Repeating this procedure for all particles $\mathbf{p}_{1} \in\left\{\mathbf{r}_{1}, \ldots, \mathbf{r}_{N}\right\}$ yields the histogram of distances between pairs of particles, from which $g(r)$ can easily be computed by dividing with $4 \pi r^{2}$.

For increasing $r$, the number of pairs with distance $r$ increases rapidly and hence not all pairs of particles can be considered anymore; Therefore, for $r>r^{*}$, the algorithm chooses the particles $\mathbf{p}_{1}$ and $\mathbf{p}_{2}$ at random, with a distance of at least $r^{*}$; in repeating this procedure for about $10^{9}$ initial particles $\mathbf{p}_{1}$, the algorithm approximates the particle-distance histogram in a Monte-Carlo like fashion.

Two remarks have to be made about the system-boundaries: (i) For a pair of particles $\mathbf{p}_{1}$ and $\mathbf{p}_{2}$, only the closest distance compatible with the periodic boundary conditions (in $x$ - and $y$-direction) is considered. (ii) If particle $\mathbf{p}_{1}$ is close to a hard wall (in $z$-direction), then the probability to find a close-by particle is lower. Hence, whenever $g(r)$ is shown up to a distance $r=r_{\max }$, then particle $\mathbf{p}_{1}$ is chosen at least $r_{\max }$ away from the hard wall, in order to avoid the influence of the boundary. 



\section{Further Results from the Spider Silk Model}

\section{Contents}

E.1 Effect of the Continuous Background . . . . . . . 187

E.2 Relevance of the Coherent Part of the Scattering Function 187

E.3 Simplification of the Scattering Amplitude $A(\mathbf{q}) \ldots \ldots 188$

\section{E.1 Effect of the Continuous Background}

In this section we explain why it is necessary to include the continuous background between the crystallites, introduced in section 4.2.4.

Without the background, the system has vast, unphysical density fluctuations on the length scale of the crystallite distances, resulting in a large scattering function $G(\mathbf{q})$ for small $q$-values. As already explained in section 4.2.4, these density fluctuations are unphysical because the space between the crystallites is filled with the amorphous matrix and water molecules. Fig. E.1 shows the scattering profiles in $x y$-direction with and without the continuous background. As expected, the system without the background shows a large increase of the scattering function for small $q$-values. The continuous background, however, acts as a low-pass filter on the scattering density and therefore annihilates the large intensities for small $q$.

\section{E.2 Relevance of the Coherent Part of the Scattering Function}

Here we discuss the influence of the coherent part of the scattering function of Eq. (4.20). Fig. E.2 shows a comparison of the incoherent part

$$
G_{1}(\mathbf{q})=\int \mathcal{D} \underline{\underline{D}}\left|A\left(\underline{\underline{D}}^{T} \mathbf{q}\right)\right|^{2},
$$

which is used to calculate the scattering function throughout chapter 4 , and the contribution

$$
G_{2}^{\prime}(\mathbf{q}):=\left|\int \mathcal{D} \underline{\underline{D}} A\left(\underline{\underline{D}}^{T} \mathbf{q}\right)\right|^{2}
$$




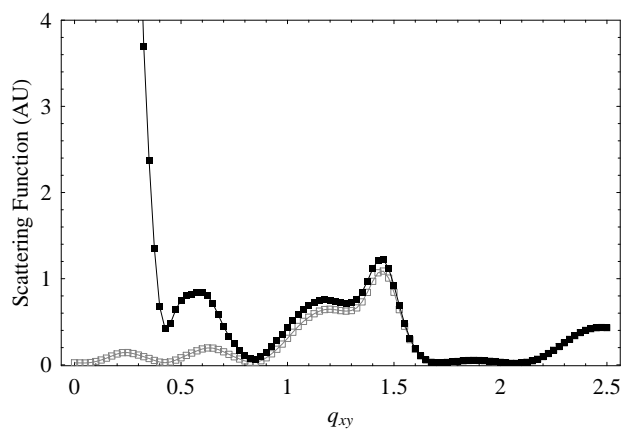

Figure E.1: Scattering function in $x y$-direction with $(\square)$ and without ( $\square)$ the continuous background. Without background, density fluctuations on large length scales cause an increase of the scattering function for small $q$-values.

of the coherent part, $G_{2}(\mathbf{q})=(S(\mathbf{q})-1)\left|\int \mathcal{D} \underline{\underline{D}} A\left(\underline{\underline{D}}^{T} \mathbf{q}\right)\right|^{2}$.

Neglecting the coherent part is plausible for two reasons. Firstly, because the contribution of $G_{2}^{\prime}(\mathbf{q})$ is small compared to the incoherent part $G_{1}(\mathbf{q})$, as seen in the figure. And secondly, the length scale for the distances between the crystallites is much larger than atom length scales investigated here. On the length scales we are interested in, we expect $S(\mathbf{q}) \approx 1$, assuming that the crystallite positions have no long range order. Therefore, the prefactor $(S(\mathbf{q})-1)$ additionally reduces the contribution of the coherent term.

The (002) peak is special for the coherent scattering term $G_{2}^{\prime}(\mathbf{q})$. All peaks except for the (002) peak have a very small contribution in $G_{2}^{\prime}(\mathbf{q})$ because of the white average of the crystallites' rotations about the fiber axis. This makes coherent scattering from different crystallites very unlikely, no matter how the crystallites are arranged in space. Since there is a preferential alignment of the crystallites in the $z$-direction, however, contributions of coherent scattering from different crystallites (which are contained in the term $G_{2}^{\prime}(\mathbf{q})$ ) are not completely destroyed; therefore, if the crystallites' distance in the $z$-direction is a multiple of the unit cell size $a_{z}$, causing a large contribution in the prefactor $(S(\mathbf{q})-1)$ at the position of the (002) peaks, a contribution of $G_{2}(\mathbf{q})$ will be present.

\section{E.3 Simplification of the Scattering Amplitude $A(\mathbf{q})$}

As defined in Eq. (4.16), the scattering amplitude of a single crystallite is given by

$$
A(\mathbf{q})=\sum_{\mathbf{m}=\mathbf{1}}^{\mathbf{M}} \sum_{k=1}^{K} F_{k}(\mathbf{q}) \exp \left(\mathrm{iq} \cdot \mathbf{r}_{\mathbf{m}, k}\right)
$$




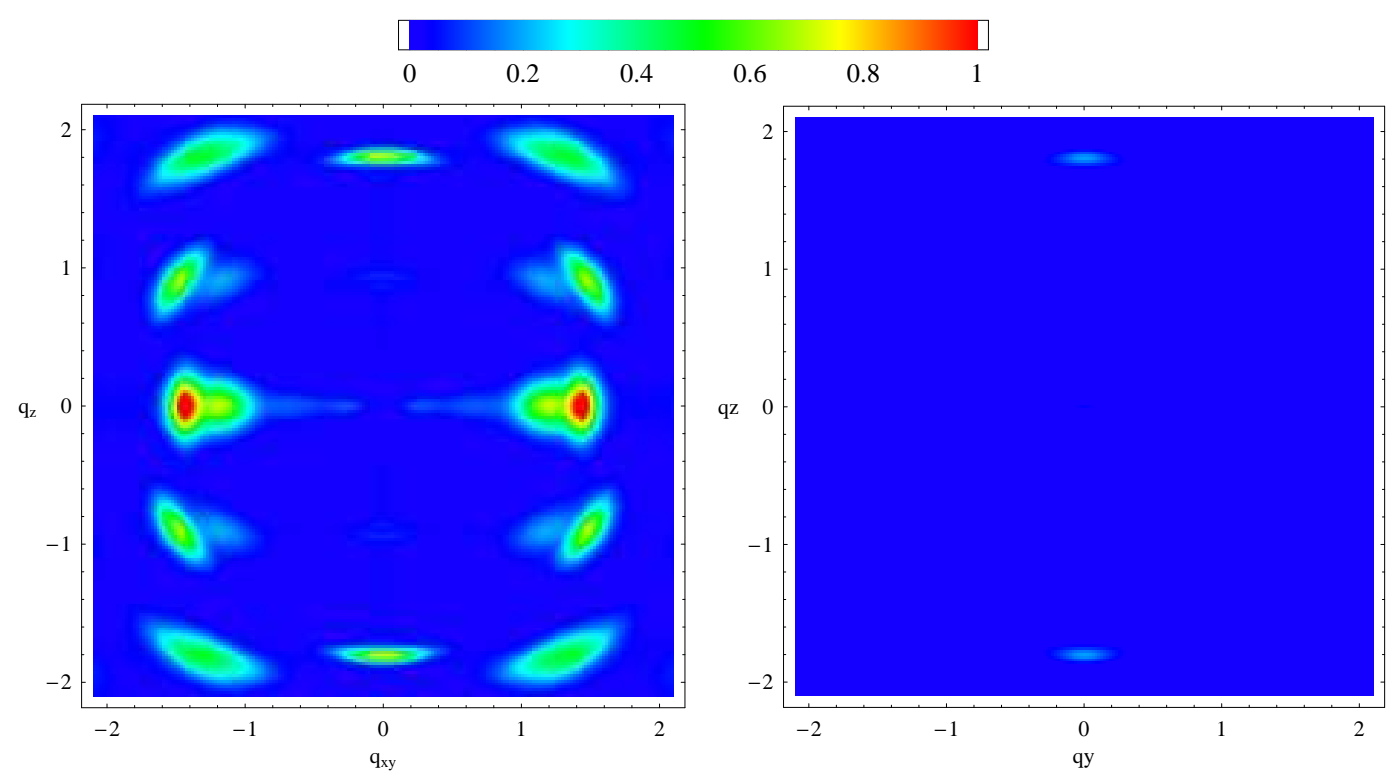

Figure E.2: Left: Calculated scattering images as in Fig. 4.12, but for a Gaussian distribution (rounded to integers) of the crystallite sizes $M_{x}, M_{y}$ and $M_{z}$. The widths are $\Delta M_{x}=2, \Delta M_{y}=0.75$ and $\Delta M_{z}=3$ respectively. Right: correlated part $G_{2}^{\prime}(\mathbf{q})$ of the scattering function Eq. (4.20) in comparison to the uncorrelated part on the left.

The sums over the unit cells $\mathbf{m}=\left(m_{x}, m_{y}, m_{z}\right)$ are:

$$
\begin{aligned}
A(\mathbf{q})= & \sum_{m_{x}=1}^{M_{x}} \sum_{m_{y}=1}^{M_{y}} \sum_{m_{z}=1}^{M_{z}} \sum_{k=1}^{K} F_{k}(\mathbf{q}) \exp \left(\mathrm{iq}\left(m_{x} \mathbf{a}_{x}+m_{y} \mathbf{a}_{y}+m_{z} \mathbf{a}_{z}-\mathbf{s}_{\mathrm{cm}}+\mathbf{r}_{k}\right)\right) \\
= & \sum_{m_{x}=1}^{M_{x}} \exp \left(\mathbf{i q} \mathbf{a}_{x} m_{x}\right) \sum_{m_{y}=1}^{M_{y}} \exp \left(\mathbf{i q} \mathbf{a}_{y} m_{y}\right) \sum_{m_{z}=1}^{M_{z}} \exp \left(\mathrm{iq} \mathbf{a}_{z} m_{z}\right) \\
& \times \exp \left(-\mathbf{i q} \cdot \frac{\left(M_{x}+1\right) \mathbf{a}_{x}+\left(M_{y}+1\right) \mathbf{a}_{y}+\left(M_{z}+1\right) \mathbf{a}_{z}}{2}\right) \sum_{k=1}^{K} F_{k}(\mathbf{q}) \exp \left(\mathbf{i q r} \mathbf{r}_{k}\right)
\end{aligned}
$$

For each $\nu=x, y, z$ the sum over $m_{\nu}$ is a geometric progression

$$
\sum_{m_{\nu}=1}^{M_{\nu}} \exp \left(\mathbf{i q a} \mathbf{a}_{\nu} m_{\nu}\right)=\frac{\exp \left(\mathbf{i q} \mathbf{a}_{\nu}\left(M_{\nu}+1\right)\right)-\exp \left(\mathbf{i q} \mathbf{a}_{\nu}\right)}{\exp \left(\mathbf{i q} \mathbf{a}_{\nu}\right)-1}
$$

and hence

$$
\begin{aligned}
\sum_{m_{\nu}=1}^{M_{\nu}} \exp \left(\mathbf{i q a} \mathbf{a}_{\nu} m_{\nu}\right) \exp \left(-\mathbf{i q} \cdot \frac{\left(M_{\nu}+1\right) \mathbf{a}_{\nu}}{2}\right) \\
=\frac{\exp \left(\mathbf{i q} \mathbf{a}_{\nu}\left(M_{\nu}+1\right)\right)-\exp \left(\mathbf{i q} \mathbf{a}_{\nu}\right)}{\exp \left(\mathbf{i q} \mathbf{a}_{\nu}\right)-1} \frac{\exp \left(-\mathbf{i q} \mathbf{a}_{\nu}\left(M_{\nu}+2\right) / 2\right)}{\exp \left(-\mathbf{i q} \mathbf{a}_{\nu} / 2\right)}
\end{aligned}
$$


190 APPENDIX E. FURTHER RESULTS FROM THE SPIDER SILK MODEL

$$
\begin{aligned}
& =\frac{\exp \left(\mathbf{i q} \mathbf{a}_{\nu} M_{\nu} / 2\right)-\exp \left(-\mathbf{i q} \mathbf{a}_{\nu} M_{\nu} / 2\right)}{\exp \left(\mathbf{i q} \mathbf{a}_{\nu} / 2\right)-\exp \left(-\mathbf{i q} \mathbf{a}_{\nu} / 2\right)} \\
& =\frac{\sin \left(\mathbf{i q} \mathbf{a}_{\nu} M_{\nu} / 2\right)}{\sin \left(\mathbf{i q} \mathbf{a}_{\nu} / 2\right)}
\end{aligned}
$$

With that, Eq. (E.4) becomes:

$$
A(\mathbf{q})=L_{M_{x}}\left(\mathbf{q} \mathbf{a}_{x}\right) L_{M_{y}}\left(\mathbf{q} \mathbf{a}_{y}\right) L_{M_{z}}\left(\mathbf{q} \mathbf{a}_{z}\right) \sum_{k=1}^{K} F_{k}(\mathbf{q}) \exp \left(\mathbf{i} \mathbf{q} \mathbf{r}_{k}\right),
$$

where $L_{M_{\nu}}\left(\mathbf{q} \mathbf{a}_{\nu}\right)=\frac{\sin \left(\mathbf{q} \mathbf{a}_{\nu} M_{\nu} / 2\right)}{\sin \left(\mathbf{q} \mathbf{a}_{\nu} / 2\right)}$ is the Laue function. The result (E.7) is used in Sec. 4.3.3. 
APPENDIX F

\section{Used Symbols and Notation}

\section{Contents}

F.1 Notation . . . . . . . . . . . . . . . . . . . . 191

F.2 Symbols for the Randomly Cross-Linked Particle Model . . 192

F.3 Symbols for the Cross-Linked Directed Polymers . . . . . 194

F.4 Symbols for the Spider Silk Model . . . . . . . . . . . 197

F.5 Symbols for the Wet Granular System . . . . . . . . . 199

\section{F.1 Notation}

A few matters concerning the notation are addressed here. The following remarks apply to the whole thesis, in the attempt to allow for a simple notation, but still dispel possible confusion:

For volume integrals with a $D$-dimensional variable $\mathbf{x}$, the simplified notation $\int_{V} \mathrm{~d} \mathbf{x} \ldots$ or $\int \mathrm{d} \mathbf{x} \ldots$ is used, instead of $\int_{V} \mathrm{~d}^{D} \mathbf{x} \ldots$ which can appear quite overloaded as soon as expressions become more complicated. Confusion with line integrals, like $\int \mathbf{F}(\mathbf{x}) \mathrm{d} \mathbf{x}:=\int \mathbf{F}(\mathbf{x}(t)) \cdot \mathbf{x}^{\prime}(t) \mathrm{d} t$, is not given, because they do not appear in this work. Fourier and real functions have the same name; e.g. $U(\mathbf{q})$ is the Fourier transform of the potential $U(\mathbf{r})$ in chapters 2 and 3. The type of the argument real-space variable $\mathbf{r}$ with units of length, or Fourier-space variable $\mathbf{q}$ with units of inverse length - determines what function is meant. To describe the asymptotic behavior of a function, the Landau symbol $\mathcal{O}$ is used. And the imaginary unit is always a roman " $\mathrm{i}$ " to avoid confusion with a variable " $i$ ".

In the following, the used symbols and variables for the main chapters of this thesis can be found. For most symbols, also a reference to its introduction or definition is listed. "Temporary symbols", however, which are only used near their definition, are not quoted in order to keep the list short and clear. 


\section{F.2 Symbols for the Randomly Cross-Linked Particle Model}

$[\cdot] \quad$ disorder average, see Sec. 2.3 and Eq. (2.7)

$\int_{\xi_{1}^{2}, \ldots, \xi_{r}^{2}}=\int_{0}^{\infty} \mathrm{d} \xi_{1}^{2} \mathcal{P}\left(\xi_{1}^{2}\right) \cdots \mathrm{d} \xi_{r}^{2} \mathcal{P}\left(\xi_{r}^{2}\right)$, introduced in Eq. (B.53)

$\|, \perp \quad$ used for the separation of replicated $D(n+1)$-dimensional vectors into "common" and "relative" components; see Eqs. (2.35)-(2.37)

ORS $\quad$ zero replica sector, $0 \mathrm{RS}=\{\hat{0}\}$, see Sec. 2.4.3

1RS one replica sector, set of $\hat{q}$-values with exactly one replica component non-zero: $1 \mathrm{RS}=\left\{\hat{q} \neq \hat{0} \mid \hat{q}=\left(\mathbf{0}, \ldots, \mathbf{0}, \mathbf{q}^{(\alpha)}, \mathbf{0}, \ldots, \mathbf{0}\right)\right.$ with $\left.0 \leq \alpha \leq n\right\}$, see Sec. 2.4.3

a typical cross-link distance (mostly appearing as $a^{2}$ )

$\mathcal{A} \quad$ arbitrary and unspecified quantity, used to define general expressions like a thermal average

$\alpha \quad$ specifying the replica and appearing as index ${ }^{(\alpha)} ; \alpha \in\{0, \ldots, n\}$

$C_{\gamma} \quad=\left(a^{2}+\xi_{\gamma}^{2}\right)^{-1}$ defined in Eq. (B.54)

$C \quad=\sum_{\gamma=1}^{r} C_{\gamma}$ defined in Eq. (B.54)

$\mathcal{C} \quad$ cross-link configuration, representing the $M$ pairs of connected particles: $\mathcal{C}=\left\{i_{e}, j_{e}\right\}_{e=1}^{M}$

D spatial dimension

$\mathrm{Dr} \quad=\mathrm{d} \mathbf{r} / V$ dimensionless measure, introduced in the partition function Eq. (2.6)

$\mathrm{Dx}_{\|} \quad=\mathrm{d} \mathbf{x}_{\|} / V_{\|}$dimensionless measure for $\|$-vectors, residing in the volume $V_{\|}$

$\mathrm{D} \hat{r}=\mathrm{d} \hat{r} / V^{n+1}$ dimensionless measure for "hatted" vectors

$\mathcal{D} \Omega \quad$ measure for the integration over the fluctuating field $\Omega$; $\mathcal{D} \Omega \propto \prod_{\hat{q}} \mathrm{~d} \Omega(\hat{q})$, see Eq. (B.16)

$\Delta(\hat{q}) \quad=\exp \left(-\hat{q}^{2} a^{2} / 2\right)$, introduced in Eq. (2.20)

$\varepsilon \quad$ distance from the critical cross-link concentration, $\varepsilon=\mu^{2}-1$

$f_{0} \quad=-\phi^{n} \frac{\mu^{2}}{2}+(n+1) \frac{\lambda n_{0}}{2}$, see Eq. (2.19)

$\tilde{f}_{n+1}\left\{\hat{r}_{j}\right\} \quad$ defined such that $\mathcal{Z}_{n+1}=\int\left(\prod_{j} \mathrm{~d} \hat{r}_{j}\right) \exp \left(-N \tilde{f}_{n+1}\left\{\hat{r}_{j}\right\}\right)$, see Eq. (2.14)

$f_{n+1}\{\Omega\} \quad$ defined such that $\mathcal{Z}_{n+1}=\mathrm{e}^{-N f_{0}} \int \mathcal{D} \Omega \exp \left(-N f_{n+1}\{\Omega\}\right)$, see Eqs. (2.27)

$F_{\mathcal{C}} \quad=-\ln Z_{\mathcal{C}}$, free energy (all energies in units of $k_{\mathrm{B}} T$ )

$\phi \quad=(2 \pi)^{D / 2} \cdot a^{D} / V$

$G \quad$ shear modulus; see Eqs. (2.53) and Sec. 2.9.3

$H_{\mathrm{C}} \quad=H_{\mathrm{Xlink}}+H_{\mathrm{ev}}$

$H_{\mathrm{ev}} \quad$ excluded volume Hamiltonian, $H_{\mathrm{ev}}\left(\left\{\mathbf{r}_{j}\right\}\right)=\frac{\lambda}{2} \sum_{i, j=1}^{N} U\left(\mathbf{r}_{i}-\mathbf{r}_{j}\right)$

$H_{\mathrm{ev}}^{(n+1)}=\sum_{\alpha=0}^{n} H_{\mathrm{ev}}\left(\left\{\mathbf{r}_{j}^{(\alpha)}\right\}\right)$, replicated excluded-volume interaction

$H_{\text {Xlink }} \quad$ cross-link Hamiltonian, $H_{\text {Xlink }}=\frac{1}{2 a^{2}} \sum_{e=1}^{M}\left(\mathbf{r}_{i_{e}}-\mathbf{r}_{j_{e}}\right)^{2}$, see Eq. (2.1)

HRS higher replica sector, all $\hat{q}$-vectors with at least two non-zero entries: $\mathrm{HRS}=\{\hat{q} \mid \hat{q} \neq \hat{0} \vee \hat{q} \notin 1 \mathrm{RS}\}$, see Sec. 2.4.3

$\lambda \quad$ strength of the excluded-volume interaction, see $H_{\mathrm{ev}}$

$\tilde{\lambda}(\hat{q}) \quad=n_{0} \lambda U(\hat{q})-\phi^{n} \mu^{2} \Delta(\hat{q})$, introduced in Eq. (2.24b). 
$M \quad$ number of cross-links

$\mu^{2} \quad$ parameter of the Deam-Edwards distribution, see Eq. (2.7); found to be the average coordination number of the particles, see Sec. 2.9.1

$\tilde{\mu}^{2} \quad=\frac{V}{2 N a^{D}} \frac{\mu^{2}}{(2 \pi)^{D / 2}}=\frac{\mu^{2}}{2 N \phi}$

$N \quad$ number of particles

$n \quad$ number of replicas

$n_{0} \quad=N / V$, particle density

$\mathrm{O}(\hat{x}) \quad=\frac{1}{N} \sum_{j=1}^{N} \delta\left(\hat{x}-\hat{r}_{j}\right)$, replicated real-space density; see Eq. (2.16)

$\mathrm{O}(\hat{q}) \quad=\frac{1}{N} \sum_{j=1}^{N} \exp \left(\mathrm{i} \hat{q} \hat{r}_{j}\right)$, replicated Fourier-space density; see Eq. (2.17a)

$\Omega(\hat{q}) \quad$ order parameter of the theory, see e.g. Eq. (2.27b); in the thermodynamic limit equal to $\mathrm{O}(\hat{q})$

$\bar{\Omega}_{\mathrm{sp}} \quad$ saddle-point solution for the order parameter; see Eqs. (2.33) and (2.48)

$\bar{\Omega}_{u}(\hat{q}) \quad$ Ansatz for the order parameter with displacement field $u_{\perp}\left(\mathbf{x}_{\|}\right)$, see Eq. (2.45)

$P(\mathcal{C}) \quad$ probability for the cross-link configuration $\mathcal{C}$; see Sec. 2.3

$\mathcal{P}\left(\xi^{2}\right) \quad$ distribution of localization lengths; see Eq. (2.43)

* $\quad \pi /(2 \pi)=1 / 2$; simplifying notation

$Q \quad$ gel fraction, i.e. the fraction of particles in the macroscopic cluster; introduction in Sec. 2.6 and discussion in Sec. 2.9.2

$u_{\perp}\left(\mathbf{x}_{\|}\right) \quad$ spatially dependent deformation field; see Sec. 2.6.2

$\mathbf{u}_{\mathrm{phys}}^{(\alpha)}\left(\mathbf{x}_{\|}\right)$physically relevant deformation field (relative to the state during crosslinking); see Sec. 2.6.3

$U(\mathbf{r}) \quad$ potential for the excluded-volume interaction $H_{\mathrm{ev}}$

$U(\mathbf{q}) \quad$ Fourier transform of the potential $U(\mathbf{r})$ for the excluded-volume interaction $H_{\mathrm{ev}}$

$U(\hat{q}) \quad=U\left(\mathbf{q}^{(\alpha)}\right)$ for $\hat{q}=\left(\mathbf{0}, \ldots, \mathbf{0}, \mathbf{q}^{(\alpha)}, \mathbf{0}, \ldots, \mathbf{0}\right) \in 1 \mathrm{RS}$

$\mathbf{r}_{j} \quad$ position of particle $j$

$V \quad D$-dimensional volume of the system

$V_{\|} \quad=V(n+1)^{D / 2}$; rescaled volume of the system for $\|$-vectors, which are scaled by a factor of $\sqrt{n+1}$, compared to normal vectors; see Sec. 2.6.1

$\xi \quad$ localization length, quantifying the extent of the fluctuations of a localized particle; see Sec. 2.6

$\Xi_{r, a^{2}} \quad=\int_{\xi_{1}^{2}, \ldots, \xi_{r}^{2}} \ln \left(\left(\frac{V}{(2 \pi)^{D / 2}}\right)^{r-1}\left(\frac{\prod_{\gamma=1}^{r}\left(a^{2}+\xi_{\gamma}^{2}\right)^{-1}}{\sum_{\gamma=1}^{r}\left(a^{2}+\xi_{\gamma}^{2}\right)^{-1}}\right)^{D / 2}\right)$

$Z_{\mathcal{C}} \quad$ partition function, depending on the cross-link configuration $\mathcal{C}$

$\mathcal{Z}_{n+1} \quad$ Made such that $\left[Z_{\mathcal{C}}^{n}\right]=\mathcal{Z}_{n+1} / \mathcal{Z}_{1}$, actual form given in Eq. (2.14c)

$\mathfrak{z} \quad$ effective one-particle partition function, see Eq. $(2.27 \mathrm{c})$ 


\section{F.3 Symbols for the Cross-Linked Directed Polymers}

$[\cdot] \quad$ disorder average, see Sec. 3.3.2 and Eq. (3.22)

$\int_{\xi^{2}, z}=\int_{0}^{\infty} \mathrm{d} \xi^{2} \mathcal{P}\left(\xi^{2}, z\right)$, introduced in Eq. (3.45)

$\|, \perp \quad$ used for the separation of replicated $D(n+1)$-dimensional vectors into "common" and "relative" components; see Appendix A

$\sum_{\hat{q} \in \mathrm{HRS}}^{\prime} \quad \sum_{\hat{q} \in \mathrm{HRS}}^{\prime}(\ldots):=Q+\sum_{\hat{q} \in \mathrm{HRS}}(\ldots)$; simplifying definition, see Eq. (3.37)

ORS $\quad$ zero replica sector, $0 \mathrm{RS}=\{\hat{0}\}$, see Sec. 3.4.2

1RS one replica sector, set of $\hat{q}$-values with exactly one replica component non-zero: $1 \mathrm{RS}=\left\{\hat{q} \neq \hat{0} \mid \hat{q}=\left(\mathbf{0}, \ldots, \mathbf{0}, \mathbf{q}^{(\alpha)}, \mathbf{0}, \ldots, \mathbf{0}\right)\right.$ with $\left.0 \leq \alpha \leq n\right\}$, see Sec. 3.4.2

$a$ typical cross-linking length in the transverse plane; see $H_{\text {Xlink }}$ and Eq. (3.15)

$a_{\ell}^{2} \quad=a^{2} / \ell^{2}$, defined in Eq. (3.59b)

$A \quad D$-dimensional (hyper-)area of the system in the transverse plane; see Sec. 3.3

$\mathcal{A} \quad$ arbitrary and unspecified quantity, used to define general expressions like a thermal average

$\alpha \quad$ specifying the replica and appearing as index ${ }^{(\alpha)} ; \alpha \in\{0, \ldots, n\}$

$\mathcal{C} \quad$ cross-link configuration, representing the $M$ pairs of chains connected at height $z_{e} ; \mathcal{C}:=\left\{\left(i_{e}, j_{e}, z_{e}\right)\right\}_{e=1}^{M}$; see Sec. 3.3

$\mathcal{D} \mathbf{r}(z) \quad$ measure for the functional integral over all possible conformations of a chain $\mathbf{r}(z)$; see Eq. (3.21)

$\Delta(\hat{q}) \quad=\exp \left(-\hat{q}^{2} a^{2} / 2\right)$, introduced in Eq. (3.31c)

$\varepsilon \quad$ distance from the critical cross-link concentration, $\varepsilon=\mu^{2}-1$

$f \quad \frac{2}{3}+\frac{a^{2}}{\ell^{2}}$, see Eq. (3.60b)

$f_{0} \quad=-\phi^{n} \frac{\mu^{2}}{2}+(n+1) \frac{\lambda n_{0} L}{2}$; see Eq. (3.31b)

$\tilde{f}_{n+1}\left\{\hat{r}_{j}\right\} \quad$ defined such that $\mathcal{Z}_{n+1}=\int\left(\prod_{j=1}^{N} \mathcal{D} \hat{r}_{j}(z)\right) \exp \left(-N \tilde{f}_{n+1}\left\{\hat{r}_{j}\right\}\right)$; see Eq. (3.27)

$f_{n+1}\{\Omega\} \quad$ defined such that $\mathcal{Z}_{n+1}=\mathrm{e}^{-N f_{0}} \int \mathcal{D} \Omega \exp \left(-N f_{n+1}\{\Omega\}\right)$, see Eqs. (3.32) $\phi \quad=(2 \pi)^{D / 2} \cdot a^{D} / A$

$H_{\text {align }}=\frac{\sigma}{2} \sum_{j=1}^{N} \int_{0}^{L} \mathrm{~d} z \dot{\mathbf{r}}_{j}^{2}(z)$; alignment interaction of the whole system; defined in Eq. (3.17); note that $H_{\text {align }}=\sum_{j=1}^{N} H_{\text {align }, 1}\left\{\mathbf{r}_{j}\right\}$;

$H_{\text {align }, 1}=\frac{\sigma}{2} \int_{0}^{L} \mathrm{~d} z \dot{\mathbf{r}}^{2}(z)$; alignment interaction of a single chain $\mathbf{r}(z)$; see Sec. 3.2.1

$H_{\text {align }}^{(n+1)} \quad$ replicated alignment interaction; $H_{\text {align }}^{(n+1)}\left\{\hat{r}_{j}\right\}=\sum_{\alpha=0}^{n} H_{\text {align }}\left\{\mathbf{r}_{j}^{(\alpha)}\right\}$

$H_{\mathrm{ev}} \quad=\frac{\lambda}{2} \sum_{i, j=1}^{N} \int_{0}^{L} \mathrm{~d} z U\left(\mathbf{r}_{i}(z)-\mathbf{r}_{j}(z)\right)$; excluded volume interaction, defined in Eq. (3.16)

$H_{\mathrm{ev}}^{(n+1)} \quad$ replicated excluded volume interaction; $H_{\mathrm{ev}}^{(n+1)}\left\{\hat{r}_{j}\right\}=\sum_{\alpha=0}^{n} H_{\mathrm{ev}}\left\{\mathbf{r}_{j}^{(\alpha)}\right\}$

$H_{\text {Xlink }}=\frac{1}{2 a^{2}} \sum_{e=1}^{M}\left(\mathbf{r}_{i_{e}}\left(z_{e}\right)-\mathbf{r}_{j_{e}}\left(z_{e}\right)\right)^{2}$, defined in Eq. (3.15)

HRS higher replica sector, all $\hat{q}$-vectors with at least two non-zero entries: 
$\mathrm{HRS}=\{\hat{q} \mid \hat{q} \neq \hat{0} \vee \hat{q} \notin 1 \mathrm{RS}\}$, see Sec. 3.4 .2

$I\left(\hat{q}_{0}, z_{0}\right) \quad$ simplifying definition, actual form given in Eq. (3.36)

$L \quad$ height of the system in the direction of preferred alignment; see Sec. 3.3

$\ell^{2} \quad:=L /(2 \sigma) \propto R_{\mathrm{g}}^{2}$; simplifying definition, see Sec. 3.2.1 and Eq. (3.6)

$\lambda \quad$ strength of the excluded-volume interaction, see $H_{\mathrm{ev}}$ and def. (3.16)

$\tilde{\lambda}(\hat{q}) \quad=\lambda n_{0} U(\hat{q})-\phi^{n} \frac{\mu^{2}}{L} \Delta(\hat{q})$, introduced in Eq. (3.31d)

$M \quad$ number of cross-links

$\mu^{2} \quad$ parameter of the Deam-Edwards distribution, see Eq. (3.22); found to be the average coordination number of the chains; see Appendix C.7

$\tilde{\mu}^{2} \quad=\frac{\mu^{2}}{2 N \phi}=\frac{A}{2 N a^{D}} \frac{\mu^{2}}{(2 \pi)^{D / 2}}$

$n$ number of replicas

$N \quad$ number of chains

$\mathrm{O}(\hat{x}, z)=\frac{1}{N} \sum_{j=1}^{N} \delta\left(\hat{x}-\hat{r}_{j}(z)\right)$, replicated real-space density; see Eq. (3.29a)

$\mathrm{O}(\hat{q}, z)=\frac{1}{N} \sum_{j=1}^{N} \exp \left(\mathrm{i} \hat{q} \hat{r}_{j}(z)\right)$, replicated Fourier density; see Eq. (3.29b)

$\Omega(\hat{q}, z) \quad$ order parameter of the theory, see e.g. Eq. (3.32); in the thermodynamic limit equal to $\mathrm{O}(\hat{q}, z)$

$\bar{\Omega}(\hat{q}, z) \quad$ Ansatz for the order parameter $\Omega(\hat{q}, z)$, shown to solve the saddle-point equation; actual form given in Eq. (3.44)

$P(\mathcal{C}) \quad$ probability for the cross-link configuration $\mathcal{C}$; see Sec. 3.3.2

$\mathcal{P}\left(\xi^{2}, z\right) \quad$ distribution of localization lengths at a given height $z$; see Eq. (3.42)

$\mathcal{P}\left(\xi^{2}, s\right) \quad$ distribution of localization lengths at normalized height $s=z / L$; see Eq. (3.53)

$\pi(\theta, s) \quad$ distribution for $\theta$ at normalized system height $s=z / L$; see Eq. (3.60c)

$\bar{\pi}(\theta) \quad$ distribution for $\theta$, averaged over the system height; see Eq. (3.62)

$\delta \pi(\theta, s) \quad$ deviation of $\pi(\theta, s)$ from the mean value $\bar{\pi}(\theta)$, normalized with $\varepsilon$; see Eq. (3.64)

$q_{0 \ell \perp}^{2} \quad=\ell^{2} q_{0 \perp}^{2}$, defined in Eq. (3.59c)

$Q \quad$ gel fraction, i.e. the fraction of chains in the macroscopic cluster; introduction in Sec. 3.5 and discussion in Sec. 3.6.2

$R_{\mathrm{g}} \quad$ transverse radius of gyration of an uncrosslinked chain; $R_{\mathrm{g}}^{2}=\frac{D}{6} \frac{L}{2 \sigma}$, see Sec. 3.2 .1

$\theta \quad=f /\left(\varepsilon \xi_{\ell}^{2}\right)$; normalized inverse and squared localization length, see Sec. 3.7.1 and Eq. (3.60a)

$U(\mathbf{r}) \quad$ potential for the excluded-volume interaction $H_{\mathrm{ev}}$

$U(\mathbf{q}) \quad$ Fourier transform of the potential $U(\mathbf{r})$ for the excluded-volume interaction $H_{\mathrm{ev}}$

$U(\hat{q}) \quad=U\left(\mathbf{q}^{(\alpha)}\right)$ for $\hat{q}=\left(\mathbf{0}, \ldots, \mathbf{0}, \mathbf{q}^{(\alpha)}, \mathbf{0}, \ldots, \mathbf{0}\right) \in 1 \mathrm{RS}$

$\xi \quad$ localization length, quantifying the extent of the fluctuations of a localized chain segment; see Sec. 3.5

$\tilde{\xi}^{2} \quad=\xi^{2}+a^{2}$, defined in Eq. (3.49)

$\xi_{\ell}^{2} \quad=\xi^{2} / \ell^{2}$, defined in Eq. (3.59a)

$Z_{\mathcal{C}} \quad$ partition function, depending on the cross-link configuration $\mathcal{C}$, introduced in Eq. (3.20) 
$\mathcal{Z}_{n+1} \quad$ Made such that $\left[Z_{\mathcal{C}}^{n}\right]=\mathcal{Z}_{n+1} / \mathcal{Z}_{1}$, actual form given in Eq. (3.27)

$\mathfrak{z} \quad$ effective one-particle partition function, see Eq. (3.32c) 


\section{F.4 Symbols for the Spider Silk Model}

\langle\rangle$\quad$ average over crystallite positions $\mathbf{R}^{(j)}$ and orientations $\underline{\underline{D}}^{(j)}$; see Sec. 4.2.3 and Eq. (4.6)

$\sum_{\mathbf{m = 1}}^{\mathbf{M}}=\sum_{m_{x}=1}^{M_{x}} \sum_{m_{y}=1}^{M_{y}} \sum_{m_{z}=1}^{M_{z}}$, simplifying notation

$\mathbf{a}_{x, y, z} \quad$ primitive vectors of the crystallite, see Sec. 4.2.2

$a_{x, y, z} \quad$ magnitudes of the primitive vectors $\mathbf{a}_{x}, \mathbf{a}_{y}$ and $\mathbf{a}_{z}$

$A(\mathbf{q}) \quad$ scattering amplitude of a single crystallite; defined in Eq. (4.16), treated in Sec. 4.3.3

$\mathrm{C}_{\alpha}$ for the alanine amino acid, the C-atom to which the $\mathrm{CH}_{3}$ group is bound

$\Delta y_{12} \quad$ amount of the shift of strands 1 and 2 (see Fig. 4.8) in $y$-direction

$\Delta z_{12}$ amount of the shift of strands 1 and 2 (see Fig. 4.8) in $z$-direction

$\Delta z_{24} \quad$ amount of the shift of strands 2 and 4 (see Fig. 4.8) in $z$-direction

$\underline{D}^{(j)} \quad$ rotation matrix specifying orientation of crystallite $j$; comprised by the Euler angles $\phi^{(j)}, \theta^{(j)}, \psi^{(j)}$; see Sec. 4.2.3 and Fig. 4.5

$\mathcal{D} \underline{\underline{D}} \quad$ measure for the integration over the orientation matrix $\underline{\underline{D}}$, see Sec. 4.2 .3 and Eq. (4.7)

$f_{k} \quad$ form factor of atom $k \in\{1, \ldots, K\}$, see Sec. 4.2.1

$\bar{f} \quad$ amplitude of the cavities $V(\mathbf{r})$, see Sec. 4.2.4 and Eq. (4.8)

$F_{k}(\mathbf{q}) \quad=f_{k}-\tilde{V}(\mathbf{q})$, effective form factor of atom $k$; see Eq. (4.12) and Sec. 4.3

$G(\mathbf{q}) \quad$ scattering function, general definition in Eq. (4.1), applied to our model in Eq. (4.11)

$G_{1}(\mathbf{q})$ incoherent contribution of the scattering function $G(\mathbf{q})$, accounting for scattering from a single crystallite; treated in Sec. 4.3.1

$G_{2}(\mathbf{q}) \quad$ coherent contribution of the scattering function $G(\mathbf{q})$, accounting for scattering from different crystallites; treated in Sec. 4.3.2

$K \quad$ number of atoms in one unit cell

$\mathbf{m}=\left(m_{x}, m_{y}, m_{z}\right)$; vector index specifying a unit cell inside a crystallite, see Sec. 4.2 .2

$M_{x, y, z}$ number of repetitions of the unit cell in $x$-, $y$ - and $z$-direction, respectively, in order to build one crystallite; see Sec. 4.2 .2

$N \quad$ number of crystallites

$\mathcal{P}_{\text {pos }} \quad \mathcal{P}_{\text {pos }}\left(\mathbf{R}^{(1)}, \ldots, \mathbf{R}^{(N)}\right)$ is the (correlated) distribution function for the crystallite positions $\mathbf{R}^{(1)}, \ldots, \mathbf{R}^{(N)}$; introduced in Sec. 4.2 .3

$\mathcal{P}_{\text {angle }} \mathcal{P}_{\text {angle }}(\phi, \theta, \psi)$ is the probability to find a crystallite orientation specified by the Euler angles $\phi, \theta, \psi$; see Sec. 4.2 .3 and Fig. 4.5

$\left.\begin{array}{l}\phi^{(j)} \\ \psi^{(j)}\end{array}\right\}$ see $\underline{\underline{D}}^{(j)}$

$q_{x y} \quad$ component of the wave vector $\mathbf{q}$ in $x$ - or $y$-direction; the two directions are indistinguishable due to rotational symmetry of the system about the $z$-axis

$\mathbf{R}^{(j)} \quad$ position of crystallite $j$ 
$\mathbf{r}_{k} \quad$ position of atom $k \in\{1, \ldots, K\}$ relative to the center of the unit cell

$\mathbf{r}_{\mathbf{m}, k} \quad$ position of atom $k$ of unit cell $\mathbf{m}$, relative to the center of the crystallite; see Eq. (4.4) and Sec. 4.2.2

$\mathbf{r}_{\mathbf{m}, k}^{(j)} \quad$ position of atom $k$ of unit cell $\mathbf{m}$ of crystallite $j$; see Eq. (4.5) and Sec. 4.2 .3

$\varrho_{0} \quad$ scattering strength of the continuous background between the crystallites, see Sec. 4.2.4

$\mathbf{s}_{\mathbf{m}} \quad$ vector pointing from the center of an unrotated crystallite to the center of unit cell $\mathbf{m}=\left(m_{x}, m_{y}, m_{z}\right)$, see Sec. 4.2.2

$\theta^{(j)} \quad$ Euler angle specifying the tilt of crystallite $j$ from the fiber axis, see $\underline{\underline{D}}^{(j)}$

$\theta_{0} \quad$ width of the distribution for the tilting angle $\theta$ of the crystallites from the fiber axis; see Sec. 4.2.3

$\left\langle u^{2}\right\rangle \quad$ mean square displacement of the atoms in any direction, to account for the Debye-Waller factor; see Eq. (4.21)

$V \quad$ volume of the system

$V(\mathbf{r}) \quad$ shape of the cavity of the "continuous background" around the atoms that prevents background scattering inside the crystallites; see Sec. 4.2.4 and Eq. (4.8)

$\tilde{V}(\mathbf{q}) \quad$ Fourier transform of the cavity $V(\mathbf{r})$

$\xi \quad$ width of the cavities $V(\mathbf{r})$ 


\section{F.5 Symbols for the Wet Granular System}

$\beta_{2} \quad$ kurtosis, quantifying deviations from a Gaussian velocity profile; see Eqs. (5.25)-(5.26)

d particle diameter

$d_{\mathrm{c}} \quad$ bond breaking distance, $d_{\mathrm{c}}=1.07 d$ unless noted otherwise

D spatial dimension

$D_{\mathrm{f}} \quad$ fractal dimension of a cluster

$\Delta E \quad$ bond breaking energy, i.e. energy needed for one bond rupture

$f_{\text {coll }} \quad$ collision frequency

$g(\mathbf{r}) \quad$ pair correlation function, see Sec. 5.4.2.3 and Eq. (5.45)

$g(r) \quad$ radial distribution function, spherical symmetric part of $g(\mathbf{r})$

$\gamma \quad$ proportionality factor between collision frequency and temperature in the clustered state, defined in Eq. (5.14)

$L \quad$ linear dimension of the system

$L_{\text {box }} \quad$ edge length of the boxes for the box counting algorithm, see Sec. 5.4.2.2

$L_{\mathrm{co}} \quad$ cross-over length from fractal to compact behavior of the asymptotic cluster, see Sec. 5.4.2

$m \quad$ mass of a particle or a cluster of particles

$\bar{m}(t) \quad$ mean cluster mass, defined in Eq. (5.36)

$n \quad=N / V$, particle density

$n_{\mathrm{cl}} \quad$ number of clusters, see Sec. 5.4.1.3

$N \quad$ number of particles

$N_{\text {box }} \quad$ number of boxes of edge length $L_{\text {box }}$ needed to cover the cluster, see Sec. 5.4.2.2

$N_{m}(t)$ cluster mass distribution, i.e. number of clusters with $m$ particles at time $t$; see Sec. 5.4.1.2

$\mathcal{N}_{i} \quad$ the $i$-th of a total of $n_{\mathrm{cl}}$ clusters, used in Sec. 5.3.4

$\mathcal{N}(i) \quad$ partial cluster of the asymptotic cluster, containing all particles that can be reached with $i$ or less neighbor-to-neighbor steps, starting from a random particle, see Sec. 5.4.2.5

$\nu \quad=\frac{2}{3 \sqrt{\pi}} \cdot \gamma \Delta E^{1 / 2}$, see discussion around Eq. (5.15)

$P_{\mathrm{bb}} \quad$ probability for a bond rupture, i.e. probability that the kin. energy is large enough to break a bond

$\phi \quad=\frac{N}{V} \cdot \frac{\pi}{6} d^{3}$, volume fraction

$\mathbf{r}_{j} \quad$ position of particle $j$

$r_{\mathrm{g}} \quad$ radius of gyration of a cluster, see Eq. (5.33)

$\sigma \quad$ scattering cross section for a pair of particles

$t \quad$ time

$\tilde{t} \quad=\nu t+c$, simplifying definition; see discussion around Eq. (5.22)

$t_{0} \quad$ transition time from free cooling to aggregation, see Eqs. (5.7)

$T \quad$ granular temperature, see Sec. 5.3 and Eq. (5.2)

$T_{0} \quad$ initial value of the temperature; for all simulations $T_{0}=45 \Delta E$ 
$\mathbf{v}_{j} \quad$ velocity of particle $j$

$V \quad=L^{3} ;$ volume of the system

$w(\mathbf{v}) \quad$ velocity distribution, see Sec. 5.3.3

$z \quad$ coordination number, i.e. number of neighbors of a particle

$\bar{z} \quad$ average coordination number 


\section{Bibliography}

Alves, S. G. \& Ferreira, Jr., S. C. 2006 Scaling theory for ballistic aggregation. Phys. Rev. E 73, 051401. 82, 104

Arnott, S., Dover, S. D. \& Elliot, A. 1967 Structure of $\beta$-poly-L-alanine: Refined Atomic Co-ordinates for an Anti-parallel Beta-pleated sheet. J. Mol. Biol. 30, 201. 69, 72, 76, 79

Balanda, K. P. \& MacGillivray, H. L. 1988 Kurtosis: A Critical Review. The American Statistician 42, 111-119. 97

Baldassarri, A., Marconi, U. M. B. \& Puglisi, A. 2002 Influence of correlations on the velocity statistics of scalar granular gases. Europhys. Lett. 58, 14. 83

Ball, R. C. \& Edwards, S. F. 1980 Elasticity and stability of a dense gel. Macromolecules 13 (3), 748-761. 4

Baxter, R. J. 1968 Percus-Yevick Equation for Hard Spheres with Surface Adhesion. J. Chem. Phys. 49, 2770. 98

van Beek, J., Hess, S., Vollrath, F. \& Meier, B. 2002 The molecular structure of spider dragline silk: Folding and orientation of the protein backbone. Proceedings of the National Academy of Sciences of the United States of America (PNAS) 99, 10266-10271. 58

Ben-Naim, E., Chen, S. Y., Doolen, G. D. \& Redner, S. 1999 Shocklike dynamics of inelastic gases. Phys. Rev. Lett. 83, 4069. 83

Ben-Naim, E., Machta, B. \& Machta, J. 2005 Power-law velocity distributions in granular gases. Phys. Rev. E 72, 021302. 95

Ben-Naim, E. \& Machta, J. 2005 Stationary states and energy cascades in inelastic gases. Phys. Rev. Lett. 94, 138001. 95

Blum, J., Wurm, G., Kempf, S. et al. 2000 Growth and Form of Planetary Seedlings: Results from a Microgravity Aggregation Experiment. Phys. Rev. Lett. 85 (12), 2426-2429. 82

Boon, J. P. \& YIP, S. 1991 Molecular Hydrodynamics. Dover Publications, Inc., New York. 114

Botet, R. \& Jullien, R. 1984 Size distribution of clusters in irreversible kinetic aggregation. J. Phys. A 17, 2517-2530. 105

Brey, J. J., Cubero, D. \& Ruiz-Montero, M. J. 1999 High energy tail in the velocity distribution of a granular gas. Phys. Rev. E 59, 1256. 95 
Bridges, F. G., Hatzes, A. \& Lin, D. N. C. 1984 Structure, stability and evolution of saturn's rings. Nature $\mathbf{3 0 9}, 333.82$

Brilliantov, N. V. \& Pöschel, T. 2004 Kinetic Theory of Granular Gases. Oxford University Press, Oxford. 81, 82, 84, 87, 92, 107

Brilliantov, N. V., Pöschel, T., Kranz, W. T. \& Zippelius, A. 2007 Translations and rotations are correlated in granular gases. Phys. Rev. Lett. 98, 128001. 83,124

Broderix, K., Weigt, M. \& Zippelius, A. 2002 Towards finite-dimensional gelation. The European Physical Journal B - Condensed Matter and Complex Systems 29 (3), 441-455. 4, 6, 7, 16, 25, 49, 52

Brookes, M. 2009 The matrix reference manual. Web Access: http://www.ee.ic.ac.uk/hp/staff/dmb/matrix/intro.html. 143

De Bruijn, N. G. 1958 Asymptotic Methods in Analysis, Bibliotheca Mathematica, vol. 4. North-Holland Publishing Co., Amsterdam. 183

Carnahan, N. F. \& Starling, K. E. 1969 Equation of state for nonattracting rigid spheres. J. Chem. Phys. 51, 635. 87

Carnevale, G. F., Pomeau, Y. \& Young, W. R. 1990 Statistics of ballistic agglomeration. Phys. Rev. Lett. 64, 2913-2916. 82, 106

Castillo, H. E. \& Goldbart, P. M. 1998 Elasticity near the vulcanization transition. Phys. Rev. E 58, R24. 28

Castillo, H. E., Goldbart, P. M. \& Zippelius, A. 1994 Distribution of localisation lengths in randomly crosslinked macromolecular networks. EPL (Europhysics Letters) $\mathbf{2 8}$ (7), 519-524. 16, 25, 45, 49, 54

Chissom, B. S. 1970 Interpretation of the kurtosis statistic. The American Statistician 24 (4), 19-22. 97

Corless, R. M., Gonnet, G. H., Hare, D. E. G., Jeffrey, D. J. \& Knuth, D. E. 1996 On the Lambert $W$ function. Advances in Computational Mathmatics 5, 329-359. 27

Dawson, K., Foffi, G., Fuchs, M., Götze, W., Sciortino, F., Sperl, M., Tartaglia, P., Voigtmann, T. \& Zaccarelli, E. 2000 Higher-order glasstransition singularities in colloidal systems with attractive interactions. Phys. Rev. E 63, 011401. 114

Deam, R. T. \& Edwards, S. F. 1976 The theory of rubber elasticity. Philos. Trans. R. Soc. London, Ser. A 280 (1296), 317-353. 4, 6

van Dongen, P. G. J. \& ERnst, M. H. 1985 Dynamic scaling in the kinetics of clustering. Phys. Rev. Lett. 54, 1396. 82, 105 
DurAn, J. 2000 Sands, powders and grains: An introduction to the physics of granular media. Springer, New York. 82

Ennis, B. J., Tardos, G. \& Pfeiffer, R. 1991 A micro-level-based characterization of granulation phenomena. Powder Technology 65, 257. 83

ERDős, P. \& RÉnYI, A. 1960 On the evolution of random graphs. Publ. Math. Inst. Hung. Acad. Sci. 5, 17-61. 25

ERDős, P. \& RÉNYI, A. 1961 On the evolution of random graphs. Bull. Inst. Internat. Statist. 38, 343-347. 25

Esipov, S. E. \& Pöschel, T. 1997 The granular phase diagram. Journal of Statistical Physics 86, 1385. 95

FAmily, F. \& ViCSEK, T. 1985 Scaling of the active zone in the eden process on percolation networks and the ballistic deposition model. J. Phys. A: Math. Gen. 18, L75-L81. 106

Fingerle, A. \& Herminghaus, S. 2006 Unclustering transition in freely cooling wet granular matter. Phys. Rev. Lett. 97, 078001. 83

Fingerle, A. \& Herminghaus, S. 2008 Equation of state of wet granular matter. Phys. Rev. E 77, 011306. 83

Fingerle, A., Röller, K., Huang, K. \& Herminghaus, S. 2008 Phase transitions far from equilibrium in wet granular matter. New J. Phys. 10, 053020. 83, $85,86,123$

Foo, A. W. P., Bini, E., Huang, J., Lee, S. \& Kaplan, D. 2006 Solution behavior of synthetic silk peptides and modified recombinant silk proteins. Applied Physics A 82, 193-203. 58

Fossey, S. \& Kaplan, D. 1999 Polymer Data Handbook, chap. Silk Protein. Oxford University Press. 57

Garzo, V. \& Dufty, J. 1999 Homogeneous cooling state for a granular mixture. Phys. Rev. E 60, 5706. 83

De Gennes, P. G. 1982 Mechanical properties of nematic polymers. In Polymer Liquid Crystals (ed. A. Ciferri, W. R. Kringbaum \& R. B. Meyer), chap. 5. Academic Press, New York. 39

De Gennes, P. G. 1999 Granular matter: a tentative view. Rev. Mod. Phys. 71, S374-S382. 82

Gimel, J. C., Durand, D. \& Nicolai, T. 1995 Transition between flocculation and percolation of a diffusion-limited cluster-cluster aggregation process using three-dimensional monte carlo simulation. Phys. Rev. B 51, 11348. 102 
GLIŠOvić, A. \& SAlditT, T. 2007 Temperature dependent structure of spider silk by X-ray diffraction. Applied Physics A 87, 63-69. 74

Glišović, A., Vehoff, T., Davies, R. J. \& Salditt, T. 2008 Strain dependent structural changes of spider dragline silk. Macromolecules 41, 390-398. 59, 74

Goldbart, P. M., Castillo, H. E. \& Zippelius, A. 1996 Randomly crosslinked macromolecular systems: Vulcanization transition to and properties of the amorphous solid state. Advances in Physics 45 (5), 393. 4, 7, 23, 25, 49, 52, 164

Goldbart, P. M., Mukhopadhyay, S. \& Zippelius, A. 2004 Goldstone-type fluctuations and their implications for the amorphous solid state. Physical Review B $\mathbf{7 0}$ (18), 184201. 4, 14, 17, 18, 26, 28

Goldenfeld, N. 1992 Lectures on Phase Transitions and the Renormalization Group. Frontiers in Physics 85. Westview Press, Boulder, Colorado. 13, 15

Goldhirsch, I., Tan, M.-L. \& Zanetti, G. 1993 A molecular dynamical study of granular fluids I: The unforced granular gas in two dimensions. Journal of Scientific Computing 8, 1. 83, 95

Goldhirsch, I. \& Zanetti, G. 1993 Clustering instability in dissipative gases. Phys. Rev. Lett. 70, 1619. 83

Goldshtein, A. \& Shapiro, M. 1995 Mechanics of collisional motion of granular materials. Part 1. General hydrodynamic equations. J. Fluid Mech. 282, 75. 83

Goldstone, J., Salam, A. \& Weinberg, S. 1962 Broken symmetries. Phys. Rev. 127, 965. 15

Gosline, J., Guerette, P., Ortlepp, C. \& Savage, K. 1999 The mechanical design of spider silks: From fibroin sequence to mechanical function. The Journal of Experimental Biology 202, 3295-3303. 58

Grassberger, P. 1983 On the fractal dimension of the henon attractor. Physics Letters A 97, 224-226. 110, 116

GrubB, D. \& Jelinski, L. 1997 Fiber morphology of spider silk: The effects of tensile deformation. Macromolecules 30, 2860-2867. 57, 58, 69

Haff, P. K. 1983 Grain flow as a fluid-mechanical phenomenon. J. Fluid Mech. 134, 401. 87

Hansen, J.-P. \& McDonald, I. R. 1986 Theory of Simple Liquids, 2nd edn. Academic Press, Inc., San Diego. 114

Hentschel, H. G. E. \& Procaccia, I. 1983 The infinite number of generalized dimensions of fractals and strange attractors. Physica D: Nonlinear Phenomena 8, 435-444. 110 
Herminghaus, S. 2005 Dynamics of wet granular matter. Advances in Physics 54, 221-261. 82,83

Huang, N., Ovarlez, G., Bertrand, F., Rodts, S., Coussot, P. \& Bonn, D. 2005 Flow of wet granular materials. Phys. Rev. Lett. 94, 028301. 83

Huang, W., Baker, G. L. \& Bruening, M. L. 2001 Controlled synthesis of cross-linked ultrathin polymer films by using surface-initiated atom transfer radical polymerization. Angewandte Chemie International Edition 40, 1510. 32

Huemmerich, D., Scheibel, T., Vollrath, F., Cohen, S., Gat, U. \& Ittah, S. 2004 Novel assembly properties of recombinant spider dragline silk proteins. Current Biology 14, 2070-2074. 58

Huemmerich, D., Slotta, U. \& Scheibel, T. 2006 Processing and modification of films made from recombinant spider silk proteins. Applied Physics A 82, 219222.58

Huthmann, M., Orza, J. A. G. \& Brito, R. 2000 Dynamics of deviations from the gaussian state in a freely cooling homogeneous system of smooth inelastic particles. Granular Matter 2, 189. 95

Huthmann, M. \& Zippelius, A. 1997 Dynamics of inelastically colliding rough spheres: Relaxation of translational and rotational energy. Phys. Rev. E 56, R6275. 83

Iijima, M., Nagasaki, Y., Okada, T., Kato, M. \& Kataoka, K. 1999 Corepolymerized reactive micelles from heterotelechelic amphiphilic block copolymers. Macromolecules 32, 1140. 32

Ionov, L., Stamm, M., Minko, S., Hoffmann, F. \& Wolff, T. 2004 Switching and structuring of binary reactive polymer brush layers. Macromolecular Symposia 210, 229. 32

IsRaelachVili, J. 1992 Intermolecular and Surface Forces. Acad. Press, San Diego. 83

Jaeger, H. M., NAgel, S. R. \& Behringer, R. B. 1996 Granular solids, liquids, and gases. Rev. Mod. Phys. 68, 1259. 82

Jiang, Y. \& Leyvraz, F. 1993 Scaling theory for ballistic aggregation. J. Phys. A 26, L179. 82, 105

JiAng, Y. \& LeYvraz, F. 1994 Kinetic properties of ballistic aggregation. Phys. Rev. E 50, 2148-2155. 82, 105, 106

Jullien, R. \& Botet, R. (ed.) 1987 Aggregation and Fractal Aggregates. World Scientific. 101 
Jullien, R. \& KolB, M. 1984 Hierarchical model for chemically limited clustercluster aggregation. J. Phys. A 17, L639. 82, 104

Kamien, R. D., Le Doussal, P. \& Nelson, D. R. 1992 Theory of directed polymers. Physical Review A 45, 8727. 31, 32, 38

Kaplan, D., Adams, W., Farmer, B. \& Viney, C. (ed.) 1994 Silk Polymers: Materials Science and Biotechnology, ACS Symposium Series 544, American Chemical Society, Washington, DC. 58

Kirkpatrick, P. \& BAez, A. V. 1948 Formation of optical images by x-rays. J. Opt. Soc. Am. 38, 766-774. 73

Knuth, D. E. 1973 The Art of Computer Programming, 2nd edn. Addison-Wesley, Reading, Massachusetts. 153

Kolb, M. \& Herrmann, H. 1985 The sol-gel transition modelled by irreversible aggregation of clusters. J. Phys. A 18, L435. 102

Krapivsky, P. L. \& Ben-Naim, E. 2002 Nontrivial velocity distributions in inelastic gases. J. Phys. A: Math. Gen. 35, L147. 83

Kudrolli, A. 2004 Size separation in vibrated granular matter. Rep. Prog. Phys. 67, 209. 82

Landau, H. G. 1952 On some problems of random nets. Bulletin of Mathematical Biology 14, 203-212. 25

Landau, L. D. \& Lifshitz, E. M. 1965 Course of Theoretical Physics, vol. VII: Theory of Elasticity. Pergamon Press, Oxford. 24

Landau, L. D. \& Lifshitz, E. M. 1976 Course of Theoretical Physics, vol. I: Mechanics. Pergamon Press, Oxford. 122

Lian, G., Thornton, C. \& Adams, M. J. 1998 Discrete particle simulation of agglomerate impact coalescence. Chem. Eng. Sci. 53, 3381. 83

Liang, S. \& Kadanoff, L. P. 1985 Scaling in a ballistic aggregation model. Phys. Rev. A 31, 2628. 82

Losert, W., Cooper, D. G. W., Delour, J., Kudrolit, A. \& Gollub, J. P. 1999 Velocity statistics in excited granular media. Chaos 9, 682. 83

Loveless, D. M., Abu-Lail, N. I., Kaholek, M., Zauscher, S. \& Craig, S. L. 2006 Reversibly cross-linked surface-grafted polymer brushes. Angewandte Chemie International Edition 45, 7812. 32

Mao, X., Goldbart, P. M., Xing, X. \& Zippelius, A. 2007 Elastic heterogeneity of soft random solids. Europhysics Letters 80, 26004. 6 
Marchetti, M. C. \& Nelson, D. R. 1993 Translational correlations in the vortex array at the surface of a type-ii superconductor. Physical Review B 47, 1221412223. 32

Marko, J. F. \& Siggia, E. D. 1995 Stretching DNA. Macromolecules 28, 87598770. 38

Marsh, R., Corey, R. \& Pauling, L. 1955a An investigation of the structure of silk fibroin. Biochimica et Biophysica Acta 16, 1-33. 59, 76

Marsh, R., Corey, R. \& Pauling, L. $1955 b$ The structure of tussah silk fibroin. Acta Cryst. 8, 710-715. 74, 75, 76, 77

McNamara, S. 1993 Hydrodynamic modes of a uniform granular medium. Phys. Fluids A 5, 3056. 83

Meakin, P. 1991 Fractal aggregates in geophysics. Reviews of Geophysics 29, 317354. 104, 105

Meling, M. 2006 Modellierung und Strukturanalyse von $\beta$-Faltblattkristalliten in Spinnenseide. Diploma Thesis, Göttingen University. 69

Mukhopadhyay, S., Goldbart, P. M. \& Zippelius, A. 2004 Goldstone fluctuations in the amorphous solid state. Europhysics Letters 67 (1), 49-55. 4, 14, 17, $18,26,28$

Nelson, D. R. 2002 Defects and Geometry in Condensed Matter Physics, chap. 9. Cambridge University Press, Cambridge, UK. 39

Nie, X., Ben-Naim, E. \& Chen, S. Y. 2002 Dynamics of freely cooling granular gases. Phys. Rev. Lett. 89, 204301. 83

VAn Noije, T. \& ERnst, M. 1998 Velocity distributions in homogeneous granular fluids: the free and the heated case. Granular Matter 1, 57-64. 95

Panyukov, S. \& Rabin, Y. 1996 Statistical physics of polymer gels. Physics Reports 269, 1-131. 4

Peng, W., Castillo, H. E., Goldbart, P. M. \& Zippelius, A. 1998 Universality and its origins at the amorphous solidification transition. Phys. Rev. B 57, 839. $14,26,49$

Peng, W. \& Goldbart, P. M. 2000 Renormalization-group approach to the vulcanization transition. Physical Review E 61 (4), 3339. Copyright (C) 2009 The American Physical Society; Please report any problems to prola@aps.org. 28

Puxkandl, R., Zizak, I., Paris, O., Keckes, J., Tesch, W., Bernstorff, S., Purslow, P. \& Fratzl, P. 2002 Viscoelastic properties of collagen: synchrotron radiation investigations and structural model. Philosophical Transactions of the Royal Society of London, Series B 357, 191-197. 58 
Ramakrishnan, C. \& Ramachandran, G. N. 1965 Stereochemical Criteria for Polypeptide and Protein Chain Conformations - II. Allowed Conformations for a Pair of Peptide Units. Biophys J. 5, 909-933. 70

Rammensee, S., Huemmerich, D., Hermanson, K., Scheibel, T. \& Bausch, A. 2006 Rheological characterization of hydrogels formed by recombinantly produced spider silk. Applied Physics A 82, 261-264. 58

Riekel, C., Bränden, C., Craigc, C., Ferreroa, C., Heidelbacha, F. \& MÜLLER, M. $1999 a$ Aspects of x-ray diffraction on single spider fibers. International Journal of Biological Macromolecules 24, 187-195. 58

Riekel, C. \& DAvies, R. J. 2005 Applications of synchrotron radiation microfocus techniques to the study of polymer and biopolymer fibers. Current Opinion in Colloid $\mathcal{E}$ Interface Science 9, 396-403. 73

Riekel, C., Madsen, B., Knight, B. \& Vollrath, F. 2000 X-ray diffraction on spider silk during controlled extrusion under a synchrotron radiation $\mathrm{x}$-ray beam. Biomacromolecules 1, 622-626. 58

Riekel, C., Mueller, M. \& Vollrath, F. $1999 b$ In situ x-ray diffraction during forced silking of spider silk. Macromolecules 32, 4464-4466. 58

Riekel, C., Rössle, M., Sapede, D. \& Vollrath, F. 2004 Influence of $\mathrm{co}_{2}$ on the micro-structural properties of spider dragline silk: X-ray microdiffraction results. Naturwissenschaften 91, 30-33. 58

Riekel, C. \& Vollrath, F. 2001 Spider silk fibre extrusion: Combined wideand small-angle x-ray microdiffraction experiments. International Journal of Biological Macromolecules 29, 203-210. 58

Roschger, P., Grabner, B., Rinnerthaler, S., Tesch, W., Kneissel, M., Berzlanovich, A., Klaushofer, K. \& Fratzl, P. 2001 Structural development of the mineralized tissue in the human 14 vertebral body. Journal of Structural Biology 136, 126-136. 58

Rottereau, M., Gimel, J., Nicolai, T. \& Durand, D. 2004 "monte carlo simulation of particle aggregation and gelation: Ii. pair correlation function and structure factor". Eur. Phys. J. E 15, 141. 116

Sapede, D., Seydel, T., Forsyth, V., Koza, M., Schweins, R., Vollrath, F. \& Riekel, C. 2005 Nanofibrillar structure and molecular mobility in spider dragline silk. Macromolecules 38, 8447-8453. 59

Scheibel, T. 2004 Spider silks: recombinant synthesis, assembly, spinning and engineering of synthetic proteins. Microbial Cell Factories 3, 14. 58

Solomonoff, R. \& Rapoport, A. 1951 Connectivity of random nets. Bulletin of Mathematical Biology 13, 107-117. 25 
Stanley, H. E. \& Ostrowsky, N. (ed.) 1986 On Growth and Form: Fractal and Non-Fractal Patterns in Physics. NATO ASI Series E 100. Martinus Nijhoff Publishers. 104

Stegun, I. A. \& Abramowitz, M. 1954 Handbook of Mathematical Functions, 9th edn. Dover Publications Inc., New York. 90

Thornton, C., Yin, K. K. \& Adams, M. J. 1996 Numerical simulation of the impact fracture and fragmentation of agglomerates. J. Phys. D 29, 424. 83

Trizac, E. \& Hansen, J.-P. 1995 Dynamic scaling behaviour of ballistic coalescence. Phys. Rev. Lett. 74, 4114. 82, 105

Trizac, E. \& Krapivsky, P. L. 2003 Correlationes in ballistic processes. Phys. Rev. Lett. 91, 218302. 82, 106, 107

Ulrich, S., Aspelmeier, T., Roeller, K., Fingerle, A., Herminghaus, S. \& Zippelius, A. 2009a Cooling and aggregation in wet granulates. Phys. Rev. Lett. 102, 148002. 83

Ulrich, S., Aspelmeier, T., Zippelius, A., Roeller, K., Fingerle, A. \& Herminghaus, S. $2009 b$ Dilute wet granulates: Nonequilibrium dynamics and structure formation. Phys. Rev. E 80, 031306. 83

Ulrich, S., Glišović, A., Salditt, T. \& Zippelius, A. 2008 Diffraction from the $\beta$-sheet crystallites in spider silk. The European Physical Journal E 27, 229. 60

Ulrich, S., MaO, X., Goldbart, P. M. \& Zippelius, A. 2006 Elasticity of highly cross-linked random networks. Europhys. Lett. 76 (4), 677. 18

Ulrich, S., Zippelius, A. \& Benetatos, P. 2010 Random networks of crosslinked directed polymers. Phys. Rev. E 81, 021802. 56

Umbanhowar, P. B., Melo, F. \& Swinney, H. L. 1996 Localized excitations in a vertically vibrated granular layer. Nature 382, 793. 82

ViCSEK, T. \& FAmily, F. 1984 Dynamic scaling for aggregation of clusters. Phys. Rev. Lett. 52, 1669-1672. 105

Vollrath, F. \& Porter, D. 2006 Spider silk as a model biomaterial. Applied Physics A 82, 205-212. 58

Warwicker, J. O. 1954 The Crystal Structure of Silk Fibroin. Acta Cryst. 7, 565-573. 76

Warwicker, J. O. 1960 Comparative Studies of Fibroins - II. The Crystal Structures of Various Fibroins. J. Mol. Biol. 2, 350-362. 58, 59, 60, 70 
Westbrook, C. D., Ball, R. C., Field, P. R. \& Heymsfield, A. J. 2004 Theory of growth by differential sedimentation, with application to snowflake formation. Phys. Rev. E 70, 021403. 82, 104

Willett, C. D., Adams, M. J., Johnson, S. A. \& Seville, J. P. K. 2000 Capillary bridges between two spherical bodies. Langmuir 16, 9396-9405. 82

Willis, B. T. M. \& Pryor, A. W. 1975 Thermal Vibrations in Crystallography, 1st edn., chap. 4. Cambridge University Press. 68

Xu, P., Tang, H., Li, S., Ren, J., Kirk, E. V., Murdoch, W. J., Radosz, M. \& Shen, Y. 2004 Enhanced stability of core-surface cross-linked micelles fabricated from amphiphilic brush copolymers. Biomacromolecules 5, 1736. 32

Zaburdaev, V. Y., Brinkmann, M. \& Herminghaus, S. 2006 Free cooling of the one-dimensional wet granular gas. Phys. Rev. Lett. 97, 018001. 83, 95

Zallen, R. 1983 The Physics of Amorphous Solids. John Wiley \& Sons Inc. 4

Zbilut, J., Scheibel, T., Huemmerich, D., Webber, C., Colafranceschi, M. \& Guiliani, A. 2006 Statistical approaches for investigating silk properties. Applied Physics A 82, 243-251. 58

Zubay, G. 1998 Biochemistry, 4th edn. McGraw-Hill Education. 60, 61 


\section{Index}

alanine, 58, 69, 70, 76

alignment interaction, 37

amorphous matrix, 58

amphiphilic block copolymers, 32

angular distribution function

for crystallites, 64

angular momentum, 99

Ansatz for the order parameter, 18-20

antiparallel beta-sheet, 70, 75

asymptotic cluster

coordination number, 118-120

fractal dimension

box counting method, 110-112

correlation dimension, 116

from radius of gyration, 108

pair correlation function, 114-117

asymptotic limit of cooling, see

free cooling, late stage

average coordination number, 25

for the RLP model, 7

ballistic aggregation models, 106

beta-sheets, 58, 60, 70

classification of Warwicker, 59

Bombyx mori, 76

bond-breaking

distance $d_{\mathrm{c}}, 82,84,86,118$

energy $\Delta E, 82-84$

probability $P_{\mathrm{bb}}, 86,90,97$

boundary effects

for cross-linked directed chains, 5254

bridge formation, 83

capillary bridge, see liquid bridge

chain density, see mean chain density

classical theory of rubber elasticity, 28

cluster (of granular particles)

definition, 98 number of $\sim \mathrm{s}, 106$

partial $\sim, 108,122$

cluster mass distribution $N_{m}(t), 102,105$

scaling, 105-106

clustering instability, 95

coefficient of restitution, 82,84

effective $\sim$ for wet granulates, 84

coherent scattering, 67-68, 187

collision frequency, 86, 87, 91, 107

continuous background, 64-65, 187

continuous symmetry, 14, 15

cooling, see free cooling

coordination number, see also cross-link density

for the RLP model, 7, 25

for wet granulates, 118-120

correlation dimension, 116

correlations between crystallites, 67-68, 187

cross-link

configuration, 4, 6, 36

density, 24, 25, 150, 180

fluctuations, 25

for directed polymers, 36-37

Hamiltonian, 5, 37

in spider silk, 58, see crystallites

cross-linking, 7,8

crystallites, 58, 60

addition of glycine, 76

ensemble, 63-64

modeling, 60-61

tilting of $\sim, 63$

variations between $\sim, 72,76$

Deam-Edwards distribution, 6-8, 39

deformation field $u_{\perp}$

expansion in, 140

introduction, 19 
delocalized particles, 16, 18, 26, 45

density fluctuations, 12

diffusion limited cluster-cluster aggregation, 103

dimensionless inverse and squared localization length, 50

dimensionless inverse temperature, 91

dimensionless system height, 48

disorder

in beta-sheets, 76

in replica calculations, see cross-link configuration

disorder average, 6-7, 39-41

ed free energy, 7, 23-24, 40

$\sim$ ed replicated dentity, 10

distribution function

for crystallite orientations, 64

for crystallite positions, 63

distribution of localization length, 19

dragline fibers, 57

ensemble of crystallites, 63-64

Euler angles, 63

illustration, 64

excluded-volume interaction

for directed polymers, 37, 43

for the RLP model, 5

infinitely strong limit, 13, 44

fiber axis, 63

field theory

introduction, $12-13$

flocculation regime, 102

flux lines, 32

form factor, 60

effective $\sim, 66$

formation of a liquid bridge, 83

fractal dimension $D_{\mathrm{f}}$

box counting method, 110

time evolution, 120

correlation dimension, 116

from radius of gyration, 104, 108

free cooling

early stage, $87-90$ late stage, $90-93$

free energy, 7, 40

gel fraction, 16, 18, 25-27, 45

gel phase, 4, 9, 14, 17

gelation transition, 1, 4, 14, 26, 42, 49, 55

glycine (substitution inside a crystallite), 76

Goldstone fluctuations, $14-15$

Ansatz with, 18-20

Goldstone modes, 15

granular temperature, 86

Hausdorff dimension, 110

high energy tails, 95

higher replica sector, 11, 42, 136, 151, 164

Hubbard-Stratonovich transformation, $12,43,130,164$

incoherent scattering, 66-67

incompressible deformations, see shear deformations

inertia tensor, see moment of inertia tensor

infinite cluster, see percolating cluster

internal temperature, 99-101

kurtosis, 97

Laplace transformation, 175

leptokurtic distribution, 97

linearization, see Hubbard-Stratonovich transformation

liquid bridge, 82

formation, 83

liquid film

on granular particles, 82

liquid phase, see sol phase

localization length, 10, 16, 45

distribution of, 16, 19, 46

illustration, 10, 18

normalized distribution of, 50

final result, 52 
normalized inverse and squared, 50 typical, $54-55$

localized particles, 9, 26, 42, 45

logarithmic time decay, 92

macroscopic cluster, see percolating cluster

mean chain density, 42, 164

mean cluster mass, 105, 106

scaling, 107

mean particle density

for the RLP model, 11, 130

for the wet granular system, 87

moment of inertia tensor, 99, 122

neighborhood definition, 98

nematic field

polymers in a $\sim, 38-39$

nematic polymers, 31

normalization of length scales, 49

normalized inverse and squared localization length, 50

number of clusters, 106

oblate object, 122

one replica sector, 11, 42, 154

one-particle partition function $\mathfrak{z}$

of cross-linked directed chains introduction, 43, 167

with additional interactions, 160

of the RLP model

expansion, 137

introduction, 13, 132

order parameter $\Omega$

for directed polymers

Ansatz, 45-47

for the RLP model

Ansatz, 15-20

in the 1RS, 154

insertion into $f_{n+1}\{\Omega\}, 145$

insertion into $\mathfrak{z}, 137$

meaning, 132

pair correlation function, 114

at contact, 87 parallel beta-sheet, 70,75

partial cluster, 108, 122

particle density, see mean particle density

partition function $Z_{\mathcal{C}}$

of cross-linked directed polymers, 39

of the RLP model, 6

percolating cluster, 4, 9, 16, 42, 101, 102

percolation theory, 1, 102

percolation time, 102

periodic boundary conditions, 86

permanent cross-links, see cross-link

physical deformation field, 21

platykurtic distribution, 97

poly-L-alanine, 58, 76, 79

polyalanine, see alanine or poly-L-alanine

polymer brushes, 31

polymers in a nematic field, 38-39

primitive vectors, 60

principal axes, 122

principal moments of inertia, 122

principal vectors, 70

prolate object, 122

pure shear deformations, see shear deformations

quenched disorder, see cross-link configuration

radial distribution function, 114

Percus-Yevick solution, 114, 115

radius of gyration, 103, 108

of a single polymer chain, 34,48

randomly cross-linked particle model, 3

replica free energy $f_{n+1}\{\Omega\}$

of cross-linked directed chains introduction, 43, 167

saddle point equation, 44-45

of the RLP model

introduction, 13, 132

result, 22, 149

saddle point value, 22

replica free energy $\tilde{f}_{n+1}\left\{\hat{r}_{j}\right\}, 8,41$ 
replica partition function $\mathcal{Z}_{n+1}$ for cross-linked directed chains, 41 for the RLP model, 8

replica trick, 7, 40

replicated density field introduction

for directed polymers, 41 for the RLP model, 9-11

replicated vectors $\hat{r}$

introduction, 8

longitudinal component $\mathbf{r}_{\|}, 17$

properties, 17, 125-126

transverse component $r_{\perp}, 17$

restitution coefficient, 84

rotational temperature, 99-101

saddle point equation, 44-45

saddle point solution

family of $\sim \mathrm{s}, 17-18$

fluctuations around the $\sim, 135$

for the RLP model, 16-18

scattering amplitude

of a single crystallite $A(\mathbf{q}), 67-69$

peak shift, 69

scattering cross section, 87,89

scattering function $G(\mathbf{q}), 60,65$

effect of the parameters, 74

experimental, 73

from the model, $75-78$, see also

Eqs. (4.11), (4.20)

peak shift, 69

side maxima, 76,78

shear deformations, 18-20

physically relevant $\sim, 20$

shear modulus $G, 22,24,27-29,152$

single fiber diffraction, 58, 73-74

single polymer chain

clamped in space, 34-36

radius of gyration, 33-34

sol phase, 4, 9, 14

sol-gel transition, see gelation transition spontaneous symmetry breaking, 15, 17- statistical parameters of the spider silk model, 74-75

structural parameters of the spider silk model, 74-75

system spanning cluster, see percolating cluster

tenacy, 58

tensile strength, 57

thick film model, 85

thin film model, 85

comparison to thick film model, 8890, 119-120

time scale

for free cooling $t_{0}, 87$

translational invariance, 17

translational temperature, 99-101

tussah silk, 76

type-II superconductors, 32

unit cell

atomic configuration, 69-72

shifts, 72

unshifted, 69

variations between $\sim \mathrm{s}, 72,76$

universality

of the RLP model, 13

velocity distribution $w(\mathbf{v}), 90,95-98$

volume fraction, 86

WAXS (wide angle X-ray scattering), 73 worm-like chain, 38

XY-model, 15

zero replica sector, 12, 164 


\title{
Curriculum Vitae
}

\author{
Stephan Ulrich \\ Hannoversche Str. 137 \\ 37077 Göttingen
}

Web: http://wwwuser.gwdg.de/ sulrich/research

\section{Bibliographische Daten}

\author{
Geburtsdatum 16. September 1980 \\ Geburtsort Karlsruhe \\ Staatsangehörigkeit deutsch
}

\section{Wissenschaftlicher Bildungsgang}

$\begin{array}{ll}2000 & \text { Abitur am Werner-von-Siemens-Gymnasium Regensburg } \\ 2000-2001 & \begin{array}{l}\text { Fernstudium Physik im FiPS-Programm der } \\ \text { Universität Kaiserslautern }\end{array} \\ 2001-2003 & \begin{array}{l}\text { Physikstudium an der Universität Würzburg } \\ 2002\end{array} \\ \text { Vordiplom } \\ 2003-2004 & \begin{array}{l}\text { Master-Studiengang an der University of Texas at Austin } \\ \text { Arbeit an Segregation in granularer Materie im }\end{array} \\ & \text { Center for Nonlinear Dynamics } \\ \text { Dez. 2004 } & \text { Master Abschluss } \\ \text { seit 2005 } & \text { Promotion an der Georg-August-Universität Göttingen }\end{array}$

\section{Publikationen}

- Random networks of cross-linked directed polymers angenommen von Phys. Rev. E Stephan Ulrich, Annette Zippelius and Panayotis Benetatos

- Dilute Wet Granulates: Nonequilibrium Dynamics and Structure Formation Phys. Rev. E 80, 031306 (2009)

Stephan Ulrich, Timo Aspelmeier, Annette Zippelius, Klaus Roeller, Axel Fingerle and Stephan Herminghaus

- Cooling and aggregation in wet granulates Phys. Rev. Lett. 102, 148002 (2009)

Stephan Ulrich, Timo Aspelmeier, Klaus Roeller, Axel Fingerle, Stephan Herminghaus and Annette Zippelius 
- Diffraction from the $\beta$-sheet crystallites in spider silk Eur. Phys. J. E 27, 229 (2008)

Stephan Ulrich, Anja Glišović, Tim Salditt and Annette Zippelius

- Influence of friction on granular segregation

Phys. Rev. E 76, 042301 (2007)

Stephan Ulrich, Matthias Schröter and Harry L. Swinney

- Granulare Medien: Der Paranuss-Effekt

Physik in unserer Zeit 38, 266 (2007)

Stephan Ulrich and Matthias Schröter

- Elasticity of highly cross-linked random networks

Europhys. Lett. 76, 677 (2006)

Stephan Ulrich, Xiaoming Mao, Paul M. Goldbart and Annette Zippelius

- Mechanisms in the size segregation of a binary granular mixture Phys. Rev. E 74, 011307 (2006)

Matthias Schröter, Stephan Ulrich, Jennifer Kreft, Jack B. Swift and Harry

L. Swinney

Göttingen, den 3. 2. 2010 